\title{
Representações retangulares de grafos planares
}

\author{
Guilherme Puglia Assunção \\ DISSERTAÇÃO APRESENTADA \\ $\mathrm{AO}$ \\ Instituto DE MATEMÁtica e Estatística \\ $\mathrm{DA}$ \\ Universidade De SÃo Paulo \\ PARA \\ OBTENÇÃO DO TÍTULO \\ $\mathrm{DE}$ \\ Mestre \\ Programa: Ciência da Computação \\ Orientador: Prof. Dr. Carlos Eduardo Ferreira
}

Durante o desenvolvimento deste trabalho o autor recebeu auxílio financeiro do CNPq

São Paulo, maio de 2012 


\section{Representações retangulares de grafos planares}

Esta dissertação trata-se da versão original do aluno (Guilherme Puglia Assunção). 


\section{AGRADECIMENTOS}

Primeiramente, agradeço a Deus pela oportunidade de desenvolver este trabalho e de encontrar pessoas nesta jornada que me fazem crescer tanto intelectualmente como moralmente.

Ao Professor Dr. Carlos Eduardo Ferreira que, além de seu papel de orientador, foi um companheiro nos momentos difíceis que passei durante esta etapa da minha vida. Fico sem palavras para demonstrar o quanto sou grato pela sua paciência e, principalmente, pela sua dedicação à minha orientação.

Aos professores do grupo de Otimização Combinatória do Instituto de Matemática e Estatística da Universidade de São Paulo. Fico lisonjeado de ter sido membro deste grupo e muito agradecido pelo conhecimento que obtive convosco. Aos meus amigos de laboratório, de disciplina e da maratona de programação pelos bons momentos que me proporcionaram. A vocês meus amigos, desejo-lhes sucesso na vida profissional e pessoal.

Ao meu amigo Eduardo Henrique Molina da Cruz, exemplo de força, inteligência e dedicação. Espero poder sempre contar com sua amizade que mesmo muito longe sempre esteve presente.

Aos meus amigos Felipe Bernardes, Hélio Mesquita, Renan de Oliveira (vulgo Burca), Thiago Okada e João Lourenço Fadel. A vocês que sempre estão presentes em minhas realizações.

Aos meus amigos de república, Fredy, Alexandre, Bustamante (Bubito) e Paulo Victor Eufrásio (PV). Sou muito, mas muito grato a todos vocês por serem pacientes e fazerem que a nossa casa fosse um ambiente muito agradável. Muito obrigado.

Ainda, a você meu amigo e companheiro PV. Espero que nossa amizade continue após esta etapa. Deseje-lhe muitas felicidades.

Impossível não ser eternamente grato à minha família. Primeiramente, pela compreensão por não estar presente fisicamente na maior dificuldade que já enfrentamos. Saibam que amo muito vocês e este amor me dá forças para superar as adversidades diárias. Mamãe e 
Papai, sou muito grato por todo o apoio que me deram, desde o momento de inscrição até a finalização deste trabalho.

Leozinho, precisava reservar um parágrafo somente para ti. Agradeço-lhe por ter me mostrado coisas que realmente tem valor nesta vida. Sou muito orgulhoso de ser seu irmão. Esta dissertação é dedicada a ti.

Finalmente, agradeço a minha namorada Camila Zanette. Camilinha, você foi a pessoa mais importante durante toda esta etapa. Foi com você que compartilhei minhas angústias, felicidades e realizações. Também lhe agradeço pela sua paciência e compreensão. Meu muitíssimo obrigado. 


\section{RESUMO}

\section{Representações retangulares de grafos planares}

Uma representação retangular de um grafo plano $\mathbb{G}$ é uma representação de $\mathbb{G}$, onde cada vértice é desenhado como um retângulo de modo que dois retângulos devem compartilhar algum segmento de seus lados se e somente se existe uma aresta em $\mathbb{G}$ entre os vértices correspondentes aos retângulos. Ainda, a representação de $\mathbb{G}$ deve formar um retângulo e não deve existir buracos, ou seja, toda região interna deve corresponder a algum vértice de $\mathbb{G}$.

Um desenho retangular de um grafo plano $\mathbb{H}$ é um desenho de $\mathbb{H}$, onde todas as arestas são desenhadas como segmentos horizontais ou verticais. Ainda, todas as faces internas são retângulos e as arestas que incidem na face externa também formam um retângulo.

Nesta dissertação, apresentamos os principais trabalhos existentes na literatura para problemas associados à representação retangular. Também apresentamos resultados para problemas associados ao desenho retangular. Por fim, apresentamos o algoritmo que desenvolvemos para determinar as coordenadas dos vértices de um desenho retangular quando a orientação das arestas já foram determinadas.

Palavras-chave: Otimização combinatória, desenho de grafos, teoria dos grafos, planaridade, representação retangular, desenho retangular. 



\section{ABSTRACT}

\section{Rectangular representations of plane graphs}

A rectangular representation of a plane graph $\mathbb{G}$ is a representation of $\mathbb{G}$, where each vertex is drawn as a rectangle, such as two rectangles have to share some boundary if and only if exist an edge in $\mathbb{G}$ between the corresponding vertices. Also, the representation of $\mathbb{G}$ must form a rectangle and does not contain any holes, in other words, every point inside the formed rectangle must correspond to some vertex of $\mathbb{G}$.

A rectangular drawing of a plane graph $\mathbb{H}$ is a drawing of $\mathbb{H}$, where all edges are drawn either in vertical or in horizontal. Also, every internal face is a rectangle and the edges which are incident in the external face define a rectangle.

In this dissertation, we present the main studies in the literature for problems associated with the rectangular representation. We also present results for problems associated with rectangular drawing. Finally, we present the algorithm we developed to determine the coordinates of the vertices of a rectangular drawing when the orientation of the edges have been determined.

Keywords: Combinatorial Optimization, graph drawings, graph theory, planarity, rectangular representation, rectangular drawing. 

1 Introdução 1

2 Conceitos e definições básicas $\quad 5$

I Problema da Representação Retangular $\quad 11$

3 Problema da representação retangular $\quad 13$

3.1 Definição do Problema . . . . . . . . . . . . . . . . . . . . 14

3.2 Revisão Bibliográfica . . . . . . . . . . . . . . . . . . . . . 17

4 Caracterização de grafos com representações retangulares $\quad 21$

4.1 Resultado principal . . . . . . . . . . . . . . . . . . 21

4.2 Construção de uma 4-extensão para um bloco . . . . . . . . . . . . . . . . 37

4.3 Construção de uma 4-extensão para grafos gerais . . . . . . . . . . . . . 41

4.4 Algoritmo de Koźmiński e Kinnen . . . . . . . . . . . . . . . . . . . . 43

5 Algoritmo de Bhasker e Shani $\quad 47$

5.1 Grafo dos caminhos dirigidos . . . . . . . . . . . . . . . 47

5.2 Visão geral do algoritmo de Bhasker e Shani . . . . . . . . . . . . . . . . . 50

5.3 Algoritmo para construir um grafo dos caminhos dirigidos . . . . . . . . 54

5.4 Algoritmo de Bhasker e Shani . . . . . . . . . . . . . . . . . . . 65

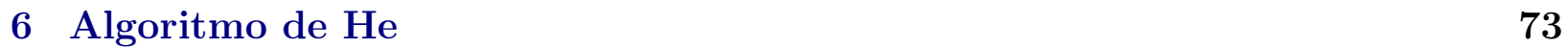


6.1 Rotulação regular das arestas de um grafo plano triangulado . . . . . . . . . 73

6.2 Propriedades das rotulação regular das arestas . . . . . . . . . . . . . . 80

6.3 Numeração consistente de um $s, t$-grafo . . . . . . . . . . . . . . . . . . . 81

6.4 Algoritmo de He baseado em $s, t$-caminhos . . . . . . . . . . . . . . 87

7 Redução do problema de representação retangular $\quad 93$

7.1 Redução do problema de representação retangular sem determinar os cantos 105

7.2 Caracterização de Lai e Leinwand . . . . . . . . . . . . . . . . . . . . . . 106

II Problema do Desenho Retangular

8 Problema do desenho retangular 115

8.1 Revisão Bibliográfica . . . . . . . . . . . . . . . . . . . 117

9 Caracterização de Thomassen 119

9.1 Transformação do grafo da entrada . . . . . . . . . . . . . . . . . . . . 119

9.2 Caracterização de Thomassen . . . . . . . . . . . . . . . . . . . . 121

10 Algoritmo de Rahman et al. para o problema de desenho retangular

10.1 Visão geral sobre o algoritmo . . . . . . . . . . . . . . . . . . . 130

10.2 Conceitos preliminares . . . . . . . . . . . . . . . . 131

10.3 Algoritmo para o problema de desenho retangular . . . . . . . . . . . . . 144

11 Algoritmo para determinar as coordenadas dos vértices 151

11.1 Algoritmo de Rahman et al. para determinar os vértices . . . . . . . . . . . . 152

11.2 Algoritmo para determinar os vértices usando ord. topológica . . . . . . . . . 154

12 Conclusões 
12.2 Sugestões para Pesquisas Futuras . . . . . . . . . . . . . 161

A Implementação do algoritmo para construir um grafo dos caminhos dirigidos

1.1 Variáveis . . . . . . . . . . . . . . . . . . . . 163

1.2 Algoritmo . . . . . . . . . . . . . . . . . . 167

$\begin{array}{lr}\text { Referências Bibliográficas } & 189\end{array}$ 



\section{1 \\ INTRODUÇÃO}

Problemas que envolvem o desenho de grafos vêm sendo estudados há alguns anos e são de grande importância para a área da otimização combinatória, principalmente pelo fato de estarem presentes tanto em problemas teóricos quanto práticos.

O desenvolvimento de algoritmos para os problemas que envolvem desenho de grafos é muito interessante pelo fato de proporcionar resultados teóricos relevantes que envolvem grafos planares. Neste trabalho, nos restringimos a estudar um problema de representação de grafos, mais precisamente ao problema da representação retangular.

Uma representação retangular de um grafo plano $\mathbb{G}$ é uma representação de $\mathbb{G}$, onde cada vértice é desenhado como um retângulo de modo que dois retângulos devem compartilhar algum segmento de seus lados se e somente se existe uma aresta em $\mathbb{G}$ entre os vértices correspondentes aos retângulos. Ainda, a representação de $\mathbb{G}$ deve formar um retângulo e não devem existir buracos, ou seja, toda região interna deve corresponder a algum vértice de $\mathbb{G}$. A figura 1.1 ilustra um grafo $\mathbb{G}$ que é entrada do problema e sua respectiva representação retangular. Na figura 1.2 mostramos uma representação que não é retangular por causa da existência de buracos.

O problema de representação retangular possui aplicações práticas no projeto de circuitos VLSI e na planta baixa de projetos arquitetônicos.

Em um projeto de um circuito VLSI é dado um conjunto de componentes eletrônicos 


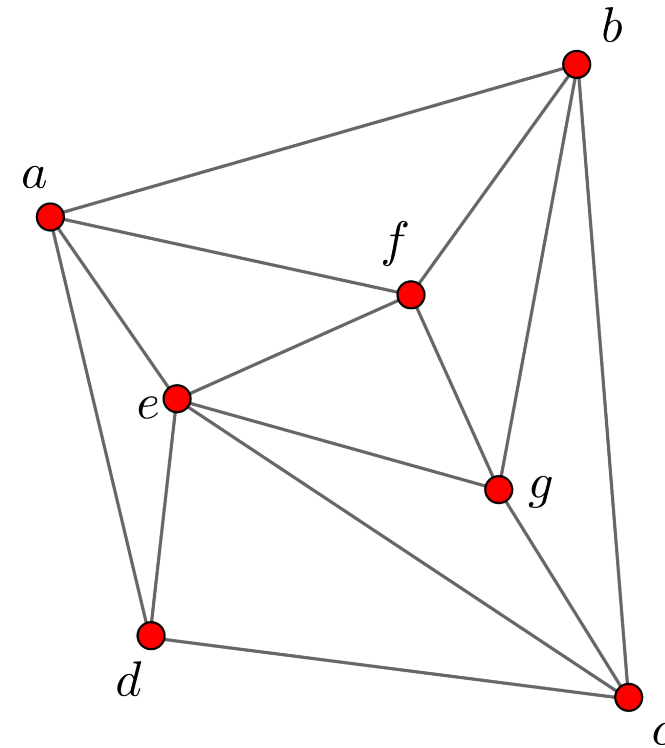

(A)

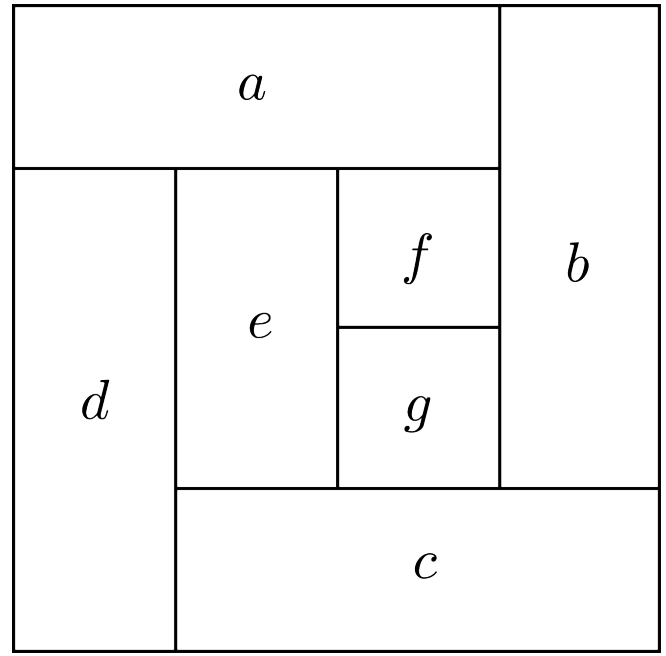

(B)

Figura 1.1: (A) Grafo plano da entrada do problema de representação retangular. Representação retangular de $(A)$.

e quais componentes devem estar diretamente ligados e deseja-se encontrar a posição dos componentes eletrônicos e o desenho das trilhas que devem conectá-los sem que duas trilhas se intersectem. Podemos simplificar este problema se considerarmos que o circuito deve ser retangular, os componentes serão retângulos e dois componentes estão conectados se estes são adjacentes na representação do circuito. Deste modo, podemos modelar as restrições de conexidade como um grafo, onde os vértices correspondem aos componentes e a existência de uma aresta entre dois vértice indica que deve existir uma trilha que os conectem. Note que este grafo que construímos é o grafo de entrada do problema de representação retangular e um circuito válido é uma solução do problema de representação retangular.

Desta forma, o problema da representação retangular pode ajudar a resolver algumas questões acerca do projeto de circuito VLSI. Por exemplo, é natural nos perguntarmos sobre a existência de um circuito que satisfaça as restrições de conexidade, pois como existem muitos componentes envolvidos, nem sempre será possível visualizar tal circuito. Consequentemente, caso seja possível satisfazer as restrições dadas, devemos saber como construir um dos possíveis circuitos.

O projeto de plantas baixas de projetos arquitetônicos possui as mesmas características que a do projeto de circuitos VLSI. Nesta aplicação, serão dados os cômodos das casas e a relação de quais cômodos deverão ser vizinhos. Perceba que existe um grafo equivalente às restrições de adjacências que é entrada do problema de representação retangular. Do mesmo modo que no projeto de circuito VLSI, solucionar o problema de representação retangular auxilia na solução de alguns problemas referentes ao projeto arquitetônico. Note que o problema da viabilidade também existe, mesmo que este possua menos elementos, nem sempre é possível visualizar uma planta baixa que satisfaça as restrições. Entretanto, 


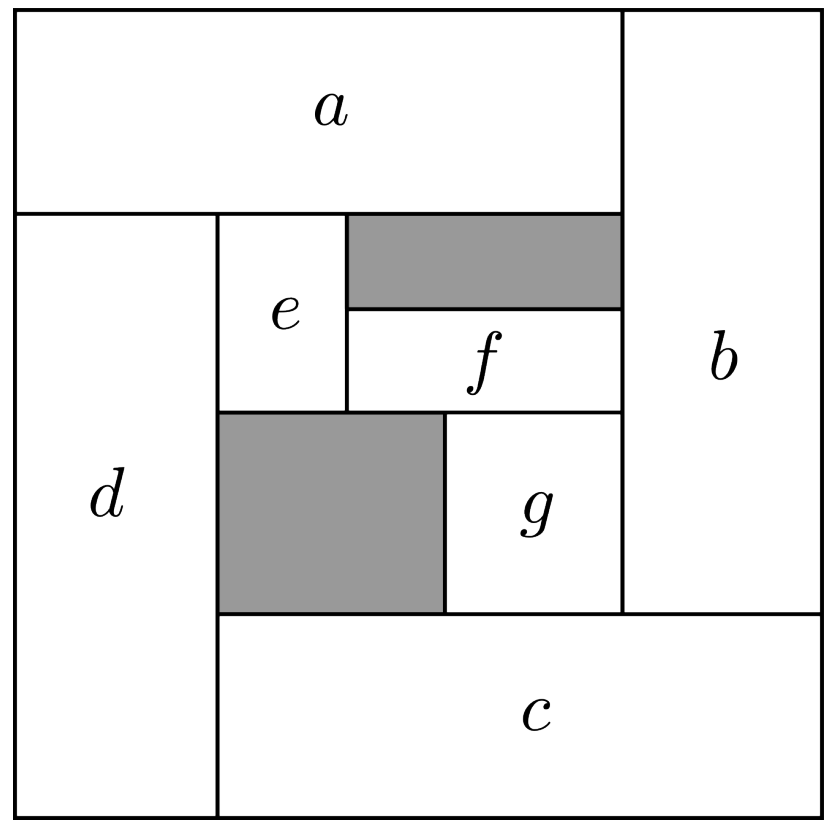

Figura 1.2: Exemplo de uma representação de um grafo plano que não é uma representação retangular.

além de sabermos como construir uma planta baixa, em algumas situações seria conveniente ilustrar todas as plantas baixas possíveis.

A partir destas duas aplicações surge a necessidade de algoritmos eficientes para verificar se é possível construir um representação retangular e caso seja, para saber como construíla. Ainda, motivado pela aplicação no projeto de plantas baixas, seria interessante que encontrássemos um método que consiga construir todas as representações retangulares.

Como mostramos neste trabalho, já existem resultados que implicam em algoritmos eficientes para o problema da representação retangular. Neste contexto, o objetivo deste trabalho é apresentar e discutir os principais resultados existentes na literatura para o problema da representação retangular de grafos planos.

Vamos dividir este trabalho em duas partes, uma para apresentar resultados relacionados ao problema de representação retangular e outra para apresentar resultados relacionados ao problema de desenho retangular. Futuramente, iremos mostrar que os problemas de representação retangular e o de desenho retangular são equivalentes.

No capítulo 2 definimos alguns conceitos básicos e introduzimos a notação que será utilizada durante todo o trabalho. A parte I é composta pelos capítulos 3, 4, 5, 6 e 7 . O capítulo 3 define o problema de representação retangular. No capítulo 4 apresentamos a caracterização do problema de representação retangular. Nos capítulos 5 e 6 apresentamos dois algoritmo lineares para solucionar o problema da representação retangular. No capítulo 7 apresentamos uma redução do problema da representação ao problema de encontrar um emparelhamento perfeito em grafos bipartidos. 
A parte II é composta pelos capítulos 8, 9, 10 e 11. O capítulo 8 define o problema do desenho retangular. O capítulo 9 apresenta uma caracterização para o problema do desenho retangular. Enquanto o capítulo 10 apresenta um algoritmo linear que soluciona o problema do desenho retangular utilizando programação dinâmica. No capítulo 11 apresentamos dois algoritmos para encontrar as coordenadas dos vértices de um desenho retangular quando as orientações das arestas já estão definidas. Um dos algoritmos foi desenvolvido neste trabalho.

Finalmente, no capítulo 12 apresentamos algumas conclusões obtidas neste trabalho e sugerimos alguns tópicos que podem ser desenvolvidos futuramente.

No apêndice, apresentamos de forma detalhada uma implementação do algoritmo apresentado no capítulo 5 para encontrar um grafo dos caminhos dirigidos. 


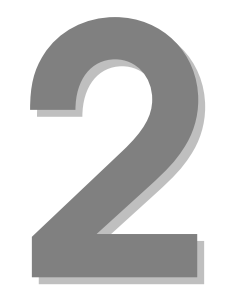

\section{CONCEITOS E DEFINIÇÕES BÁSICAS}

Um grafo $\mathbb{G}$ é um par ordenado $(V(\mathbb{G}), E(\mathbb{G}))$, onde $V(\mathbb{G})$ é o conjunto dos vértices de $\mathbb{G}$ e $E(\mathbb{G})$ é o conjunto das arestas de $\mathbb{G}$, onde $E(\mathbb{G}) \subseteq V(\mathbb{G})^{2}$. Denotamos uma aresta pelo par $(u, v)$, ou simplesmente por $u v$, onde $u, v \in V(\mathbb{G})$. Ainda, dizemos que $u$ é adjacente a $v$ se a $\operatorname{aresta}(u, v) \in E(\mathbb{G})$. Se $\alpha \in E(\mathbb{G})$, então se diz que $\alpha$ é incidente a $u$ e a $v$.

Dizemos que o grafo é não orientado, quando não importa a ordem do par $(u, v)$. Caso contrário, dizemos que o grafo é orientado ou dirigido. Ademais, vamos chamar um par $(u, v)$ no grafo orientado de arco. Seja $\alpha=(u, v)$ um arco, dizemos que o arco $\alpha$ sai do vértice $u$ e entra no vértice $v$.

Em um grafo não orientado, o grau de um vértice $v$, denotado por $d(v)$, é o número de arestas que incidem em $v$, onde os laços são contados duas vezes. Se não for claro qual é o grafo a que estamos nos referindo, usaremos $d_{\mathbb{G}}(v)$, para denotar o grau do vértice $v$ no grafo $\mathbb{G}$. Um vértice de grau 0 é chamado de isolado. No caso de grafos orientados, o grau de entrada $\left(d^{+}(v)\right)$ é o número de arestas que entram em $v$, enquanto o grau de saída $\left(d^{-}(v)\right)$ é o número de arestas que saem de $v$.

Podemos estender as definições anteriores para um conjunto de vértices.

Primeiramente, seja $\mathbb{G}$ um grafo não orientado e $X \subseteq V(\mathbb{G})$. A vizinhança de $X$ é um conjunto que contém todos os vértices de $\bar{X}=V(\mathbb{G}) \backslash X$ que são adjacentes a algum vértice pertencente a $X$. Um corte $K=(X ; \bar{X})$, denotado simplesmente por $\delta(X)$, é o conjunto de 
arestas de $\mathbb{G}$ de modo que uma aresta $u v \in K$ se e somente se $u \in X$ e $v \in \bar{X}$.

Em um grafo orientado, $\delta^{-}(X)$ denota todas as arestas que saem em $X$, ou seja, uma aresta $u v \in \delta^{-}(X)$ se $u \in X$ e $v \in \bar{X}$. De modo similar, $\delta^{+}(X)$ denota todas as arestas que entram em $X$.

Sejam $\mathbb{G}$ e $\mathbb{H}$ dois grafos quaisquer. Dizemos que $\mathbb{G}$ e $\mathbb{H}$ são isomorfos se existem uma bijeção $\phi: V(\mathbb{G}) \rightarrow V(\mathbb{H})$ tal que $u v \in E(\mathbb{G}) \Leftrightarrow \phi(u) \phi(v) \in E(\mathbb{H})$.

Para ilustrarmos o isomorfismo entre dois grafos, precisamos definir $\phi$ de modo que satisfaça a definição de isomorfismo. Para que os grafos $\mathbb{G}$ e $\mathbb{H}$ ilustrados na figura 2.1 sejam isomorfos, considere o seguinte mapeamento:

$$
\phi=\left(\begin{array}{llll}
a & b & c & d \\
w & z & y & x
\end{array}\right) .
$$

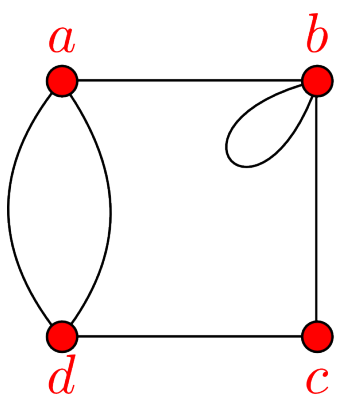

$G$

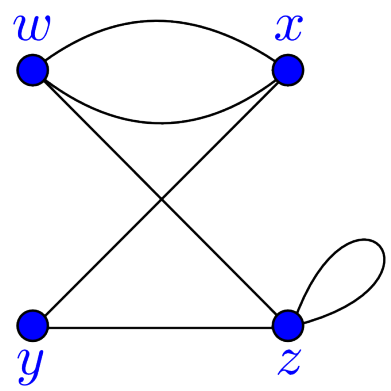

$H$

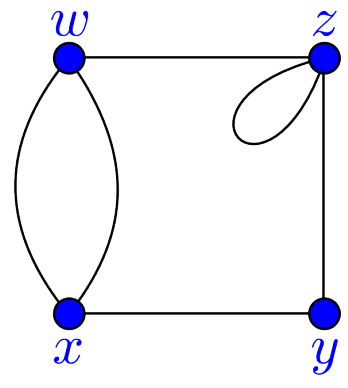

$H$

Figura 2.1: Grafos isomorfos.

Um grafo completo é um grafo em que quaisquer dois de seus vértices distintos são adjacentes. Se não considerarmos grafos isomorfos, existe um único grafo completo com $n$ vértices, denotado por $K_{n}$. O grafo $K_{3}$ também é chamado de triângulo.

Dizemos que $\mathbb{G}^{\prime}=\left(V^{\prime}, E^{\prime}\right)$ é um subgrafo induzido de $\mathbb{G}$ se e somente se:

$$
\begin{aligned}
& V\left(\mathbb{G}^{\prime}\right) \subseteq V(\mathbb{G}) \\
& E\left(\mathbb{G}^{\prime}\right)=\left\{(x, y) \mid \text { Para quaisquer } x, y \in V^{\prime} \text {, temos que }(x, y) \in E(\mathbb{G})\right\}
\end{aligned}
$$

Denotamos por $\mathbb{G}\left[V^{\prime}\right]$ o subgrafo de $\mathbb{G}$ induzido pelo conjunto de vértices $V^{\prime}$.

Seja $\mathbb{G}$ um grafo e $\mathbb{G}_{1}$ e $\mathbb{G}_{2}$ dois subgrafos de $\mathbb{G}$. A união dos grafos $\mathbb{G}_{1}$ e $\mathbb{G}_{2}$, denotada por $\mathbb{G}_{1} \cup \mathbb{G}_{2}$, resulta em um grafo $\mathbb{H}$, onde $V(\mathbb{H})=V\left(\mathbb{G}_{1}\right) \cup V\left(\mathbb{G}_{2}\right)$ e $E(\mathbb{H})=E\left(\mathbb{G}_{1}\right) \cup E\left(\mathbb{G}_{2}\right)$. Claramente, $\mathbb{H}$ também é um subgrafo de $\mathbb{G}$. 
Um passeio em um grafo é uma sequência finita não vazia de vértices $P=\left\langle v_{0}, v_{1}, \ldots, v_{k}\right\rangle \mathrm{i}$, tal que para cada dois vértices consecutivos, $v_{i}$ e $v_{i+1}$, para $0 \leq i<k$, temos que $\left(v_{i}, v_{i+1}\right) \in E(\mathbb{G})$. Dizemos que $P$ é um passeio de $v_{0}$ a (para) $v_{k}$, e $P$ passa pelos vértices $v_{i}$ e arestas $\left(v_{i}, v_{i+1}\right)$, onde $0 \leq i<k$. O vértice $v_{0}$ é o vértice inicial, enquanto o vértice $v_{k}$ é o vértice final de $P$. Os vértices $v_{1}, v_{2}, \ldots, v_{k-1}$ são chamados de vértices internos de $P$. O conjunto dos vértices e das arestas que definem $P$ é denotado por $V(P)$ e $E(P)$, respectivamente.

O comprimento ou tamanho de $P$, denotado por $|P|$, é o número de arestas de $P$.

Um caminho é um passeio sem vértices repetidos. Um circuito é um passeio não nulo onde seu vértice inicial e seu vértice final coincidem e todos os vértices $v_{i}$ são distintos, onde $0 \leq i<k$.

A concatenação de dois caminhos $A=\left\langle u_{0}, u_{1}, \ldots, u_{n}\right\rangle$ e $B=\left\langle u_{n}=v_{0}, v_{1}, \ldots, v_{m}\right\rangle$, é denotada por $A \cdot B$. O caminho resultante de $A \cdot B$ é o caminho $\left\langle u_{0}, u_{1}, \ldots, u_{n}, v_{1}, \ldots, v_{m}\right\rangle$.

Neste trabalho, em alguns momentos iremos utilizar o termo passeio (caminho, circuito) para denotar um grafo ou subgrafo cujos vértices e arestas pertencem ao passeio (caminho, circuito).

Um grafo é conexo se para todo par de vértices distintos $u, v$ existe um caminho de $u$ a $v$. Um grafo que não é conexo é chamado de desconexo. Os subgrafos conexos maximais de um grafo são chamados de componentes conexas, ou simplesmente de componentes.

Para $V^{\prime} \subseteq V(\mathbb{G}), \mathbb{G}-V^{\prime}$ é o grafo resultante da exclusão de todos os vértices que pertencem a $V^{\prime}$ de $\mathbb{G}$, juntamente com as arestas que incidem em algum vértice de $V^{\prime}$.

Seja $\mathbb{G}$ um grafo conexo e $v$ um vértice de $\mathbb{G}$. Dizemos que $v$ é uma articulação se $\mathbb{G}-\{v\}$ possui mais componentes do que $\mathbb{G}$.

Seja $\alpha=(u, v)$ uma aresta de um grafo $\mathbb{G}$. Uma subdivisão de $\alpha$ consiste na operação de remover $\alpha$ de $\mathbb{G}$ e adicionar um caminho $\left\langle u=w_{0}, w_{1}, \ldots, w_{k}, v=w_{k+1}\right\rangle$, juntamente com os vértices $w_{1}, \ldots, w_{k}$, onde $k>1$ a $\mathbb{G}$. Podemos utilizar o conceito de subdivisão para relacionar dois grafos. Um grafo $\mathbb{G}^{\prime}$ é uma subdivisão de um grafo $\mathbb{G}$ se $\mathbb{G}^{\prime}$ é obtido a partir de $\mathbb{G}$ realizando algumas subdivisões das arestas de $\mathbb{G}$.

Define-se como contração de uma aresta uv de $\mathbb{G}$, como sendo a remoção dos vértices $u$ e $v$ e a adição do vértice $w$ a $\mathbb{G}$. Ainda, para cada vértice $z$ que era vizinho a $u$ ou $v$, adicionamos a aresta $w z$ a $\mathbb{G}$. Em alguns momentos podemos nos referir a contração de um caminho $\left\langle v_{1}, \ldots, v_{k}\right\rangle$. Neste caso, considere que será realizado a contração de cada uma das arestas deste caminho. A figura 2.2 ilustra a contração das arestas do grafo de Petersen de modo que o grafo resultante seja um $K_{5}$.

Um grafo é planar se existe uma imersão no plano de modo que quaisquer duas arestas 

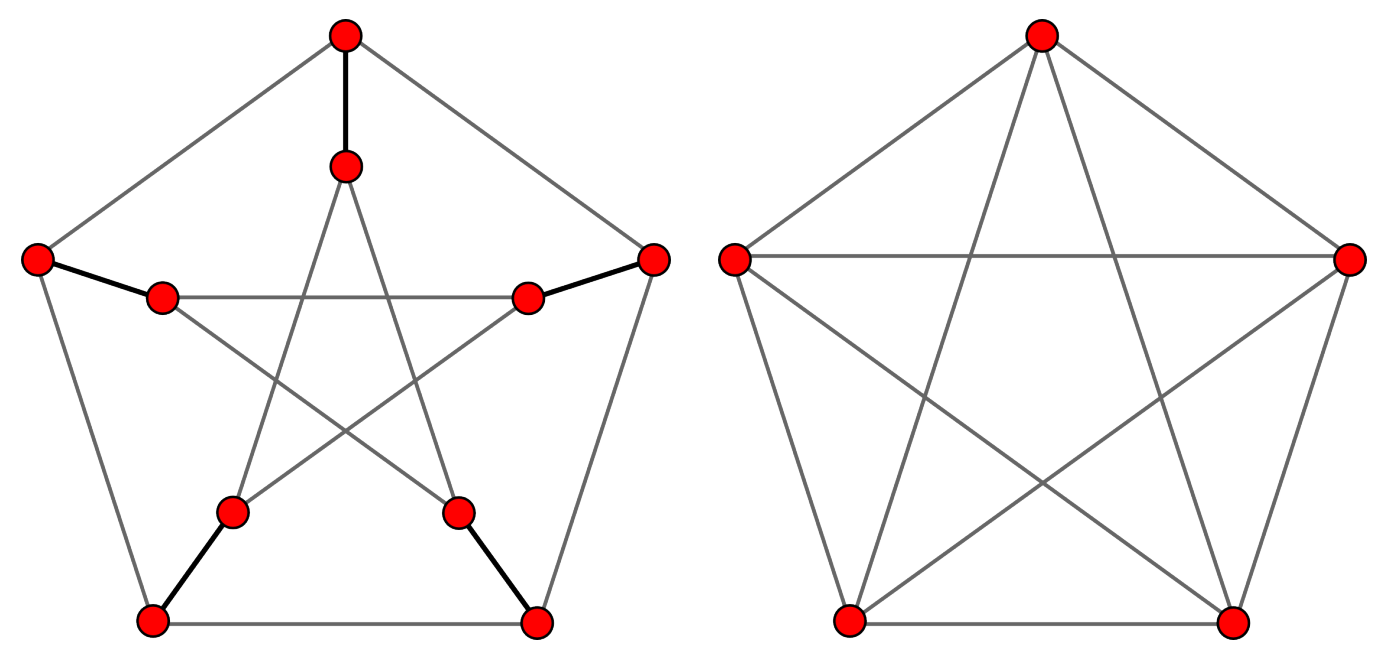

Figura 2.2: Contrações das arestas do grafo de Petersen resultando o grafo $K_{5}$.

não possuem interseção geométrica, exceto no vértice que em estas possam ser incidentes. Um grafo é plano quando existe uma imersão fixa, de modo que divide o plano em regiões conhecidas como faces. A face do grafo que é ilimitada será chamada de face externa, enquanto as outras faces serão chamadas de faces internas. Iremos identificar a face externa de $\mathbb{G}$ como $f_{\infty}$. A figura 2.3 ilustra diferentes imersões para um grafo planar $\mathbb{G}$, onde $V(\mathbb{G})=\{a, b, c, d, e\}$ e $E(\mathbb{G})=\{(a, b),(a, c),(b, c),(c, e),(b, d)\}$.
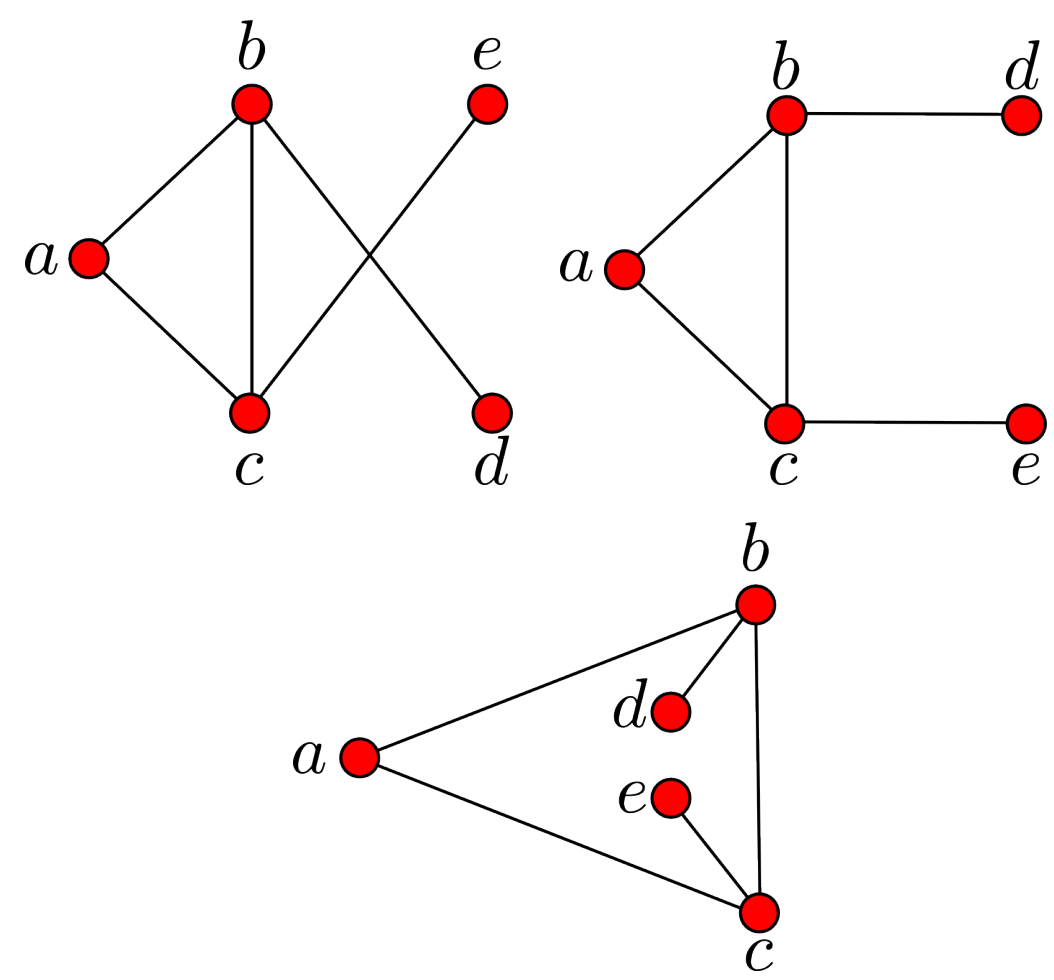

Figura 2.3: Imersões distintas de um grafo planar.

Existe uma caracterização dos grafos planares em termos de grafos proibidos. 
Teorema 2.1 (Kuratowski [Kur30]): Um grafo é planar se e somente se não contém nenhuma subdivisão de um $K_{3,3}$ ou um $K_{5}$.

Em algum momento, precisamos induzir uma ordem das arestas incidentes em uma determinada face. Vamos supor que as arestas incidentes a uma face estão descritas no sentido horário. Ainda, quando dizemos que estamos percorrendo as arestas da face, estamos percorrendo as arestas do circuito que define esta face.

Um grafo plano é triangulado se todas suas faces internas são triângulos, ou seja, são definidas por circuitos de três arestas. Caso a face externa também seja um triângulo, então o grafo é chamado de triangular.

Em um grafo plano $\mathbb{G}$, denotamos por $F(\mathbb{G})$ o conjunto das faces de $\mathbb{G}$, e por $F_{T}(\mathbb{G})$ o conjunto das faces triangulares de $\mathbb{G}$.

Seja $\mathbb{G}$ um grafo plano. Podemos definir um grafo $\mathbb{G}^{*}$ dual a $\mathbb{G}$ da seguinte forma. Para cada face $f$ de $\mathbb{G}$, existe um vértice $f^{*}$ correspondente a $f$ em $\mathbb{G}^{*}$ e para cada aresta $\alpha$ pertencente a $\mathbb{G}$, existe uma aresta $\alpha^{*}$ correspondente a $\alpha$ pertencente a $\mathbb{G}^{*}$. Ainda, existe uma aresta $\alpha^{*}=\left(f^{*}, g^{*}\right)$ em $\mathbb{G}^{*}$ se e somente se as respectivas faces $f$ e $g$ são separadas pela aresta $\alpha$ em $\mathbb{G}$. É fácil ver que o grafo $\mathbb{G}^{*}$ é um grafo plano. A figura 2.4A ilustra um grafo plano $\mathbb{G}$ e a figura $2.4 \mathrm{~B}$ ilustra o grafo dual $\mathbb{G}^{*}$. Na figura $2.4 \mathrm{C}$ podemos visualizar o grafo $\mathbb{G}$ e o seu grafo dual $\mathbb{G}^{*}$ sobrepostos. 


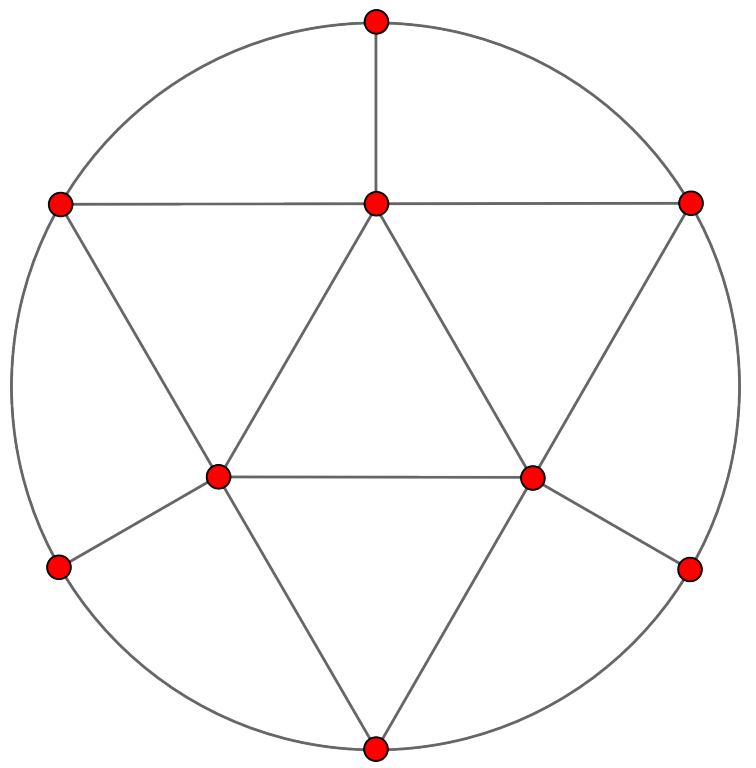

(A)

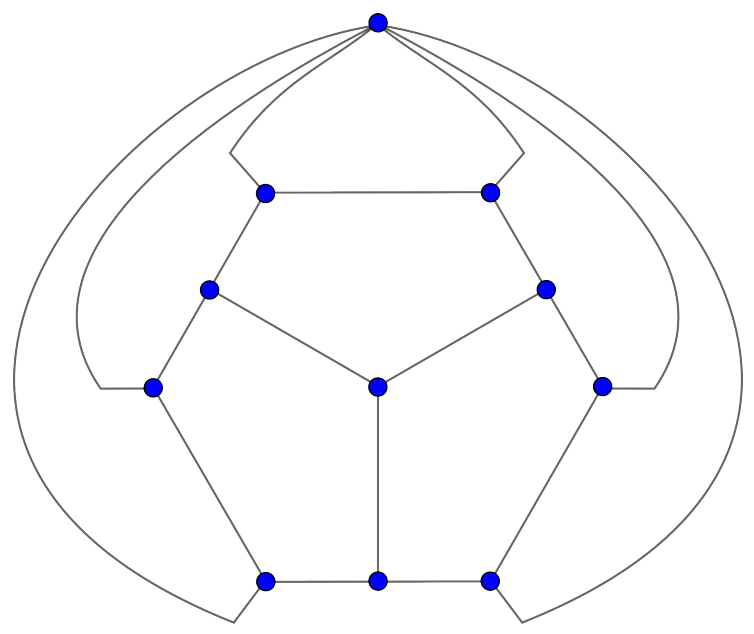

(B)

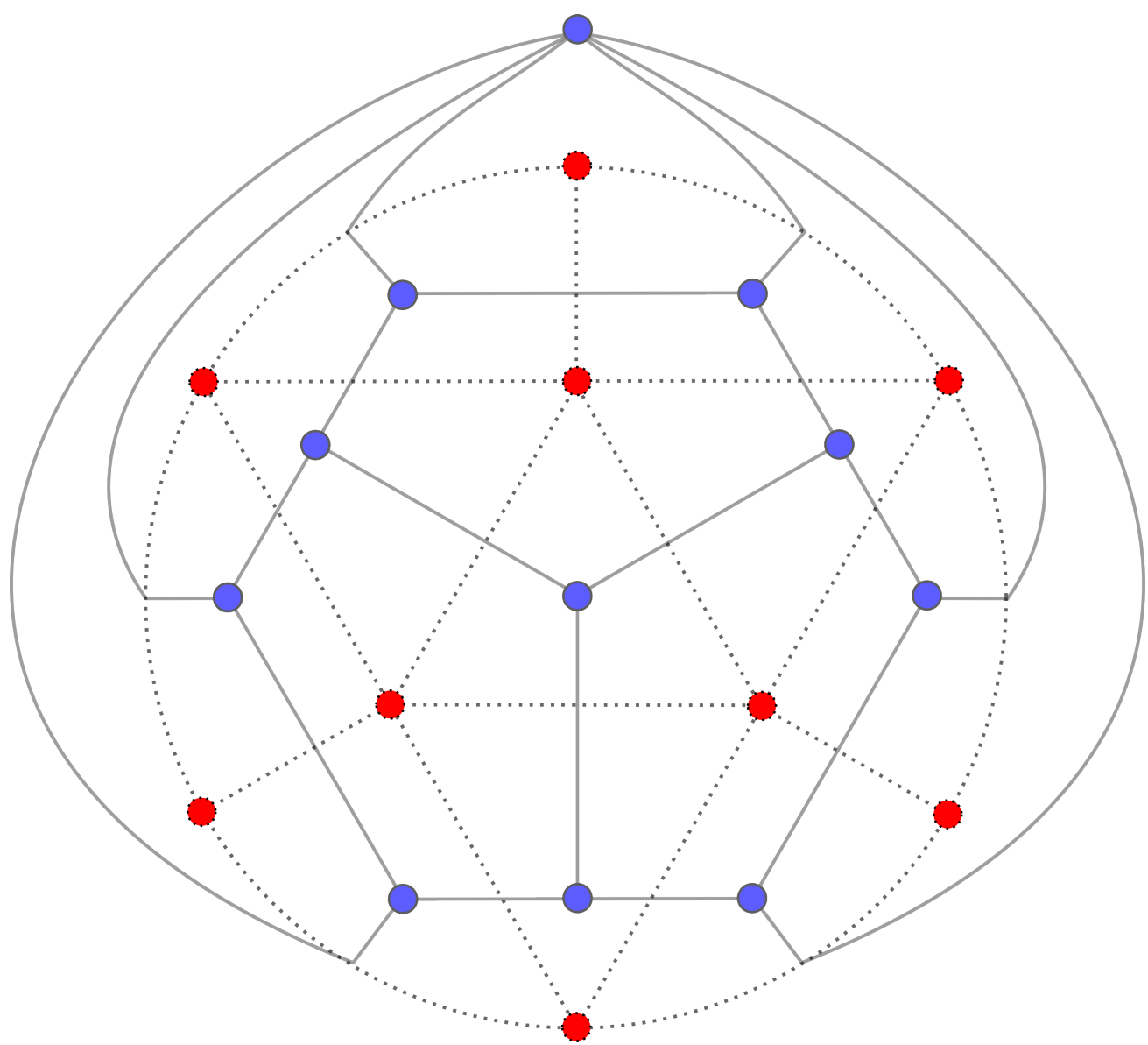

(C)

Figura 2.4: (A) Grafo plano $\mathbb{G}$. (B) Grafo $\mathbb{G}^{*}$ dual a $\mathbb{G}$. 


\section{Parte I}

\section{Problema da Representação Retangular}





\section{3 \\ Problema DA REPRESENTAÇÃO RETANGULAR}

Muitos problemas podem ser modelados utilizando grafos planares, por exemplo, o projeto de circuitos VLSI. Em um projeto de circuito VLSI desejamos decidir onde posicionar os componentes eletrônicos e como devemos desenhar os fios que conectam estes componentes de modo que estes não se cruzem. Para solucionar este problema gostaríamos que dois componentes que devem estar diretamente conectados estejam próximos no desenho. Para simplificar o problema, vamos considerar que cada componente possui forma retangular e dois componentes que estão conectados devem compartilhar algum segmento de seus lados.

Uma forma de representar as restrições de adjacências entre os componentes do circuito é construir um grafo de modo que cada componente do circuito é um vértice e cada conexão entre dois componentes é uma aresta.

Desejamos encontrar uma representação deste grafo no plano de modo que os componentes são representados por retângulos paralelos aos eixos. Os retângulos devem compartilhar algum segmento de suas bordas se e só se os vértices correspondentes no grafo são ligados por uma aresta. Ainda, gostaríamos que a figura final seja um retângulo e que este retângulo não possua buracos, ou seja, todo ponto no interior deste pertença a um retângulo de algum de seus componente. Esta representação será chamada de representação retangular do grafo dado. Também gostaríamos de decidir se um dado grafo possui uma 
representação retangular e caso possua, queremos saber como construí-la. Estes problemas serão chamados, respectivamente, de problema de decisão da representação retangular e de problema da construção da representação retangular.

Apresentamos neste capítulo os principais resultados encontrados na literatura para os problemas relacionados a representações retangulares. Este capítulo está organizado da seguinte forma. Inicialmente, definimos formalmente o problema de decisão relacionado à representação retangular. Depois, fazemos um levantamento bibliográfico dos trabalhos que sugerem uma abordagem para o problema de representação retangular.

\subsection{Definição do Problema}

Primeiramente, precisamos definir formalmente o que é uma representação retangular de um grafo plano. Iremos apresentar duas definições, uma baseada em conceitos geométricos e outra baseada em grafos.

Seja $\mathbb{R}$ um retângulo. Um sistema de subdivisão retangular $\Phi$ de $\mathbb{R}$ é uma partição de $\mathbb{R}$ em um conjunto $\Phi=\left\{R_{1}, R_{2}, \ldots, R_{n}\right\}$ de retângulos tais que a interseção do interior de quaisquer dois retângulos de $\Phi$ é vazia e não existem quatro elementos que compartilham o mesmo ponto. Uma representação retangular de um grafo plano é um sistema de subdivisão retangular onde cada elemento de $\Phi$ representa um vértice de $\mathbb{G}$ e para dois elementos, $R_{i}$ e $R_{j}$, estes compartilham algum segmento de suas bordas se e só se existe uma aresta entre os respectivos vértices representantes em $\mathbb{G}$.

Define-se grafo retangular como sendo um grafo plano onde as arestas são verticais ou horizontais, toda face interna é um retângulo e as arestas incidentes na face externa formam um retângulo. Dado um sistema de subdivisão retangular, podemos obter um grafo retangular considerando cada canto como um vértice e cada lado como uma aresta. Este fato é ilustrado na figura 3.1.

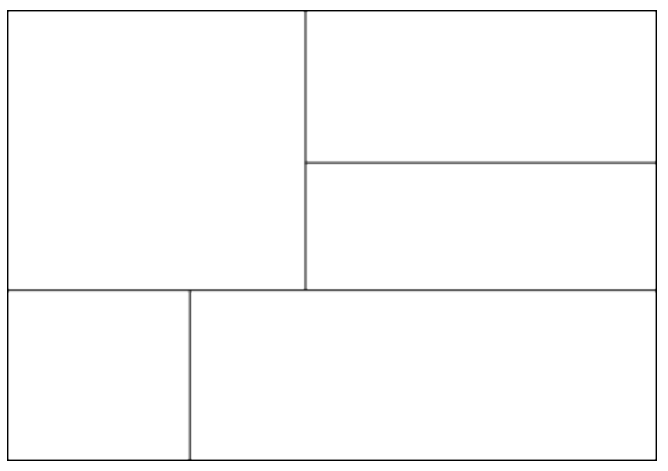

(A) Sistema de subdivisão retangular $(\Phi)$

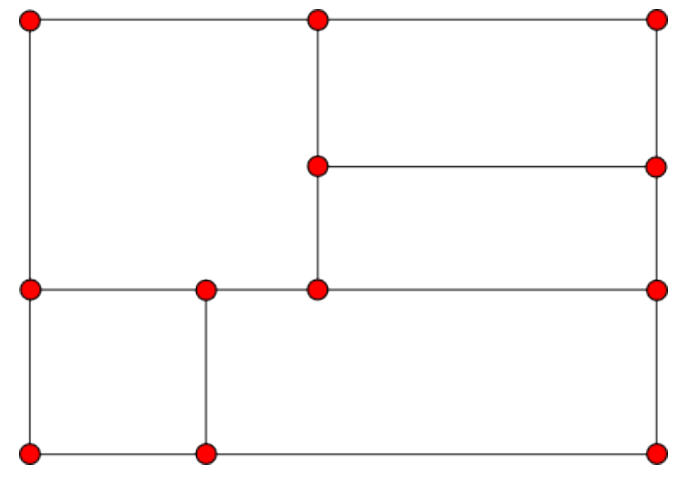

(B) Grafo retangular equivalente a $\Phi$

Figura 3.1: Transformação do sistema de subdivisão retangular em um grafo retangular 
Considere um sistema de subdivisão retangular que permite que um ponto pode pertencer a quatro retângulos, denominamos esta definição como sistema de subdivisão retangular relaxado. Note que os grafos obtidos a partir da primeira definição de sistema de subdivisão apresentada formam um subconjunto dos grafos obtidos pelo sistema de subdivisão retangular relaxado, pois a definição relaxada admite que quatro retângulos compartilhem o mesmo ponto, ou em outras palavras, permite que um vértice do grafo retangular possua grau igual a 4 . A figura 3.2 ilustra este fato.
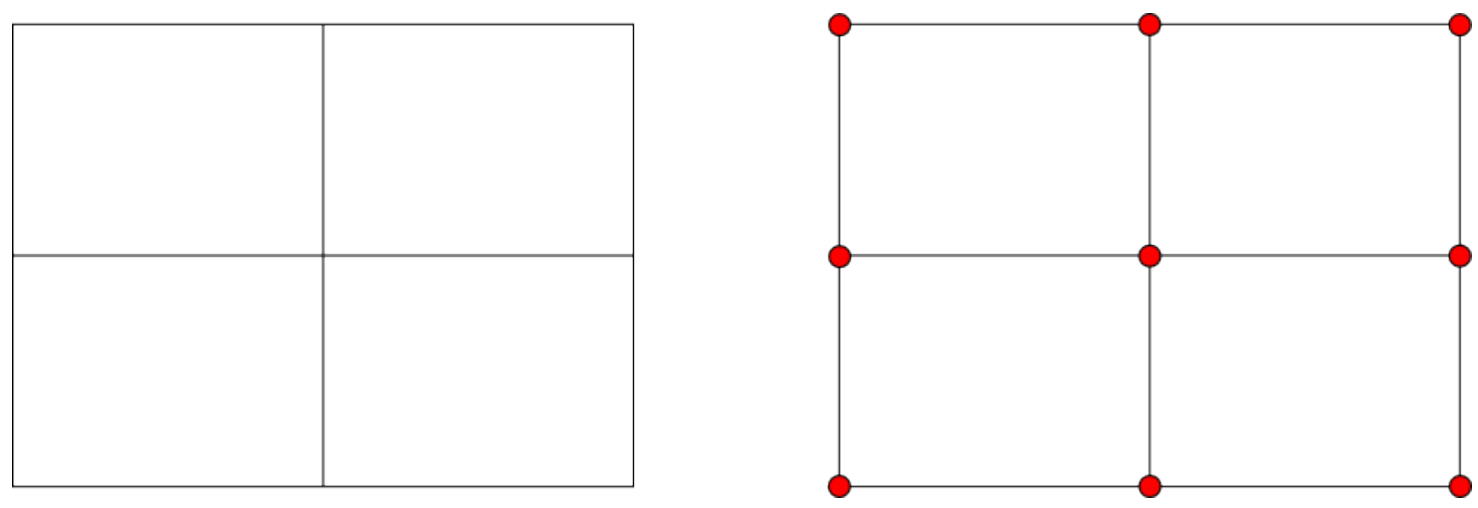

Figura 3.2: Exemplo de um sistema de subdivisão retangular relaxado e do respectivo grafo retangular que contém um vértice de grau 4

Gostaríamos que ambas definições fossem equivalentes. Para isso, vamos observar que dado um grafo com vértices de grau 4 é possível obter um grafo equivalente em que todos os vértices têm grau menor ou igual a 3 subdividindo-se os vértices de grau 4 em dois ligados por uma aresta artificial.

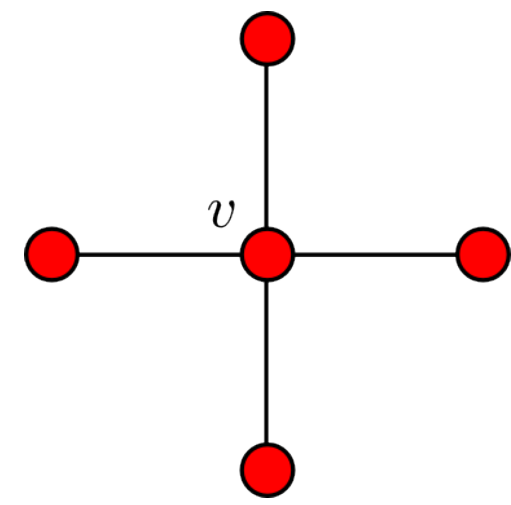

(A) Vértice de grau 4

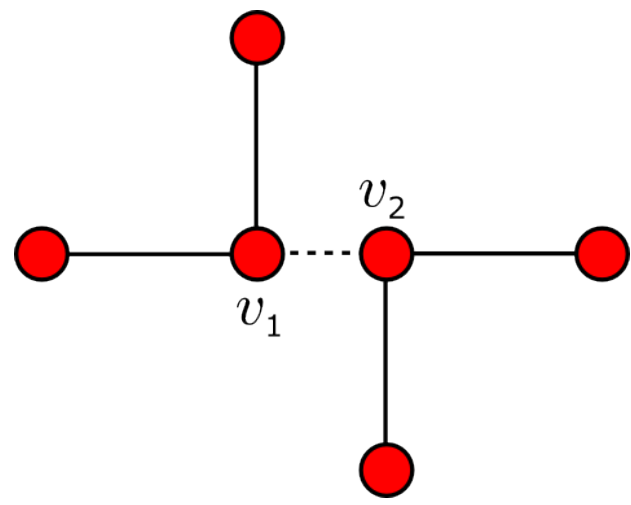

(B) Vértices de grau 3 resultantes

Figura 3.3: Transformação de vértices de grau 4 em vértices de grau 3

O processo inverso da transformação é trivial. Basta contrair os vértices que incidem em uma aresta artificial e unir as arestas não artificiais que incidem em ambos os vértices.

Daqui para frente sempre que nos referirmos a um grafo retangular estamos supondo que seu grau máximo é 3, ou seja, iremos considerar que estamos trabalhando com um sistema de subdivisão retangular. 
Assim, podemos enunciar agora um dos problemas de interesse deste trabalho.

\section{Problema 1. Problema de Decisão DA REPRESEntaÇÃo RETANGUlar}

Entrada: $\quad$ Grafo plano $\mathbb{G}=(V, E)$.

SAIDA: Decidir se dado um grafo plano $\mathbb{G}$ existe uma representação retangular $\mathbb{R}$ de $\mathbb{G}$.

Consequentemente, podemos relacionar ao problema de decisão um problema de construção.

\section{Problema 2. Problema da COnstruÇÃo da RePresentaÇÃo Retangular}

Entrada: $\quad$ Grafo plano $\mathbb{G}=(V, E)$.

SAIDA: Dado um grafo plano $\mathbb{G}$, construir, caso exista, uma representação retangular $\mathbb{R}$ de $\mathbb{G}$.

Agora, vamos mostrar que, com exceção dos casos triviais onde o grafo de entrada contém no máximo dois vértices, o grafo de entrada $\mathbb{G}$ dos problemas 1 e 2 deve ser triangulado.

Lema 3.1: Todo grafo com pelo menos três vértices e que admite uma representação retangular é triangulado.

Demonstração: Suponha por absurdo que o grafo $\mathbb{G}$ não é triangulado, e que existe um sistema de subdivisão retangular $\Phi$ e uma bijeção entre os vértices de $\mathbb{G}$ e os elementos de $\Phi$.

Suponha que existe uma face $f$ é limitada por 2 arestas. É fácil ver que temos uma contradição com o fato da representação retangular possuir vértices de grau 2 que não são cantos (Figura 3.4). Suponha que exista uma face $f$ limitada por mais de 3 arestas. Por definição, sabemos que não existe um vértice que pertença a quatro retângulos em qualquer sistema de subdivisão retangular. Assim, para representarmos as restrições de adjacências induzida pela face $f$ necessitaríamos criar um buraco. Pois, como cada retângulo só possui dois retângulos adjacentes alguma parte de seus segmentos não serão compartilhados com nenhum outro retângulo. A figura 3.5 ilustra este fato para uma face limitada por 4 arestas.

Portanto, para grafos planos não triviais temos que $\mathbb{G}$ é um grafo plano triangulado.

Um exemplo do grafo de entrada dos problemas 1 e 2 é ilustrado na figura 3.6A. Na figura 3.6B ilustramos a solução do problema 2 para o grafo de entrada apresentado na figura 3.6A.

Estes problemas são bem resolvidos, uma vez que existe um conjunto de condições suficientes e necessárias que caracterizam bem cada um destes problemas. 


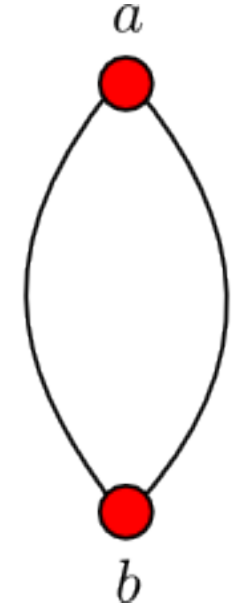

(A) Ilustração de uma face limitada por duas arestas

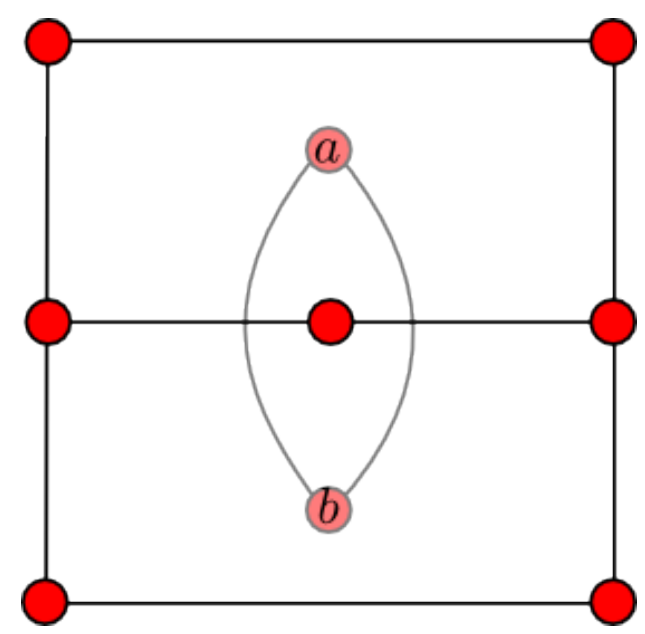

(B) Ilustração de uma representação retangular da face de $(A)$

Figura 3.4: Ilustração do lema 3.1 quando uma face do grafo plano da entrada é definida por duas arestas.

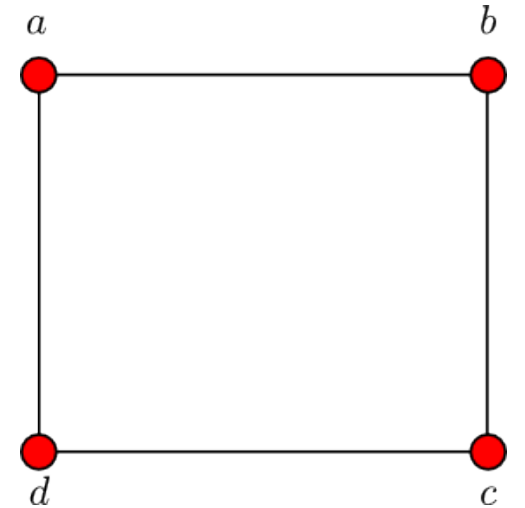

(A) Ilustração de uma face limitada por 4 arestas

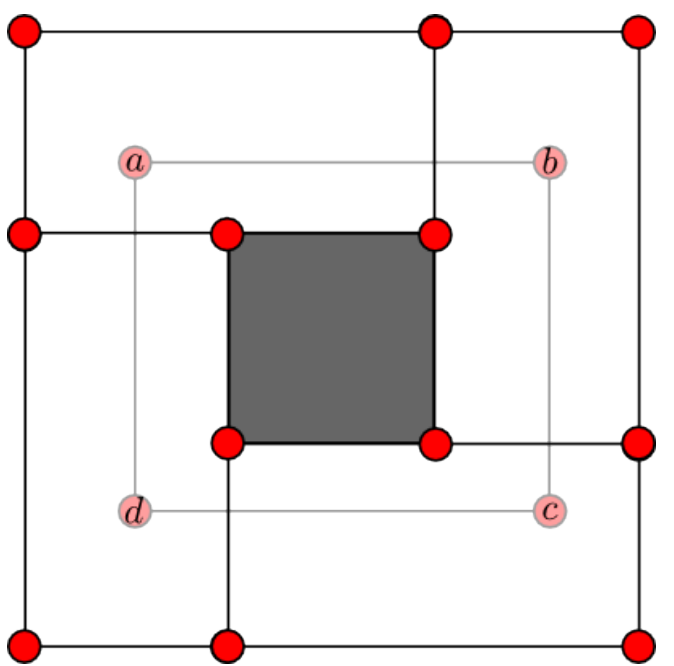

(B) Ilustração de uma representação retangular da face de (A). A região em cinza representa um retângulo que não pertence a nenhum dos vértices do grafo.

Figura 3.5: Ilustração do lema 3.1 quando uma face do grafo plano da entrada é definida por quatro arestas.

\subsection{REVISÃo BibLIOGRÁFICA}

Os problemas que envolvem representações retangulares são solucionados por algoritmos eficientes que utilizam abordagens distintas. Primeiramente, foi apresentada uma caracterização dos grafos que possuem representações retangulares. Após este resultado, surgiram algoritmos para solucionar os problemas de forma eficiente.

Um dos principais resultados para a caracterização da existência de representações 


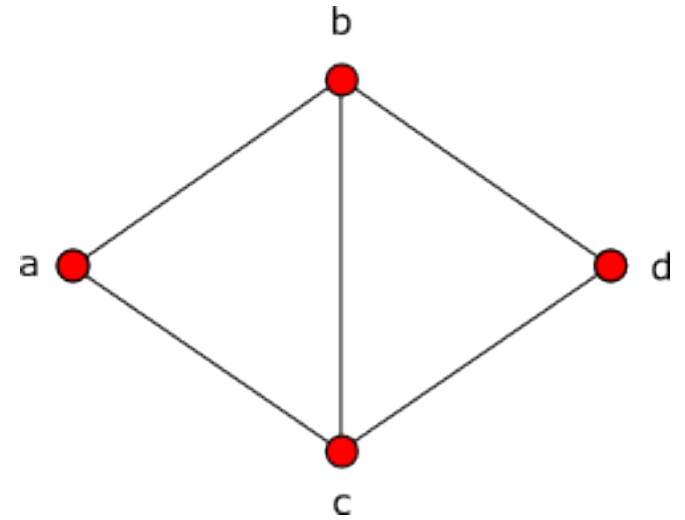

(A)

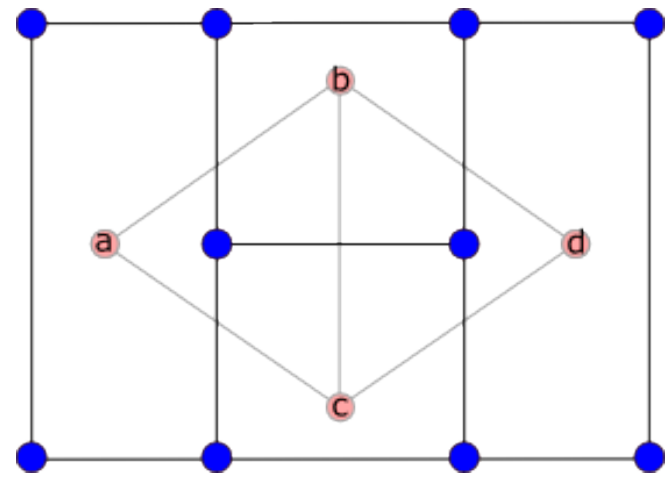

(B)

Figura 3.6: Ilustração da entrada e saída do problema de representação retangular. (A) Grafo de Entrada. (B) Uma representação retangular do grafo ilustrado em (A).

retangulares é devido a Koźmiński e Kinnen [KK84, KK85]. Baseados em sua caracterização, Koźmiński e Kinnen[KK84] apresentam um algoritmo $O\left(n^{2}\right)$, onde $n$ é o número de vértices do grafo, para encontrar uma representação retangular do grafo dual, caso esta existe. Caso contrário o algoritmo retorna um certificado da inexistência de uma representação retangular.

Em [BS87], Bhasker e Shani desenvolvem um algoritmo de complexidade linear que verifica as condições da caracterização apresentada por Koźmiński e Kinnen em [KK84]. Um algoritmo para construção da representação retangular baseado em [BS85] com complexidade linear é apresentado em [BS87].

Bhasker e Shani [BS88] apresentam um algoritmo linear para resolver o problema 2. Seu algoritmo é baseado no algoritmo apresentado em [BS87], no qual Bhasker e Shani desenvolveram um algoritmo linear para solucionar o problema 2. O algoritmo apresentado em [BS88] constrói a representação retangular do grafo da entrada a partir da esquerda para direita. Seu algoritmo possui duas etapas: (i) encontrar uma certa rotulação das arestas; e (ii) construir uma representação retangular a partir destes rótulos.

Em [He93], He utiliza algumas ideias de Lai e Leinwand [LL90] e de Bhasker e Shani [BS88] para desenvolver um algoritmo linear baseado em $s, t$-caminhos orientados. Basicamente, He partiu do fato que existe para cada aresta do grafo triangular uma aresta correspondente no grafo dual retangular. Assim, He percebeu que existe uma ordem para decidir se uma aresta será horizontal ou vertical e esta ordem é determinada pelos $s, t$ caminhos orientados. Este algoritmo possui complexidade de tempo linear e é relativamente simples para encontrar e construir a representação retangular do grafo de entrada.

Kant e He [KH97] também utilizam uma abordagem semelhante ao trabalho de Bhasker e Shani [BS88] e apresentam dois algoritmos que simplificam o passo para encontrar uma certa rotulação das arestas do grafo da entrada.

Lai e Leinwand [LL90] apresentam uma redução dos problemas 1 e 2 ao problema de 
encontrar um emparelhamento perfeito em um grafo bipartido.

Os próximos capítulos irão apresentar algumas das abordagens listadas acima para solucionar os problemas de decisão e construção da representação retangular. 



\section{4 \\ CARACTERIZAÇÃO DE GRAFOS COM REPRESENTAÇÕES RETANGULARES}

Neste capítulo, vamos apresentar a caracterização desenvolvida por Koźmiński e Kinnen [KK84, KK85] para os problemas de decisão e construção da representação retangular.

Este capítulo está organizado da seguinte forma. Inicialmente, iremos apresentar uma definição alternativa para o problema de representação retangular, depois apresentaremos a caracterização do problema e por fim um algoritmo que implementa o resultado de Koźmiński e Kinnen.

\subsection{RESUlTADO PRINCIPAL}

Koźmiński e Kinnen [KK85] apresentaram uma forma de modificar o grafo plano triangulado da entrada do problema de representação retangular a fim de que este se torne 4-conexo. A caracterização do problema de representação retangular apresentada por Koźmiński e Kinnen necessita que o grafo de entrada seja 4-conexo. A seguir, vamos apresentar tal construção.

Considere um cubo e um grafo plano triangulado. Desejamos particionar uma determinada face deste cubo a fim de encontrar um sistema de subdivisão retangular que 
satisfaça as restrições do grafo plano triangulado que foi dado. Podemos interpretar este cubo como sendo uma forma de envolver a representação retangular utilizando 4 retângulos. Este fato fica mais claro se desenharmos o cubo no plano. Note que neste desenho, a face do cubo oposta à particionada não ficará visível, para contornar este problema, vamos considerar esta face como a face externa desta representação. Iremos chamar este tipo de representação retangular de representação retangular cúbica. A figura 4.1B ilustra o cubo com uma das faces particionadas de modo que o sistema de subdivisão retangular satisfaça as restrições impostas pelo grafo da figura 4.1A. A figura 4.1C é uma outra forma de visualizar o cubo da figura 4.1B. Na figura 4.1D ilustramos como envolver a representação retangular utilizando quatro retângulos. Note que existe uma relação entre os retângulos utilizados para envolver a representação retangular e as faces do cubo da figura 4.1B e 4.1C. A face externa da figura 4.1D corresponde à face oposta da particionada no cubo. Por fim, a figura 4.2 ilustra o grafo plano triangular cuja representação retangular é a representação retangular cúbica da figura 4.1D.

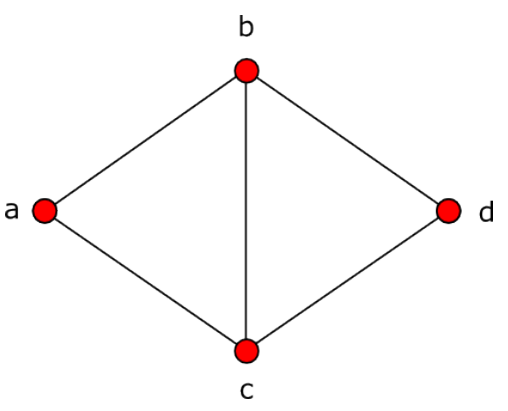

(A)

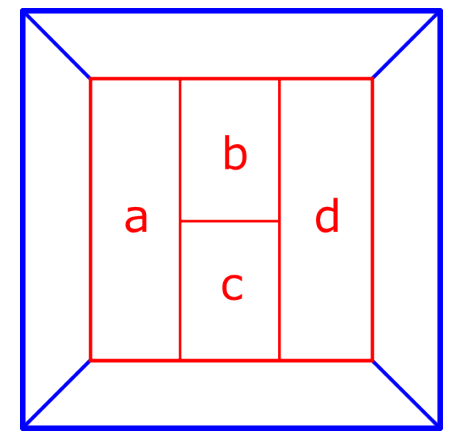

(C)

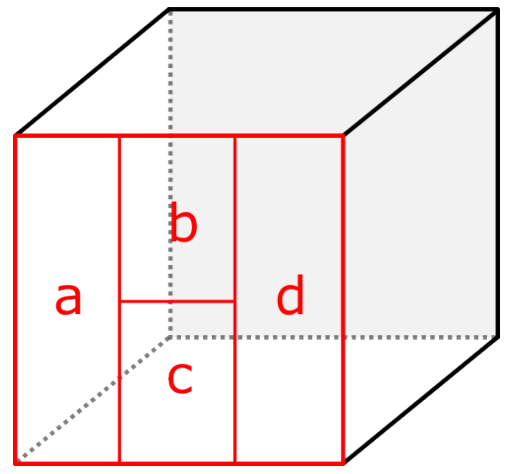

(B)

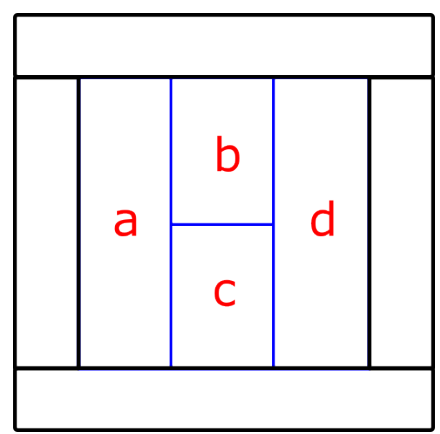

(D)

Figura 4.1: (A) Grafo Plano Triangular $\mathbb{G}$. (B) Ilustração do cubo com a face em azul particionada em retângulos. (C) Uma outra forma de representar o cubo (B). (D) Ilustração de como envolver a representação retangular.

Antes de enunciar os principais resultados do trabalho de Koźmiński e Kinnen, vamos relembrar o teorema de Menger [Men27] e para isso precisamos de alguns conceitos.

Dois caminhos em um grafo são internamente disjuntos nos vértices se os únicos vértices que estes possuem em comum são o início e o fim do caminho. Para vértices $r, s$ pertencentes 


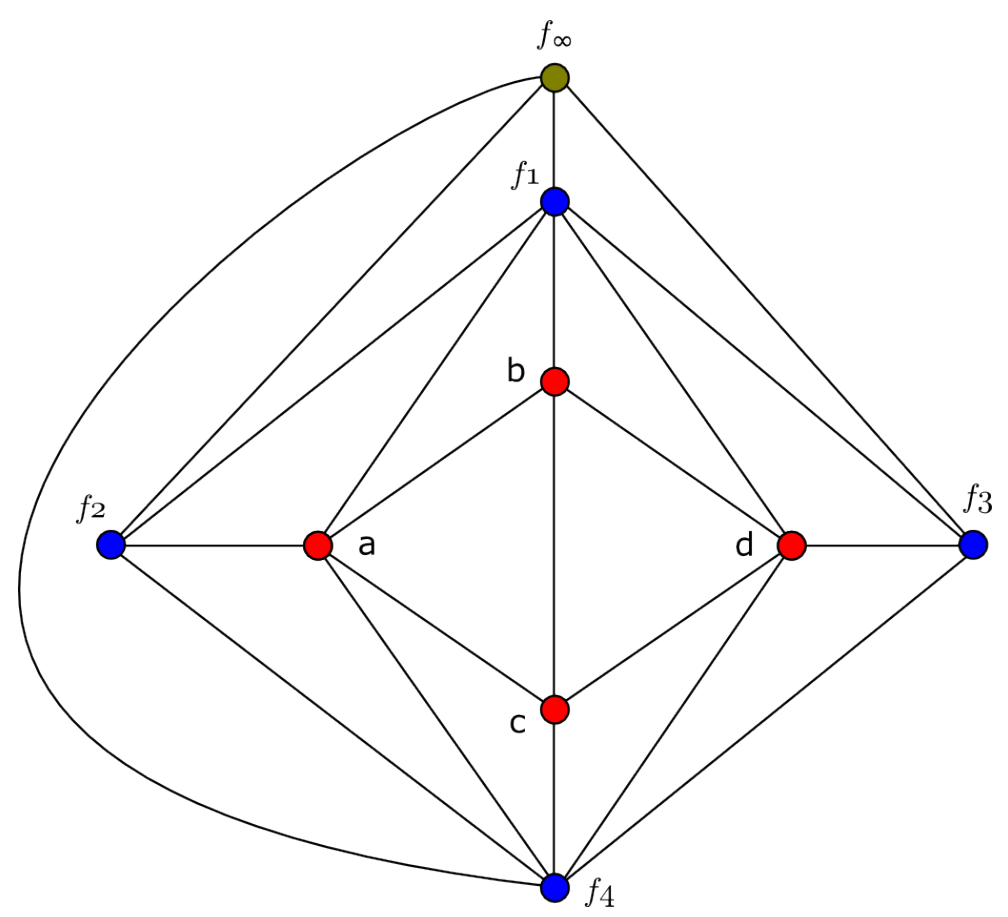

Figura 4.2: Grafo plano triangular com representação retangular dada por 4.1D.

a um grafo $\mathbb{G}$, um conjunto $S \subseteq V(\mathbb{G}) \backslash\{r, s\}$ separa $r$ de $s$ se não existe um caminho de $r$ a $s$ no grafo $\mathbb{G}-S$.

Teorema 4.1 (Menger, 1927): Seja $\mathbb{G}$ um grafo e $r, s \in V(\mathbb{G})$. O número mínimo de vértices que separam $r$ de $s$ é igual ao número máximo de caminhos internamente disjuntos nos vértices de $r$ a $s$.

Lema 4.2: Seja $\mathbb{G}$ um grafo plano, onde $n=|V(\mathbb{G})| \geq 6$. Se $\mathbb{G}$ é um grafo triangular então existe ao menos um vértice de grau 4 ou um vértice de grau 5.

Demonstração: Considere $m=|E(\mathbb{G})|$ e $f=|F(\mathbb{G})|$.

É fácil ver que não existem vértices com grau $\leq 3$. Suponha por absurdo que $\forall v \in V(\mathbb{G})$, temos que $d(v) \geq 6$.

$$
\begin{aligned}
\sum_{v \in V(\mathbb{G})} 6 \leq \sum_{v \in V(\mathbb{G})} d(v) & =2|E(\mathbb{G})| \\
6 n & \leq 2 m \\
3 n & \leq m .
\end{aligned}
$$


Por outro lado,

$$
\begin{aligned}
\sum_{x \in F(\mathbb{G})}|E(x)| & =2|E(\mathbb{G})| \\
3 f & =2 m \\
\frac{3 f}{2} & =m .
\end{aligned}
$$

Logo,

$$
\begin{aligned}
\frac{3 f}{2}=m & \geq 3 n \\
\frac{3 f}{2} & \geq 3 n \\
\frac{f}{2} & \geq n .
\end{aligned}
$$

Pela fórmula de Euler,

$$
\begin{aligned}
m-n+2 & =f \\
\frac{3 f}{2}-n+2 & =f \\
\frac{f}{2}+2 & =n \\
\frac{f}{2} & <n .
\end{aligned}
$$

Um absurdo. Logo, deve existir ao menos um vértice com grau igual 4 ou 5.

Lema 4.3: Um grafo plano triangular $\mathbb{G}$ é 4-conexo se e somente se todo triângulo (circuito com 3 vértices) é uma face.

Demonstração: Para provar a suficiência, suponha por absurdo que um grafo plano triangular $\mathbb{G}$ possui um circuito $C$ que não é uma face de modo que $|C| \leq 3$. Os vértices de $C$ definem um corte $K=(V(C) ; \overline{V(C)})$, onde $|V(C)|<4$. Note que $K$ separa os vértices internos a $C$ dos vértices externos a $C$, assim $\mathbb{G}$ não é 4-conexo.

Para provar a necessidade, suponha que $\mathbb{G}$ é um grafo plano triangular e todos os circuitos que não são faces possuem pelo menos 4 arestas. Vamos provar o resultado por indução no número de vértices $n=|V(\mathbb{G})|$ de $\mathbb{G}$.

É fácil verificar para todos os grafos triangulares com $n \leq 6$. Suponha que a hipótese vale para $n \geq 6$.

Seja $\mathbb{G}$ um grafo plano triangular contendo $n+1$ vértices de modo que todos os circuitos que não são faces possuem ao menos 4 arestas. Todos os vértices de $\mathbb{G}$ devem possuir, pelo 
menos, grau 4. Pois, se tivermos um vértice com grau menor que 4 teríamos um circuito com tamanho menor que 4 e que não é face. (Figura 4.3)

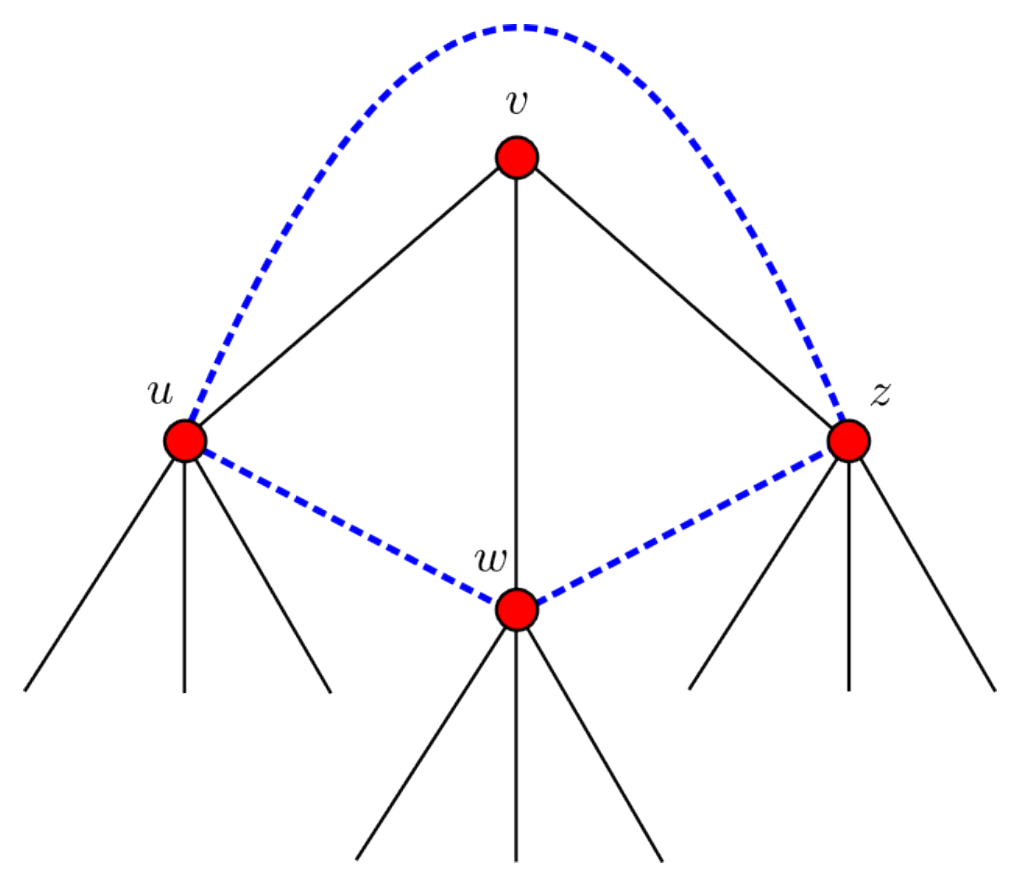

Figura 4.3: Exemplo de um grafo plano triangular, onde $d(v)=3$. O circuito que não é uma face é ilustrado pela linha pontilhada azul.

Ademais, pelo lema 4.2 existe em $\mathbb{G}$ ao menos um vértice de grau 4 ou grau 5 . Considere os casos:

Caso 1: Suponha existe um vértice $v \in V(\mathbb{G})$ tal que $d(v)=4$. Sejam $v_{1}, v_{2}, v_{3}$ e $v_{4}$ os vizinhos de $v$. Considere $\mathbb{G}^{\prime}=\mathbb{G}-v$.

Se existir um vértice $w \neq v_{2}, v_{4}$ que é adjacente a $v_{1}$ e $v_{3}$, adicione a aresta $v_{2} v_{4}$ a $\mathbb{G}^{\prime}$. Caso não exista tal vértice $w$, adicione a aresta $v_{1} v_{3}$ a $\mathbb{G}^{\prime}$.

Observe que umas das arestas $w v_{2}$ ou $w v_{4}$ devem existir para que todas as faces sejam triangulares. A existência da aresta depende de como as arestas $w v_{1}$ e $w v_{3}$ foram desenhadas. Entretanto, as figuras $4.4 \mathrm{~A}$ e $4.4 \mathrm{~B}$ ilustram o fato de que as arestas $w v_{2}$ e $w v_{4}$ não podem existir simultaneamente com as arestas $w v_{1}$ e $w v_{3}$ em um grafo plano triangular com mais de 6 vértices, pois note que o sétimo vértice será desenhado em umas das faces $F_{1}, F_{2}, F_{3}$ ou $F_{4}$. Sem perda de generalidade, suponha que o vértice foi desenhado na face $F_{1}$. Logo, o triângulo $\left\langle w, v_{4}, v_{1}, w\right\rangle$ não é face, contrariando a hipótese de que todos os circuitos que não são faces de $\mathbb{G}$ possuem tamanho maior ou igual a 4. Conforme a figura $4.4 \mathrm{C}$ ilustra, se existe a aresta $w v_{2}$, então todos outros vértices que não foram ilustrados pelo fragmento de $\mathbb{G}$, devem ser desenhados na face definida pelo circuito $\left\langle w, v_{1}, v_{4}, v_{3}, w\right\rangle$. Caso seja desenhado em outra face, teremos, ou uma contradição com o fato de $v$ possuir grau 4, ou de que todo circuito que não é face possui ao menos 4 arestas. 


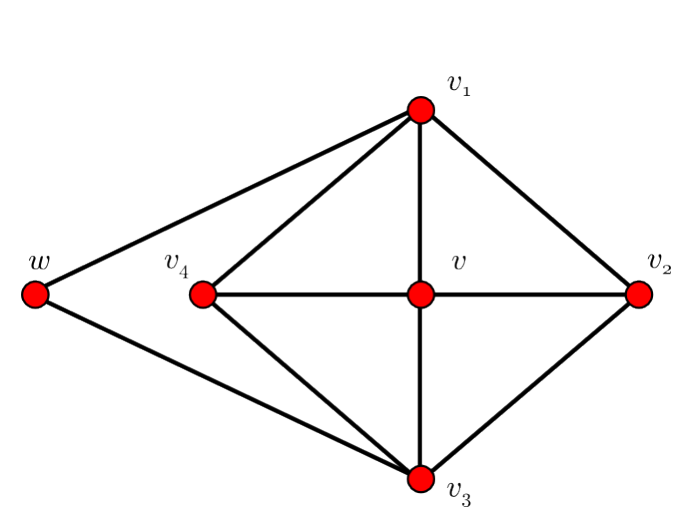

(A)

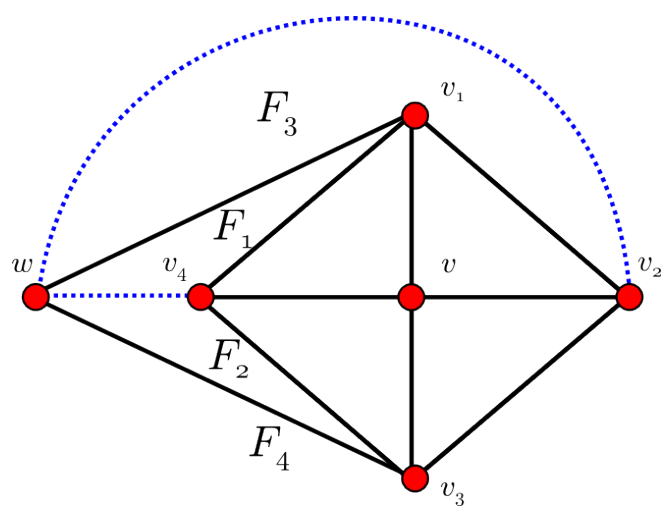

(B)

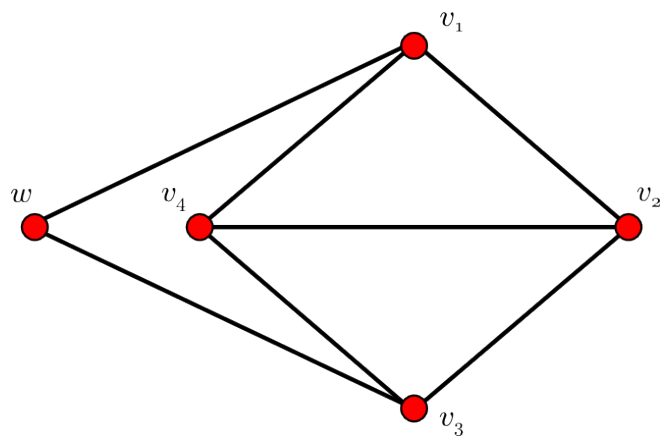

(C)

Figura 4.4: (A) Ilustração da existência de um vértice $w$ adjacente aos vértices $v_{1}$ e $v_{3}$. (B) Caso $w$ seja adjacente a $v_{2}$ e $v_{4}$ (arestas pontilhadas), existe ao menos um vértice de $\mathbb{G}$ que deve ser desenhado em uma das faces $F_{1}, F_{2}, F_{3}$ ou $F_{4}$. (C) Ilustração de um fragmento de $\mathbb{G}^{\prime}$ com $w$ adjacente a $v_{1}$ e $v_{3}$.

Sem perda de generalidade, suponha que adicionamos a aresta $v_{2} v_{4}$ e que existe a aresta $w v_{2}$. Assim, $\mathbb{G}^{\prime}$ é um grafo plano triangulado com $n$ vértices e todo circuito que não é face possui ao menos 4 arestas. Logo, pela hipótese indutiva, $\mathbb{G}^{\prime}$ é 4-conexo.

Pelo teorema de Menger, existem 4 caminhos internamente disjuntos entre quaisquer dois vértices em $\mathbb{G}^{\prime}$. Perceba que para todos os caminhos existentes em $\mathbb{G}^{\prime}$, existe um caminho equivalente em $\mathbb{G}$, pois caso um caminho em $\mathbb{G}^{\prime}$ utilizar a aresta $v_{2} v_{4}$, então basta substituir esta aresta por $v_{2} v v_{4}$. Se removermos menos que 4 vértices de $\mathbb{G}$ existirá ao menos um caminho de $v$ para qualquer outro vértice do grafo resultante. Logo, $\mathbb{G}$ é um grafo 4-conexo.

Caso 2: Suponha que não existem vértices de grau 4, assim existe um vértice $v$ tal que $d(v)=5$. Seja $N=\left\{v_{1}, v_{2}, v_{3}, v_{4}, v_{5}\right\}$ os vizinhos de $v$ em ordem horária. Suponha que existe um vértice $w \notin N$ adjacente a $x, y \in N$ de modo que $x y \notin E(\mathbb{G})$. Sem perda de generalidade, sejam estes vértices $v_{1}$ e $v_{3}$. Note que não pode existir uma aresta $w v_{2}$, caso contrário iria implicar que $v_{2}$ possui grau 4 , ou que existe um triângulo que não é uma face. Vamos mostrar uma construção similar à apresentada no caso 1. Considere $\mathbb{G}^{\prime}=\mathbb{G}-v$. Adicione as arestas $v_{2} v_{4}$ e $v_{2} v_{5}$ a $\mathbb{G}^{\prime}$. Logo, $\mathbb{G}^{\prime}$ é um grafo plano triangular. As figuras $4.5 \mathrm{~A}$ e $4.5 \mathrm{~B}$ ilustram esta construção. Caso não exista $w \in V(G)$ nestas 
condições, podemos acrescentar em $\mathbb{G}-v$ duas arestas quaisquer entre um vértice de $N$ e os dois outros que não sejam seus vizinhos. O grafo assim construído é plano triangular e todo circuito que não é face possui tamanho maior ou igual a 4.

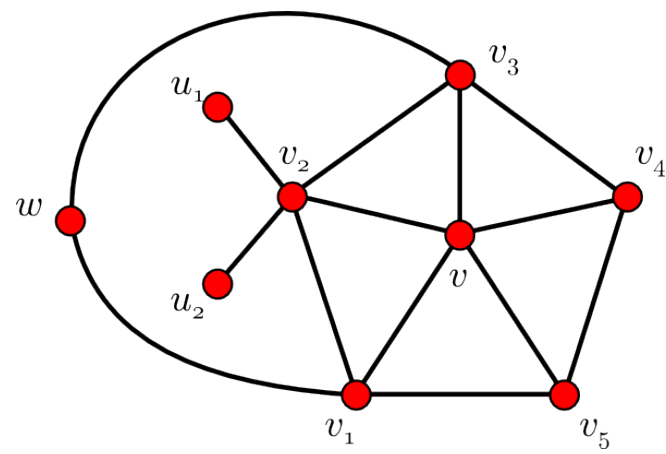

(A)

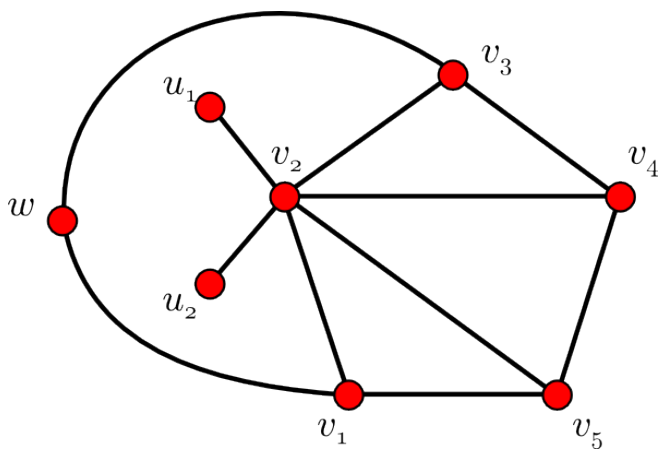

(B)

Figura 4.5: (A) Ilustração da existência de um vértice $w$ adjacente aos vértices $v_{1}$ e $v_{3}$. (B) Remoção do vértice $v$ e adição das arestas $v_{2} v_{4}$ e $v_{2} v_{5}$. [KK85]

Como $\mathbb{G}^{\prime}$ possui $n$ vértices e todo circuito que não é face possui ao menos 4 arestas, pela hipótese indutiva $\mathbb{G}^{\prime}$ é 4-conexo. Pelo teorema de Menger, para quaisquer dois vértices $u, v$ de $\mathbb{G}^{\prime}$ existem quatro caminhos internamente disjuntos que ligam $u$ a $v$. Ainda, note que existem 4 caminhos internamente disjuntos que saem dos vértices $v_{1}, v_{3}, v_{4}$ e $v_{5}$ e chegam em qualquer outro vértice de $\mathbb{G}^{\prime}$ que utilizam no máximo uma vez uma das arestas $v_{2} v_{4}$ e $v_{2} v_{5}$.

Temos que o grau de $v_{2}$ em $\mathbb{G}$ é pelo menos 5 . Assim, existem ao menos dois vizinhos de $v_{2}$ diferentes de $v, v_{1}$ e $v_{3}$, digamos $u_{1}$ e $u_{2}$. Como $\mathbb{G}^{\prime}$ é 4-conexo, então existem quatro caminhos internamente disjuntos que partem dos vértices $u_{1}$ ou $u_{2}$ e chegam nos vértices $v_{1}, v_{3}, v_{4}$ ou $v_{5}$. Ademais, é fácil ver que existem quatro caminhos internamente disjuntos de $u_{1}$ ou $u_{2}$ aos vértices $v_{1}, v_{3}, v_{4}$ ou $v_{5}$ que utilizam no máximo uma vez uma das arestas $v_{2} v_{4}$ e $v_{2} v_{5}$. Os mesmos caminhos também existem em $\mathbb{G}$, basta considerar ao invés de $v_{2} v_{4}$ as arestas $v_{2} v$ e $v v_{4}$ e no lugar de $v_{2} v_{5}$ as arestas $v_{2} v$ e $v v_{5}$.

Gostaríamos de remover 3 vértices e mostrar que não é possível separar $v$ de algum vértice de $\mathbb{G}$. Perceba que como existem quatro caminhos internamente disjuntos de $u_{1}$ ou $u_{2}$ aos vértices $v_{1}, v_{3}, v_{4}, v_{5}$ em $\mathbb{G}$. Então não conseguimos isolar $v$ de algum vértice removendo $v_{2}$. Logo, precisamos remover 3 vértices de $\mathbb{G}$ diferentes de $v_{2}$. Assim, existe ao menos um dos vértices $v_{1}, v_{3}, v_{4}, v_{5}$ que ainda é vizinho de $v$, digamos $x$ e existe ao menos um caminho de $x$ a qualquer outro vértice pertencente a $V(\mathbb{G}) \backslash\left\{v, v_{2}\right\}$. Ainda, existe algum vértice diferente de $v$ que é vizinho de $v_{2}$. Implicando assim, que se removermos menos que quatro vértices, temos que $\mathbb{G}$ é conexo. Logo, $\mathbb{G}$ é 4-conexo. 
Um grafo plano triangular 4-conexo com pelo menos um vértice de grau 4 é chamado de 4-triangulação. Note que o grafo ilustrado na figura 4.2 é uma 4-triangulação.

Lema 4.4: Se $\mathbb{G}$ admite uma representação retangular cúbica então $\mathbb{G}$ é uma 4-triangulação.

Demonstração: Para mostrarmos que $\mathbb{G}$ é uma 4-triangulação precisamos mostrar que $\mathbb{G}$ é 4-conexo e que existe ao menos um vértice de grau 4.

O vértice de $\mathbb{G}$ que representa a face externa da representação retangular cúbica tem grau 4. Agora, precisamos mostrar que $\mathbb{G}$ é 4-conexo, ou seja, após a remoção de quaisquer 3 vértices de $\mathbb{G}$ temos que o grafo resultante continua conexo. Vamos denotar os 4 vértices correspondentes aos retângulos externos da representação retangular cúbica por $v_{S}, v_{N}, v_{O}$ e $v_{L} \cdot($ Figura 4.6)

Vamos provar que o grafo é 4-conexo. Considere um subconjunto de vértices $X \subseteq V(\mathbb{G}) \backslash\left\{v_{\infty}, v_{S}, v_{N}, v_{O}, v_{L}\right\}$ e observe que na representação retangular existem pelo menos 4 retângulos que envolvem os retângulos correspondentes aos vértices de $X$. Com isso, todo circuito que não define face de $\mathbb{G}$ tem pelo menos 4 arestas. Para concluir que $\mathbb{G}$ é 4-conexo precisamos mostrar que não é possível encontrar um corte de $\mathbb{G}$ com os vértices $v_{N}, v_{S}, v_{L}, v_{O}$ que possua menos que 4 vértices. Assim, para desconectar o grafo devemos remover ao menos dois dos seguintes vértices: $v_{S}, v_{N}, v_{O}, v_{L}$. A figura 4.6 ilustra o fato que devemos remover ao menos dois dos vértices $v_{S}, v_{N}, v_{O}, v_{L}$ para que o grafo resultante não seja conexo. Ainda, note que os vértices a serem removidos para desconectar o grafo devem ser representados por retângulos opostos, por exemplo, $v_{S}$ e $v_{N}$. Caso contrário, precisamos remover ao menos quatro vértices. Perceba que se removermos $v_{S}$ e $v_{N}$, devemos remover um outro vértice que é adjacente a $v_{S}$ e $v_{N}$.

Sem perda de generalidade, suponha que removemos $v_{S}$ e $v_{N}$. Logo, para separar o subconjunto de vértices $A$ de $B$, precisamos remover um outro vértice que é adjacente a $v_{S}$ e $v_{N}$, digamos, $v$. Entretanto, para qualquer vértice de $A$ existe um caminho para qualquer vértice de $B$ que possui o seguinte segmento: $\left\langle v_{O}, v_{\infty}, v_{L}\right\rangle$. Logo, não é possível remover 3 vértices de $\mathbb{G}$ e separar $A$ de $B$.

Portanto, se $\mathbb{G}$ é um grafo plano triangular e possui uma representação retangular cúbica então $\mathbb{G}$ é uma 4-triangulação.

Teorema 4.5: Considere uma representação retangular cúbica $\mathbb{R}$. Existe um grafo planar $\mathbb{G}$ cuja representação retangular cúbica é $\mathbb{R}$ se e só se $\mathbb{G}$ é uma 4-triangulação.

Demonstração: Seja $\mathbb{G}$ o grafo plano triangular do qual $\mathbb{R}$ é uma representação retangular cúbica. Pelo lema 4.4, temos que $\mathbb{G}$ é uma 4-triangulação. 


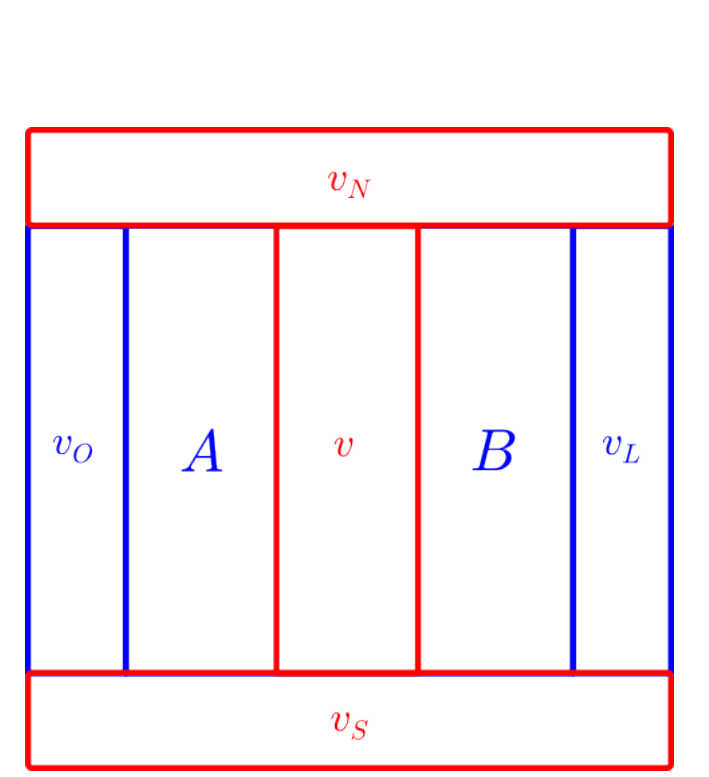

(A)

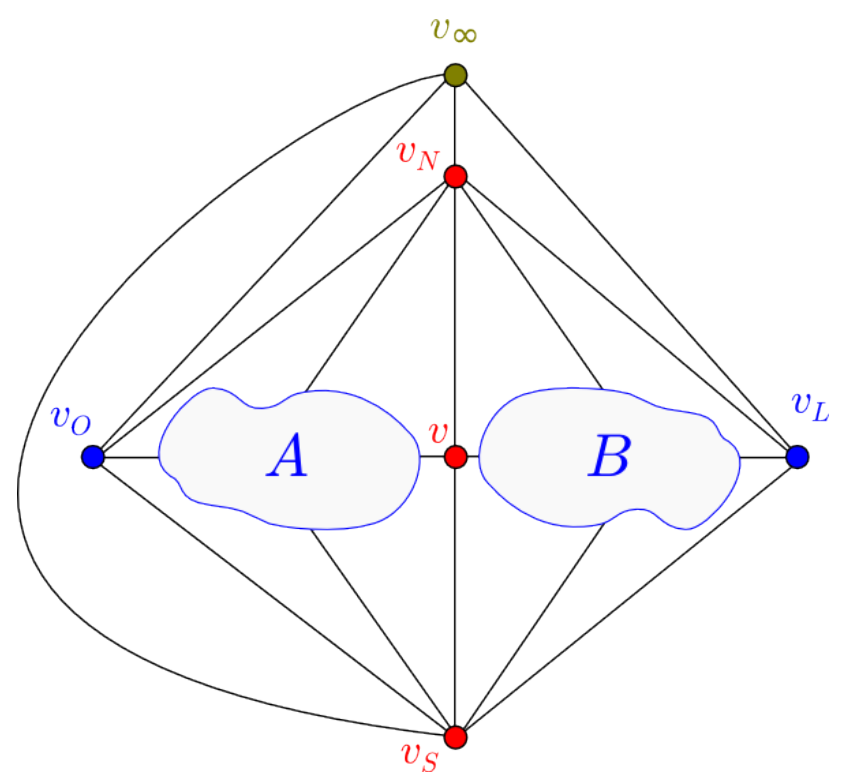

(B)

Figura 4.6: (A) Necessidade da remoção dois vértices do conjunto $\left\{v_{S}, v_{N}, v_{O}, v_{L}\right\}$ para separar $A$ de B. Em vermelho, ilustramos o retângulo que deve ser removido ( $v$ ) caso $v_{S}$ e $v_{N}$ forem removidos. (B) Ilustração dos vértices $v_{S}, v_{N}$ e $v$ em $\mathbb{G}$.

Suponha, por contradição, que a suficiência não vale. Logo, existe ao menos uma 4triangulação de um grafo plano triangulado que não possui uma representação retangular cúbica. Seja $\mathbb{G}$ uma 4-triangulação que não possui uma representação retangular cúbica com uma quantidade mínima de vértices. Seja $v_{\infty}$ um vértice de grau 4 de $\mathbb{G}$ e $v_{L}, v_{N}, v_{O}, v_{S}$ os vértices adjacentes a $v_{\infty}$ em ordem horária (figura 4.7). Pelo lema 4.2, temos que existem duas possibilidades sobre os graus dos vértices $v_{L}, v_{N}, v_{O}$ e $v_{S}$ : (a) ao menos um dos vértices $v_{L}, v_{N}, v_{O}, v_{S}$ possui grau 4; (b) todos os vértices $v_{L}, v_{N}, v_{O}, v_{S}$ possuem grau maior ou igual a 5 .

Considere que um dos vértices $v_{L}, v_{N}, v_{O}, v_{S}$ possui grau 4 . Sem perda de generalidade, suponha que $v_{L}$ possui grau 4 . A figura 4.8 ilustra este caso.

Como existem arestas $v_{L} v_{\infty}, v_{L} v_{N}$, e $v_{L} v_{S}$, temos que a quarta aresta incidente em $v_{L}$ incide em um vértice $v$ diferente de $\left\{v_{L}, v_{N}, v_{O}, v_{S}, v_{\infty}\right\}$. Como ambas as faces que contêm a aresta $v_{L} v$ são triangulares, as arestas $v_{N} v$ e $v_{S} v$ devem existir. Considere o grafo $\mathbb{G}^{\prime}=\mathbb{G}-v_{L}$. Para tornar $\mathbb{G}^{\prime}$ um grafo plano triangular, adicione a aresta $v v_{\infty}$. Precisamos mostrar que o grafo $\mathbb{G}^{\prime}$ é uma 4-triangulação.

Note que $\mathbb{G}$ deve possuir ao menos 7 vértices para contradizer o teorema, pois com 6 vértices existe somente uma 4-triangulação. Assim, $\mathbb{G}^{\prime}$ possui ao menos 6 vértices. Ainda, o vértice $v_{\infty}$ em $\mathbb{G}^{\prime}$ possui grau 4 .

Sejam $x, y \neq r$ dois vértices de $\mathbb{G}$. Como $\mathbb{G}$ é 4-conexo, pelo teorema de Menger, existem 4 caminhos internamente disjuntos entre $x$ e $y$. Considere os caminhos de modo que cada um seja o menor possível. Se nenhum destes caminhos utiliza as arestas $v_{N} v_{L}$ ou $v_{L} v_{S}$, então 


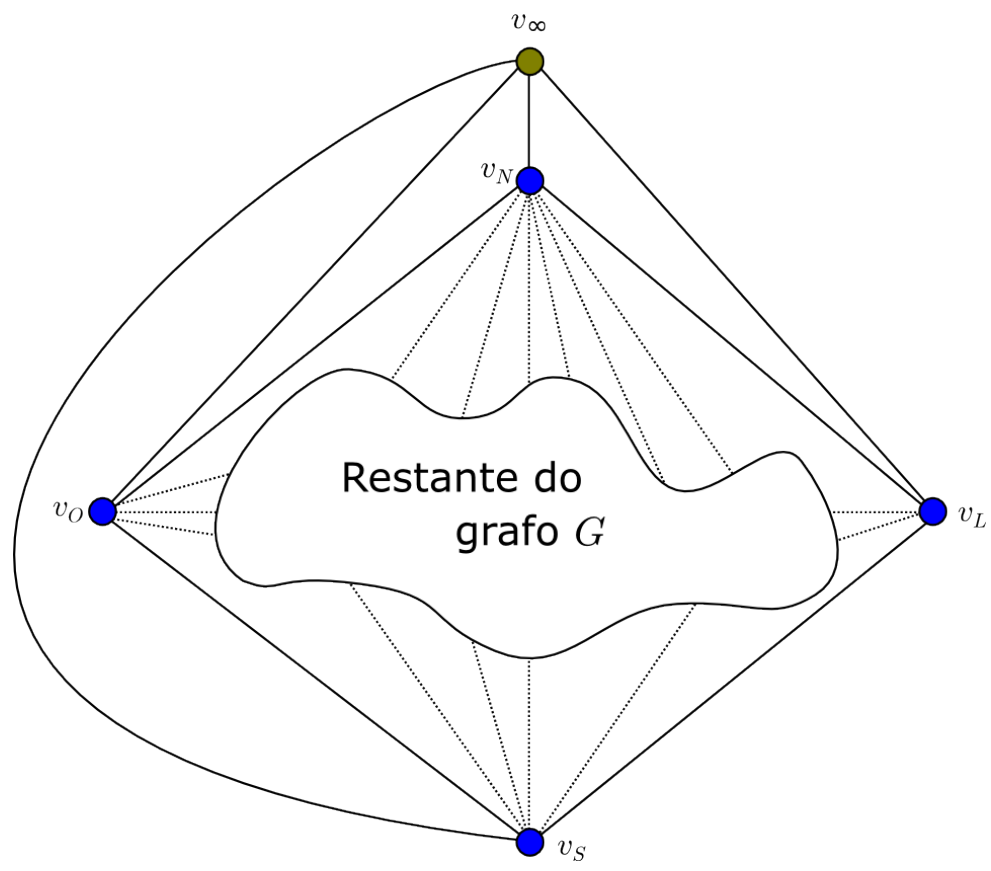

Figura 4.7: Vértices $v_{L}, v_{N}, v_{O}$ e $v_{S}$ de uma 4-triangulação.

estes caminhos também existem em $\mathbb{G}^{\prime}$, ainda, caso exista algum caminho de $\mathbb{G}$ que utilize o segmento $\left\langle v, v_{L}, v_{\infty}\right\rangle$ e não utilize as arestas $v_{N} v_{L}$ e $v_{L} v_{S}$, substitua pela aresta $v v_{\infty}$ em $\mathbb{G}^{\prime}$. Caso contrário, suponha que um dos 4 caminhos utilize a aresta $v_{N} v_{L}$. Pela escolha dos menores caminhos possíveis, este caminho não pode passar por $v_{\infty}$ ou $v$. Assim, o caminho deve utilizar a aresta $v_{L} v_{S}$. Ademais, se um caminho utilizar o vértice $v_{\infty}$, este deve passar por $v_{O}$ e $v_{S}$, e este não será o mais curto possível. Portanto, o vértice $v_{\infty}$ não pertence a nenhum caminho, logo podemos substituir o segmento $\left\langle v_{N}, v_{L}, v_{S}\right\rangle$ de um caminho em $\mathbb{G}$ pelo caminho $\left\langle v_{N}, v_{\infty}, v_{S}\right\rangle$ em $\mathbb{G}^{\prime}$. Concluindo que existem 4 caminhos internamente disjuntos entre quaisquer dois vértices de $\mathbb{G}^{\prime}, \log 0 \mathbb{G}^{\prime}$ é 4-conexo.

Como $\mathbb{G}^{\prime}$ é uma 4-triangulação com menos vértices que $\mathbb{G}$, temos que $\mathbb{G}^{\prime}$ possui uma representação retangular cúbica, pois $\mathbb{G}$ é um contra exemplo com o menor número de vértices. Uma representação retangular cúbica de $\mathbb{G}$ pode ser encontrada a partir da representação retangular cúbica de $\mathbb{G}^{\prime}$. A figura 4.9 ilustra como obter uma representação retangular cúbica de $\mathbb{G}$ a partir da representação retangular cúbica de $\mathbb{G}^{\prime}$. Um absurdo, logo quando um dos vértices $v_{L}, v_{N}, v_{O}, v_{S}$ possui grau 4 a tese segue. 


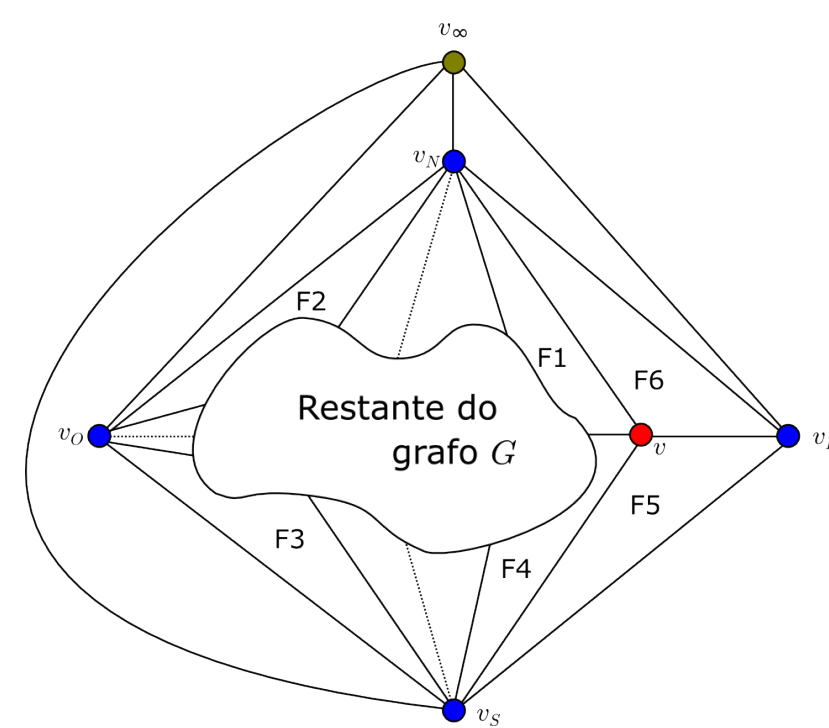

(A)

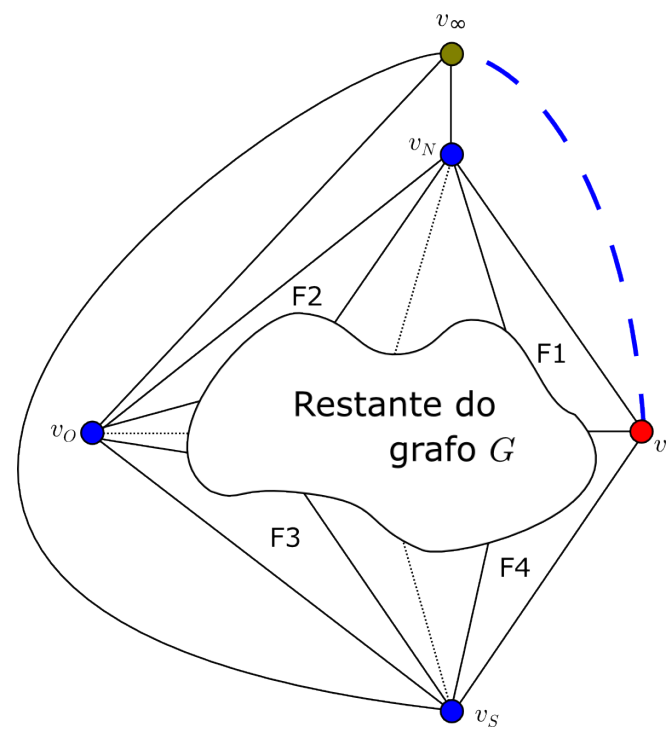

(B)

Figura 4.8: (A)Ilustração de uma 4-triangulação de modo o grau de $v_{L}$ é 4. (B) Ilustração de $\mathbb{G}-v_{L}$ mais a aresta $v v_{\infty}$.

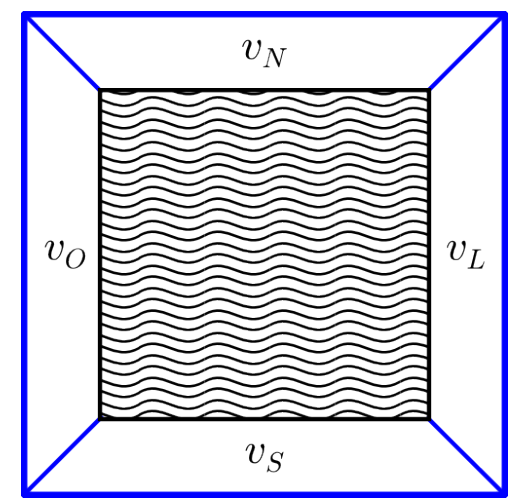

(A)

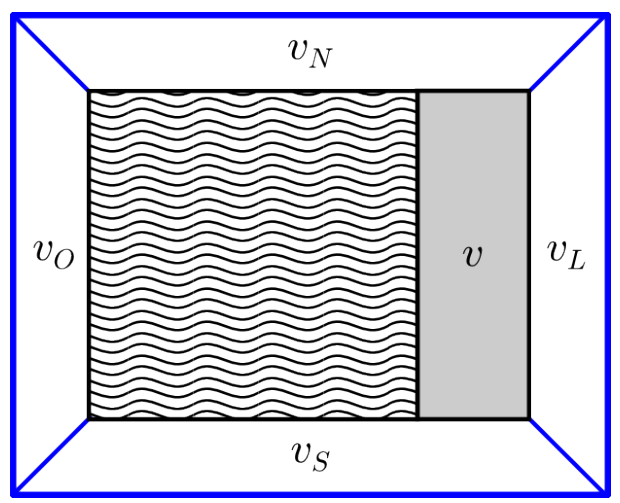

(B)

Figura 4.9: (A) Representação do cubo equivalente a representação retangular cúbica de $\mathbb{G}^{\prime}$. (B) Representação do cubo equivalente a representação retangular cúbica de $\mathbb{G}$ obtida a partir de (A). 
Agora, caso todos os $v_{L}, v_{N}, v_{O}, v_{S}$ possuem pelo menos grau 5. Temos que existem ao menos quatro vértices conforme representados na figura $4.10\left(v_{1}, v_{2}, v_{3}, v_{4}\right)$. A seguir mostraremos que existe um circuito $C$ em $\mathbb{G}$ que passa pelos vértices $v_{L}, v_{\infty}, v_{O}$, mas não passa pelos vértices $v_{S}$ e $v_{N}$. Ainda, $\mathbb{G}-C$ separa $v_{N}$ de $v_{S}$, de modo que $\mathbb{G}-C$ possui exatamente duas componentes conexas com mais de um vértice, onde uma contém o vértice $v_{N}$ e outra contém $v_{S}$. $C$ será chamado de circuito separador.

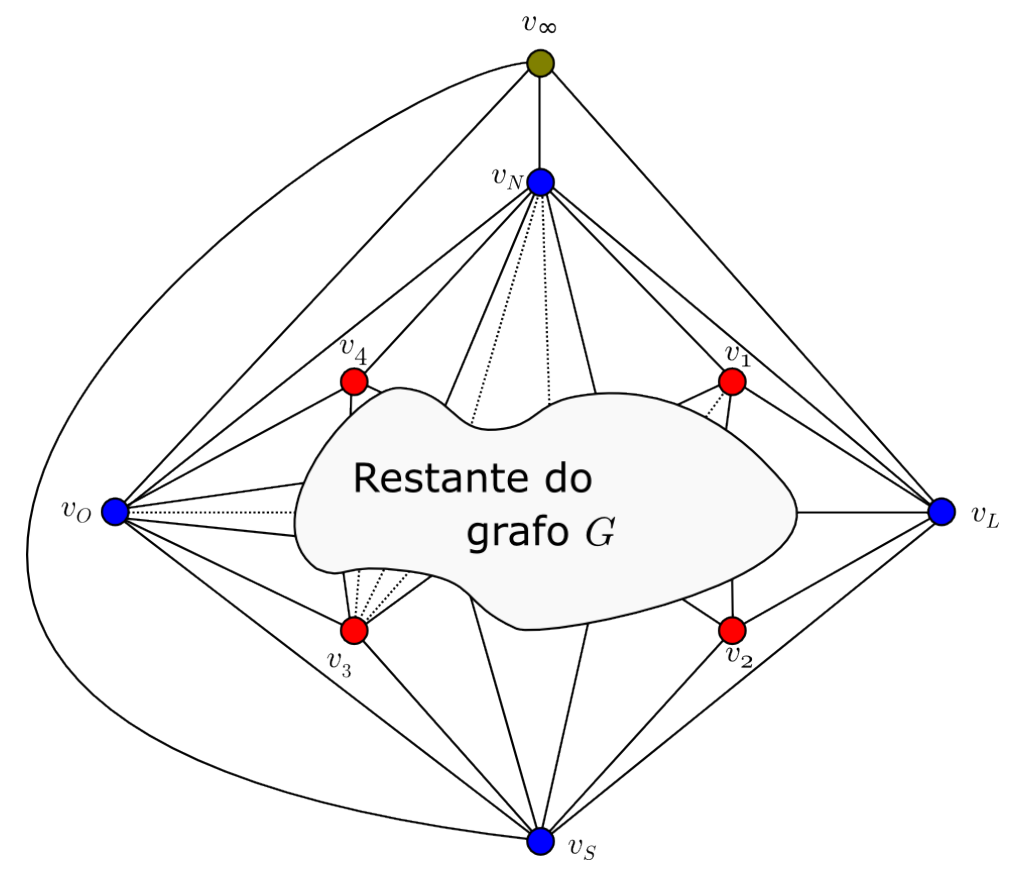

Figura 4.10: Ilustração de uma 4-triangulação onde os vértices $v_{L}, v_{N}, v_{O}, v_{S}$ possuem pelo menos grau 5.[KK85]

Assim, temos dois casos:

Caso 1: Suponha que existe a aresta $v_{1} v_{3}$ em $\mathbb{G}$. Considere $C=\left\langle v_{\infty}, v_{L}, v_{1}, v_{3}, v_{O}, v_{\infty}\right\rangle$. $C$ é um circuito separador que separa $\mathbb{G}$ em duas componentes conexas, de modo que uma contém pelo menos $v_{S}$ e $v_{2}$ e a outra contém ao menos $v_{N}$ e $v_{4}$. A figura 4.11 ilustra este caso.

Caso 2: Suponha que não existe a $\operatorname{aresta} v_{1} v_{3}$ em $\mathbb{G}$. Todos os vértices adjacentes a $v_{S}$ e diferentes de $v_{\infty}, v_{O}$ e $v_{L}$ pertencem ao caminho $\left\langle v_{2}, y_{1}, \ldots, y_{n}, v_{3}\right\rangle$. De modo análogo, todos os vértices adjacentes a $v_{3}$ pertence ao caminho $\left\langle v_{O}, x_{1}, \ldots, x_{m}, v_{S}\right\rangle$. Ademais, estes caminhos possuem um vértice em comum, $y_{n}=x_{m}$. Isso se deve ao fato de que todas as faces de $\mathbb{G}$ são triangulares. A figura 4.12 ilustra estes caminhos.

Nenhum dos vértices $y_{1}, \ldots, y_{n}$ ou $x_{1}, \ldots, x_{n}$ pode ser igual a $v_{N}$ ou $v_{1}$. Caso contrário, implicaria na existência das arestas $v_{S} v_{N}, v_{S} v_{1}$ ou $v_{3} v_{N}$, contradizendo a 4-conexidade de $\mathbb{G}$. Por exemplo, se algum vértice $y_{1}, \ldots, y_{n}$ for igual ao vértice $v_{1}$, logo teríamos a existência da aresta $v_{S} v_{1}$. Assim, teríamos circuito $C=\left\langle v_{L}, v_{S}, v_{1}, v_{L}\right\rangle$ que não é uma face de $\mathbb{G}$. Ainda, $C$ é um corte de $\mathbb{G}$, pois separa $v_{2}$ de $v_{\infty}$. Pela hipótese, $v_{1} v_{3}$ também não existe. Logo, $C=\left\langle v_{\infty}, v_{O}, x_{1}, \ldots, x_{m}, y_{n-1}, \ldots, y_{1}, v_{2}, v_{L}, v_{\infty}\right\rangle$ é um circuito 
separador que separa $\mathbb{G}$ em duas componentes conexas. Uma possui, ao menos, os vértices $v_{S}$ e $v_{1}$ e a outra possui, ao menos, os vértices $v_{N}$ e $v_{3}$.

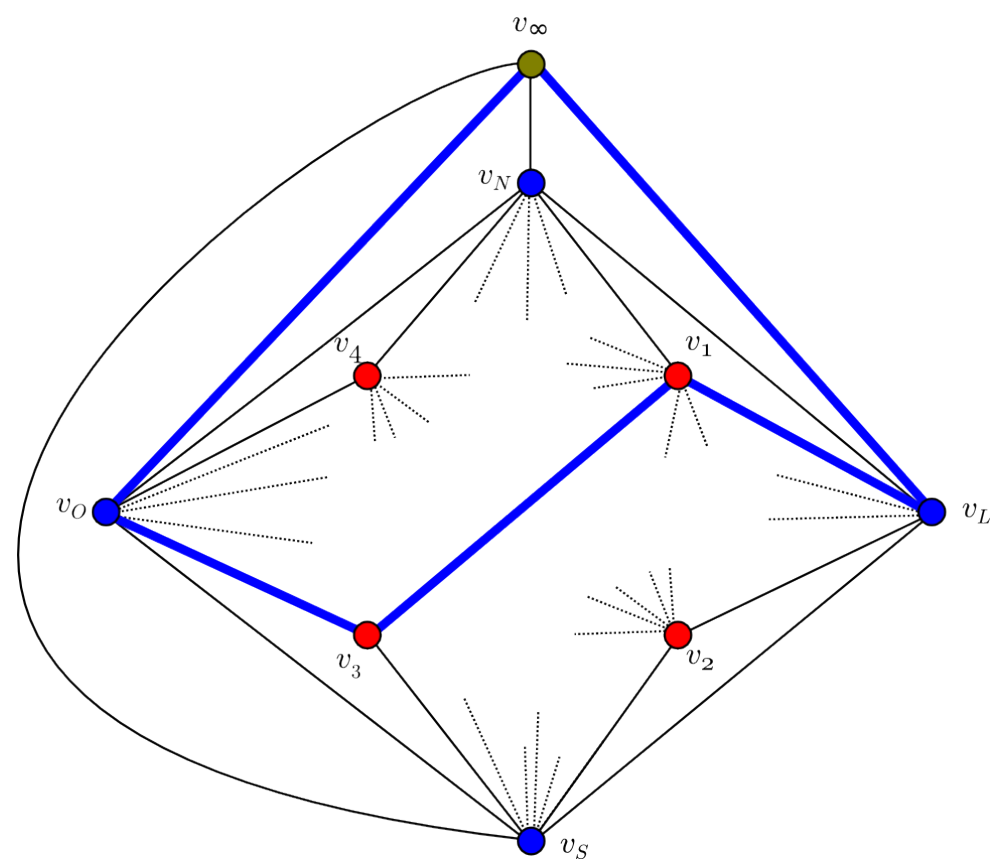

Figura 4.11: Ilustração de um circuito separador quando existe a aresta $v_{1} v_{3}$ em $\mathbb{G}$. As arestas do circuito possui cor azul.

Uma vez que mostramos a existência de um corte separador em $\mathbb{G}$, podemos considerar que $C=\left\langle v_{\infty}, w_{0}, w_{1}, \ldots, w_{n}, w_{n+1}, v_{\infty}\right\rangle$ é o menor circuito separador de $\mathbb{G}$ e que $C$ separa, sem perda de generalidade, $v_{1}$ de $v_{3}$, $\log w_{0}=v_{O}$ e $w_{n+1}=v_{L}$. A figura 4.13 ilustra um circuito $C$, onde $w_{0}=v_{O}$ e $w_{n+1}=v_{L}$.

Seja $\mathbb{G}_{u}=\mathbb{G}_{\text {int }}(C)$ o subgrafo de $\mathbb{G}$ que está no interior de $C$. Adicione a $\mathbb{G}_{u}$ um vértice $v_{S}^{\prime}$ e as arestas $v_{S}^{\prime} v_{\infty}, v_{S}^{\prime} w_{0}, v_{S}^{\prime} w_{1}, \ldots, v_{S}^{\prime} w_{n}, v_{S}^{\prime} w_{n+1}$ conforme ilustrado na figura 4.14. O grafo resultante $\mathbb{G}_{u}$ é um grafo plano triangular. Precisamos mostrar que $\mathbb{G}_{u}$ é uma 4-triangulação.

Como $\mathbb{G}_{u}$ possui os vértices $v_{\infty}, v_{O}, v_{N}, v_{S}^{\prime}$ e $v_{1}$, temos que $\left|V\left(\mathbb{G}_{u}\right)\right| \geq 6$ e o vértice $v_{\infty}$ possui grau 4. Como $\mathbb{G}$ é 4-conexo, temos que cada circuito que não é face e não contém $v_{S}^{\prime}$ possui tamanho maior ou igual a 4 . Precisamos mostrar que todos os circuitos que contém $v_{S}^{\prime}$ que não é face tem tamanho maior que 4.

Todos os circuitos que contém $v_{S}^{\prime}$ e $v_{\infty}$ são os circuitos de três arestas que descrevem uma face de $\mathbb{G}_{u}$. Pois, pela construção de $\mathbb{G}_{u}$ não existe a aresta $v_{S}^{\prime} v_{N}$ e pela 4 -conexidade de $\mathbb{G}$ não existe a aresta $v_{O} v_{L}$. Para qualquer outro circuito que contenha $v_{S}^{\prime}$, possui tamanho igual a 3 é da forma $\left\langle w_{i}, w_{j}, v_{S}^{\prime}, w_{i}\right\rangle$, onde $i=j \pm 1$. Caso contrário $(i \neq j \pm 1)$, a existência da aresta $w_{i} w_{j}$ iria implicar que $C$ não é o menor circuito separador de $\mathbb{G}$. Assim, todo circuito que não é face em $\mathbb{G}_{u}$ possui tamanho maior ou igual a 4 , e consequentemente $\mathbb{G}_{u}$ é 4-conexo. Portanto, $\mathbb{G}_{u}$ é uma 4-triangulação.

Podemos construir $\mathbb{G}_{b}$ de forma análoga a $\mathbb{G}_{u}$. Assim, podemos concluir que $\mathbb{G}_{b}$ também 


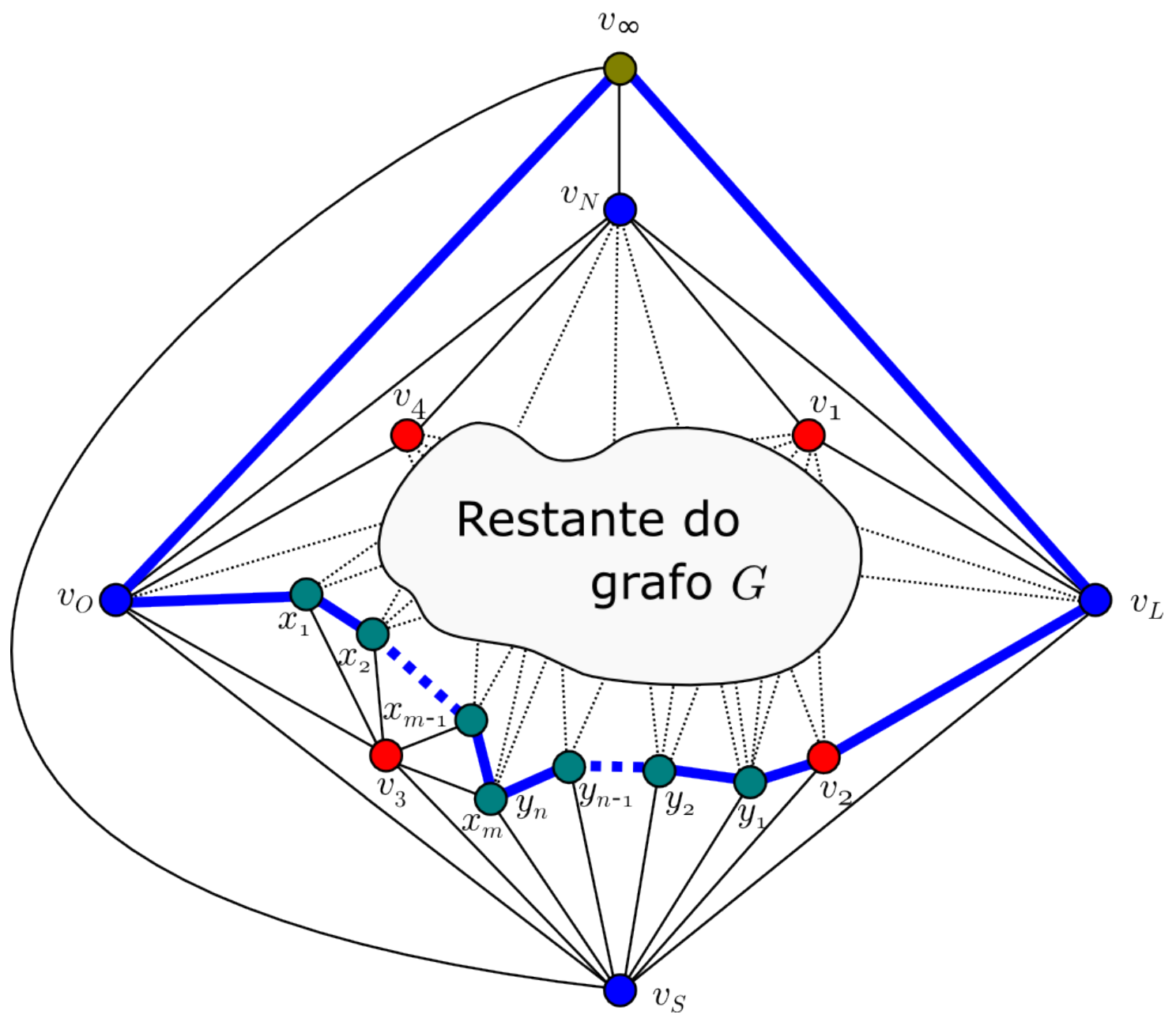

Figura 4.12: Ilustração de um circuito separador quando não existe a aresta $v_{1} v_{3}$ em $\mathbb{G}$. As arestas do circuito possui cor azul.

é uma 4-triangulação.

Observe que todas as faces de $\mathbb{G}_{u}$ e $\mathbb{G}_{b}$ são triangulares e que $\left|V\left(\mathbb{G}_{u}\right)\right|,\left|V\left(\mathbb{G}_{b}\right)\right|<|V(\mathbb{G})|$. Pela hipótese, $\mathbb{G}$ é o grafo com a menor quantidade de vértices que não possui uma representação retangular cúbica, logo $\mathbb{G}_{u}$ e $\mathbb{G}_{b}$ possuem representações retangulares cúbicas. Podemos colocar um cubo em cima do outro (possivelmente após rotacionar algum cubo) de modo que vamos unir as faces que possuem um sistema de subdivisão retangular nos dois cubos a fim de tornar a face que irá possuir um sistema de subdivisão retangular da união dos cubos. Ainda, o cubo resultante deve ser uma representação retangular cúbica do grafo G. A figura 4.15 ilustra esta união.

Portanto, temos um absurdo. Pois, não existe uma 4-triangulação que não possui uma representação retangular cúbica. 


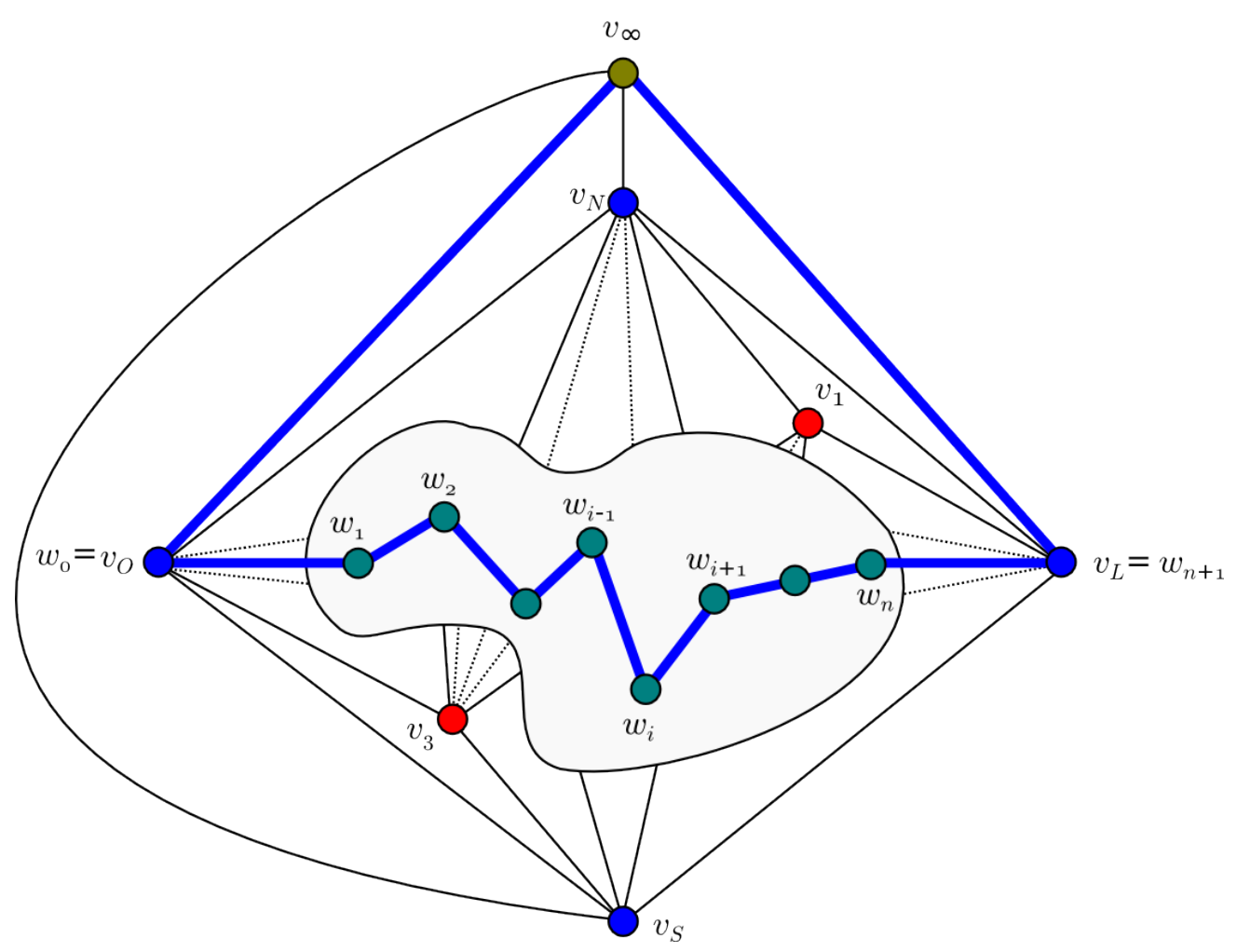

Figura 4.13: Ilustração de um circuito separador $C$ em $\mathbb{G}$, onde $w_{0}=v_{O}$ e $w_{n+1}=v_{L}$. [KK85]

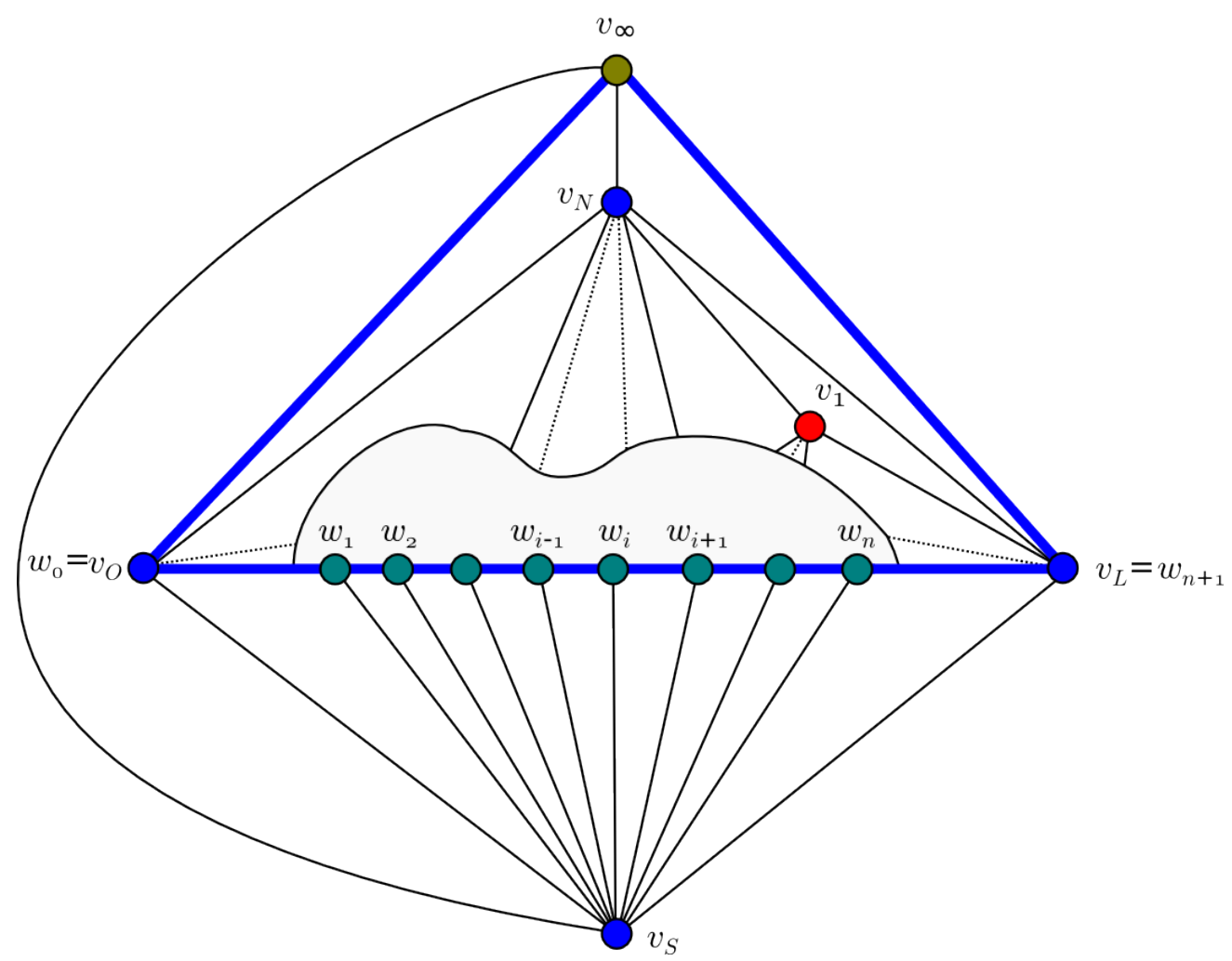

Figura 4.14: Construção do grafo $\mathbb{G}_{u}$ a partir do circuito separador C. [KK85] 


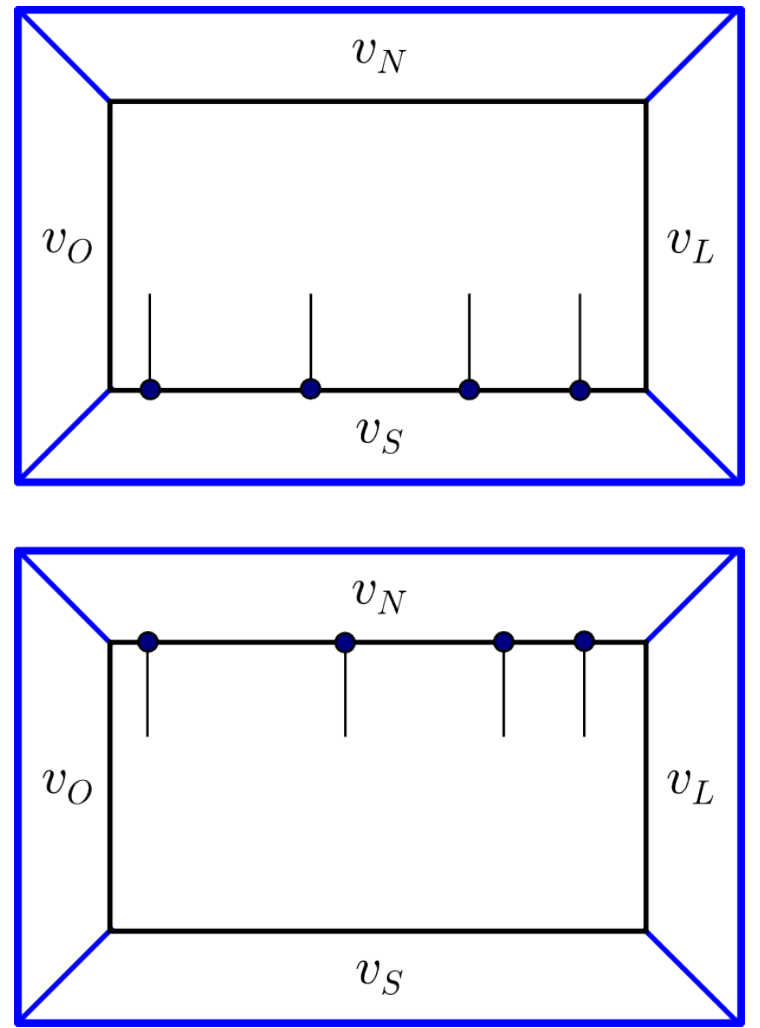

(A)

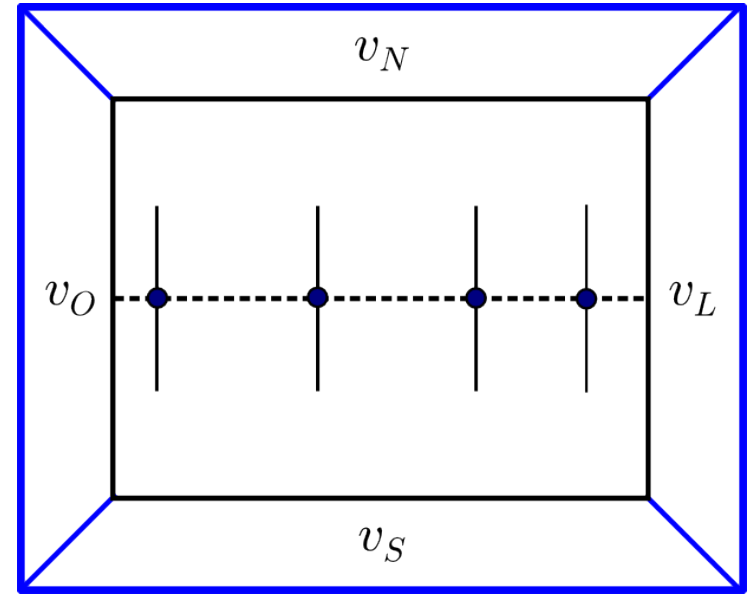

(B)

Figura 4.15: União dos cubos duais aos grafos $\mathbb{G}_{b} e \mathbb{G}_{u}$ de modo que o cubo resultante é uma representação retangular cúbica do grafo $\mathbb{G}$. [KK85]

Como fazer agora para usar a caracterização e resolver o problema de representação retangular? Até o momento apresentamos resultados que caracterizam a existência de uma representação retangular cúbica. A partir de agora, nosso interesse é encontrar uma representação retangular de um grafo plano triangulado.

A seguir, apresentamos um corolário do teorema 4.5.

Teorema 4.6: Um grafo plano triangulado $\mathbb{G}$ possui uma representação retangular se e somente se existe uma 4-triangulação $\mathbb{H}$ de modo que é possivel obter $\mathbb{G}$ a partir da remoção do vértice de grau $4\left(v_{\infty}\right)$ e seus vizinhos em $\mathbb{H}$.

A seguir, vamos mostrar quais condições são necessárias para que um grafo $\mathbb{G}$ seja subgrafo do grafo $\mathbb{H}$ definido no teorema 4.6. Se existir tal grafo $\mathbb{H}$, vamos denominá-lo de 4-extensão (4-completion) de $\mathbb{G}$.

Uma forma trivial para verificar se existe uma 4-extensão de um grafo $\mathbb{G}$ é tentar adicionar os vértices $v_{L}, v_{N}, v_{O}, v_{S}$ e $v_{\infty}$ que estão faltando. A figura 4.7 ilustra os vértices que estão faltando e são necessários para a existência de uma 4-extensão de $\mathbb{G}$. Note que como $\mathbb{G}$ é interno ao circuito $\left\langle v_{L}, v_{N}, v_{O}, v_{S}, v_{L}\right\rangle$, precisamos adicionar algumas arestas entre os vértices 
do circuito e vértices que pertencem à face externa de $\mathbb{G}$. Precisamos garantir que a adição das novas arestas a $\mathbb{G}$ deixe o grafo plano resultante triangular e 4-conexo.

Vamos mostrar a construção para grafos 2-conexos e depois iremos estendê-la para grafos gerais.

Dizemos que um grafo é um bloco se não existe um vértice que é articulação, ou seja, se o grafo é 2-conexo. A figura 4.16 ilustra um grafo 2-conexo e um grafo que não é 2-conexo.

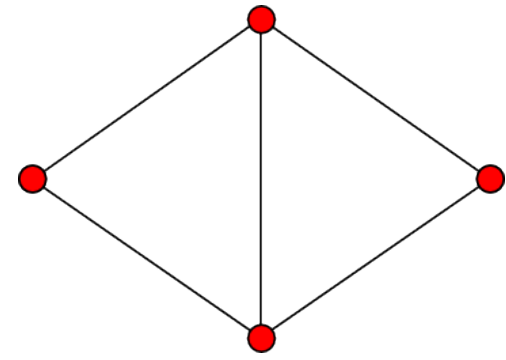

(A) Grafo 2-conexo (bloco)

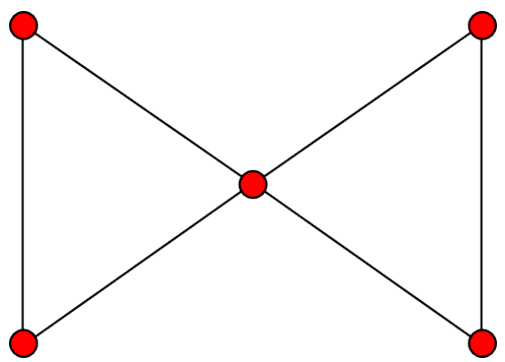

(B) Grafo que não é 2-conexo

Figura 4.16: Exemplo de grafo que é um bloco e outro que possui um vértice de articulação.

A seguir, vamos mostrar alguns resultados para um grafo que é um bloco. Futuramente, vamos estender para grafos gerais.

\subsection{ConstruçÃo de uma 4-EXtensão PARA UM BLOCO}

Considere que $\mathbb{G}$ é um grafo plano triangulado 2-conexo. Como apresentado anteriormente, para construir uma 4-extensão de um bloco precisamos incluir em $\mathbb{G}$ cinco vértices e definir onde quatro destes devem se conectar a $\mathbb{G}$. Note que só podemos conectar estes vértices nos vértices que pertencem ao circuito mais externo de $\mathbb{G}$. Isso é equivalente a escolher os cantos de uma representação retangular de $\mathbb{G}$, caso exista uma representação retangular cúbica da 4-extensão de $\mathbb{G}$.

Para definir os cantos, vamos associar quatro rótulos $\left(v_{S E}, v_{S O}, v_{N O}, v_{N E}\right)$ a um conjunto de, no máximo, quatro vértices de $\mathbb{G}$ que incidem na face externa. Estes vértices dividem o circuito que define a face externa de $\mathbb{G}$ em, no máximo, quatro caminhos disjuntos nas arestas. Depois de definir estes caminhos, vamos conectar cada um dos vértices incidentes à face externa de $\mathbb{G}$ a um dos vértices: $v_{S}, v_{L}, v_{O}$ e $v_{N}$. Para todos os vértices entre os vértices $v_{N O}$ e $v_{N E}$, incluindo $v_{N O}$ e $v_{N E}$, vamos conectá-los a $v_{N}$. Para todos os vértices entre os vértices $v_{N E}$ e $v_{S E}$, incluindo $v_{N E}$ e $v_{S E}$, vamos conectá-los a $v_{L}$, e assim por diante.

Se o grafo resultante a partir da associação dos vértices $v_{S E}, v_{S O}, v_{N O}$ e $v_{N E}$ implicar em uma 4-extensão, então existe uma representação retangular cúbica da 4-extensão encontrada, a partir da qual obtemos uma representação retangular de $\mathbb{G}$. A figura 4.17A ilustra um 
exemplo de um bloco e uma atribuição dos rótulos $v_{S E}, v_{S O}, v_{N O}$ e $v_{N E}$. Os caminhos externos são: $\left\langle v_{S E}, v_{5}, v_{S O}\right\rangle,\left\langle v_{S O}\right\rangle,\left\langle v_{N O}, v_{1}, v_{N E}\right\rangle$ e $\left\langle v_{N E}, v_{4}, v_{S E}\right\rangle$.

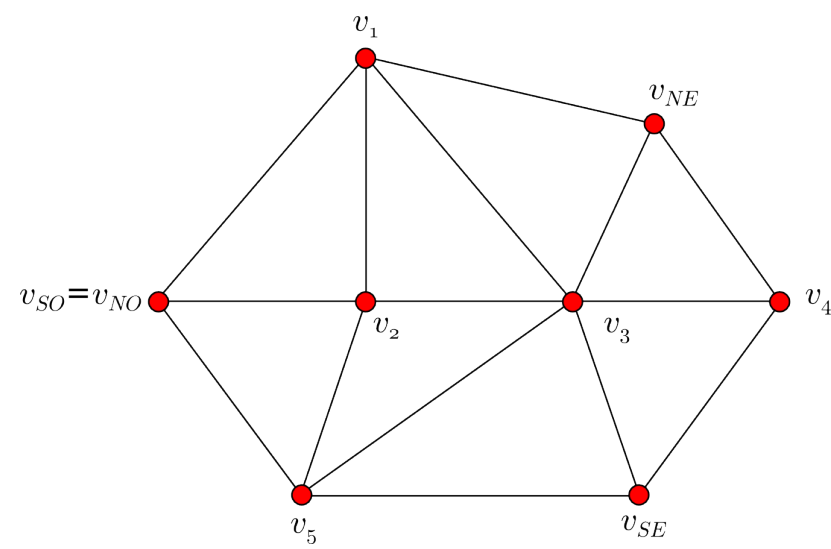

(A) Bloco $\mathbb{G}$ com os rótulos $v_{S E}, v_{S O}, v_{N O}$ e $v_{N E}$ definidos.

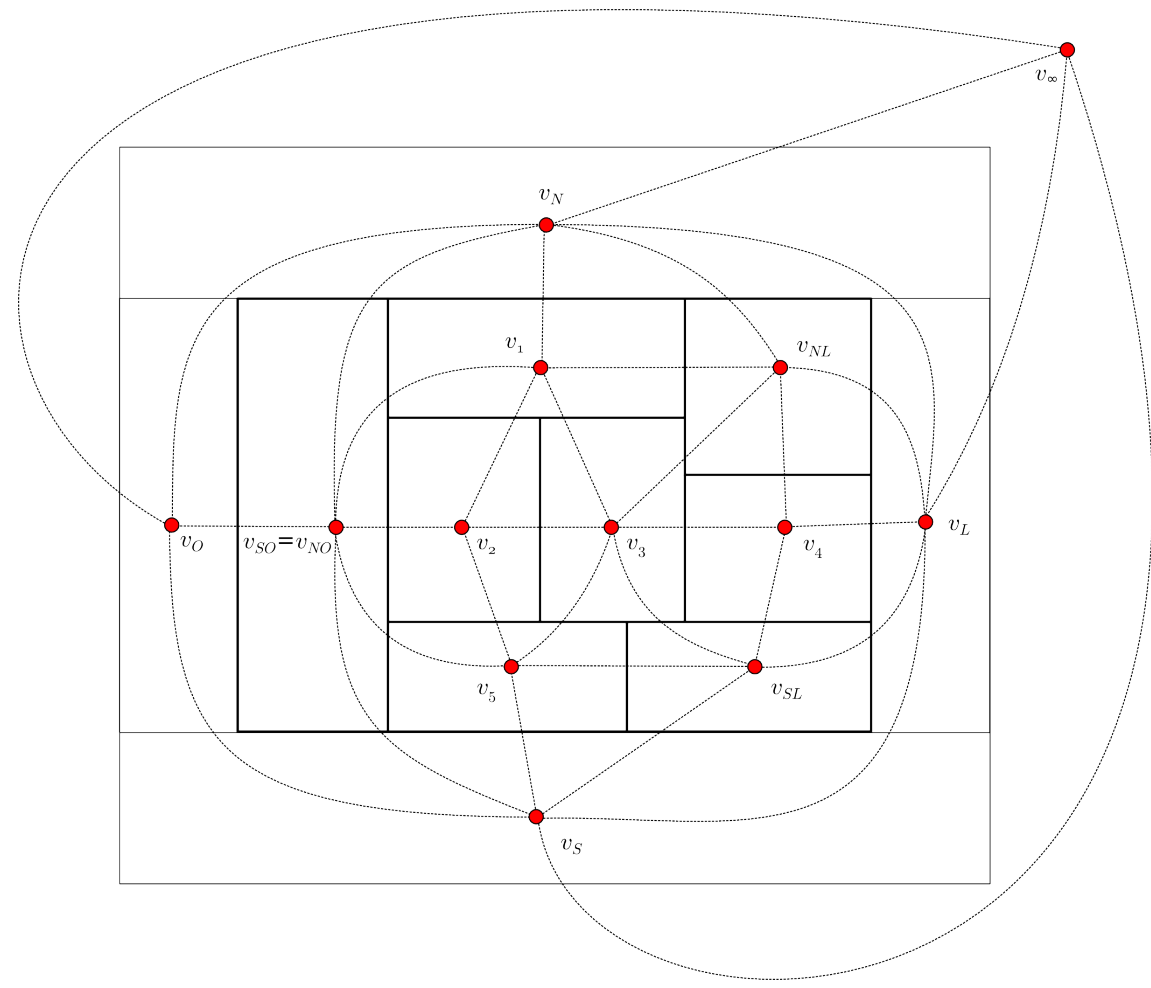

(B) 4-extensão de $\mathbb{G}$.

Figura 4.17: Exemplo de um bloco $\mathbb{G}$ e a representação retangular cúbica da 4-extensão de $\mathbb{G}$. [KK85] 
Os rótulos não precisam ser associados a 4 vértices distintos. Se algum vértice $v$ for associado a dois rótulos consecutivos, por exemplo, $v_{S O}$ e $v_{N O}$, então a face que corresponde ao vértice $v$ irá possuir dois cantos do retângulo mais externo da representação retangular. Na figura $4.17 \mathrm{~A}$ os rótulos $v_{N O}$ e $v_{S O}$ foram associados a um mesmo vértice. Note que a face correspondente a $v_{N O}=v_{S O}$ em $4.17 \mathrm{~B}$ contém dois cantos.

Ainda, nenhum vértice pode possuir mais que dois rótulos. Salvo no caso trivial, onde só existe um vértice e este possui os quatro rótulos. (Figura 4.21)

Podem existir diversas formas de atribuir os rótulos aos vértices de $\mathbb{G}$ de modo que exista uma 4-extensão. Note que a quantidade não é maior que $\left(\begin{array}{l}x \\ 4\end{array}\right)+3\left(\begin{array}{l}x \\ 3\end{array}\right)+\left(\begin{array}{l}x \\ 2\end{array}\right)=\frac{x(x-1)\left(x^{2}+7 x-6\right)}{24}$ onde $x \geq 2$ é o número de vértices da face externa de $\mathbb{G}$. Esta quantidade é referente à atribuição dos quatro rótulos de modo que nenhum vértice receba mais do que dois rótulos.

Entretanto, não é necessário examinar todas as escolhas de associações dos rótulos para determinar se existe uma 4-extensão de $\mathbb{G}$. O processo de construção da 4-extensão falha se criarmos um circuito contendo menos que 4 arestas e que não é face, ou se tal circuito já existir em $\mathbb{G}$ (lema 4.3 e teorema 4.5). Se o circuito já existir, então o grafo não possui uma representação retangular independentemente da escolha dos rótulos. Caso contrário, vamos apresentar uma propriedade que elimina algumas associações.

Seja $C_{0}(\mathbb{G})$ o circuito da face externa de $\mathbb{G}$. Uma corda de $C_{0}(\mathbb{G})$ é uma aresta $\alpha$ que é adjacente a dois vértices de $C_{0}(\mathbb{G})$, porém $\alpha \notin E\left(C_{0}(\mathbb{G})\right)$. Sempre que nos referirmos a uma corda de $\mathbb{G}$, estamos nos referindo a uma corda do circuito mais externo de $\mathbb{G}$.

Qualquer circuito criado com a adição de arestas em $\mathbb{G}$ da forma descrita acima contém ao menos um dos vértices $v_{N}, v_{L}, v_{S}, v_{O}$ e tamanho maior ou igual a 3 . Pela forma que completamos o grafo $\mathbb{G}$, todos os circuitos que contêm $v_{\infty}$ e não formam uma face possuem tamanho maior que 3. Um circuito que contém 3 arestas que não é face só é criado se um dos vértices $v_{N}, v_{L}, v_{S}, v_{O}$ é adjacente a dois vértices da face externa de $\mathbb{G}$ e existe uma corda entre eles. A existência de tal circuito, pelo teorema 4.5 implicaria que a extensão de $\mathbb{G}$ não seria 4-conexo. Assim, tais circuitos devem ser evitados.para evitar estes tipos de circuitos, os rótulos $v_{S E}, v_{S O}, v_{N O}$ e $v_{N E}$ serão atribuídos de modo não existe um vértice do conjunto $\left\{v_{N}, v_{L}, v_{S}, v_{O}\right\}$ que seja adjacente a ambos extremos de uma corda.

Conforme ilustrado na figura $4.18, C_{0}(\mathbb{G})$ pode ter muitas cordas. Isso implica que precisamos definir os rótulos $v_{N}, v_{L}, v_{S}, v_{O}$ de modo que quando adicionarmos os vértices $v_{S}, v_{N}, v_{L}$ e $v_{O}$, os extremos de qualquer corda não pertençam a um triângulo que não é face. Como já argumentado anteriormente, para que os extremos $u, v$ de uma corda não formem um triângulo que não é face, precisamos escolher ao menos um vértice interno do caminho de $u$ a $v$ que utiliza somente arestas de $C_{0}$. É fácil ver que se rotularmos um dos extremos deste caminho teremos um triângulo que não é face. 
Nem todas as cordas de $C_{0}(\mathbb{G})$ precisam ser analisadas para satisfazer estas restrições. Note que as cordas podem possuir outras cordas internas a ela. Uma corda com extremos $u$ e $v$ é chamada de corda crítica se nenhum dos vértices internos do menor caminho de $u$ a $v$ que utiliza somente arestas de $C_{0}(\mathbb{G})$ contém um extremo de uma outra corda, este caminho será conhecido como caminho crítico. Deste modo, para satisfazer as restrições de todas as cordas de $C_{0}(\mathbb{G})$, precisamos satisfazer somente as restrição para os extremos das cordas críticas de $C_{0}(\mathbb{G})$.

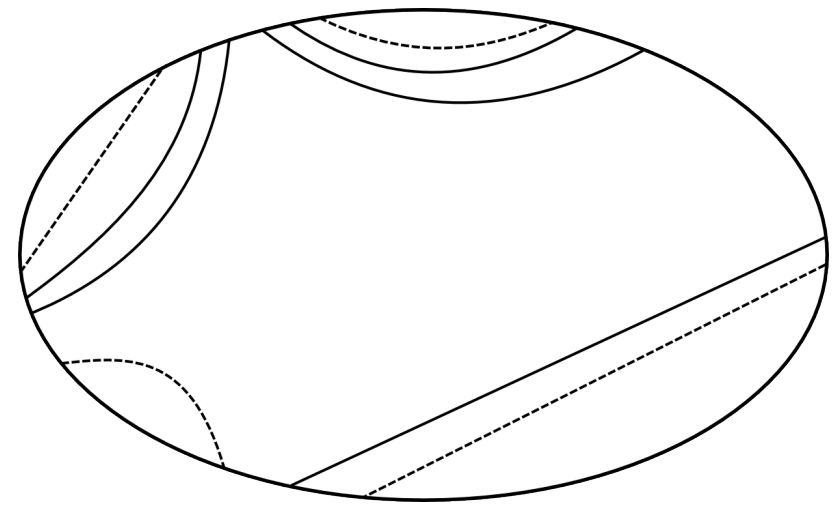

Figura 4.18: Exemplo de uma configuração das cordas do circuito da face externa. As cordas críticas são representadas por uma linha pontilhada.

Teorema 4.7: Um grafo plano triangulado $\mathbb{G}$ que é 2-conexo, em que cada circuito que não é face possui tamanho maior ou igual a 4 possui uma representação retangular se e somente se não existem mais que 4 caminhos críticos.

Demonstração: Suponha que existe uma representação retangular de $\mathbb{G}$. Se $\mathbb{G}$ possuir 5 ou mais caminhos críticos, então para qualquer escolha dos vértices da face externa de $\mathbb{G}$ para os rótulos $v_{S E}, v_{S O}, v_{N O}$ e $v_{N E}$, temos que ao menos um dos caminhos, por exemplo, $v_{S O}$ a $v_{S E}$, irá conter ao menos 3 extremos de cordas críticas, onde 2 destes são extremos de uma determinada corda crítica. Consequentemente, a construção da 4-extensão irá falhar, pois existirá um triângulo que desconecta ao menos um vértice do restante do grafo. Um absurdo com o fato de $\mathbb{G}$ possuir uma representação retangular.

Suponha agora que $\mathbb{G}$ possui $\eta \leq 4$ caminhos críticos. Precisamos encontrar uma rotulação dos vértices da face externa de $\mathbb{G}$ de modo que exista uma representação retangular. Ainda, note que existe uma ordem para os rótulos, precisamos rotular os vértices da face externa de modo que quando percorrermos o circuito da face externa no sentido horário os rótulos $v_{S E}, v_{S O}, v_{N O}$ e $v_{N E}$ apareçam nesta ordem.

Para cada caminho crítico, escolha um de seus vértices internos para rotulá-lo apropriadamente. Para os $4-\eta$ rótulos restantes escolha um vértice qualquer da face externa.

Deste modo, todos os circuitos que não são faces possuem ao menos 4 arestas. Portanto, existe uma representação retangular de $\mathbb{G}$. 


\subsection{ConstruÇÃo de uma 4-EXtensão para grafos GERAIS}

Nesta seção, vamos apresentar uma construção para grafos planos conexos. Um grafo plano conexo, não precisa ser, necessariamente, um bloco, ele pode conter um conjunto de blocos maximais, de modo que, dois blocos maximais contêm, no máximo, um vértice em comum, perceba que este vértice é uma articulação.

Seja $\mathbb{G}$ um grafo plano triangulado. Considere que $B_{1}, B_{2}, \ldots, B_{k}$ são blocos maximais de $\mathbb{G}$. Como todas as faces de $\mathbb{G}$ são triângulos, não é possível desenhar um bloco maximal $B_{j}$ dentro de uma face de um bloco maximal $B_{i}$. Pela figura 4.19 vemos que não é possível desenharmos um bloco maximal em uma face de um outro bloco maximal, pois, ou contradizemos que todas as faces de $\mathbb{G}$ sejam triângulos, ou com o fato de $B_{i}$ e $B_{j}$ serem blocos maximais. Na figura 4.19 os vértices de um bloco maximal é representado pela cor azul enquanto os vértices de um outro bloco maximal possuem cor vermelha. Se o bloco com os vértices vermelhos for desenhado na face do bloco dos vértices azuis, então deve existir mais uma aresta entre algum vértice vermelho a um vértice azul pois todas as faces do grafo possuem três arestas. Entretanto, caso exista tal aresta, contrariamos o fato de que $B_{i}$ e $B_{j}$ são blocos maximais.

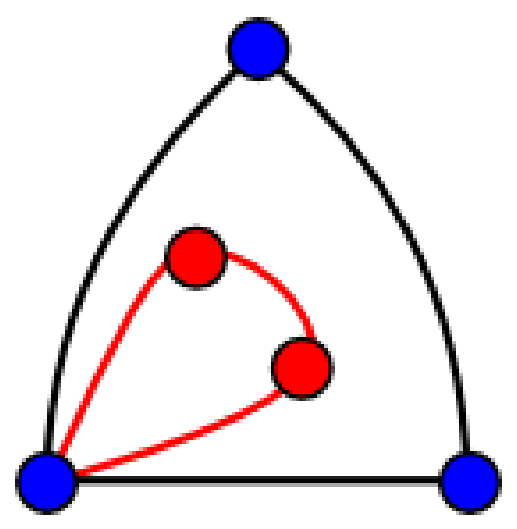

Figura 4.19: Ilustração do bloco maximal $B_{j}$ (vértices vermelhos) desenhado em uma face do bloco maximal $B_{i}$ (vértices azuis). O circuito externo de $B_{j}$ possui cor vermelha.

É fácil ver que se $\mathbb{G}$ é um grafo plano triangulado, então todas as articulações de $\mathbb{G}$ pertencem à face externa de $\mathbb{G}$. Suponha que existe um vértice $v$ que é uma articulação $\mathbb{G}$ de modo que $v$ não pertence a face externa. Sejam $\left\{v_{1}, v_{2}, \ldots, v_{k}\right\}$ os vizinhos de $v$ em ordem horária. Como $v$ não pertence a face externa e como todas as faces internas são triangulares, então há o circuito $\left\langle v_{1}, v_{2}, \ldots, v_{n}, v_{1}\right\rangle$ em $\mathbb{G}$.

Logo, podemos encontrar todas as articulações percorrendo o ciclo da face externa de $\mathbb{G}$. A ideia é encontrar todas articulações para obter todos os blocos maximais de $\mathbb{G}$.

Definimos o grafo de vizinhança dos blocos como um grafo onde cada vértice representa 
um bloco maximal de $\mathbb{G}$, e existe uma aresta se os respectivos blocos maximais possuem um vértice em comum.

Teorema 4.8: Um grafo plano triangulado $\mathbb{G}$ possui uma representação retangular se e somente se o grafo de vizinhança dos blocos é um caminho.

Podemos interpretar o teorema 4.8 da seguinte forma: Para cada bloco maximal, encontramos sua respectiva representação retangular. Após encontrar todas as representações retangulares, podemos juntá-las de modo que a representação retangular resultante seja a representação retangular de $\mathbb{G}$. Esta junção equivale a satisfazer as restrições impostas pelo grafo de vizinhança dos blocos. A figura 4.20 mostra um exemplo de um grafo de vizinhança dos blocos de 4 vértices que não é um caminho. Perceba que é impossível desenhar uma representação retangular, pois como não existem arestas entre os blocos maximais $a$ e $d$ e entre os blocos $c$ e $d$, logo, temos que o desenho resultante não é um retângulo.

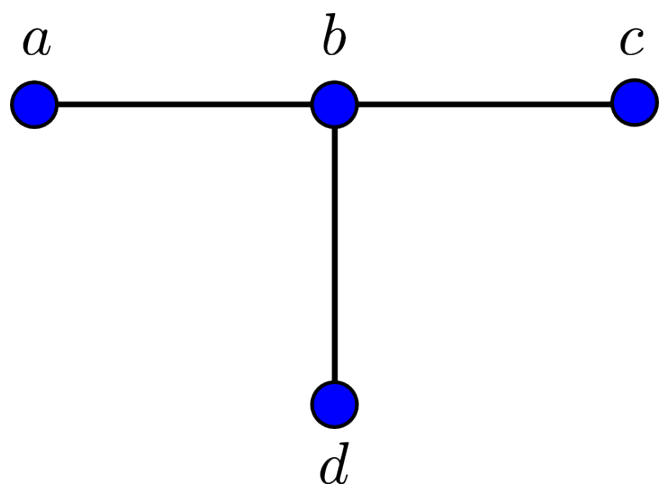

(A)

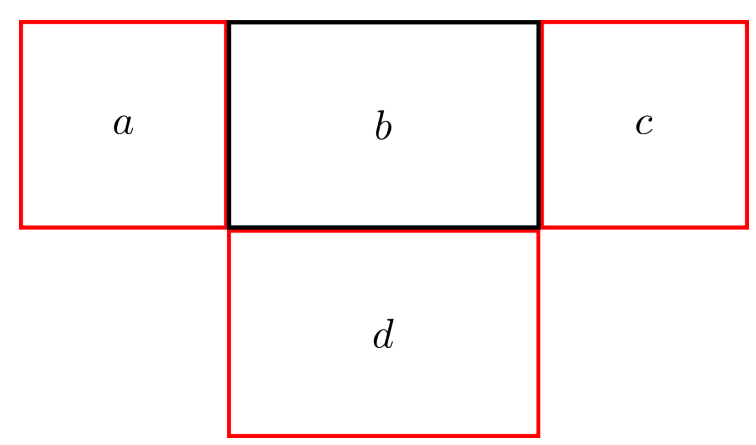

(B)

Figura 4.20: (A) Grafo de vizinhança dos blocos com 4 vértices. (B) Um desenho das restrições impostas por $(A)$

Um caminho crítico em um bloco maximal $B_{i}$ é um caminho crítico no subgrafo $B_{i}$ que não contém nenhuma articulação de $\mathbb{G}$.

Teorema 4.9: Um grafo plano triangulado $\mathbb{G}$ possui uma representação retangular se e somente se (1) cada circuito que não é face possui ao tamanho maior ou igual a 4, (2) o grafo da vizinhança dos blocos é um caminho, (3) ambos os blocos maximais correspondentes aos vértices dos extremos do caminho do grafo da vizinhança dos blocos devem conter, cada um, no máximo 2 caminhos críticos, e (4) nenhum outro bloco maximal contém um caminho crítico. 
Este último teorema é a consequência do fato de que as articulações de $\mathbb{G}$ correspondem às faces retangulares da representação retangular que possui arestas em dois lados opostos do retângulo mais externo da representação retangular. Ainda, é fácil ver que para existir uma representação retangular cada bloco maximal $B_{i}$ deve possuir uma representação retangular para que $\mathbb{G}$ também a tenha.

\subsection{Algoritmo de Koźmiński E Kinnen PARA ENCONTRAR UMA REPRESENTAÇÃO RETANGULAR}

Nesta seção, vamos apresentar o algoritmo que foi desenvolvido por Koźmiński e Kinnen [KK84] que implementa a caracterização apresentada neste capítulo.

O algoritmo que será apresentado recebe como entrada um grafo plano triangulado $\mathbb{G}$ e deve devolver uma representação retangular de $\mathbb{G}$. Caso não exista tal representação é devolvido um certificado da inexistência. As três primeiras etapas do algoritmo consistem em fazer verificações em tempo linear. Caso o algoritmo falhe em alguma destas etapas, não existe uma forma de construir uma 4-extensão de $\mathbb{G}$. Para facilitar a leitura do algoritmo, quando ocorrer uma falha, subentende-se que além da falha, o certificado também é devolvido pelo algoritmo.

A representação retangular de $\mathbb{G}$ que o algoritmo devolve, consiste em determinar para cada aresta de $\mathbb{G}$ se está será horizontal ou vertical, pois, como já dito anteriormente, existe uma bijeção entre as arestas de $\mathbb{G}$ e das arestas que não incidem na face externa da representação retangular.

\section{Algoritmo KoŹmińsKi E Kinnen}

ENTRADA: Grafo plano triangulado $\mathbb{G}$.

SAÍDA: Representação retangular de $\mathbb{G}$.

1. Verifique se todos os vértices que não pertencem à face externa possuem grau maior ou igual a 4 e se todas as faces, com exceção da face externa, possuem 3 arestas;

2. Encontre todos os blocos maximais de $\mathbb{G}$ utilizando uma busca em profundidade. Verifique se o grafo da vizinhança dos blocos é um caminho;

3. Identifique as cordas e caminhos críticos que implicam em cantos de $\mathbb{G}$. Verifique se existem mais que 4 caminhos críticos devolva falha. Ainda, caso $\mathbb{G}$ não seja um bloco, verifique se os caminhos críticos ocorrem somente nos blocos que são os vértices extremos do grafo da vizinhança dos blocos e que estes ocorrem no máximo 2 vezes; 
4. Escolha um vértice pertencente a cada um dos caminhos que implicam em cantos, uma vez que este vértice não pode ser um extremo do caminho. Se foram escolhidos menos que 4 vértices, selecione $4-\eta$ vértices da face externa de $\mathbb{G}$, onde $\eta$ é o número de caminhos críticos que implicam em cantos. Assim, $\mathbb{G}$ foi dividido em 4 caminhos disjuntos nas arestas. Os vértices escolhidos serão os cantos da representação retangular. Construa uma 4-extensão $\mathbb{H}$ de $\mathbb{G}$ conforme ilustrado na figura 4.17B;

5. Neste passo, iremos determinar as orientações das arestas da representação retangular de $\mathbb{G}$. Este passo divide a 4-extensão de $\mathbb{G}$ em 4-extensões menores. Este passo é equivalente a dividir a representação retangular em representações retangulares menores, ou seja, com menos faces, para isso, execute: Determinar OrientaÇÕes das Arestas (H).

6. Baseado no conhecimento das informações relativas à incidência e orientações das arestas, desenhe a representação retangular.

\section{Algoritmo Determinar OrientaÇÕes das Arestas}

ENTRADA: 4-extensão $\mathbb{H}$.

SAÍDA: Define para cada aresta $\alpha$ de $\mathbb{H}$, se $\alpha$ é horizontal ou vertical.

Caso 0: $\quad$ Se $v_{N} v_{S} \in E(\mathbb{H})$ ou $v_{O} v_{L} \in E(\mathbb{H})$ então

/* O grafo original $\mathbb{G}$ contém um circuito que não é face */

Devolva falha;

Caso 1: $\quad$ Caso $\mathbb{H}$ seja o caso trivial contendo 5 vértices e 8 arestas (figura 4.21): As arestas $v_{N} v$ e $v_{S} v$ são horizontais, enquanto as arestas $v_{O} v$ e $v_{L} v$ são verticais;

Caso 2: $\quad$ Algum vértice adjacente a $v_{\infty}$ em $\mathbb{H}$ possui grau 4; Suponha que seja o vértice $v_{L}$ (figura 4.22). A orientação das arestas $v_{N} v$ e $v_{S} v$ são horizontais e a aresta $v v_{L}$ é vertical. Seja $\mathbb{H}^{\prime}=\mathbb{H}-v_{L}$ mais a aresta $v_{L} v_{\infty}$.

Determinar OrientaÇÕes das $\operatorname{ArEstas}\left(\mathbb{H}^{\prime}\right)$

Se não existe uma representação retangular de $\mathbb{H}^{\prime}$ então

Devolva falha;

Caso 3: $\quad$ Todos os vértices adjacentes a $v_{\infty}$ em $\mathbb{H}$ possuem grau 5; (figura 4.10)

Se $v_{1} v_{3} \in E(\mathbb{H})$ então

$C=\left\langle v_{\infty}, v_{L}, v_{1}, v_{3}, v_{O}, v_{\infty}\right\rangle ; / *$ Note que $C$ é um circuito separador */

\section{Senão}

Considere $C=\left\langle v_{\infty}, v_{O}, w_{1}, \ldots, w_{n}, v_{L}, v_{\infty}\right\rangle$ é o menor circuito separador de $\mathbb{G}$ que separa $v_{1}$ de $v_{3}$; (figura 4.13)

Determine a orientação para cada arestas de $v_{O}$ a $v_{L}$ como horizontais;

Crie os grafos $\mathbb{H}^{\prime}$ e $\mathbb{H}^{\prime \prime}$ conforme ilustrado na figura 4.14;

Determinar OrientaÇões das $\operatorname{ArEstas}\left(\mathbb{H}^{\prime}\right)$;

Determinar OrientaÇÕES DAS Arestas( $\left(\mathbb{H}^{\prime \prime}\right)$; 
Se não existe uma representação retangular de $\mathbb{H}^{\prime}$ ou $\mathbb{H}^{\prime \prime}$ então Devolva falha;

A complexidade computacional do algoritmo Koźmiński e Kinnen é limitada pela complexidade do procedimento Determinar Orientações das Arestas. Pois, os passos $1,2,3,4$ e 6 são realizados em tempo linear na quantidade de arestas.

O procedimento Determinar OrientaÇões das Arestas define ao menos uma orientação, logo ele é executado $|E(\mathbb{H})|$ vezes. Como a complexidade para encontrar um caminho é $O(|E(\mathbb{H})|)$, então a complexidade é igual a $O\left(|E(\mathbb{H})|^{2}\right)=O\left(|V(\mathbb{H})|^{2}\right)$.

Logo, a complexidade do algoritmo Koźmiński e Kinnen é $O\left(n^{2}\right)$, onde $n=|V(\mathbb{G})|$.

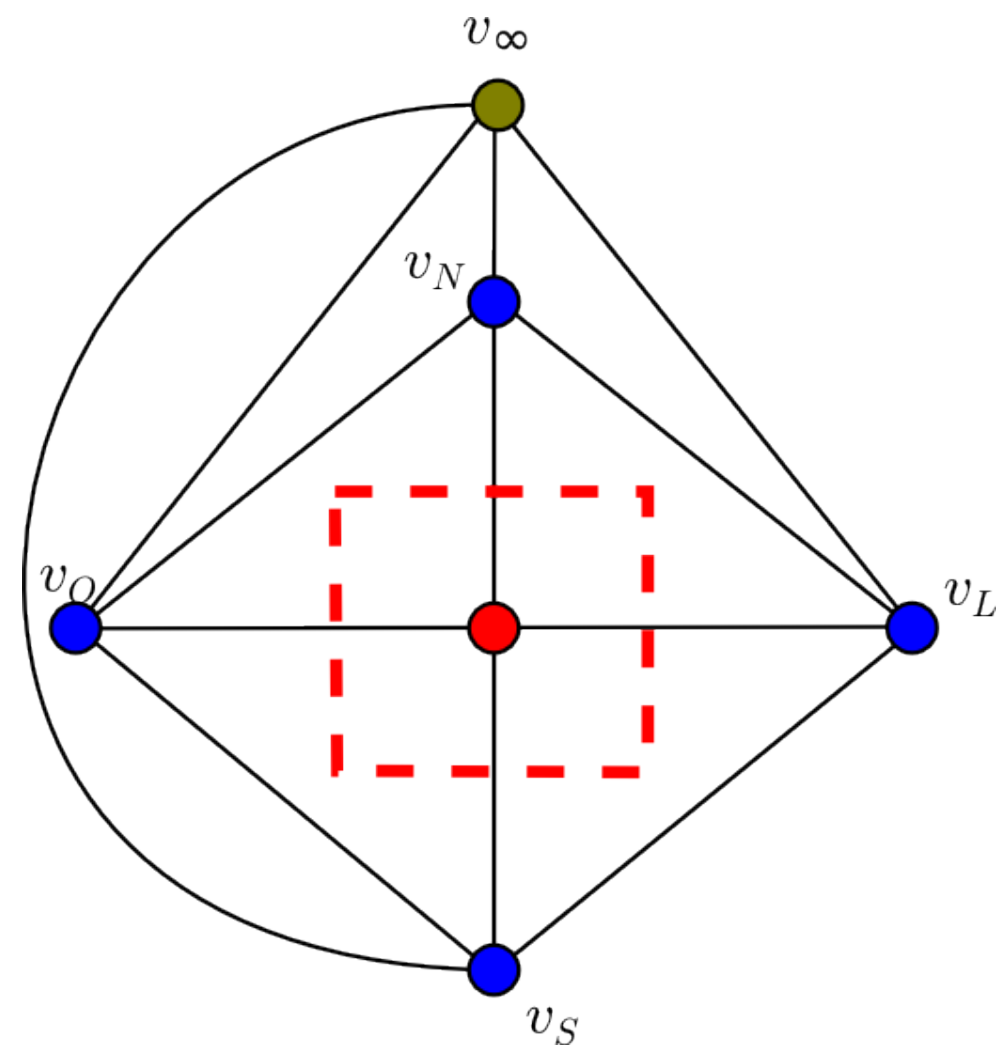

Figura 4.21: 4-extensão trivial. As orientações das arestas são representadas pelas linhas pontilhadas. [KK84] 


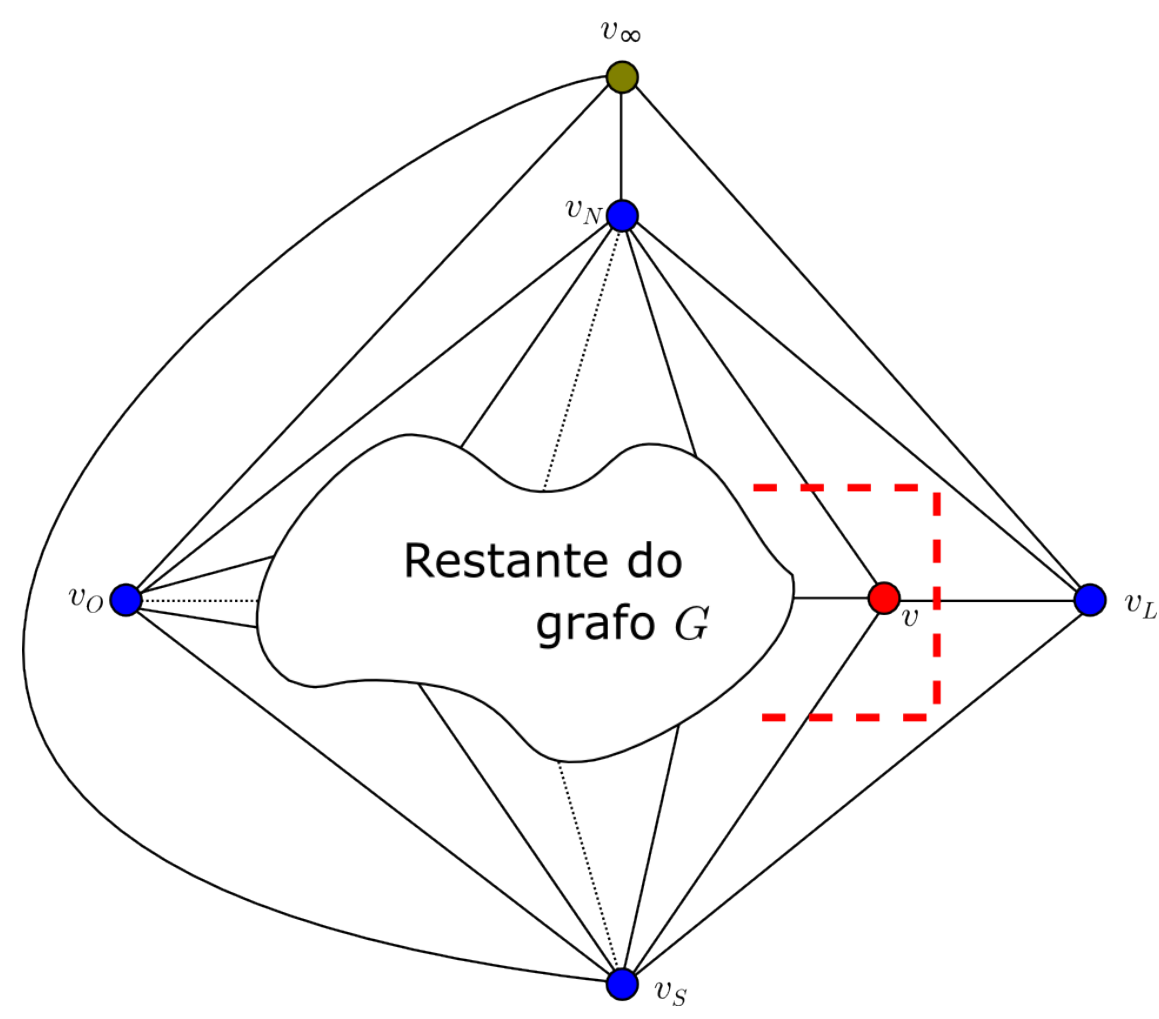

Figura 4.22: 4-extensão que contém um vértice de grau 4 adjacente a $v_{\infty}$. As orientações das arestas são representadas pelas linhas pontilhadas.[KK84] 


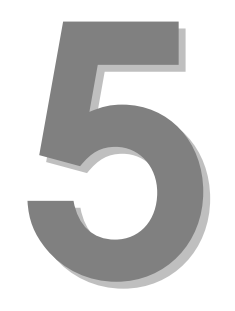

\section{Algoritmo DE BHASKer E SHANi}

Neste capítulo iremos apresentar o algoritmo desenvolvido por Bhasker e Shani [BS88] para solucionar o problema de construção da representação retangular de um grafo plano triangulado. Este algoritmo é baseado na caracterização apresentada no capítulo 4 e no trabalho [BS87] desenvolvido anteriormente por Bhasker e Shani.

O algoritmo que iremos apresentar neste capítulo objetiva solucionar o problema da construção da representação retangular e é dividido em dois procedimentos principais: encontrar um determinado subgrafo do grafo de entrada e desenhar a representação retangular utilizando este subgrafo encontrado.

Considere neste capítulo que o grafo plano triangulado da entrada possui uma representação retangular.

\subsection{GRAFo DOS CAMINHOS DIRIGIDOS}

Seja $\mathbb{G}$ um grafo plano triangulado que possui uma representação retangular $\mathbb{R}$. Pela definição da representação retangular, cada vértice de $\mathbb{G}$ possui um retângulo correspondente em $\mathbb{R}$. Queremos encontrar um subgrafo $\mathbb{G}^{\prime}$ de $\mathbb{G}$. Ainda, desejamos orientar suas arestas de modo que cada aresta represente uma relação de "acima de" entre os retângulos da representação retangular. Se considerarmos que as arestas de $\mathbb{G}^{\prime}$ já estão orientadas, se 
$u v \in E\left(\mathbb{G}^{\prime}\right)$, então o retângulo associado a $u$ está acima do retângulo associado a $v$. Observe que os vértices que representam os retângulos que não possuem nenhum outro retângulo acima, ou seja, os retângulos que incidem no lado superior da representação retangular, em $\mathbb{G}^{\prime}$, não existe nenhuma arestas entrando nestes vértices. Vamos adicionar o vértice $v_{\infty}$ a $\mathbb{G}^{\prime}$ e ligá-lo a cada vértice que representa um retângulo incidente no lado superior da representação retangular. É fácil ver que o grafo resultante é uma família de caminhos que partem do vértice $v_{\infty}$ e terminam nos vértices que representam os retângulos que estão abaixo de todos os outros.

Vamos chamar este grafo que possui o vértice $v_{\infty}$ e todas as arestas são orientadas respeitando a relação de "acima de" de grafo dos caminhos dirigidos da representação retangular $\mathbb{R}$ de $\mathbb{G}$. A figura $5.1 \mathrm{~A}$ ilustra um grafo plano triangulado que possui a representação retangular apresentada na figura 5.1C. O grafo dos caminhos dirigidos da representação retangular $5.1 \mathrm{C}$ do grafo apresentado na figura 5.1A é ilustrado na figura 5.1B.

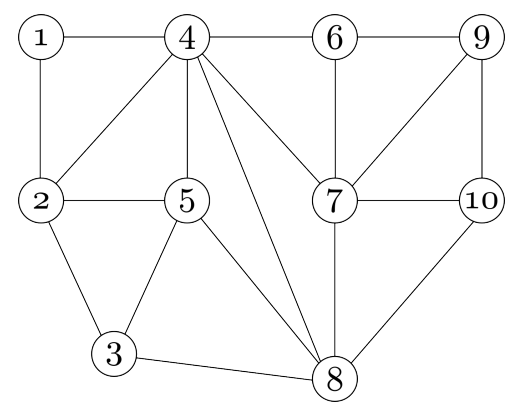

(A)

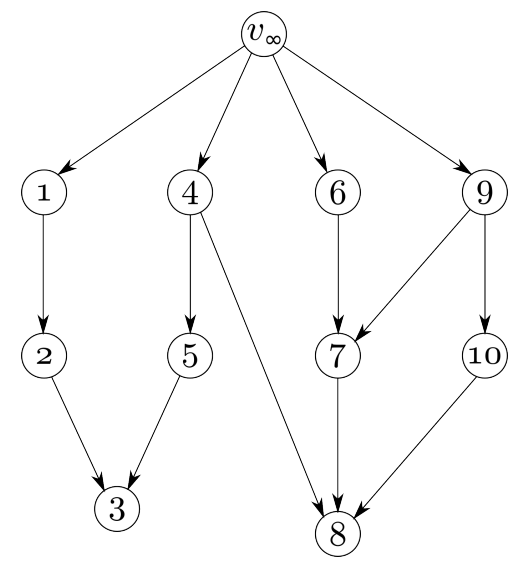

(B)

\begin{tabular}{|l|l|l|l|l|l|}
$A$ & $B$ & $C$ & $D$ & $E$ & $F$ \\
\hline 1 & & & 6 & 9 & \\
\hline 2 & & & 7 & & 10 \\
\cline { 1 - 4 } & 5 & & & & \\
\hline 3 & & & 8 & & \\
\hline
\end{tabular}

(C)

Figura 5.1: (A) Grafo plano triangulado. (B) Grafo dos caminhos dirigidos de (A). (C) Representação retangular de (A) a partir de (B).

Seja $\mathbb{D}$ um grafo dos caminhos dirigidos de $\mathbb{G}$. Como $\mathbb{D}-v_{\infty}$ é um subgrafo do grafo plano $\mathbb{G}$, vamos considerar que os filhos de um vértice $v \in V(\mathbb{D})$ estão ordenados da esquerda para direita. Sejam $u$ e $v$ dois vértices de $\mathbb{D}$, dizemos que $u$ é um ancestral distante de $v$ se e só 
se existe um caminho dirigido de $u$ a $v$ em $\mathbb{D}$ que possui tamanho maior ou igual a 2.

Lema 5.1: Seja $\mathbb{G}$ um grafo plano triangulado que admite uma representação retangular $\mathbb{R}$ e $\mathbb{D}$ um grafo de caminhos dirigidos da representação retangular $\mathbb{R}$ de $\mathbb{G}$. Seja $u, v$ dois vértices de $\mathbb{D}$. Se u é um ancestral distante de $v$, então u não é pai de $v$.

Demonstração: Se $u$ é um ancestral distante de $v$, então existe um caminho dirigido $\left\langle u, w_{1}, w_{2}, \ldots, w_{k}, v\right\rangle$, onde $k \geq 2$. em $\mathbb{D}$. Assim, $u$ está imediatamente acima de $w_{1}$, que está acima de $w_{2}$, e assim sucessivamente. Como a representação retangular $\mathbb{R}$ só possui retângulos então não é possível que $u$ esteja imediatamente acima de $v$. Portanto, $u$ não pode ser pai de $v$.

A figura 5.2 exibe uma comparação entre o grafo $\mathbb{D}-v_{\infty}$ com o grafo plano triangulado $\mathbb{G}$. Nesta representação as arestas pontilhadas identificam as arestas que não pertencem a $\mathbb{D}-v_{\infty}$ enquanto as arestas contínuas pertencem a ambos grafos. Ainda, estamos omitindo as orientações das arestas de $\mathbb{D}-v_{\infty}$.

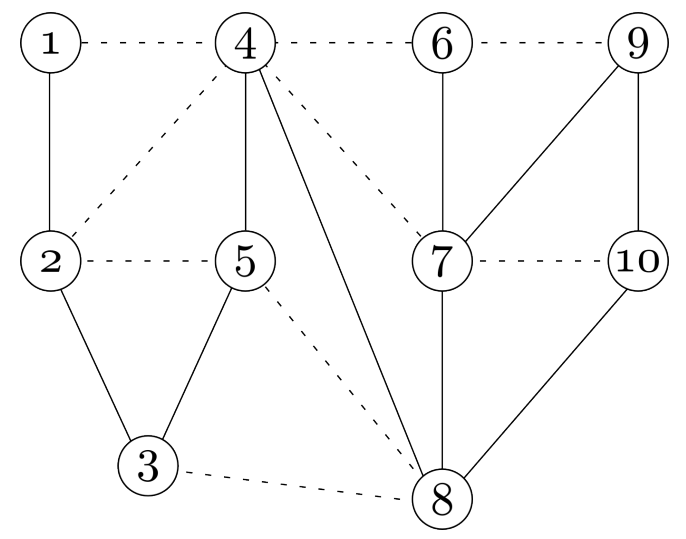

Figura 5.2: Comparação entre o grafo $\mathbb{D}-v_{\infty}$ com o grafo plano triangulado $\mathbb{G}$.

O lema a seguir irá relacionar o grafo plano triangulado com o grafo dos caminhos dirigidos.

Lema 5.2: Seja $\mathbb{G}$ um grafo plano triangulado e $\mathbb{D}$ um grafo dos caminhos dirigidos de uma representação retangular de $\mathbb{G}$. Se uv $\in E(\mathbb{G})$, então:

(1) u não é um ancestral distante de $v$ em $\mathbb{D}$.

(2) v não é um ancestral distante de $u$ em $\mathbb{D}$.

Este lema mostra que ou um dos vértices é pai e outro filho, ou não existe um caminho dirigido entre eles em $\mathbb{D}$. A prova deste resultado é análoga a do lema 5.1. 


\subsection{VisÃo GERAL do Algoritmo DE BHASKER E SHANi}

Considerando que estamos trabalhando com um grafo plano triangulado $\mathbb{G}$ que possui uma representação retangular, iremos apresentar como obter um grafo dos caminhos dirigidos de $\mathbb{G}$. Observe que podem existir diversas representações retangulares de $\mathbb{G}$, logo, pode existir o mesmo número de grafos dos caminhos dirigidos. Nosso objetivo é encontrar um destes grafos e a partir deste construir uma representação retangular de $\mathbb{G}$.

Nesta seção vamos apresentar a estratégia do algoritmo de Bhasker e Shani. Para facilitar o entendimento, inicialmente, vamos adotar o sentido inverso do algoritmo, vamos partir da representação retangular para explicar como o grafo dos caminhos dirigidos é utilizado durante o algoritmo.

Observe a representação retangular ilustrada na figura 5.1C. A representação retangular é particionada em seis colunas (A, B, C, D, E e F) que são limitadas pelas linhas pontilhadas. Note que toda aresta vertical está sobre uma linha pontilhada.

Como já explicado anteriormente, podemos construir um grafo dos caminhos dirigidos $\mathbb{D}$ a partir desta representação retangular (figura 5.1B).

Se olharmos para cada coluna da representação retangular, conseguimos identificar um caminho em $\mathbb{D}$ do vértice $v_{\infty}$ a uma folha de $\mathbb{D}$. Ainda, dada duas colunas, os caminhos referente a elas em $\mathbb{D}$, são disjuntos nas arestas.

Como existe esta correspondência entre os caminhos de $\mathbb{D}$ com as colunas da representação retangular, o algoritmo consiste em receber o grafo plano triangulado $\mathbb{G}$ (figura $5.1 \mathrm{~A}$ ), encontrar um grafo dos caminhos dirigidos dirigidos $\mathbb{D}$ de $\mathbb{G}$ e, por fim, processar $\mathbb{D}$ para encontrar uma representação retangular de $\mathbb{G}$.

Agora, vamos omitir como faremos para encontrar um grafo dos caminhos dirigidos de $\mathbb{G}$. Suponha que sabemos encontrar um grafo dos caminhos dirigidos e este satisfaz o lema 5.2 .

De modo geral, o algoritmo para encontrar uma representação retangular a partir do grafo dos caminhos dirigidos $\mathbb{D}$ percorre todos os caminhos dirigidos do vértice $v_{\infty}$ até uma folha, da esquerda para direita.

Vamos simular o algoritmo para construir uma representação retangular a partir do grafo plano triangulado. Considere como entrada o grafo $\mathbb{G}$ ilustrado na figura 5.1A.

Para construir o grafo dos caminhos dirigidos, precisamos identificar os vértice que irão corresponder aos retângulos dos cantos: superior esquerdo, superior direito, inferior esquerdo e inferior direito. Estes vértices serão rotulados, respectivamente, como $N O, N E, S O$ e $S E$. Para o grafo da figura $5.1 \mathrm{~A}$, temos $N O=1, N E=9, S O=3$ e $S E=8$. 
O circuito $C=\langle 1,4,6,9,10,8,3,2,1\rangle$ define a face externa de $\mathbb{G}$. Podemos particionar o circuito $C$ em quatro caminhos da borda: caminho superior, caminho inferior, caminho esquerdo e caminho direito. Por exemplo, neste grafo o caminho superior da borda possui os vértices 1, 4, 6 e 9; o caminho inferior da borda possui os vértices 3 e 4; o caminho esquerdo da borda possui os vértices 1, 2 e 3; e o caminho direito da borda possui os vértices 9, 10 e 8 .

Após definir os rótulos $N O, N E, S O$ e $S E$ podemos construir um grafo dos caminhos direcionados $\mathbb{D}$ de $\mathbb{G}$. Todos os caminhos deverão começar em um vértice do caminho superior da borda de $\mathbb{G}$ e terminar em um caminho inferior da borda de $\mathbb{G}$.

Inicialmente, o grafo $\mathbb{D}$ só deve possuir o vértice $v_{\infty}$. Devemos percorrer o caminho esquerdo da borda $(\langle 1,2,3\rangle)$ do grafo $\mathbb{D}$. Este caminho irá se tornar o caminho mais a esquerda do grafo $\mathbb{D}$ (figura $5.3 \mathrm{~A}$ ). Como já adicionamos os vértice 1,2 e 3 em $\mathbb{D}$, então podemos remover o caminho esquerdo da borda de $\mathbb{G}$, o grafo $\mathbb{G}$ resultante e os novos rótulos são ilustrados na figura 5.3B.

O novo caminho esquerdo da borda de $\mathbb{G}$ não pode ser adicionado a $\mathbb{D}$ da mesma forma que foi feito anteriormente, pois a aresta $(4,8)$ viola o lema 5.2. O que acontece é que se o caminho $\langle 4,5,8\rangle$ for adicionado a $\mathbb{D}$, então o vértice 4 será um ancestral distante de 8 . Podemos contornar este problema não utilizando a aresta $(5,8)$. Para completar o caminho no grafo $\mathbb{D}$ em um vértice que pertence ao caminho inferior da borda de $\mathbb{G}$, ao invés de adicionarmos a aresta $(5,8)$, vamos substituí-la pela aresta $(5,3)$. A aresta $(4,8)$ será utilizada para construir um outro caminho. O processamento do caminho $\langle 4,5,8\rangle$ irá resultar no grafo dos caminhos dirigidos ilustrado na figura 5.3C. O grafo $\mathbb{G}$ resultante após o processamento é ilustrado na figura 5.3D. Observe que o grafo da figura 5.3B e o grafo da figura 5.3D possuem o mesmo vértice rotulado como $S O$. Isso se deve ao fato que $S O=S E$ e que todos os caminhos devem terminar em um vértice pertencente ao caminho inferior da borda de $\mathbb{G}$.

Neste momento, $\langle 6,7,8\rangle$ é o caminho esquerdo da borda de $\mathbb{G}$. Adicionando este caminho a $\mathbb{D}$, temos que o grafo resultante é igual ao ilustrado na figura $5.4 \mathrm{~A}$. O grafo $\mathbb{G}$ resultante é ilustrado na figura 5.4B. Adicionando este último caminho a $\mathbb{D}$ temos o grafo dos caminhos dirigidos de $\mathbb{G}$ (figura 5.4C). É fácil verificar que $\mathbb{D}$ não viola o lema 5.2. Perceba que o grafo dos caminhos dirigidos da figura 5.4C não é igual ao ilustrado na figura 5.1B, isto se deve ao fato que um grafo plano triangulado pode possuir diversas representações retangulares.

É importante ressaltar que o algoritmo para obter um grafo dos caminhos dirigidos é mais complicado do que apresentado anteriormente, pois, precisamos tratar diversos casos especiais, como mostraremos a seguir.

Depois de construirmos $\mathbb{D}$ a partir de $\mathbb{G}$, podemos construir a representação retangular de $\mathbb{G}$. 


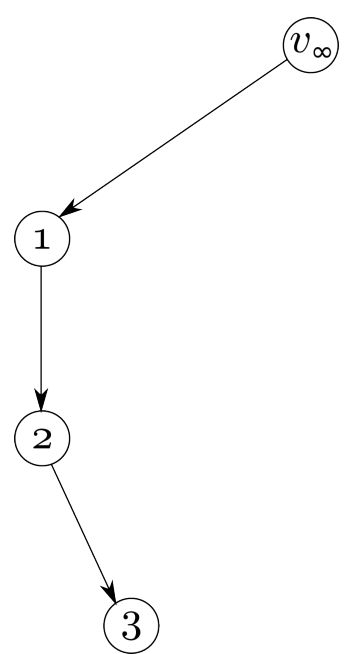

(A)

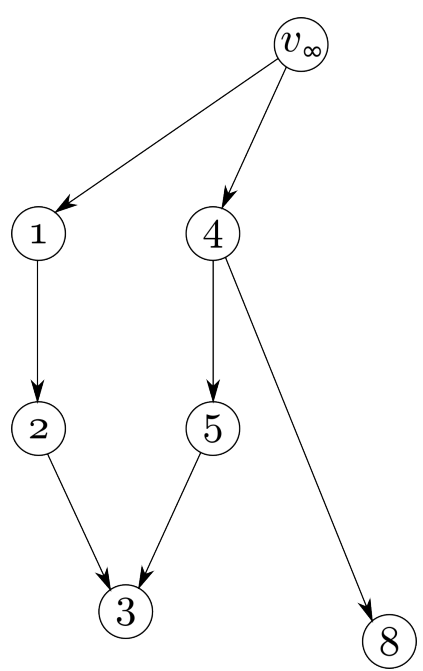

(C)

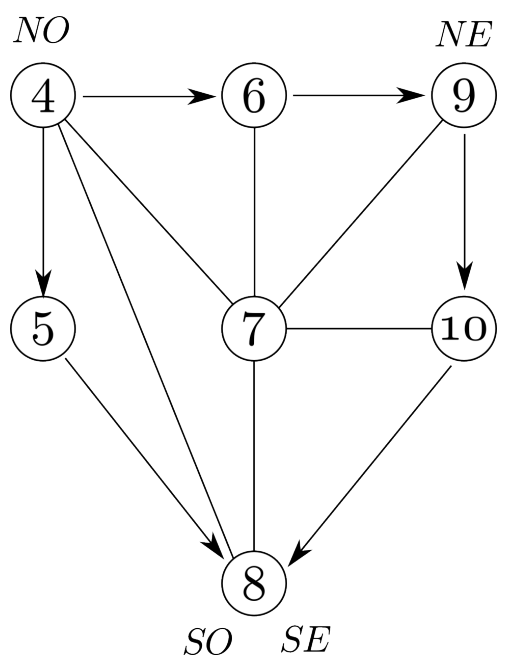

(B)

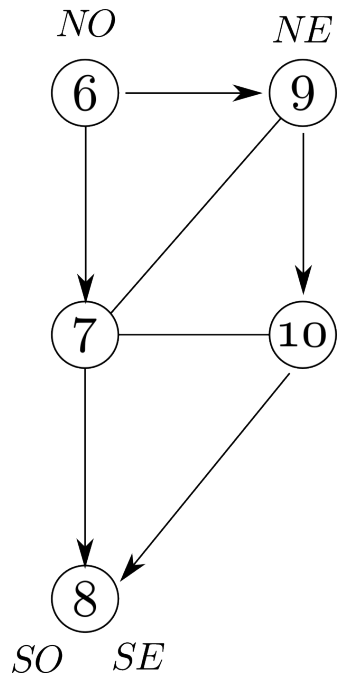

(D)

Figura 5.3: Exemplo de uma construção do grafo dos caminhos dirigidos [BS88].

Vamos percorrer os caminhos do vértice $v_{\infty}$ até uma folha de $\mathbb{D}$ da esquerda para direita. Para simplificar a notação, sempre que nos referirmos ao retângulo $X$, estamos nos referindo ao retângulo associado ao vértice $X$.

Inicialmente, o caminho mais esquerdo de $\mathbb{D}$ irá gerar os retângulos da coluna A (figura 5.5A). Vamos definir a largura de todos os retângulos desta coluna como sendo 1 unidade, entretanto, durante a execução do algoritmo podemos modificar a largura de algum retângulo que pertence a esta coluna. O próximo caminho é $\left\langle v_{\infty}, 4,5,3\right\rangle$. Como o retângulo 4 é adjacente aos retângulos que já foram definidos (retângulos 1 e 2), devemos desenhar o retângulo 4 conforme ilustrado na figura 5.5B. Desenhar o retângulo 4 desta forma possibilita satisfazer as restrições de adjacência do vértice 5. O retângulo 5, deve ser adjacente, além do retângulo 2, ao retângulo 3. Entretanto, o retângulo 3 já foi desenhado e como o retângulo 5 deve estar acima dele, precisamos alargá-lo de modo que satisfaça esta condição (figura 5.5C), finalizando a coluna B. O próximo caminho é $\left\langle v_{\infty}, 4,8\right\rangle$. Como o retângulo 4 já foi desenhado, 


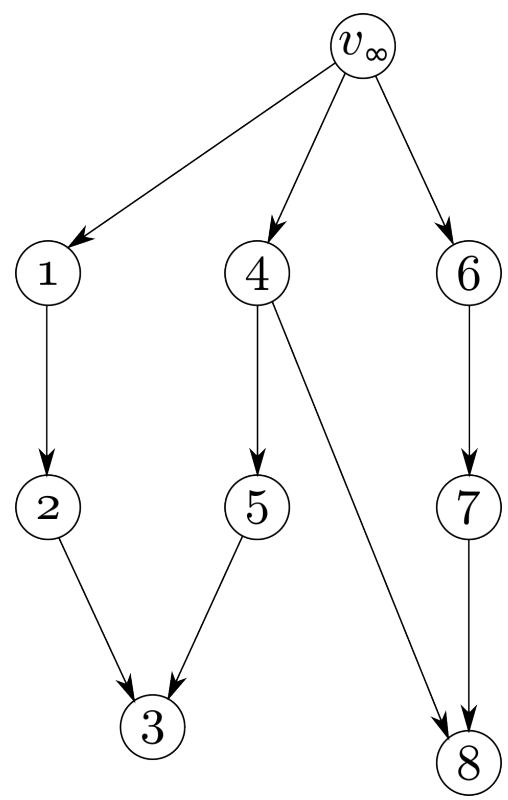

(A)
NO NE

(9)

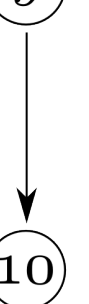

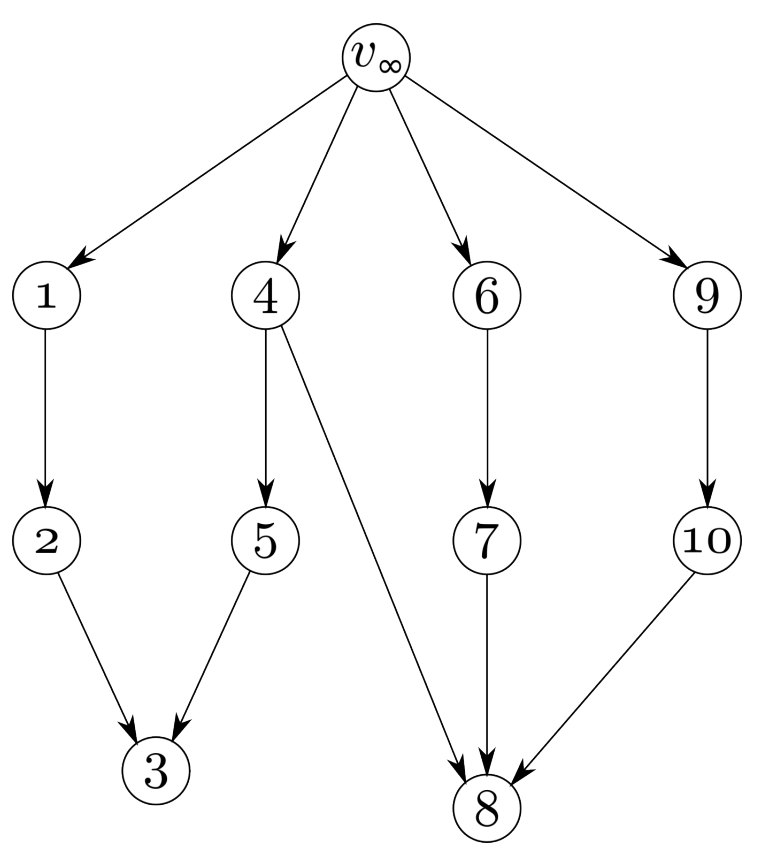

(C)

Figura 5.4: Exemplo de uma construção do grafo dos caminhos dirigidos (continuação)[BS88].

precisamos alargá-lo para poder colocá-lo acima do retângulo 8. Assim, concluímos a coluna C (figura 5.5D).

Para coluna $\mathrm{D}$, iremos percorrer o caminho $\left\langle v_{\infty}, 6,7,8\right\rangle$. O retângulo 6 deve ficar no topo, seguido pelo retângulo 7. Como o retângulo 7 deve ficar acima do retângulo 8, vamos alargar o retângulo 8 (figura $5.6 \mathrm{~A}$ ). O último caminho de $\mathbb{D}$ é $\left\langle v_{\infty}, 9,10,8\right\rangle$ será responsável pela última coluna da representação retangular (E). Como nos caminhos anteriores, vamos desenhar os retângulos 9 e 10. Já o retângulo 8, precisamos alargá-lo para que fique abaixo de 10 (figura 5.6B). Como este era o último caminho de $\mathbb{D}$, então após processá-lo, temos 


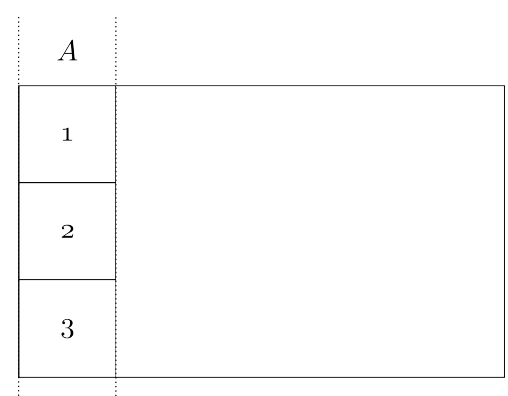

(A)

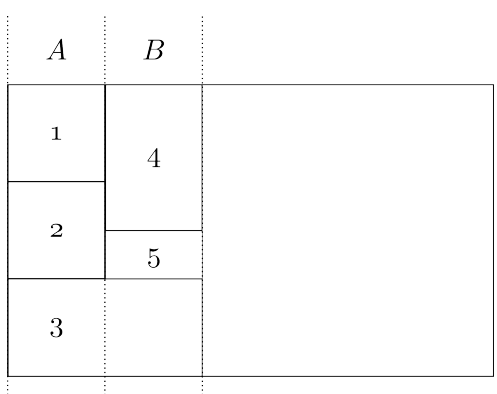

(C)

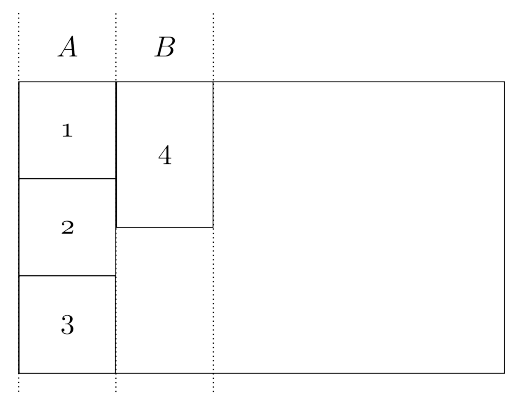

(B)

\begin{tabular}{|l|l|l|l|}
$A A$ & $B$ & $C$ \\
\hline 1 & & & \\
\cline { 1 - 1 } 2 & 4 & \\
\cline { 1 - 2 } & 5 & \multirow{2}{*}{8} \\
\cline { 1 - 2 } 3 & & \\
\hline
\end{tabular}

(D)

Figura 5.5: Exemplo de uma construção da representação retangular de $\mathbb{G}$.[BS88].

uma representação retangular de $\mathbb{G}$.

\begin{tabular}{|c|c|c|c|}
\hline$A$ & $B$ & $C$ & $D$ \\
\hline 1 & \multirow{2}{*}{4} & & 6 \\
\hline \multirow{2}{*}{2} & & & 7 \\
\hline & 5 & & \\
\hline 3 & & & 8 \\
\hline
\end{tabular}

(A)

\begin{tabular}{|c|c|c|c|c|}
\hline$A$ & $B$ & $C$ & $D$ & $E$ \\
\hline 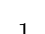 & \multirow{2}{*}{4} & & 6 & 9 \\
\hline \multirow{2}{*}{2} & & & 7 & 10 \\
\hline & 5 & & & \\
\hline 3 & & & 8 & \\
\hline
\end{tabular}

(B)

Figura 5.6: Exemplo de uma construção da representação retangular de $\mathbb{G}$. (continuação) [BS88].

\subsection{Algoritmo PARA CONSTRUir UM GRAFO DOS CAMINHOS DIRIGIDOS}

Seja $\mathbb{G}$ um grafo plano triangulado que possui uma representação retangular. Então, existe um grafo dos caminhos dirigidos $\mathbb{D}$ de $\mathbb{G}$.

Vamos construir um grafo dirigido $\mathbb{D}$ a partir de $\mathbb{G}$ que representa a relação "acima de". Ou seja, $\mathbb{D}$ vai ser uma orientação de um subgrafo de $\mathbb{G}$ de tal forma que o arco $(u, v)$ pertence a $\mathbb{D}$ se e só se o retângulo correspondente a $u$ está em cima do retângulo correspondente a 
v. A construção deste grafo será descrita a seguir.

Como todos os vértices de $\mathbb{G}$ devem pertencer a $\mathbb{D}$, iremos processar um vértice $v \in V(\mathbb{G})$. O processamento consistem em descobrir como devemos adicioná-lo a $\mathbb{D}$ de modo que todas as arestas que incidem em $v$ não violem o lema 5.2.

O algoritmo consiste em receber um subgrafo $\mathbb{G}^{\prime}$ do grafo plano triangulado $\mathbb{G}$, com 4 vértices da face externa de $\mathbb{G}^{\prime}$ rotulados $\left(N O_{\mathbb{G}^{\prime}}, N E_{\mathbb{G}^{\prime}}, S O_{\mathbb{G}^{\prime}}\right.$ e $\left.S E_{\mathbb{G}^{\prime}}\right)$ que particionam o circuito da face em 4 caminhos disjuntos nas arestas. O objetivo do algoritmo é encontrar uma cobertura de caminhos dirigidos de $\mathbb{G}^{\prime}$ para adicionarmos em $\mathbb{D}$. Vamos considerar que iremos adicionar estes caminhos como filho do vértice VerticeInicial $\mathbb{G}_{\mathbb{G}^{\prime}}$ e devem terminar no vértice VerticeFinal G $_{\mathbb{G}^{\prime}}$, onde VerticeFinal $_{\mathbb{G}^{\prime}}$, VerticeInicial $_{\mathbb{G}^{\prime}} \in V(\mathbb{D})$. Basicamente, iremos adicionar em $\mathbb{D}$, uma aresta entre o Vertice Inicial $\mathbb{G}_{\mathbb{G}^{\prime}}$ e o primeiro vértice de cada caminho e uma aresta entre o último vértice de cada caminho e o vértice VerticeFinal $_{\mathbb{G}^{\prime}}$. Note que em alguns momentos não iremos definir o vértice VerticeFinal $_{\mathbb{G}^{\prime}}$ e consequentemente, não iremos adicionar as arestas que saem do último vértice de cada caminho. Para denotar esta situação, iremos definir VerticeFinal $\mathbb{G}_{\mathbb{G}^{\prime}}=N U L O$.

Inicialmente, $\mathbb{G}^{\prime}=\mathbb{G}$ e os rótulos $N O_{\mathbb{G}^{\prime}}=N O, N E_{\mathbb{G}^{\prime}}=N E, S O_{\mathbb{G}^{\prime}}=S O$ e $S E_{\mathbb{G}^{\prime}}=S E$. Ainda, VerticeInicial $\mathbb{G}_{\mathbb{G}^{\prime}}=v_{\infty}$ e VerticeFinal $_{\mathbb{G}^{\prime}}=N U L O$.

O algoritmo consiste em analisar o caminho esquerdo da borda de $\mathbb{G}^{\prime}$. Este caminho é orientado e sua orientação é definida pelos rótulos $N O_{\mathbb{G}^{\prime}}$ e $S O_{\mathbb{G}^{\prime}}$ (figura 5.7). A cada iteração, iremos processar um vértice $v$ deste caminho, seguindo a ordem definida pelo caminho. Processar um vértice deste caminho consiste em adicionar este ao grafo $\mathbb{D}$, caso ainda não tenha sido adicionado e verificar se todas as arestas que incidem nele não violam o lema 5.2. Após processar $v$, iremos remover ao menos uma aresta de $\mathbb{G}^{\prime}$, e caso $v$ seja o primeiro ou o último vértice do caminho da borda esquerda $\left(v=N O_{\mathbb{G}^{\prime}}\right.$ ou $\left.v=S O_{\mathbb{G}^{\prime}}\right)$ podemos optar por não removê-lo de $\mathbb{G}^{\prime}$. É importante ressaltar que sempre que removemos uma aresta ou um vértice de $\mathbb{G}^{\prime}$, iremos alterar a borda esquerda de $\mathbb{G}^{\prime}$ para que o grafo resultante possua uma borda esquerda distinta da atual.

Observe que no grafo $\mathbb{G}^{\prime}$ os vértices do caminho esquerdo da borda podem possuir arestas que incidem em vértices que não pertencem ao caminho esquerdo. Caso não exista aresta distinta das arestas do caminho esquerdo que incide, ou em um vértice do caminho, ou em um vértice diferente do caminho ( $\mathbb{G}^{\prime}$ é somente um caminho), podemos adicionar este caminho a $\mathbb{D}$ e não existirão dois vértice que violam o lema 5.2. A figura 5.8 ilustra uma aresta que incide em vértices pertencentes ao caminho esquerdo da borda que irá violar o lema 5.2 caso adicionarmos o caminho esquerdo a $\mathbb{D}$. A aresta pontilhada é a aresta problemática. 


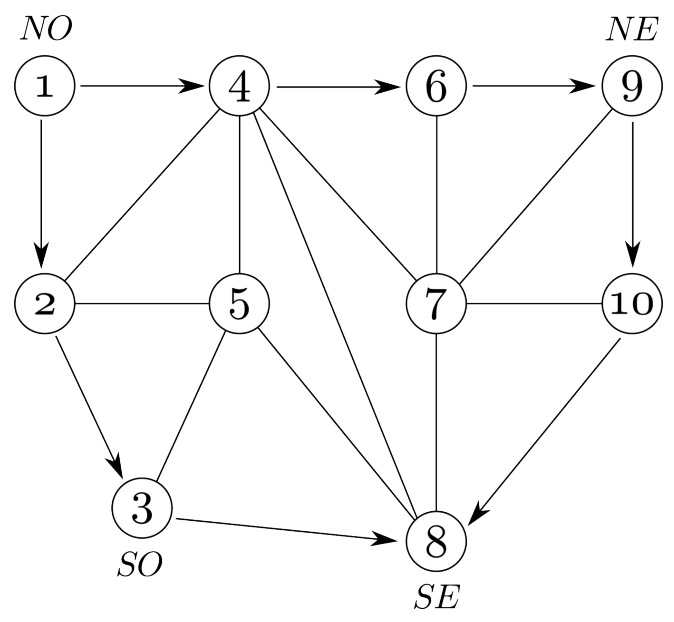

Figura 5.7: Orientação das arestas da borda do grafo plano triangulado.

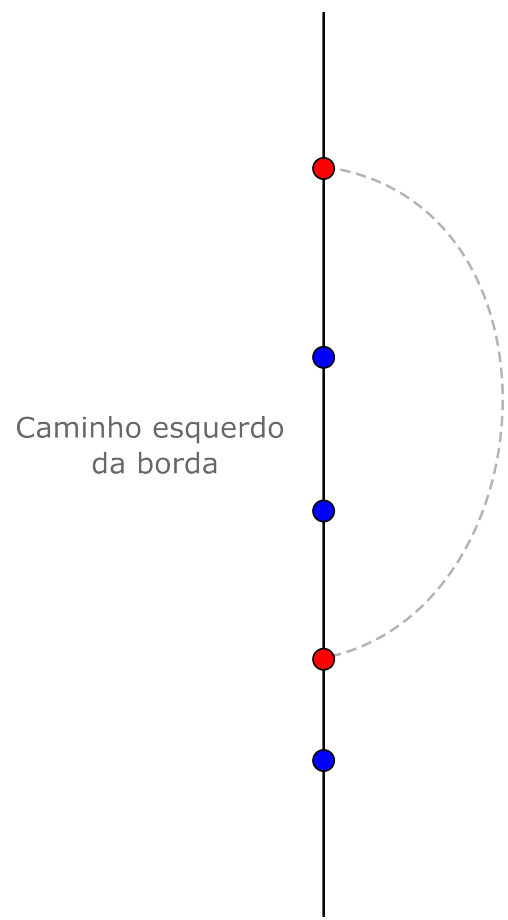

Figura 5.8: Exemplo de aresta que incide em vértices pertencentes ao caminho esquerdo da borda que irá violar o lema 5.2 caso adicionarmos o caminho esquerdo a $\mathbb{D}$.

Logo, não podemos simplesmente adicionar o caminho esquerdo da borda como filho de VerticeInicial $_{\mathbb{G}^{\prime}}$ e terminando em VerticeFinal $\mathbb{G}_{\mathbb{G}^{\prime}}$ em $\mathbb{D}$.

Para encontrar uma cobertura de caminhos do grafo $\mathbb{G}^{\prime}$ que satisfaça o lema 5.2, devemos processar o caminho esquerdo da borda avaliando, a partir da ordem induzida pelo caminho esquerdo da borda atual, vértice por vértice. Iremos repetir este processo (modificando o grafo $\mathbb{G}^{\prime}$ ) até que todos os vértice de $\mathbb{G}^{\prime}$ sejam adicionados a $\mathbb{D}$. Assim, em qualquer iteração, precisamos saber quem é o vértice que está sendo processado e qual foi o último vértice que foi processado. Caso estejamos processando o primeiro vértice do caminho (vértice rotulado por NO), não irá existir um vértice anterior a este. 
A partir de agora, vamos denotar por $v$ o vértice do caminho esquerdo da borda que estamos processando e $\operatorname{pred}_{v}$ o último vértice processado (caso $\operatorname{pred}_{v}$ não exista, então pred $_{v}$ será igual a $N U L O)$.

Vamos dividir a análise de como processar $v$ em dois casos: se $v$ pertence à borda direita de $\mathbb{G}^{\prime}$ e se $v$ não pertence à borda direita.

Suponha que o vértice $v$ não pertença à borda direita.

Como $v$ não está na borda direita de $\mathbb{G}^{\prime}$, então existe ao menos um vértice adjacente a $v$ que não pertence à borda esquerda de $\mathbb{G}^{\prime}$. Seja $q$ o primeiro vértice adjacente a $v$ após pred no sentido horário em $\mathbb{G}^{\prime}$.

Vamos percorrer os vértices adjacente a $v$ em $\mathbb{G}^{\prime}$ no sentido horário, iniciando pelo primeiro vértice após $q$. Seja $u$ este vértice.

Se $u$ é o próximo vértice do caminho esquerdo da borda, podemos adicionar $v$ a $\mathbb{D}$ e remover $v$ de $\mathbb{G}^{\prime}$.

Se $u$ pertence à borda superior de $\mathbb{G}^{\prime}$, então temos a configuração da figura 5.9. Considere o subgrafo de $\mathbb{G}^{\prime}$ referente à "região esquerda superior" destacada na figura como o grafo $\mathbb{H}$.

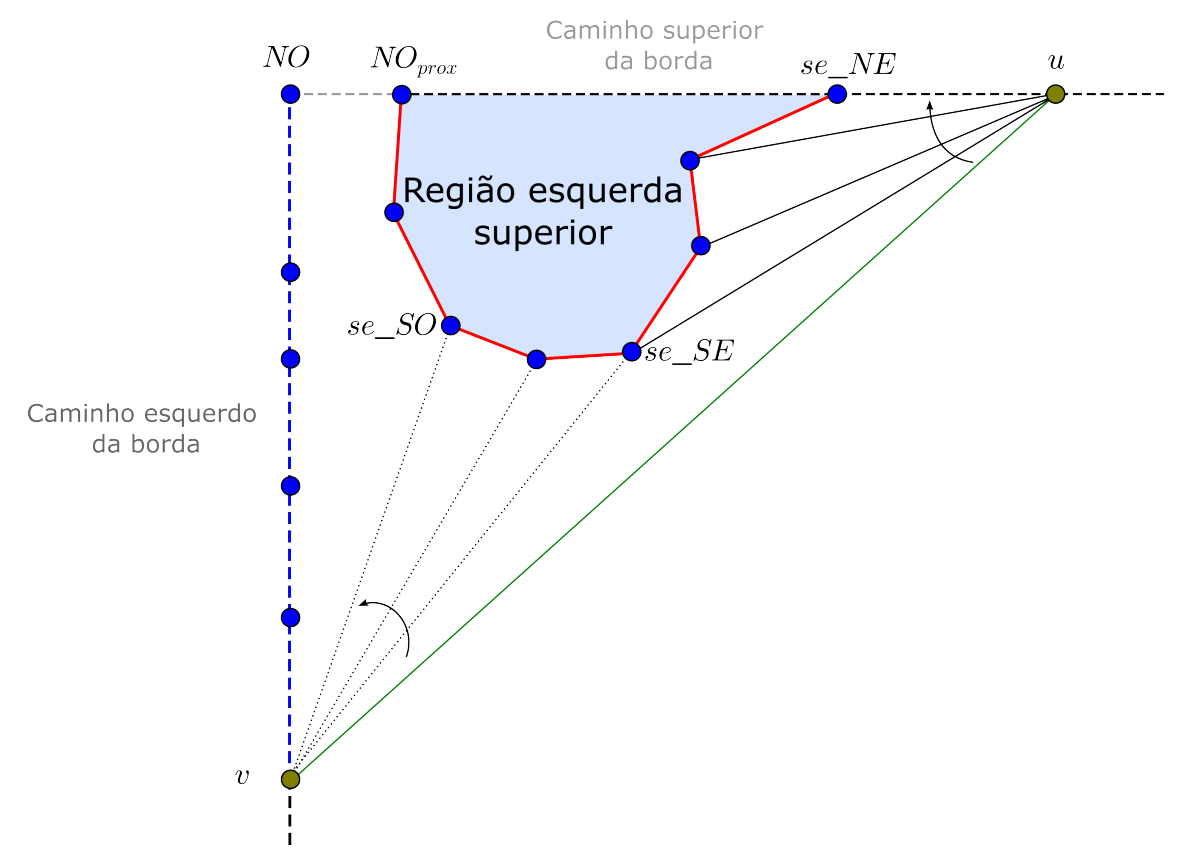

Figura 5.9: Ilustração da região esquerda superior. [BS88]

Precisamos processar o subgrafo $\mathbb{H}$ e garantir que $u$ não seja um ancestral distante de $v$, pois senão a aresta $u v$ violaria o lema 5.2. Vamos rotular os vértices ilustrados na figura 5.9 da seguinte forma: $N O_{\mathbb{H}}=N O_{\text {prox }}, S O_{\mathbb{H}}=s e_{-} S O, N E_{\mathbb{H}}=s e_{-} N E$ e $S E_{\mathbb{H}}=s e_{-} S E$. Perceba que $s e \_S O$ é o primeiro vértice adjacente a $v$ no sentido horário após pred $_{v}$. 
Todos os caminhos que cobrem $\mathbb{H}$ devem ser adicionados a $\mathbb{D}$ de modo que sejam filhos de VerticeInicial $_{\mathbb{G}^{\prime}}$ e devem terminar no vértice $v$. Perceba que o retângulo correspondente a $v$ deve ficar abaixo de todos os retângulos correspondentes aos vértices de $\mathbb{H}$.

Depois, removemos o grafo $\mathbb{H}$ de $\mathbb{G}^{\prime}$ e continuamos a processar os vértices adjacentes à $v$.

A figura 5.10 ilustra como o grafo $\mathbb{G}^{\prime}$ deverá ficar após o processamento de $\mathbb{H}$.

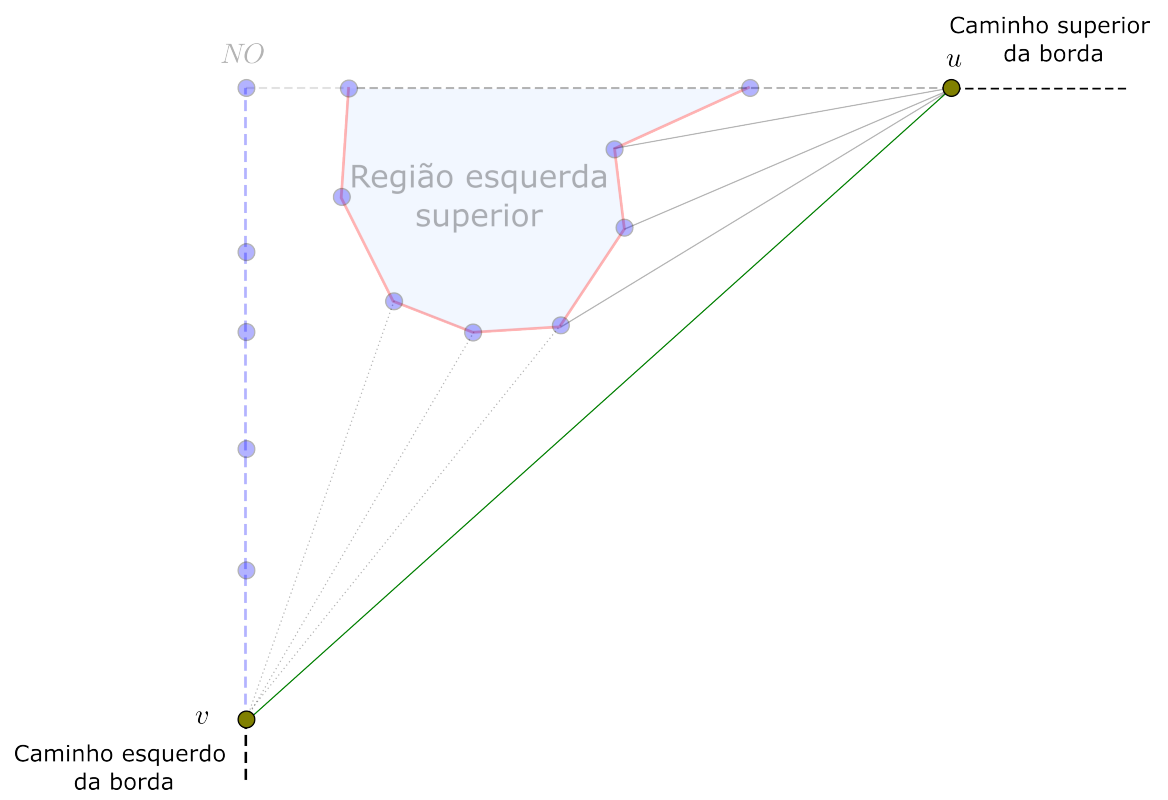

Figura 5.10: Grafo resultante após o processamento da região esquerda superior.

Um caso semelhante ao que tratamos é quando o vértice $u$ é diferente de $S O_{\mathbb{G}^{\prime}}$, não pertence a $\mathbb{D}$ e pertence à borda inferior de $\mathbb{G}^{\prime}$. A figura 5.11 ilustra este caso.

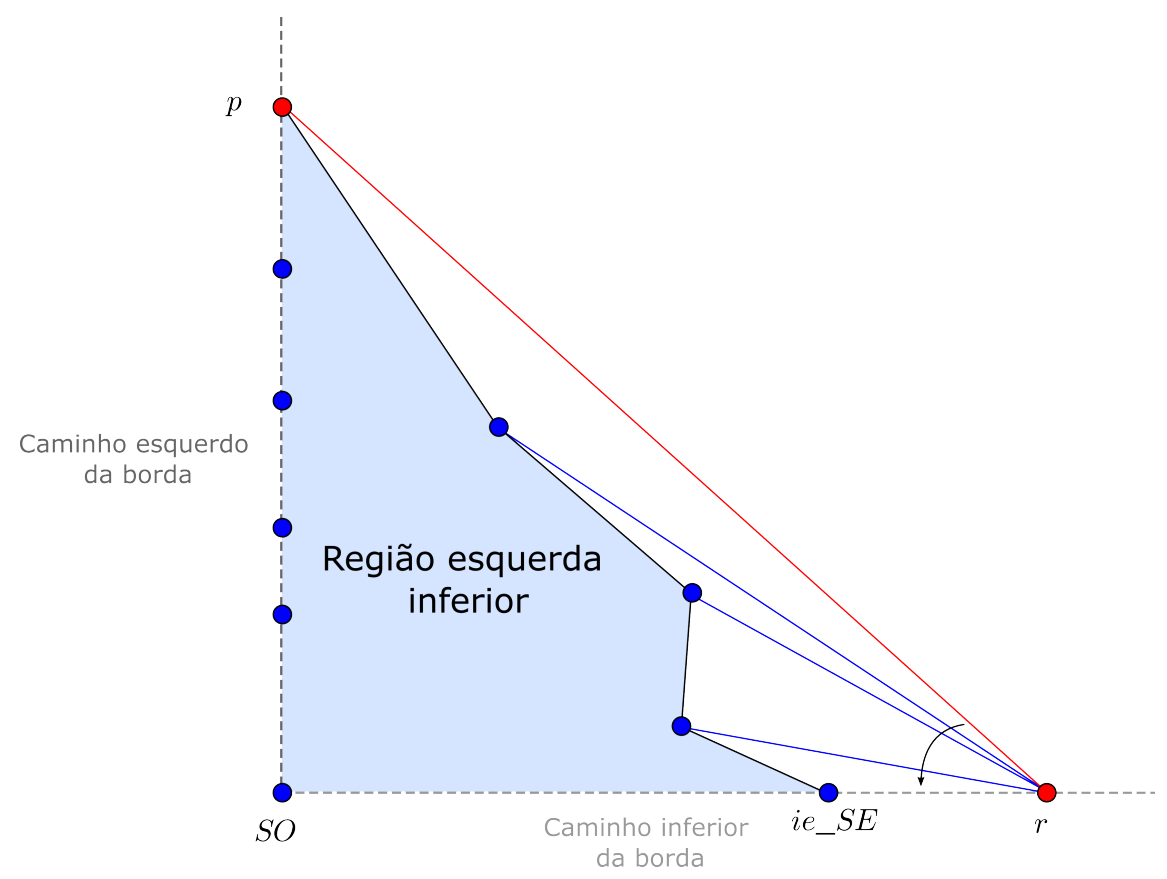

Figura 5.11: Ilustração da região esquerda inferior. [BS88] 
O subgrafo $\mathbb{H}$ equivalente à região identificada como "região inferior esquerda" na figura 5.11 deve ser processado de modo que garantimos que $v$ não seja ancestral distante de $u$.

É fácil ver que em $\mathbb{D}, v$ deve ser ancestral, não necessariamente distante, de todos os vértices de $\mathbb{H}$, pois o retângulo correspondente a $v$ está acima dos correspondentes aos vértices de $\mathbb{H}$. Ainda, para evitar que a aresta $v u$ viole o lema 5.2, precisamos garantir que $u$ seja filho de $v$ e não exista nenhum caminho em $\mathbb{D}$ entre um vértice de $\mathbb{H}$ até o vértice $u$. Considere os seguintes rótulos para o subgrafo $\mathbb{H}: N O_{\mathbb{H}}=N E_{\mathbb{H}}=p, S O_{\mathbb{H}}=S O_{\mathbb{G}^{\prime}}$ e $S E_{\mathbb{H}}=i e_{-} S E$.

Todos os caminhos que cobrem $\mathbb{H}$ deverão ser adicionados a $\mathbb{D}$ de modo que sejam filhos de $v$ e vem terminar no VerticeFinal $\mathbb{G}_{\mathbb{G}^{\prime}}$. A figura 5.12 ilustra o grafo $\mathbb{G}^{\prime}$ resultante.

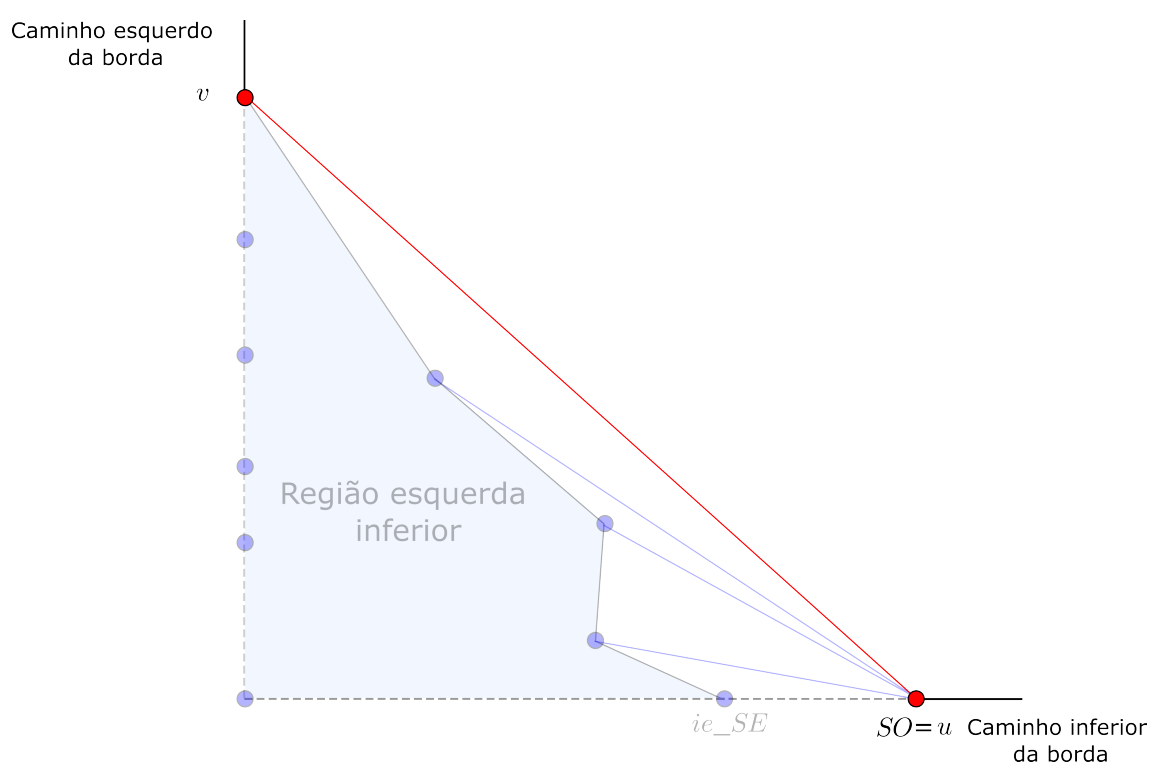

Figura 5.12: Grafo resultante após o processamento da região esquerda inferior.

Para finalizar este caso, adicionamos $u$ como filho de $v$ em $\mathbb{D}$, ainda, caso o vértice VerticeFinal $_{\mathbb{G}^{\prime}}$ for diferente de $N U L O$, adicionamos a aresta $\left(u\right.$, VerticeFinal $\left._{\mathbb{G}^{\prime}}\right)$ a $\mathbb{D}$. 
Agora, vamos tratar o caso de $u$ pertencer à borda esquerda de $\mathbb{G}^{\prime}$, de modo que $u$ não seja o próximo vértice do caminho esquerdo da borda de $\mathbb{G}^{\prime}$. Considere a ordenação do caminho esquerdo da borda de $\mathbb{G}$. Existem dois casos a serem tratados: $(i)$ existe um caminho com mais que uma aresta de $v$ a $u$; e (ii) existe um caminho com mais que uma aresta de $u$ a $v$.

Primeiramente, vamos tratar o caso $i$, onde o retângulo $v$ está "acima" do retângulo $u$.

Se $u$ pertence a $\mathbb{D}$, então $u$ é o vértice $S O_{\mathbb{G}^{\prime}}$, pois este é o único vértice do caminho esquerdo que está abaixo de $v$ que pode ter sido adicionado a $\mathbb{D}$ anteriormente. Neste caso, precisamos tratar uma componente pendente $\mathbb{H}$. A seguir, vamos apresentar uma forma genérica de como tratar uma componente pendente.

Uma componente pendente ocorre quando estamos processando um vértice $c p \_r$ e existe um vértice $c p \_p$ adjacente a $c p \_r$ em $\mathbb{G}^{\prime}$ de modo que $c p \_p$ já foi adicionado a $\mathbb{D}$.

Logo, se $c p \_p$ pertence a $\mathbb{G}^{\prime}$, então $c p \_p$ pertenceu a alguma borda esquerda de algum subgrafo $\mathcal{F}$ de $\mathbb{G}$. Ainda, $\mathbb{G}^{\prime}$ é um subgrafo de $\mathcal{F}$. Ademais, como existe a aresta $\left(c p \_r, c p \_p\right)$ então o vértice $c p \_p$ foi um vértice rotulado como $S O_{\mathcal{F}}$. Precisamos encontrar um vértice que estava acima de $c p \_p$ no caminho esquerdo da borda de $\mathcal{F}$, digamos $c p \_i n i$, de modo que $c p \_$ini era adjacente a $c p \_r$ em $\mathcal{F}$, assim, precisamos olhar os vértices adjacentes a $c p_{r}$ em $\mathbb{G}$. Pois, como $c p \_$ini pertence a $\mathbb{D}$, então a aresta $\left(c p \_i n i, c p \_r\right) \notin E\left(\mathbb{G}^{\prime}\right)$. Ainda, $c p \_$ini é um ancestral distante de $c p \_p$, pois, caso contrário, iria existir um triângulo que não face, contrariando a hipótese de que existe uma representação retangular de $\mathbb{G}$.

O problema deste caso é que existem alguns vértices que estão entre o caminho esquerdo da borda de $\mathbb{G}^{\prime}$ e a $\operatorname{aresta}\left(c p \_r, c p \_p\right)$. A figura 5.13 ilustra este caso. Seja $\mathbb{H}$ o grafo equivalente a região ilustrada na figura 5.13. Todas as arestas pontilhadas já foram processadas e não pertencem a $\mathbb{G}^{\prime}$.

Precisamos processar $\mathbb{H}$ de modo que garantimos $c p \_p$ não seja um ancestral distante de $c p \_r$. Para isso, vamos adicionar ao grafo $\mathbb{D}$ os caminhos que cobrem $\mathbb{H}$ iniciando pelo vértice $c p \_i n i$ e terminando no vértice $c p \_p$. Após isso, iremos remover o grafo $\mathbb{H}$ de $\mathbb{G}^{\prime}$. Ainda, vamos adicionar o vértice $c p_{\_} p$ como filho de $c p \_r$ e caso VerticeFinal $\mathbb{G}_{\mathbb{G}^{\prime}}$ for diferente de $N U L O$, iremos adicionar a aresta $\left(c p \_p\right.$, VerticeFinal $\left.\mathbb{G}_{\mathbb{G}^{\prime}}\right)$ a $\mathbb{D}$ e remover a aresta $\left(c p \_r, c p \_p\right)$ de $\mathbb{G}^{\prime}$. A figura 5.14 ilustra como o grafo $\mathbb{G}^{\prime}$ deve ficar após este processamento.

Voltando ao caso que estávamos tratando, os vértices $u$ e $v$ definem a componente pendente $\mathbb{H}$. Considere $c p \_p=v$ e $c p \_r=u$. Logo, podemos tratar $\mathbb{H}$ e prosseguir o processamento de $v$.

Outro caso é quando $u$ pertence a borda esquerda e $u$ não pertence a $\mathbb{D}$. Assim, $u$ não é o vértice $S O_{\mathbb{G}^{\prime}}$. A figura 5.15 ilustra este caso.

Iremos chamar a $\operatorname{aresta}(v, u)$ de desvio. Mais formalmente, uma desvio é uma aresta 


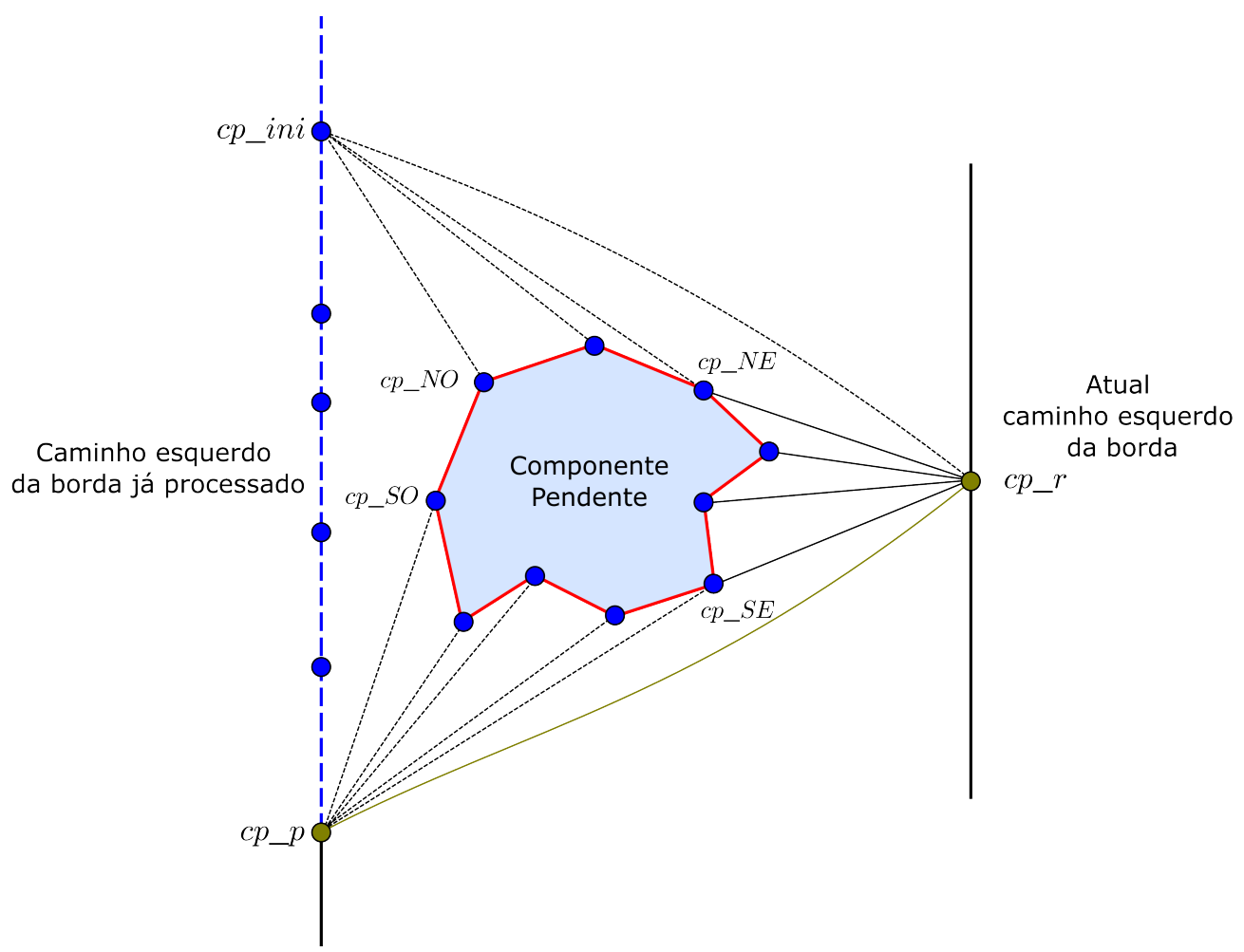

Figura 5.13: Componente pendente entre o atual caminho esquerdo da borda e o próximo caminho esquerdo da borda. [BS88]

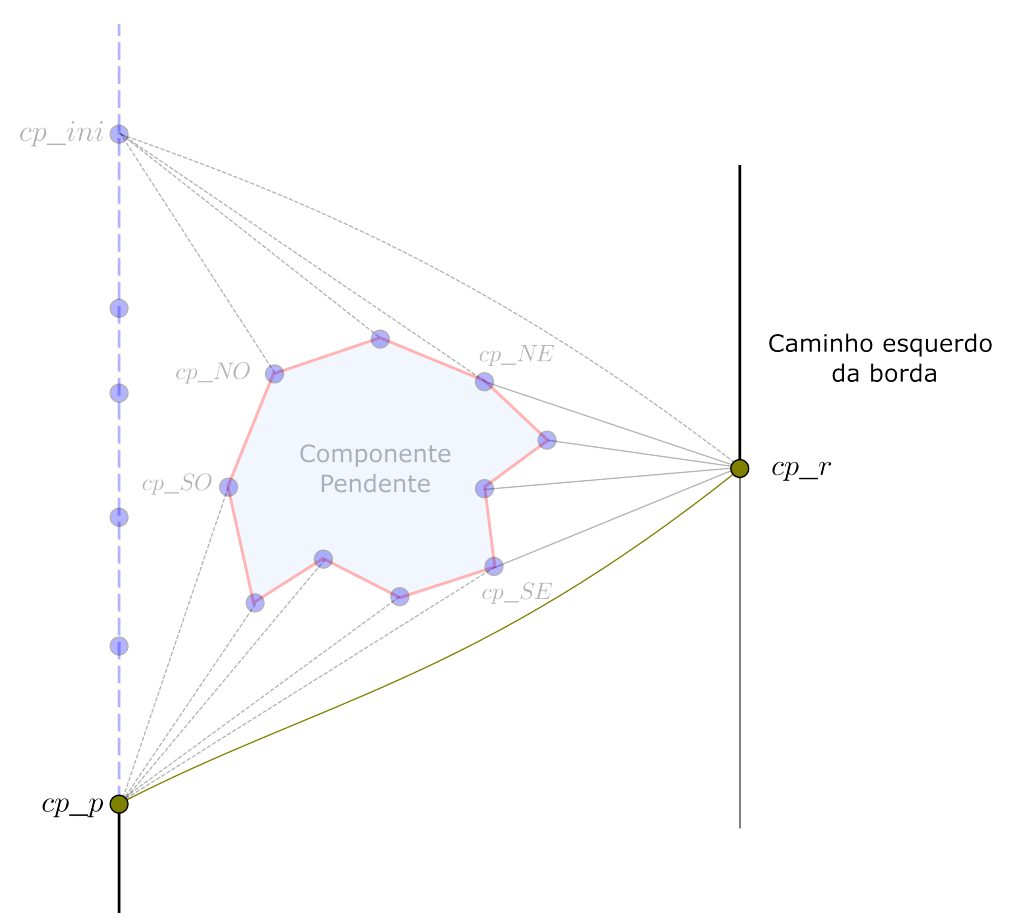

Figura 5.14: Grafo resultante após o processamento da componente pendente.

$(v, u)$ que satisfaz as seguintes propriedades:

P1. $v$ e $u$ pertencem ao atual caminho esquerdo da borda;

P2. $(v, u)$ não é uma aresta do atual caminho esquerdo da borda; e 


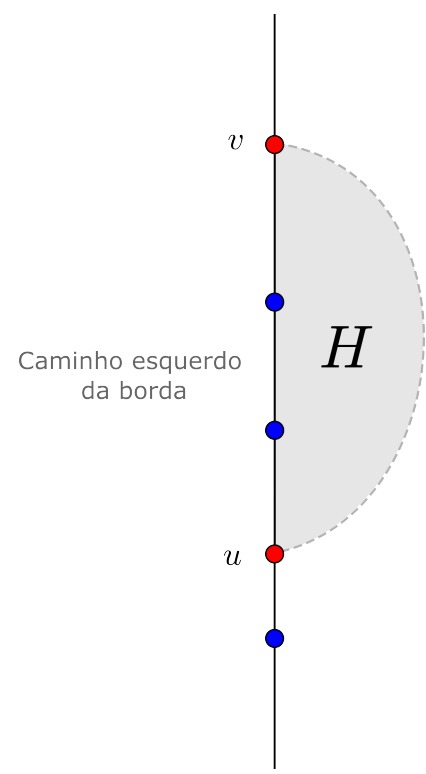

Figura 5.15: Ilustração de uma desvio no caminho esquerdo da borda de $\mathbb{G}^{\prime}$.

P3. $v$ é predecessor de $u$ no caminho esquerdo da borda e $u \notin V(\mathbb{H})$.

O problema de existir uma desvio é que se adicionarmos o caminho esquerdo a $\mathbb{H}$, temos que este caminho violará a condição de que para toda aresta $(v, u)$ de $\mathbb{G}, v$ não é um ancestral distante de $u$, nem $u$ de $v$.

Basicamente, se existir uma desvio $(v, u)$, precisamos garantir que $v$ não seja um ancestral distante de $u$, ou seja, em $\mathbb{D}$, ou $v$ é pai de $u$, ou não existe um caminho de comprimento maior que 1 de $v$ a $u$.

Considere as figuras 5.16A e 5.16B. A aresta $\left(c h \_p, c h \_r\right)$ é uma desvio de $\mathbb{G}^{\prime}$ de modo que $c h \_p$ é predecessor de $c h \_r$ no caminho esquerdo da borda de $\mathbb{G}^{\prime}$.

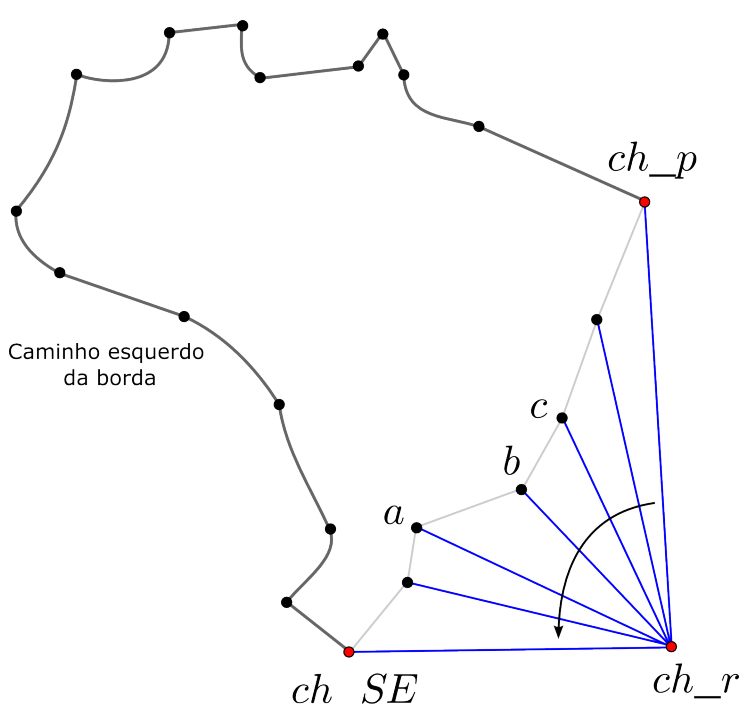

(A)

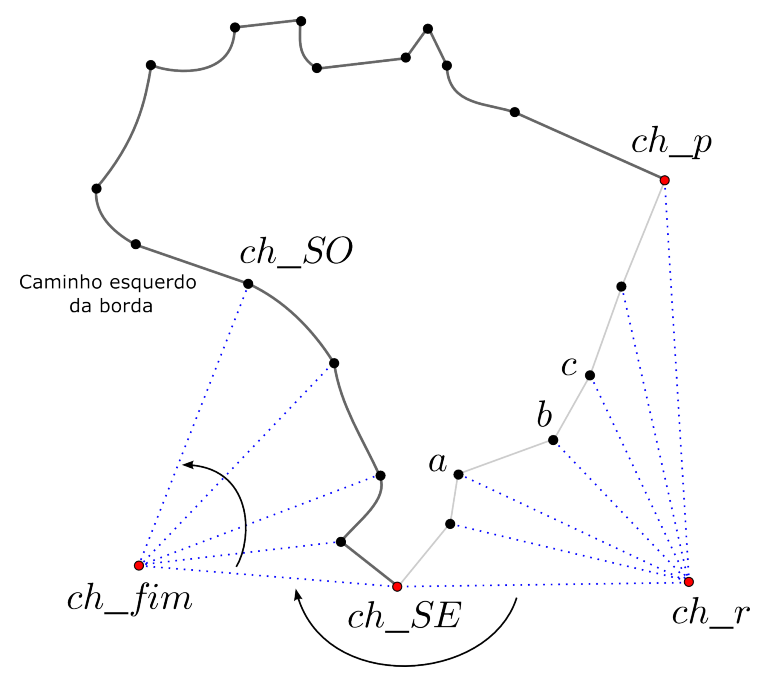

(B)

Figura 5.16: Ilustração de uma desvio $\left(c h \_p, c h \_r\right)$. [BS88] 
Considere o circuito $C=\left\langle c h \_p, \ldots, c h \_r, c h \_p\right\rangle$ formado pelo caminho esquerdo da borda mais a aresta $\left(c h \_p, c h \_r\right)$. Conforme a figura 5.16A ilustra podem existir alguns vértices internos ao circuito como, por exemplo, $a, b, c$, etc. Assim, a aresta $\left(c h \_p, c h \_r\right)$ será uma desvio para todos os caminhos esquerdos nesta região interna ao circuito $C$ (incluindo $C)$. Logo, precisamos resolver o problema não só para o atual circuito esquerdo da borda, mas para toda esta região.

Inicialmente, devemos isolar o subgrafo interno a $C$, digamos $\mathbb{H}$, de $\mathbb{G}^{\prime}$. Vamos mostrar como encontrar vértices que serão os cantos de $\mathbb{H}$.

Inicialmente, precisamos encontrar o vértice $c h \_S E$. Para isso, considere os vértices adjacentes a $c h \_r$ no sentido anti-horário começando pelo vértice $c h \_p$. O vértice $c h \_S E$ é o predecessor de $c h \_r$ no atual caminho esquerdo da borda. Note que $c h \_S E \neq c h \_p$, pois a $\operatorname{aresta}\left(c h \_p, c h \_r\right)$ não pertence ao caminho esquerdo da borda. Após encontrar $c h \_S E$, devemos remover de $\mathbb{G}^{\prime}$ as arestas que foram percorridas entre o vértice $c h \_p$ e $c h \_S E$, incluindo $\left(c h \_r, c h \_p\right)$ e $\left(c h \_r, c h \_S E\right)$.

Seja $c h \_f i m$ o primeiro vértice adjacente a $c h \_S E$ no sentido horário após o vértice $c h \_r$. Perceba que este vértice estará mais a esquerda do que o atual caminho esquerdo da borda. Pois, como existe uma representação retangular de $\mathbb{G}$ a escolha inicial dos rótulos $N O, N E, S O$ e $S E$ implicam que o primeiro caminho esquerdo da borda que foi construído não possui uma desvio. Logo, o atual caminho esquerdo do subgrafo não é o caminho esquerdo de $\mathbb{G}$. Assim, se percorrermos os vértices adjacentes a $c h \_S E$ no sentido horário, existe um vértice $\left(c h \_f i m\right)$ após $c h \_r$ e este está a esquerda de $c h \_S E$.

Agora, vamos percorrer a lista de adjacência de ch_fim no sentido anti-horário iniciando no vértice $c h \_S E$ e terminando no vértice $c h \_S O$, onde o vértice $c h \_S O$ é o último vértice que pertence ao caminho esquerdo e não pertence a $\mathbb{H}$. Isso é equivalente a andar no sentido contrário do caminho esquerdo da borda partindo de $c h \_S E$. Note que $c h \_S O \neq c h \_p$, pois $c h \_p \in V(\mathbb{H})$, ainda, $c h \_p$ não é adjacente a $c h \_f i m$. Entretanto, $c h \_S O$ pode ser o mesmo vértice que $c h \_S E$.

O grafo resultante $\mathbb{H}$ (figura 5.16B) está isolado de $\mathbb{G}^{\prime}$. Define-se os rótulos de $\mathbb{H}$ da seguinte forma: $N O_{\mathbb{H}}=N E_{\mathbb{H}}=c h \_p, S O_{\mathbb{H}}=c h \_S O$ e $S E_{\mathbb{H}}=c h \_S E$. Ademais, VerticeInicial $_{\mathbb{H}}=c h \_p$ e VerticeFinal $\mathbb{H}_{\mathbb{H}}=c h \_$fim.

Adicionando os caminhos que cobrem $\mathbb{H}$ a $\mathbb{D}$ processamos a região entre o caminho esquerdo da borda e a desvio. De modo que $\left(c h \_p, c h \_r\right)$ não viola o lema 5.2.

Agora que tratamos todos os casos de $i$. Vamos tratar um único caso para $i$.

Neste caso, $v$ está "abaixo" de $u$. A figura 5.17 ilustra este caso.

Para encontrar a componente pendente $\mathbb{H}$, vamos considerar $u=c p \_r$ e $v=c p \_p$. Após 


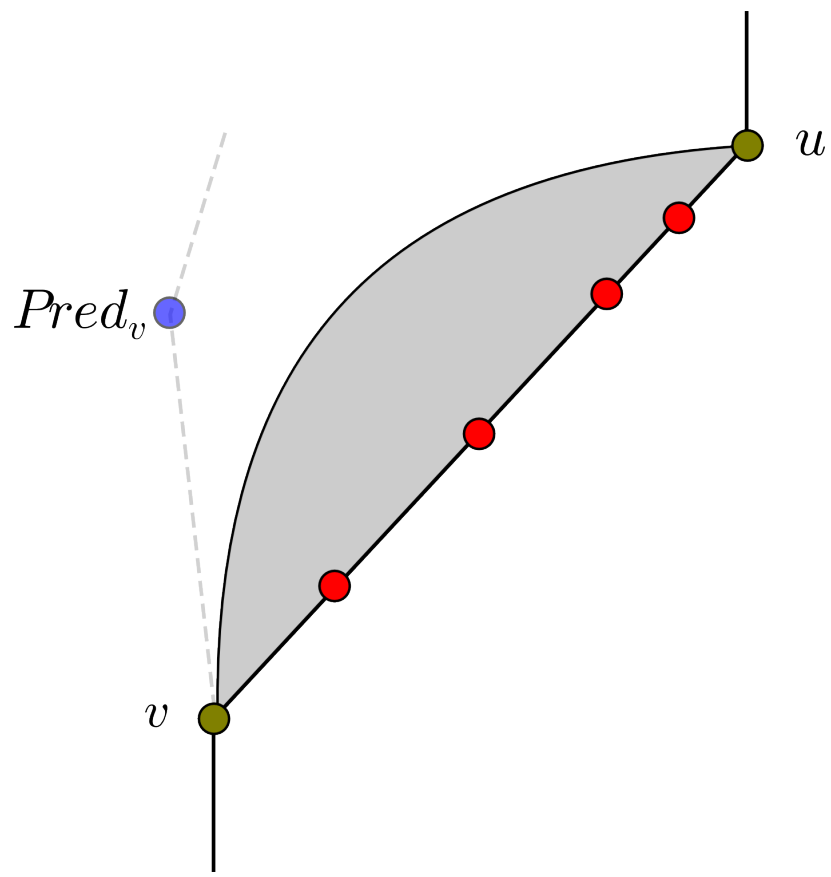

Figura 5.17: Ilustração da componente pendente acima de $v$ em $\mathbb{G}^{\prime}$.

encontrar $\mathbb{H}$, vamos processá-la conforme apresentado anteriormente. Concluindo assim, os casos de quando $v$ não pertence à borda direita.

Suponha agora que $v$ pertence a borda direita de $\mathbb{G}^{\prime}$.

Se $v$ não é o primeiro vértice do caminho esquerdo da borda, então pred $_{v}$ é diferente de $N U L O$. Se $\operatorname{pred}_{v}$ não pertence à borda direita de $\mathbb{G}^{\prime}$, então existe uma região acima de $v$ que deve ser processada, de modo que o retângulo correspondente a $v$ esteja abaixo (não necessariamente imediatamente abaixo) de todos os retângulos correspondentes aos vértices acima de $v$. A figura 5.18A ilustra este caso.

Considere $\mathbb{H}$ como sendo o grafo equivalente a "região acima de $v$ " ilustrada na figura 5.18A. Os cantos de $\mathbb{H}$ serão: $N O_{\mathbb{H}}=N O_{\text {prox }}, S O_{\mathbb{H}}=S E_{\mathbb{H}}=v$ e $N E_{\mathbb{H}}=N E_{\mathbb{G}^{\prime}}$. Ainda, VerticeInicial $l_{\mathbb{H}}=$ VerticeInicial $_{\mathbb{G}^{\prime}}$.

Perceba que $v$ é uma articulação de $\mathbb{G}^{\prime}$, logo, quando formos processar o grafo $\mathbb{H}$, precisamos isolar $v$ da parte inferior do grafo.

Depois de processar $\mathbb{H}$ e adicionar os caminhos a $\mathbb{D}$ de forma apropriada, devemos remover $\mathbb{H}-v$ de $\mathbb{G}^{\prime}$. Note que agora precisamos processar os vértices que devem estar "abaixo" de $v$. Podemos considerar que $v$ inicia o caminho esquerdo da borda de $\mathbb{G}^{\prime}$. (Figura 5.18B)

Note que $v$ pode ser adjacente a um vértice $u$ que não é o próximo vértice do caminho esquerdo de $v$. Caso exista tal vértice $u$, temos dois casos que devem ser tratados: $(i)$ se $u$ não pertence a $\mathbb{D}$; e $(i i)$ se $u$ pertence a $\mathbb{D}$.

O primeiro caso é quando a aresta $(v, u)$ é uma desvio de $\mathbb{G}^{\prime}$. Logo, iremos tratar conforme 


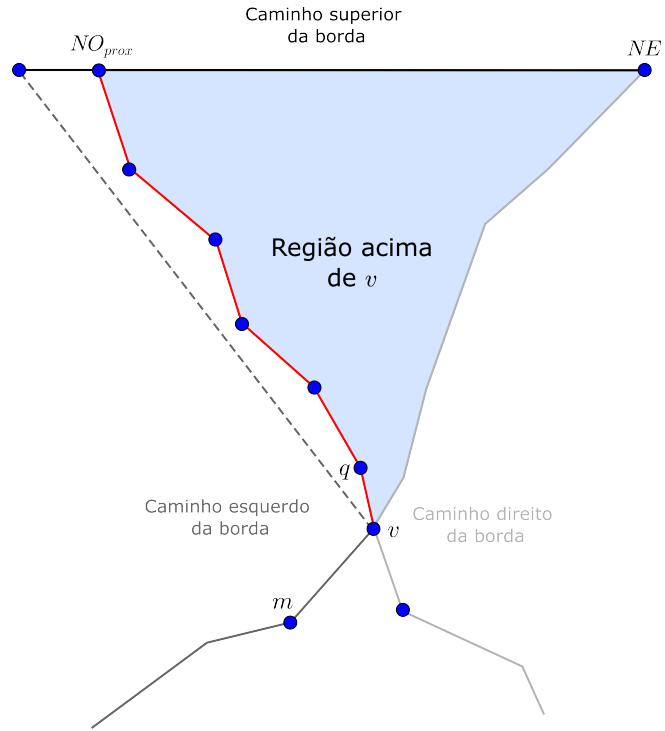

(A)

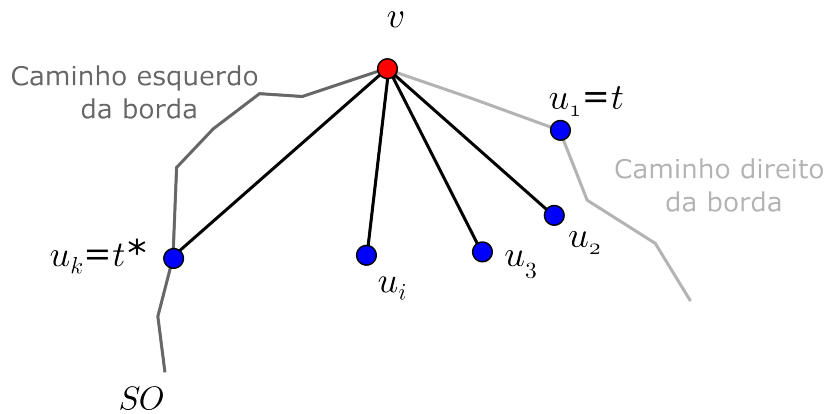

(B)

Figura 5.18: Regiões que devem ser processadas por PROCESSAR-VÉRTICE-BORDA-DiREITA. (A) Região acima de p. (B) Região entre o caminho esquerdo da borda e pt.[BS88]

apresentado anteriormente.

Para o caso $i i$, onde $u$ pertence a $\mathbb{D}$. Neste caso, $u$ deve ser igual a $S O$, pois os únicos vértices que podem restar após o processamento de um caminho esquerdo são os vértices $N O_{\mathbb{G}^{\prime}}$ e $S O_{\mathbb{G}^{\prime}}$. Como $u$ está abaixo de $v$, então $u=N O_{\mathbb{G}^{\prime}}$.

Este caso é semelhante ao caso da componente pendente que foi tratado anteriormente. Se considerarmos $c p \_p=u$ e $c p \_r=v$ podemos obter o grafo $\mathbb{H}$ de modo similar e tratar a aresta $(v, u)$. Finalizando os casos para quando $v$ pertence a borda direita de $\mathbb{G}^{\prime}$.

Acreditamos que a implementação do algoritmo para encontrar um grafo dos caminhos dirigidos não é trivial. Ainda, existem muitos detalhes para que o algoritmo resultante seja linear. Assim, iremos apresentar de forma detalhada no Apêndice A.

\subsection{Algoritmo De Bhasker E Shani}

Agora que já apresentamos como obter um grafo dos caminhos dirigidos $\mathbb{D}$ de um grafo plano triangulado $\mathbb{G}$, vamos mostrar o algoritmo desenvolvido por Bhasker e Shani que encontra uma representação retangular de $\mathbb{G}$ utilizando $\mathbb{D}$.

Como já dito anteriormente, se analisarmos a figura 5.1C, podemos dividir a representação retangular em colunas de modo que em uma coluna, as únicas arestas verticais devem estar à esquerda ou à direita. É como se em cada segmento de reta vertical, cortássemos o desenho de modo que este segmento divida o plano em dois (linhas pontilhadas da figura 5.1C). Assim, após cortar o desenho em cada reta vertical, teríamos 
regiões limitadas por dois cortes verticais, vamos chamar estas regiões de colunas. A ideia do algoritmo de Bhasker e Shani é construir a representação retangular a partir destas colunas. Ele utiliza o grafo dos caminhos dirigidos para, a partir da coluna mais a esquerda, posicionar os retângulos de modo que satisfaça as restrições definidas pelas arestas de $\mathbb{G}$. Assim que concluída uma coluna, começa a ser construído a próxima coluna a direita, e assim sucessivamente.

O algoritmo de Bhasker e Shani tem como objetivo definir as coordenadas cartesianas das retas que limitam cada retângulo da representação retangular de $\mathbb{G}$. Em seu algoritmo, as coordenadas das retas são números reais. Entretanto, é fácil fazer um algoritmo para converter as coordenadas para valores inteiros. Ainda, vamos considerar que a origem é o canto superior esquerdo e a coordenada $y$ é maior quando vamos para baixo. Ainda, observe que para os lados verticais de um retângulo a coordenada $y$ não importa, pois estes lados são limitados pelas coordenadas y dos lados horizontais. De modo semelhante, só precisamos definir as coordenadas $y$ dos lados horizontais. Assim, para cada vértice $v$ do grafo $\mathbb{G}$ temos as seguintes variáveis associadas a ele:

visitado: É uma variável booleana que indica se o vértice $v$ já foi visitado anteriormente pelo algoritmo;

esq: Uma variável inteira para armazenar a coordenada $x$ da reta vertical esquerda do retângulo associado a $v$;

baixo: Uma variável ponto flutuante para armazenar a coordenada $y$ da reta horizontal de baixo do retângulo associado a $v$;

cima: Uma variável ponto flutuante para armazenar a coordenada $y$ da reta horizontal de cima do retângulo associado a $v$;

A figura 5.19 ilustra este sistema de coordenadas.

O algoritmo para encontrar uma representação retangular consiste em duas funções: Construir-Representação-Retangular e Posicionar-Retângulo. A função ConstruiR-REPRESEnTAÇÃo-RETAngular inicializa algumas variáveis e chama a função Posicionar-Retângulo para o vértice $v_{\infty}$. Ainda, a função ConstruirREPRESENTAÇÃO-RETANGULAR tem como objetivo definir a coordenada $x$ das arestas verticais direitas de todos os vértices que estão no caminho mais direito de $\mathbb{D}$. A linha 5 garante que iremos definir todas as coordenadas dos vértice de $\mathbb{D}-v_{\infty}$.

Vamos abusar um pouco e definiremos que a saída da função ConstruirREPRESENTAÇÃO-RETANGULAR é uma representação retangular, porém, o que o algoritmo faz é definir as coordenadas cartesianas das retas que limitam cada retângulo da 

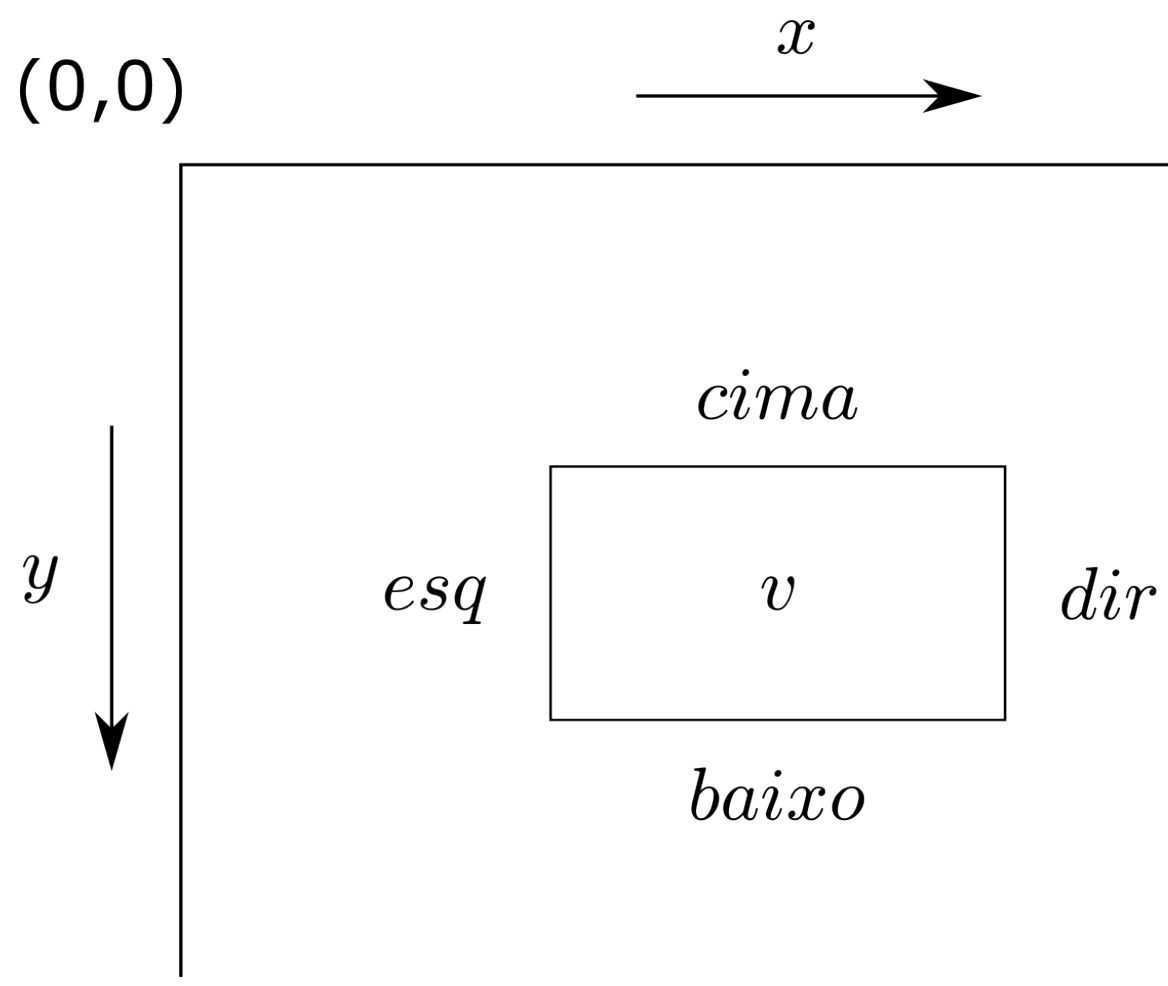

Figura 5.19: Sistema de coordenadas utilizado para representar a representação retangular. [BS88]

representação retangular. Entretanto, a representação retangular pode ser facilmente obtida pelas variáveis esq, dir, cima e baixo associada a cada vértice de $\mathbb{D}-v_{\infty}$;

As variáveis globais $x, y, y_{\max }$ e PrimeiroCaminho são inicializadas em ConstruirREPRESENTAÇÃO-RETANGUlar. A variável $x$ armazena o valor da coordenada $x$ da coluna em que os retângulos estão sendo posicionados. A variável y armazena a coordenada do valor de v.baixo, onde $v$ é o último retângulo que foi posicionado na coluna atual. De modo equivalente, $y$ armazena a coordenada $y$ da aresta de cima do próximo retângulo que será adicionado na coluna atual.

Como não modificamos os grafos $\mathbb{G}$ e $\mathbb{D}$, também vamos considerá-los como variáveis globais.

Algoritmo Construir-RepresentaÇão-Retangular( $\mathbb{G}, \mathbb{D})$

ENTRADA: Grafo plano triangulado $\mathbb{G}$ e grafo dos caminhos dirigidos $\mathbb{D}$ de $\mathbb{G}$.

SAÍDA: Representação retangular do grafo $\mathbb{G}$ utilizando $\mathbb{D}$.

1. $x \leftarrow y \leftarrow 0$;

2. PrimeiroCaminho $\leftarrow$ Verdadeiro;

3. $\quad$ visitado $\leftarrow$ Falso, $\forall v \in V(\mathbb{D})$;

4. $\quad v_{\infty} . e s q \leftarrow v_{\infty}$. dir $\leftarrow v_{\infty}$. cima $\leftarrow v_{\infty}$.baixo $\leftarrow 0$;

5. Posicionar-Retângulo $\left(v_{\infty}\right)$;

6. Para cada vértice $v$ que pertence ao caminho mais a direita de $\mathbb{D}$ faça

7. $\quad$ v.dir $\leftarrow x+1$; 
A seguir, apresentamos a função recursiva Posicionar-RetÂngulo. Esta função tem como objetivo encontrar as coordenadas de $v$ e todos os vértices que são descendente do vértice $v$ no dígrafo $\mathbb{D}$.

\section{Algoritmo Posicionar-RetÂngulo $(v)$}

ENTRADA: Vértice $v \in V(\mathbb{D})$.

SAÍDA: As coordenadas cartesianas das retas que limitam todos os retângulos correspondente ao dígrafo enraizado em $v$.

1. Se $v \neq v_{\infty}$ então

2. $\quad$ v.visitado $\leftarrow$ Verdadeiro;

3. $\quad$ v.esq $\leftarrow x$;

4. $\quad$ v.cima $\leftarrow y$;

5. Se PrimeiroCaminho $=$ Verdadeiro então

6. $y \leftarrow y+1$;

7. $\quad$ v.baixo $\leftarrow y$;

8. $\quad y_{\max } \leftarrow y$;

9. Senão

10. $\quad$ v.baixo $\leftarrow y$;

11. Para todo vértice $u$ que é adjacente a $v$ em $\mathbb{G}$ faça

12. Se u.visitado $=$ Verdadeiro e $u$.baixo $\neq$ v.top então

13. $\quad / *$ está a esquerda de $v^{*} /$

14. $\quad$ u.dir $\leftarrow x$;

15. Se $\quad$ S.baixo $>$ v.baixo então

16. $\quad y \leftarrow(u . b a i x o+\max \{$ u.cima, v.cima $\}) / 2$;

$17 . \quad$ v.baixo $\leftarrow$;

18. Se $v$ não possui filhos em $\mathbb{D}$ então

19. $\quad$ v.baixo $\leftarrow y_{\max }$;

20. Senão

21. PrimeiroFilho $\leftarrow$ Verdadeiro; /* Variável local */

22. Para todo filho $w$ de $v$ em $\mathbb{D}$ faça /* Percorrer da esquerda para direita */

23. Se w.visitado $=$ Verdadeiro então

24. $\quad$ v.baixo $\leftarrow$ w.cima;

25. Senão Se PrimeiroFiho $=$ Verdadeiro então

26. PrimeiroFilho $\leftarrow$ Falso;

27. Posicionar-RetÂngulo $(w)$;

$28 . \quad$ Senão

29. $\quad$ PrimeiroCaminho $\leftarrow$ Falso;

30. $\quad x \leftarrow x+1$;

31. $\quad y \leftarrow$ v.baixo;

32. Posicionar-Retângulo $(w)$; 
Quando estamos executando Posicionar-RetÂngulo, temos que v.esq deve ser igual a $x$ e v.cima deve ser igual a $y$. Ainda, na linha 1 temos que v.visitado $=$ Falso e com exceção de quando $v=v_{\infty}, v$ sempre é um vértice de $\mathbb{D}$ cujo retângulo deve ser posicionado na representação retangular. Os retângulos são posicionados nas linhas 1-32.

Se o vértice $v$ pertence ao primeiro caminho, ou seja, o caminho mais a esquerda de $\mathbb{D}$, então a altura do retângulo associado a $v$ é igual a 1 unidade. O último retângulo irá possuir v.baixo $=y_{\max }($ linha 8$)$.

Caso $v$ não pertença ao primeiro caminho, então v.baixo é determinado pelos retângulos que são adjacentes a $v$ e estão à sua esquerda.

Entretanto, se percorrermos a lista de adjacência do vértice $v$ existem quatro tipos de vértices:

A: Vértices que estão à direita de $v$. Estes vértices possuem suas variáveis visitado igual a Falso;

B: Existe no máximo um único vértice $u$ que está acima de $v$ na coluna que estamos construindo. Observe que pela definição de coluna, dentro de uma coluna não existe uma aresta vertical. Se $v$ é o primeiro retângulo da coluna ( $v$ é filho de $v_{\infty}$ ), então não existe tal $u$. Caso exista tal $u$, então u.visitado $=$ Verdadeiro e $u$.baixo $=$ v.cima;

C: Existe no máximo um único vértice $u$ que está abaixo de $v$ coluna que estamos construindo. Este vértice $u$ é filho de $v$ em $\mathbb{D}$;

D: Todos os outros vértices adjacentes a $v$. Estes vértices possuem suas variáveis visitado $=$ Verdadeiro e correspondem a um conjunto de retângulos adjacentes na coluna anterior $(x-1)$.

Para determinar a variável baixo do vértice $v$, precisamos analisar todos os vértices do tipo C e D.

Vamos mostrar a seguir que é possível existir um vértice pertencente a $\mathrm{C}$ e já ter sido visitado. Seja $u$ é um vértice que pertence a categoria C. Caso $u$ já tenha sido visitado, então já sabemos qual é a coordenadas de todas as retas que limitam o retângulo associado a $u$, logo, se $u$ pertence à categoria $\mathrm{C}$, temos que u.cima $=$ v.baixo. Este caso é ilustrado na figura 5.20A.

Se não existe um vértice pertencente a $\mathrm{C}$, ou para todos os vértices $u$ que são adjacentes a $v$ temos que u.visitado $=$ Falso, então v.baixo é determinado pelos vértices pertencentes à categoria D (Figura 5.20B).

Para qualquer vértice $u$ da categoria $\mathrm{D}$ temos que $u$.dir $=x$, pois $u$.dir $=v . e s q$. Note que $u$.visitado $=$ Verdadeiro e $u$.baixo $\neq$ v.cima se e somente se, $u \in \mathrm{D}$ ou $u \in \mathrm{C}$ e $u$ se 


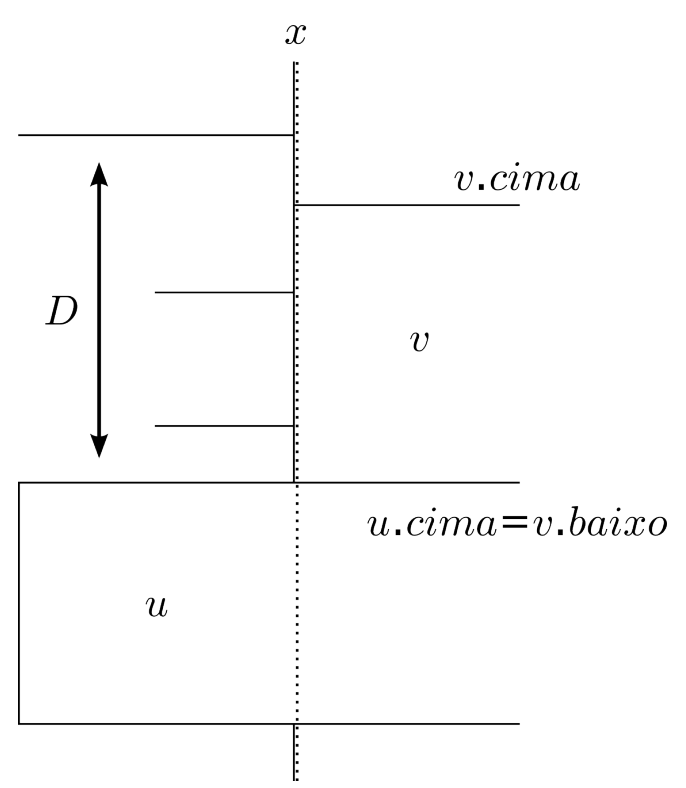

(A)

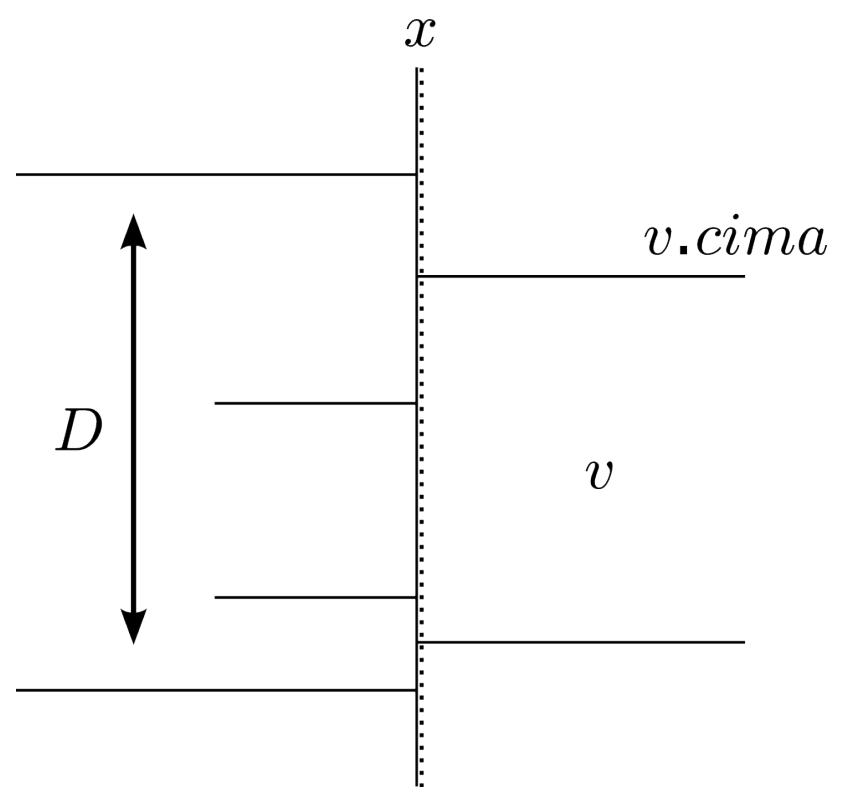

(B)

Figura 5.20: Casos para determinar a variável v.baixo.[BS88]

enquadra no caso retratado na figura 5.20A. As linhas 14-17 não consideram que se o vértice não se enquadra no caso da figura 5.20A. Assim, sob esta suposição, devemos executar estas linhas somente para quando $u \in \mathrm{D}$. Depois de atribuirmos estes valores às variáveis dir dos vértices $u$, devemos definir as variáveis baixo de modo que se existe um filho $w$ de $v$ que deve ser adjacente a algum vértice $u$, esta restrição possa ser satisfeita. Se existe tal retângulo $w$, então $w$.visitado $=$ Falso, e $w$ deve ser adjacente a algum retângulo $u \in$ D. É fácil ver que $w$ deve ser adjacente ao retângulo $u$ que está mais abaixo do conjunto de retângulos pertencente a D (linhas 16 e 17).

Caso contrário, $u$ deve se enquadrar no caso da figura 5.20A, então as linhas 14-17 só irão atribuir o valor $u$.dir $=x$ para todos os vértices $u \in \mathrm{D}$.

Para completar o posicionamento do retângulo relativo ao vértice $v$. Temos as linhas 18-32. Estas linhas tratam os casos se existem algum filho de $v$ que ainda não posicionado, o caso da figura $5.20 \mathrm{~A}$ e o caso que C é vazio.

Se C for vazio, então $v$ não possui filho. Assim, v é o último retângulo da coluna que estamos processando, logo, devemos estender $v$ até $y_{\max }$ (linha 19).

Caso contrário, $v$ possui ao menos um filho. Precisamos percorrer a lista de adjacência de $v$ da esquerda para direita, ou seja, percorrer as arestas que estão saindo de $v$ no sentido anti-horário. Seja $w$ um filho de $v$. Considere os casos:

- $w$.visitado $=$ Verdadeiro: Este é o caso representado na figura 5.20A com $w=u$. Neste caso, precisamos definir v.baixo $=$ w.cima; 
- $w$ é o filho mais a esquerda de $v$ : Neste caso, só precisamos chamar recursivamente a função PosicionAR-RETÂNGUlo para $w$;

- $w . v i s i t a d o=$ Falso e $w$ não é o filho mais a esquerda de $v$ : Neste caso, $w$ deve ser posicionado na próxima coluna. Então, $x$ será incrementado e $w$ e seus descendentes serão posicionados pela chamada recursiva da função Posicionar-REtÂngulo. Observe que como y é uma variável global, ela pode ser modificada na chamada recursiva das linhas 27 ou 32 em outras iterações do laço da linha 22, então é necessário inicializar $y$ antes de cada chamada da função PosicIONAR-REtÂNGULO.

É fácil ver que o algoritmo para encontrar uma representação retangular a partir de um grafo dos caminhos dirigidos está correto, pois este trata todos os casos que podem existir quando estamos posicionando um retângulo na representação retangular.

Ainda, como cada vértice só é visitado uma única vez, podemos concluir que este algoritmo possui complexidade linear na quantidade de vértices de $\mathbb{G}$.

Por fim, apresentamos no apêndice A os detalhes de implementação do algoritmo deste capítulo. 



\section{6}

Algoritmo DE He

O algoritmo que apresentamos nesta seção foi desenvolvido por He em [He93]. O algoritmo é baseado na caracterização de Koźmiński e Kinnen apresentada no capítulo 4, consistindo em encontrar uma determinada rotulação das arestas do grafo da entrada e a partir desta construir dois grafos dirigidos. Nestes grafos dirigidos, iremos encontrar uma representação retangular a partir dos $s, t$-caminhos orientados.

\subsection{RotulaÇÃo REgular DAS ARESTAS DE UM GRAFO PLANO TRIANGULADO}

Seja $\mathbb{H}$ um grafo plano triangulado que é entrada do problema da representação retangular. A partir de agora, suponha que o grafo $\mathbb{H}$ possui uma representação retangular.

Para o algoritmo de He, precisamos modificar o grafo $\mathbb{H}$. Iremos utilizar o conceito de 4-extensão que foi introduzido no capítulo 4. Entretanto, para encontrar uma 4-extensão do grafo $\mathbb{H}$ precisamos rotular 4 vértices que incidem na face externa de $\mathbb{H}\left(v_{N O}, v_{N E}, v_{S E}, v_{S O}\right)$ para serem os cantos da representação retangular. Como existe uma representação retangular de $\mathbb{H}$, podemos assumir que existe uma escolha dos 4 rótulos que torna viável a construção de uma 4-extensão de $\mathbb{H}$. Ademais, suponha que sabemos quais vértices devemos rotular.

Ainda, como já mostrado no capítulo 4, podemos calcular todas as possibilidades de 
cantos em tempo polinomial.

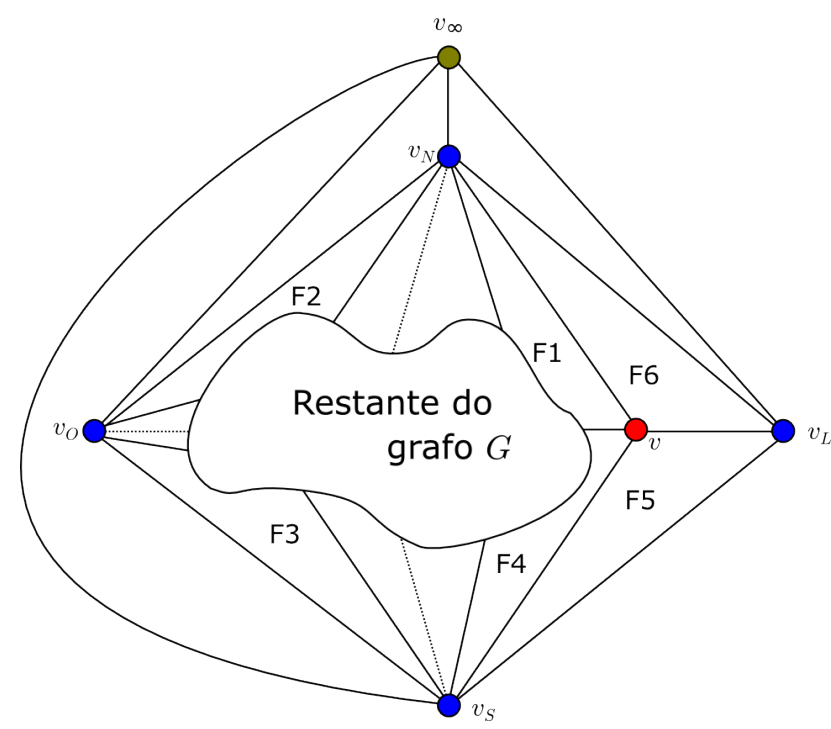

Figura 6.1: Exemplo de uma 4-extensão $\mathbb{E}$ de $\mathbb{H}$.

Considere $\mathbb{E}$ como sendo uma 4-extensão de $\mathbb{G}$ (figura 6.1 ). Observe o vértice $v_{\infty}$ desta figura. Pela construção, $v_{\infty}$ possui exatamente 4 vizinhos: $v_{N}, v_{S}, v_{O}$ e $v_{L}$. Ademais, se removermos o vértice $v_{\infty}$, o circuito $\left\langle v_{N}, v_{L}, v_{S}, v_{O}, v_{N}\right\rangle$ define a face externa de $\mathbb{E}-v_{\infty}$.

A partir de agora, vamos considerar como entrada do nosso problema o grafo $\mathbb{G}=\mathbb{E}-v_{\infty}$, onde $\mathbb{E}$ é uma 4-extensão do grafo plano triangulado $\mathbb{H}$. Assim, neste capítulo, sempre que nos referirmos ao grafo $\mathbb{G}$, estamos considerando que existe um grafo plano triangulado $\mathbb{H}$ que satisfaz o teorema 4.5 .

Definição 6.1 (Rotulação regular das arestas): Uma rotulação regular das arestas do grafo $\mathbb{G}$ é uma partição das arestas que não incidem na face externa em dois subconjuntos $\left\{T_{1}, T_{2}\right\}$ de arestas direcionadas de modo que satisfaça as seguintes propriedades:

1. Para cada vértice $v$ que não pertence à face externa de $\mathbb{G}$, as arestas de $\delta(v)$ possuem, na ordem horária, os seguintes rótulos: um conjunto não vazio de arestas saindo de $v$ pertencentes a $T_{1}$; um conjunto não vazio de arestas saindo de $v$ pertencentes a $T_{2}$; um conjunto não vazio de arestas entrando em $v$ pertencentes a $T_{1}$; um conjunto não vazio de arestas entrando em $v$ pertencentes a $T_{2}$.

2. Todas as arestas que incidem em $v_{N}$ que não incidem na face externa pertencem a $T_{1}$ e estão entrando em $v_{N}$; Todas as arestas que incidem em $v_{S}$ que não incidem na face externa pertencem a $T_{1}$ e estão saindo de $v_{S}$; Todas as arestas que incidem em $v_{O}$ que não incidem na face externa pertencem a $T_{2}$ e estão saindo de $v_{O}$. Todas as arestas que incidem em $v_{L}$ que não incidem na face externa pertencem a $T_{2}$ e estão entrando em $v_{L}$. 
A figura 6.2 ilustra a segunda propriedade da definição 6.1 .

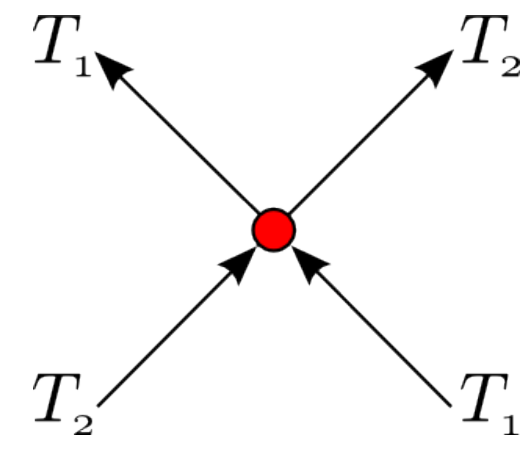

Figura 6.2: Segunda propriedade da definição 6.1.

De modo semelhante ao apresentado no capítulo 5, vamos orientar as arestas do grafo $\mathbb{G}$ particionando-as em dois conjuntos $T_{1}$ e $T_{2}$. A interpretação para um arco $u v \in T_{1}$ é que o retângulo correspondente a $u$ está embaixo do retângulo correspondente a $v$. Para um arco $u v \in T_{2}$, dizemos que o retângulo correspondente a $u$ está a esquerda do retângulo correspondente a $v$. As arestas incidentes a $v_{\infty}$ não são direcionadas. É fácil ver que em $T_{1}$, todas as arestas incidentes a $v_{N}$ deve ser orientada entrando em $v_{N}$ e as que incidem em $v_{S}$ devem ser orientadas saindo do vértice. De modo similar, em $T_{2}$, todas as arestas incidentes a $v_{O}$

Assim, se existe uma representação retangular de $\mathbb{G}$, então existe uma rotulação regular das arestas de $\mathbb{G}$, de modo que $T_{1}$ representa a relação de "abaixo de" e "acima de" entre os vértices de $\mathbb{G}$. De modo similar, $T_{2}$ representa a relação de "à esquerda de" e "à direita de".

Antes de apresentar o algoritmo que constrói uma representação retangular a partir do grafo $\mathbb{G}$, vamos provar algumas propriedades de uma rotulação regular das arestas.

Seja $\mathbb{G}$ um grafo plano triangulado e $\left\{T_{1}, T_{2}\right\}$ uma rotulação regular das arestas. Considere $\mathbb{G}_{1}$ como sendo o subgrafo orientado de $\mathbb{G}$ induzido pelas arestas que pertencem a $T_{1}$ mais as seguintes arestas orientadas: $v_{S} v_{O}, v_{O} v_{N}, v_{S} v_{L}$ e $v_{L} v_{N}$. Considere $\mathbb{G}_{2}$ como sendo o subgrafo orientado de $\mathbb{G}$ induzido pelas arestas que pertencem a $T_{2}$ mais as seguintes arestas orientadas: $v_{O} v_{S}, v_{S} v_{L}, v_{O} v_{N}$ e $v_{N} v_{L}$. Vamos chamar $\mathbb{G}_{1}$ de $S, N$-rede e $\mathbb{G}_{2}$ de $O, L$-rede de $\mathbb{G}$ a partir de $\left\{T_{1}, T_{2}\right\}$. As figuras $6.4 \mathrm{~A}$ e $6.4 \mathrm{~B}$ ilustram, respectivamente, a $S, N$-rede e a $O, L$-rede do grafo ilustrado na figura 6.3. 


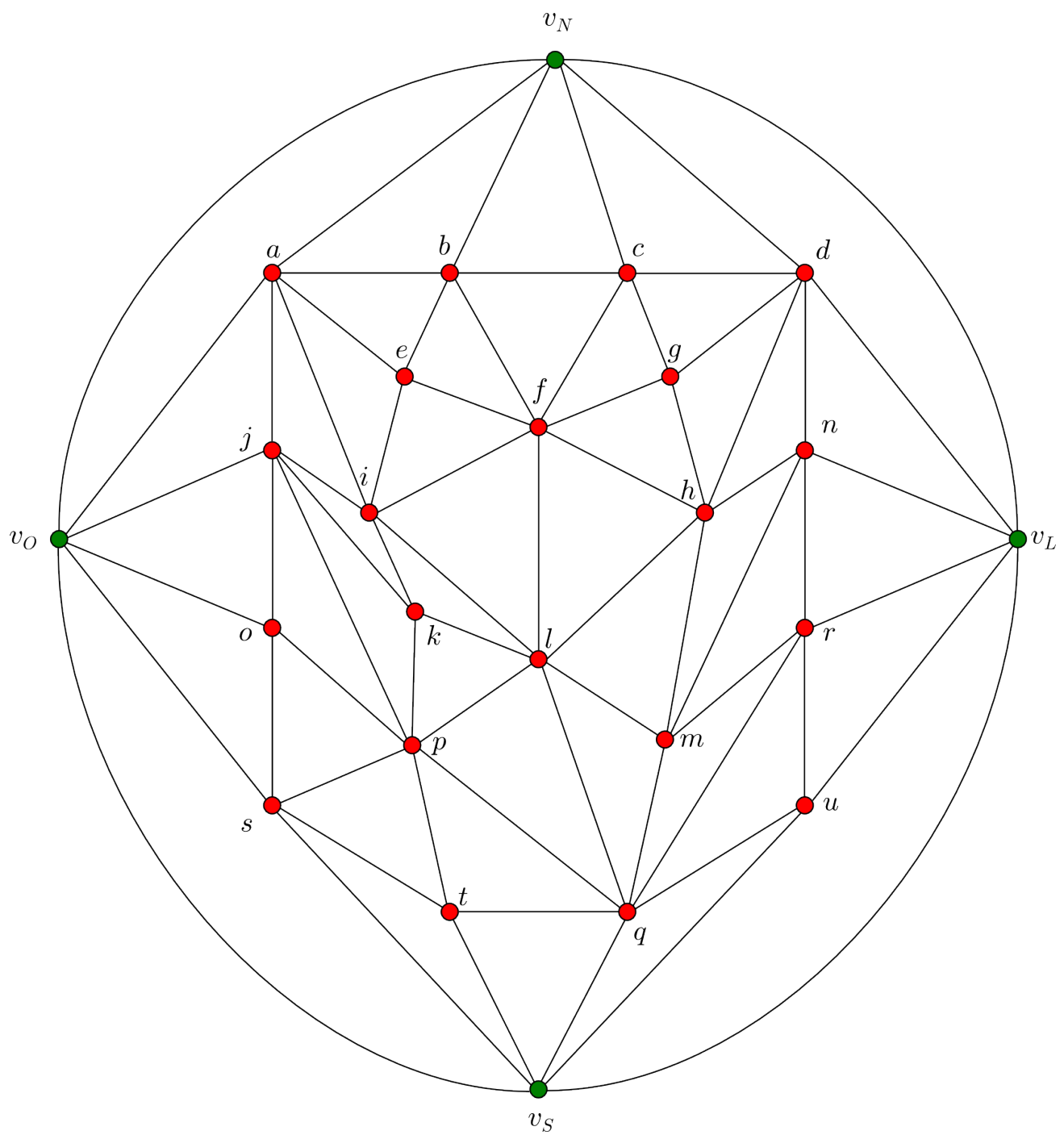

Figura 6.3: Exemplo de um grafo plano triangulado conveniente. 


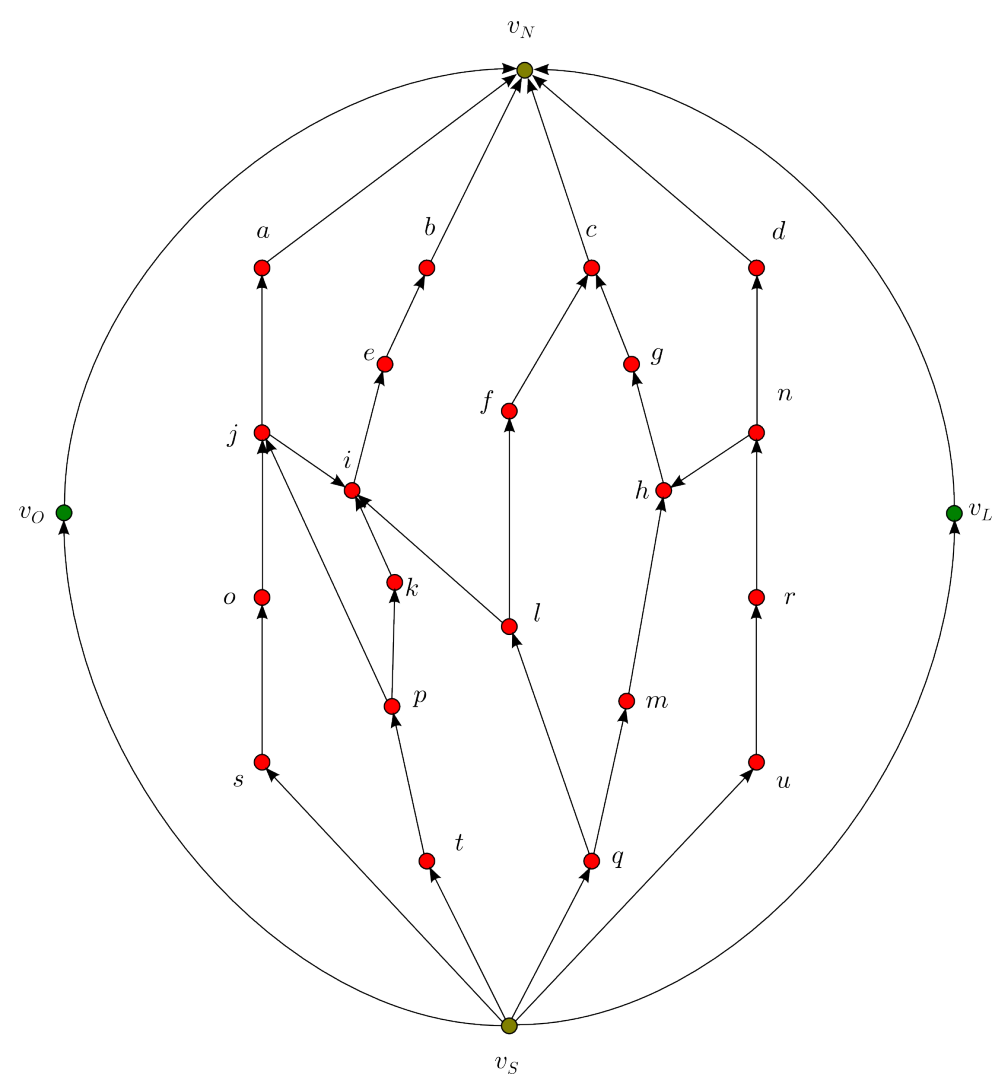

(A)

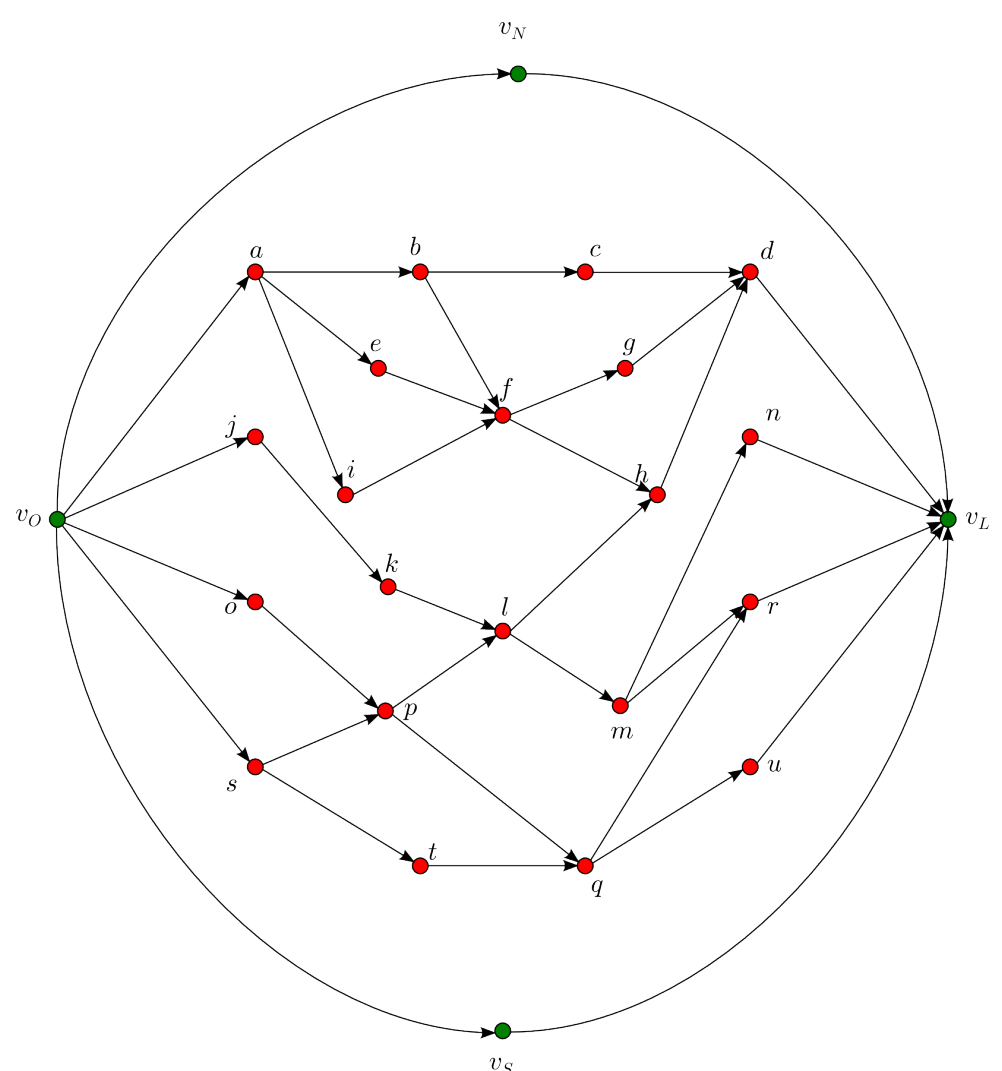

(B)

Figura 6.4: (A) Ilustração de uma $S, N$-rede do grafo ilustrado na figura 6.3 a partir de uma rotulação regular das arestas $\left\{T_{1}, T_{2}\right\}$. (B) Ilustração de uma $O, L$-rede do grafo ilustrado na figura 6.3 a partir de uma rotulação regular das arestas $\left\{T_{1}, T_{2}\right\}$ 
Em um grafo orientado, dizemos que um vértice $v$ é uma fonte se o grau de entrada de $v$ é igual a 0 . Dizemos que $v$ é um sorvedouro se o grau de saída de $v$ é igual a 0.

Lema 6.1: Seja $\mathcal{F}$ um grafo plano triangulado. Considere $\mathbb{G}=\mathbb{H}-v_{\infty}$, onde $\mathbb{H}$ é a 4-extensão de $\mathcal{F}$. Seja $\left\{T_{1}, T_{2}\right\}$ uma rotulação regular das arestas de $\mathbb{G}$. Seja $\mathbb{G}_{1}$ uma $S, N$-rede e $\mathbb{G}_{2}$ uma $O, L$-rede de $\mathbb{G}$ a partir de $\left\{T_{1}, T_{2}\right\}$. Então as seguintes propriedades são válidas:

1. $\mathbb{G}_{1}$ é acíclico com $v_{S}$ sendo a única fonte e $v_{N}$ o único sorvedouro;

2. $\mathbb{G}_{2}$ é acíclico com $v_{O}$ sendo a única fonte e $v_{L}$ o único sorvedouro;

Demonstração: Vamos fazer a prova para o item 1. O item 2 pode ser provado de modo análogo.

Primeiramente, vamos mostrar que $\mathbb{G}_{1}$ é acíclico.

Suponha, por absurdo, que existe um circuito $\left\langle v_{1}, v_{2}, \ldots, v_{k}, v_{1}\right\rangle$ em $\mathbb{G}_{1}$. Pela definição de $\mathbb{G}_{1}$, o retângulo associado a $v_{1}$ está imediatamente abaixo de $v_{2}$, que está imediatamente abaixo de $v_{3}$ e assim sucessivamente. Por fim, temos que $v_{k}$ está abaixo de $v_{1}$, um absurdo. Logo, não existe um circuito em $\mathbb{G}_{1}$.

Agora, vamos mostrar que existe um único vértice que é fonte.

Como $\mathbb{G}_{1}$ é acíclico, temos que existe ao menos um vértice que não possui aresta entrando. Precisamos mostrar que é único.

Suponha que existe ao menos dois vértices que não possuem aresta entrando. Sejam $u$ e $v$ dois destes vértices.

Pela definição de $\mathbb{G}_{1}$, não existe nenhum retângulo abaixo de $u$ e abaixo de $v$. Isso significa que ambos os vértices incidem na borda inferior da representação retangular de $\mathbb{G}$. Entretanto, temos um absurdo, pois, pela construção de $\mathbb{G}$, só existe um único vértice que incide na borda inferior, o vértice $v_{S}$. Logo, só existe uma única fonte, e esta fonte é o vértice $v_{S}$.

De modo similar, podemos mostrar que só existe um único vértice que é o sorvedouro. Ademais, este deve ser igual a $v_{N}$.

Agora precisamos mostrar que o grafo $\mathbb{G}_{1}$ é conexo. Seja $X$ o conjunto maximal de todos os vértices atingíveis a partir de $v_{S}$.

Suponha que $\mathbb{G}$ não é conexo. Assim, existem ao menos duas componentes conexas. É fácil ver que $X$ é uma das componentes. Agora, perceba que se existir uma componente distinta de $X$, temos uma contradição. Pois, ou existe um outro vértice que não possui aresta entrando, ou existe ao menos um circuito em $\mathbb{G}_{1}$. Logo, $\mathbb{G}_{1}$ é conexo.

Portanto, temos que 1 e 2 são válidos. 
Assim, $\mathbb{G}_{1}$ e $\mathbb{G}_{2}$ podem ser chamados de $s, t$-grafos planos, onde um $s$,t-grafo plano é um grafo plano que só possui uma única fonte $s$ e um único sorvedouro $t$, de modo que $s$ e $t$ pertencem à face externa e existe um caminho de $s$ a todos os outros vértices. Segundo He, as propriedades $s, t$-grafos planares foram estudadas em [KR74, PT88, TV89]. Utilizando estas propriedades, He afirma que a estrutura de $\mathbb{G}_{1}$ pode ser resumida nos seguintes itens:

1. Para cada vértice $v \in V\left(\mathbb{G}_{1}\right) \backslash\left\{v_{S}, v_{N}\right\}$, as arestas entrando em $v$ aparecem consecutivamente ao redor de $v$ em $\mathbb{G}_{1}$. Sejam $e_{1}$ e $e_{2}$, respectivamente, a aresta mais à esquerda e a mais à direita que entram em $v$ em $\mathbb{G}_{1}$. Sejam $e_{3}$ e $e_{4}$, respectivamente, a aresta mais à esquerda e a mais à direita que saem de $v$ em $\mathbb{G}_{1}$. A face de $\mathbb{G}_{1}$ que possui $e_{1}$ e $e_{3}$ em sua borda será denotada por esq $(v)$. Logo, a face de $\mathbb{G}_{1}$ que possui $e_{2}$ e $e_{4}$ será denotada por $\operatorname{dir}(v)$. (Figura 6.5) Para adequarmos a definição de $\operatorname{esq}(\cdot)$ e $\operatorname{dir}(\cdot)$ a todos os vértices de $\mathbb{G}_{1}$, vamos identificar a face externa $\left(f_{\infty}\right)$ de $\mathbb{G}_{1}$ com dois rótulos: $f_{\infty}^{e s q}$ e $f_{\infty}^{d i r}$. Deste modo, considere $e s q\left(v_{O}\right)=f_{\infty}^{e s q}$ e $\operatorname{dir}\left(v_{L}\right)=f_{\infty}^{d i r}$ para os vértices $v_{O}$ e $v_{L}$. Para os vértices $v_{S}$ e $v_{N}$, temos: $\operatorname{esq}\left(v_{S}\right)=e s q\left(v_{N}\right)=f_{\infty}^{e s q} \mathrm{e}$ $\operatorname{dir}\left(v_{S}\right)=\operatorname{dir}\left(v_{N}\right)=f_{\infty}^{d i r}$

2. Para cada face $f$ no interior de $\mathbb{G}_{1}$, temos que a borda de $f$ é definida por dois caminhos disjuntos nas arestas que iniciam no mesmo vértice e terminam em um mesmo vértice. (Figura 6.4A)

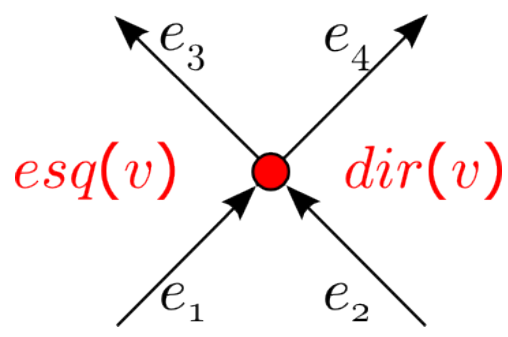

Figura 6.5: Ilustração das definições esq $(\cdot)$ e $\operatorname{dir}(\cdot)$ em $\mathbb{G}_{1}$.

De modo análogo, podemos resumir nos seguintes itens a estrutura de $\mathbb{G}_{2}$ :

1. Para cada vértice $v \in V\left(\mathbb{G}_{2}\right) \backslash\left\{v_{O}, v_{L}\right\}$, as arestas entrando em $v$ aparecem consecutivamente ao redor de $v$ em $\mathbb{G}_{2}$. Sejam $e_{1}$ e $e_{2}$, respectivamente, a aresta mais à esquerda e a mais à direita que entram em $v$ em $\mathbb{G}_{1}$. Sejam $e_{3}$ e $e_{4}$, respectivamente, a aresta mais à esquerda e a mais à direita que saem de $v$ em $\mathbb{G}_{2}$. A face de $\mathbb{G}_{2}$ que possui $e_{1}$ e $e_{3}$ em sua borda será denotada por acima $(v)$. Logo, a face de $\mathbb{G}_{2}$ que possui $e_{2}$ e $e_{4}$ será denotada por abaixo(v). (Figura 6.6) Para adequarmos a definição de $\operatorname{abaixo}(\cdot)$ e $\operatorname{acima}(\cdot)$ a todos os vértices de $\mathbb{G}_{2}$, vamos identificar a face externa $\left(f_{\infty}\right)$ de $\mathbb{G}_{2}$ com dois rótulos: $f_{\infty}^{\text {acima }}$ e $f_{\infty}^{a b a i x o}$. Deste modo, considere $\operatorname{acima}\left(v_{N}\right)=f_{\infty}^{a c i m a}$ e abaixo $\left(v_{S}\right)=f_{\infty}^{\text {abaixo }}$ para os vértices $v_{O}$ e $v_{L}$. Para os vértices $v_{S}$ e $v_{N}$, temos: $\operatorname{acima}\left(v_{O}\right)=\operatorname{acima}\left(v_{L}\right)=f_{\infty}^{\text {acima }}$ e $\operatorname{abaixo}\left(v_{O}\right)=\operatorname{abaixo}\left(v_{L}\right)=f_{\infty}^{\text {abaixo }}$ 
2. Para cada face $f$ no interior de $\mathbb{G}_{2}$, temos que a borda de $f$ é definida por dois caminhos disjuntos nas arestas que iniciam no mesmo vértice e terminam em um mesmo vértice. (Figura 6.4B)

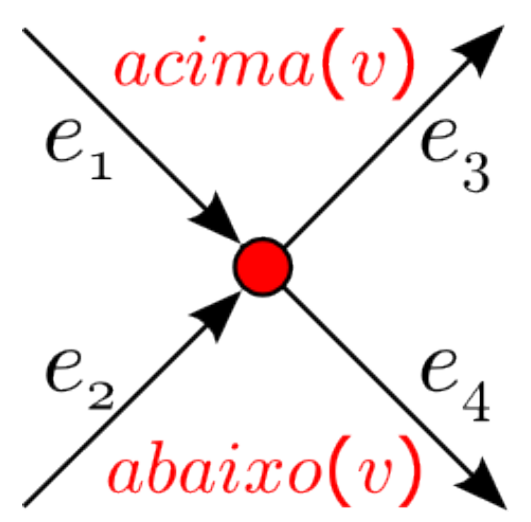

Figura 6.6: $\operatorname{Ilustração~das~definições~acima~}(\cdot)$ e abaixo $(\cdot)$ em $\mathbb{G}_{2}$.

\subsection{Propriedades dAs ROtulaÇÃo REgular DAS ARESTAS}

Considere um grafo $\mathbb{G}$ e $\left\{T_{1}, T_{2}\right\}$ uma rotulação regular das arestas de $\mathbb{G}$. A seguir vamos apresentar definições para uma $S, N$-rede $\mathbb{G}_{1}$ obtida a partir de $\left\{T_{1}, T_{2}\right\}$. De modo análogo, as mesmas definições se aplicam para uma $O, L$-rede $\mathbb{G}_{2}$. Note que $\mathbb{G}_{1}$ e $\mathbb{G}_{2}$ são grafos orientados.

Para cada arco $\alpha \in E\left(\mathbb{G}_{1}\right)$, temos que face $_{e q}(\alpha)$ denota a face que incide a esquerda de $\alpha$ e face $_{\text {dir }}(\alpha)$ denota a face que incide a direita de $\alpha$. De modo similar, para cada arco $\alpha \in E\left(\mathbb{G}_{2}\right)$, temos que face $_{\text {acima }}(\alpha)$ denota a face que incide acima de $\alpha$ e face $_{\text {abaixo }}(\alpha)$ denota a face que incide abaixo de $\alpha$.

Se analisarmos as faces de $\mathbb{G}_{1}\left(\right.$ e $\left.\mathbb{G}_{2}\right)$, existe uma relação de ordem parcial entre elas, se consideramos a relação de qual face está imediatamente à esquerda de outra face. Digamos que uma face $u$ está imediatamente à esquerda (abaixo) de uma face $v$, se existe um arco $\alpha \in E\left(\mathbb{G}_{1}\right)\left(\alpha^{*} \in E\left(\mathbb{G}_{2}\right)\right)$ de modo que face $_{\text {esq }}(\alpha)=u$ e face dir $(\alpha)=v\left(\right.$ face $_{\text {abaixo }}\left(\alpha^{*}\right)=u$ e face $_{\text {acima }}\left(\alpha^{*}\right)=v$ ). Esta relação de ordem induz uma ordenação topológica das faces de $\mathbb{G}_{1}\left(\right.$ e $\left.\mathbb{G}_{2}\right)$, desde a mais à esquerda até a mais à direita (mais abaixo até mais acima).

Pela construção apresentada anteriormente, só existirá uma única face que está à esquerda (abaixo) das outras faces e uma única que está à direita (acima) das outras faces. Assim, se representarmos a relação de ordem parcial de $\mathbb{G}_{1}$ e $\mathbb{G}_{2}$ como grafos, estes grafos serão $s, t$-grafos, pois $\mathbb{G}_{1}$ e $\mathbb{G}_{2}$ são $s, t$-grafos [RT86].

Pela forma que construímos $\mathbb{G}_{1}$, temos que $\mathbb{G}_{1}$ representa as restrições "verticais" do problema de representação retangular, ou seja, as relações de quais retângulos devem estar 
exatamente acima ou abaixo de qualquer retângulo. Ainda, para cada vértice $v$ de $\mathbb{G}_{1}$ definimos $e s q(v)$ e $\operatorname{dir}(v)$ que indicam quais faces incidem à esquerda e à direita de $v$. Perceba que a relação de ordem parcial que foi construída pelas faces de $\mathbb{G}_{1}$ representa as relações entre as arestas verticais da representação retangular de $\mathbb{G}$. Mais precisamente, a relação de quais vértices de $\mathbb{G}_{1}$ devem estar à esquerda ou à direita de uma determinada reta vertical. Basicamente, estamos determinando as coordenadas horizontais (abscissas) das retas verticais da representação retangular a partir das relações de quem está acima ou abaixo.

De modo análogo, a relação de ordem parcial induzida pelas faces de $\mathbb{G}_{2}$ representa as relações entre as arestas horizontais da representação retangular. Assim, as faces do grafo $\mathbb{G}_{2}$ irão determinar as coordenadas verticais (ordenadas) das retas horizontais da representação retangular.

A seguir, vamos mostrar um método de identificar estas retas e criar uma relação de ordem parcial entre elas. Iremos apresentar uma definição genérica e resultados que a relaciona com uma rotulação regular das arestas.

\subsection{NuMERAÇÃO CONSISTENTE DE UM $s, t$-GRAFO}

Definição 6.2 (Numeração consistente de ordem $k$ ): Dado um s,t-grafo $\mathbb{H}$, onde $s$ é a fonte e t é o sorvedouro de $\mathbb{H}$. Uma numeração consistente de ordem $k$ de $\mathbb{H}$ é uma função sobrejetora $\Phi$ que mapeia os vértices de $\mathbb{H}$ no conjunto de inteiros $\{0,1, \ldots, k\}$ de modo que:

(1) $\Phi(s)=0$ e $\Phi(t)=k$;

(2) Para toda aresta $u v \in E(\mathbb{H})$, temos que $\Phi(u)<\Phi(v)$.

Note que os vértices que são fonte e sorvedouro no grafo induzido pela ordenação parcial das faces de $\mathbb{G}_{1}$ são os vértices que representam as faces $f_{\infty}^{e s q}$ e $f_{\infty}^{d i r}$, respectivamente. No grafo induzido pela ordenação parcial das faces de $\mathbb{G}_{2}$ são, respectivamente, os vértices que representam as faces $f_{\infty}^{a b a i x o}$ e $f_{\infty}^{a c i m a}$.

Um exemplo de uma numeração consistente é uma ordenação topológica. Outro exemplo de uma numeração consistente é definir $\Phi(v)$, onde $v \in V(\mathbb{H})$, como sendo igual ao tamanho do maior caminho orientado dá fonte de $\mathbb{H}$ até $v$.

Para implementar um algoritmo que encontra o maior caminho da fonte para qualquer vértice de $\mathbb{H}$, basta modificar o algoritmo de ordenação topológica.

Perceba que qualquer numeração consistente de um $s, t$-grafo $\mathbb{H}$ possui ordem maior ou 
igual ao tamanho do maior caminho da fonte até o sorvedouro de $\mathbb{H}$.

A seguir, vamos mostrar resultados que associam a rotulação regular das arestas $\left\{T_{1}, T_{2}\right\}$ de $\mathbb{G}$ com uma numeração consistente das faces de $\mathbb{G}_{1}$ e $\mathbb{G}_{2}$ que satisfazem a relação de ordem como mostrado.

Sejam $P_{1}$ e $P_{2}$ dois caminhos de $v_{S}$ a $v_{N}$ em $\mathbb{G}_{1}$. Dizemos que $P_{2}$ está a direita de $P_{1}$, se cada aresta de $P_{2}$ pertence a $P_{1}$ ou está desenhado à direita de $P_{1}$. A figura 6.7 ilustra este fato.
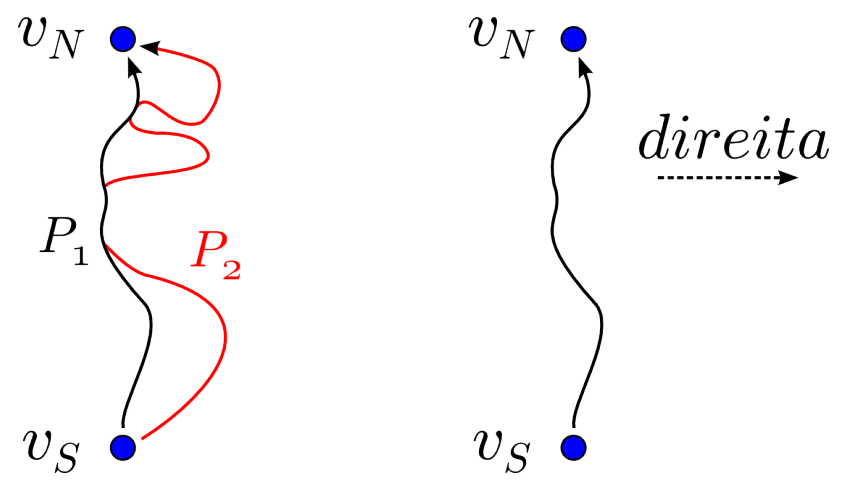

Figura 6.7: Ilustração de um caminho $P_{2}$ a direita do caminho $P_{1}$.

Conforme já mencionado anteriormente, cada face interna de $\mathbb{G}_{1}$ é composta por dois caminhos orientados de modo que cada face possui um único vértice que é fonte e um único vértice que é sorvedouro. Seja $f$ uma face de $\mathbb{G}_{1}$, vamos chamar os caminhos que formam a face $f$ de: caminho esquerdo e de caminho direito da borda de $f$. A figura 6.8 ilustra estes caminhos. Note que como dividimos a face externa em duas partes, definimos que o caminho esquerdo de $f_{\infty}^{e s q}$ e o caminho direito de $f_{\infty}^{\text {dir }}$ não existem. Entretanto, o caminho direito de $f_{\infty}^{e s q}$ é $\left\langle v_{S} v_{O}, v_{O} v_{N}\right\rangle$, enquanto o caminho esquerdo de $f_{\infty}^{\text {dir }}$ é $\left\langle v_{S} v_{L}, v_{L} v_{N}\right\rangle$.

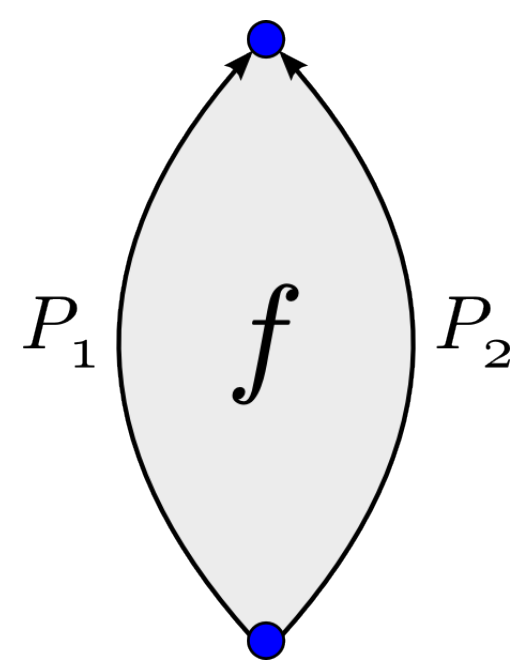

Figura 6.8: Ilustração de uma face $f$ e os caminhos que a formam. O caminho $P_{1}$ é o caminho esquerdo da borda de $f$, enquanto o caminho $P_{2}$ é o caminho direito da borda de $f$.

Um sistema de caminhos de $\mathbb{G}_{1}$ é uma coleção de caminhos de $v_{S}$ a $v_{N}$ em $\mathbb{G}_{1}$ 
$\left\{P_{0}, P_{1}, \ldots, P_{l-1}\right\}$, onde:

(1) $\bigcup_{i=0}^{l-1} E\left(P_{i}\right)=E\left(\mathbb{G}_{1}\right)$;

(2) $\quad P_{i}$ está a direita de $P_{i-1}$, para $1 \leq i \leq l-1$.

Agora, considere uma numeração consistente $\Phi$ das faces de $\mathbb{G}_{1}$ de ordem $k$. Define-se para cada $i$ tal que $0 \leq i \leq k$ :

$F_{i}\left(\mathbb{G}_{1}\right)=\left\{f: f \in F\left(\mathbb{G}_{1}\right)\right.$ e $\left.\Phi(f)=i\right\}$

$E_{\text {esq }}^{i}\left(\mathbb{G}_{1}\right)=\left\{\alpha: \alpha \in E\left(\mathbb{G}_{1}\right)\right.$ e $\alpha$ pertence a um caminho esquerdo de uma face $f$ tal que $\left.f \in F_{i}\left(\mathbb{G}_{1}\right)\right\}$,

$E_{d i r}^{i}\left(\mathbb{G}_{1}\right)=\left\{\alpha: \alpha \in E\left(\mathbb{G}_{1}\right)\right.$ e $\alpha$ pertence a um caminho direito de uma face $f$ tal que $\left.f \in F_{i}\left(\mathbb{G}_{1}\right)\right\}$.

Considere a seguinte coleção de caminhos $\mathfrak{S}=\left\{P_{0}, \ldots, P_{k-1}\right\}$ de $v_{S}$ a $v_{N}$ em $\mathbb{G}_{1}$, onde:

- $P_{0}=E_{d i r}^{0}\left(\mathbb{G}_{1}\right)$,

- $P_{i}=\left(P_{i-1} \backslash E_{e s q}^{i}\left(\mathbb{G}_{1}\right)\right) \cup E_{d i r}^{i}\left(\mathbb{G}_{1}\right)$, para $1 \leq i \leq k-1$.

Perceba que:

$$
\begin{array}{ll}
F_{0}\left(\mathbb{G}_{1}\right)=\left\{f_{\infty}^{e s q}\right\}, & E_{\text {esq }}^{0}\left(\mathbb{G}_{1}\right)=\emptyset, \\
& E_{d i r}^{0}\left(\mathbb{G}_{1}\right)=\left\{v_{S} v_{O}, v_{O} v_{N}\right\} . \\
F_{k}\left(\mathbb{G}_{1}\right)=\left\{f_{\infty}^{d i r}\right\}, & E_{\text {esq }}^{k}\left(\mathbb{G}_{1}\right)=\left\{v_{S} v_{L}, v_{L} v_{N}\right\}, \\
& E_{d i r}^{0}\left(\mathbb{G}_{1}\right)=\emptyset .
\end{array}
$$

A figura 6.9 ilustra um exemplo de um sistema de caminhos $\mathfrak{S}=\left\{P_{0}, P_{1}, P_{2}, P_{3}, P_{4}, P_{5}\right\}$, onde $E\left(P_{0}\right)=\left\{v_{S} v_{O}, v_{0} v_{N}\right\}, E\left(P_{1}\right)=\{a, b, c, d, e, f\}, E\left(P_{2}\right)=\{g, h, c, i, f\}$, $E\left(P_{3}\right)=\{j, k, l, r, i, f\}, E\left(P_{4}\right)=\{m, n, o, r, i, f\}$ e $E\left(P_{5}\right)=\left\{v_{S} v_{L}, v_{L} v_{N}\right\}$.

Precisamos provar que $\mathfrak{S}$ é um sistema de caminhos de $\mathbb{G}_{1}$. Entretanto, antes precisamos fazer algumas observações relativas às definições que acabamos de introduzir.

Queremos mostrar que o conjunto das arestas de $\mathbb{G}_{1}$ pode ser particionado nos conjuntos $E_{\text {dir }}^{i}\left(\mathbb{G}_{1}\right)$ para $0 \leq i<k$.

Seja $\alpha \in E\left(\mathbb{G}_{1}\right), p=\Phi\left(\right.$ face $\left._{\text {esq }}(\alpha)\right)$ e $q=\Phi\left(\right.$ face $\left._{\text {dir }}(\alpha)\right)$. Como $\alpha$ pertence ao caminho esquerdo de face $_{\text {dir }}(\alpha)$ e ao caminho direito de face $_{\text {esq }}(\alpha)$, temos que $\alpha \in E_{d i r}^{p}\left(\mathbb{G}_{1}\right)$ e $\alpha \in$ 


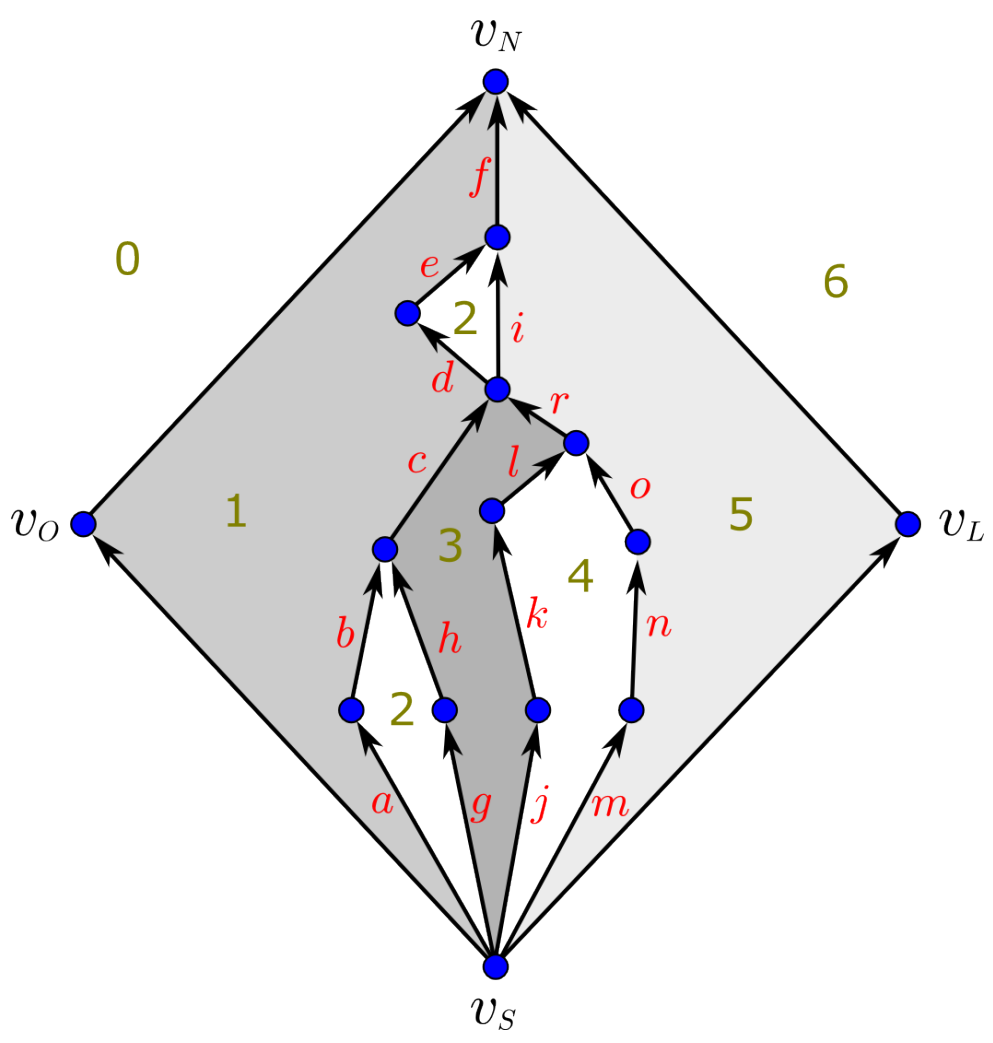

Figura 6.9: Ilustração de um sistema de caminhos $\mathfrak{S}$.

$E_{\text {esq }}^{q}\left(\mathbb{G}_{1}\right)$. Logo, $p<q$, uma vez que a relação de ordem das faces de $\mathbb{G}_{1}$ implica que a face face $_{\text {esq }}(\alpha)$ deve ser menor que face $_{\text {dir }}(\alpha)$.

A aresta $\alpha^{*}$ correspondente a $\alpha$ em $\mathbb{G}_{1}^{*}$ é orientada de $\operatorname{face}_{e s q}(\alpha)$ para $f a c e_{\text {dir }}(\alpha), \operatorname{logo}$, pela definição de $\Phi, p<q$.

Deste modo, temos que $E_{\text {esq }}^{i}\left(\mathbb{G}_{1}\right) \cap E_{\text {dir }}^{i}\left(\mathbb{G}_{1}\right)=\emptyset$ para $0 \leq i \leq k$. Ademais, como cada $\alpha \in E\left(\mathbb{G}_{1}\right)$ pertence a exatamente um conjunto $E_{\text {dir }}^{i}\left(\mathbb{G}_{1}\right)$, para $0 \leq i<k$ temos que $E\left(\mathbb{G}_{1}\right)$ é a união disjunta de $E_{\text {dir }}^{i}\left(\mathbb{G}_{1}\right)$ para $0 \leq i<k$.

De modo análogo, podemos mostrar que $E\left(\mathbb{G}_{1}\right)$ é a união disjunta de $E_{\text {esq }}^{i}\left(\mathbb{G}_{1}\right)$ para $0<i \leq k$.

Lema 6.2: Seja $\Phi$ uma numeração consistente das faces de $\mathbb{G}_{1}$. A coleção $\mathfrak{S}=$ $\left\{P_{0}, \ldots, P_{k-1}\right\}$ é um sistema de caminhos de $\mathbb{G}_{1}$.

Demonstração: Vamos provar por indução em $i(0 \leq i<k)$ que:

(1) $P_{i}$ é um caminho de $v_{S}$ a $v_{N}$ em $\mathbb{G}_{1}$;

(2) $E_{e s q}^{i+1} \subseteq P_{i}$.

O caso base é quando $i=0$.

Assim, $P_{0}=\left\langle v_{S}, v_{O}, v_{N}\right\rangle$ é um caminho de $v_{S}$ a $v_{N}$ em $\mathbb{G}_{1}$. Logo, a propriedade (1) segue. 
Para a propriedade $(2)$, seja $\alpha \in E_{\text {esq }}^{1}\left(\mathbb{G}_{1}\right)$. Então, $\alpha$ pertence ao caminho esquerdo de $f$, onde $f \in F_{1}\left(\mathbb{G}_{1}\right)$. Ademais, $f=$ face $_{\text {dir }}(() \alpha)$. Seja $g=$ face $_{\text {esq }}(() \alpha)$. Como $\Phi(f)=1$, pela ordem parcial das faces de $\mathbb{G}_{1}$, temos que $\operatorname{Phi}(g)<1$, logo $\operatorname{Phi}(g)=0$, implicando que $g=f_{\infty}^{e s q}$. Portanto, $\alpha \in E_{\text {dir }}^{0}\left(\mathbb{G}_{1}\right)$ e $E_{\text {dir }}^{0}\left(\mathbb{G}_{1}\right) \subseteq P_{0}$.

Considere que as propriedades (1) e (2) são válidas para $i-1<k-1$.

Agora, devemos mostrar que (1) e (2) valem para $i$.

Para mostrar a propriedade (1), temos pela hipótese indutiva que $P_{i}$ é um caminho de $v_{S}$ a $v_{N}$ em $\mathbb{G}_{1}$. Considere $F_{i}\left(\mathbb{G}_{1}\right)=\left\{f_{1}, \ldots, f_{l}\right\}$. Seja $A_{j}$ e $B_{j}$ o caminho da esquerda e o caminho da direita, respectivamente, de $f_{j}$, para $1 \leq j \leq l$. Como $E_{\text {esq }}^{i} \subseteq P_{i-1}$, os caminhos $A_{j}$, para $1 \leq j \leq l$ são segmentos disjuntos nas arestas de $P_{i-1}$. Como $A_{j}$ e $B_{j}$ iniciam e terminam nos mesmos vértices. Logo, obtemos $P_{i}$ trocando em $P_{i-1}$ cada segmento de $A_{j}$ por $B_{j}$. Assim, $P_{i}$ é um caminho de $v_{S}$ a $v_{N}$ em $\mathbb{G}_{1}$. Logo, a propriedade (1) é válida para $i$.

Agora, vamos provar que a propriedade (2) também é válida para $i$.

Seja $\alpha$ uma aresta qualquer de $E_{\text {esq }}^{i+1}\left(\mathbb{G}_{1}\right)$. Seja $f_{1}=$ face $_{\text {esq }}(\alpha)$ e $f_{2}=$ face $_{\text {dir }}(\alpha)$.

Como $\alpha \in E_{\text {esq }}^{i+1}\left(\mathbb{G}_{1}\right)$, então $\Phi\left(f_{2}\right)=i+1$. Suponha que $\Phi\left(f_{1}\right)=q$ para algum inteiro $q$. Então, $\alpha \in E_{\text {dir }}^{q}\left(\mathbb{G}_{1}\right)$. Assim, pela escolha de $\alpha, f_{1}$ está imediatamente à esquerda de $f_{2}$. Logo, pela ordem parcial das faces de $\mathbb{G}_{1}$ e da numeração consistente $\Phi$, temos que $q<i+1$.

Pela definição de $\mathfrak{S}, \alpha$ foi adicionada na construção do caminho $P_{q}$ e deletado quando o caminho $P_{i+1}$ foi construído. Então $\alpha \in P_{j}$, para todo $q \leq q \leq i$. Logo, como $\alpha \in P_{i}$, concluímos que $E_{e s q}^{i+1}\left(\mathbb{G}_{1}\right) \subseteq P_{i}$. Completando assim a indução.

Com a propriedade (1) e (2) podemos provar o lema. Para cada aresta $\alpha$ de $E\left(\mathbb{G}_{1}\right)$, temos que $\alpha$ pertence a algum conjunto $E_{d i r}^{i}\left(\mathbb{G}_{1}\right)$, para $0 \leq i \leq k-1$ e assim $\alpha \in P_{i}$. Deste modo, $\bigcup_{i=0}^{k-1} E\left(P_{i}\right)=E\left(\mathbb{G}_{1}\right)$. Ainda, pela forma que os caminhos $P_{i}$ são construídos, é fácil ver que $P_{i}$ está a direita de $P_{i-1}$ para todo $1 \leq i \leq k-1$. Portanto, $\mathfrak{S}$ é um sistema de caminhos de $\mathbb{G}_{1}$.

Lema 6.3: Seja $\Phi$ uma numeração consistente de $\mathbb{G}_{1}^{*}$. Para cada vértice $v \in V\left(\mathbb{G}_{1}\right)$, $f_{1}=e s q(v)$ e $f_{2}=\operatorname{dir}(v)$. Defina $x_{1}(v)=\Phi\left(f_{1}\right)$ e $x_{2}=\Phi\left(f_{2}\right)$. Então, $v \in V\left(P_{i}\right)$ se e somente se $x_{1}(v) \leq i<x_{2}(v)$.

Demonstração: Como $v$ pertence ao caminho direito de $f_{1}$, temos que $v \in V\left(P_{x_{1}(v)}\right)$. Entretanto, como $v$ também pertence ao caminho esquerdo de $f_{2}$, temos que $v \notin V\left(P_{x_{2}(v)}\right)$. Ademais, $v$ foi inserido pela primeira vez no caminho de $v_{S}$ a $v_{N}$ justamente no caminho $P_{x_{1}(v)}$ e permaneceu em nos caminhos de $v_{S}$ a $v_{N}$ até ser removido no caminho $P_{x_{2}(v)}$. Portanto, temos que $v$ pertence a todo caminho $P_{i}$, onde $x_{1}(v) \leq i<x_{2}(v)$.

Até o momento, mostramos somente resultados para $S, N$-rede $\mathbb{G}_{1}$. Entretanto, os mesmos 
resultados se aplicam para $O, L$-rede $\mathbb{G}_{2}$, com as alterações necessárias.

Lema 6.4: Sejam $\mathbb{G}_{1}$ e $\mathbb{G}_{2}$, respectivamente, as $S, N$-rede e $O, L$-rede de $\mathbb{G}$ a partir de uma rotulação regular das arestas $\left\{T_{1}, T_{2}\right\}$. Seja $\Phi_{1}$ uma numeração consistente das faces de $\mathbb{G}_{1}$ e $\Phi_{2}$ uma numeração consistente das faces de $\mathbb{G}_{2}$. Considere $u, v \in V(\mathbb{G})$, então as seguintes proposições são válidas:

(1) Se a aresta $u v \in T_{2}$, então $x_{2}(u)=x_{1}(v)$.

(2) Se existe um caminho de $u$ a $v$ em $\mathbb{G}_{2}$ de tamanho maior ou igual a 2 , então $x_{2}(u)<x_{1}(v)$.

(3) Se a aresta $u v \in T_{1}$, então $y_{2}(u)=y_{1}(v)$.

(4) Se existe um caminho de $u$ a $v$ em $\mathbb{G}_{1}$ de tamanho maior ou igual a 2, então $y_{2}(u)<y_{1}(v)$.

Demonstração: Iremos apresentar a prova somente dos itens (1) e (2), pois a prova de (3) e (4) é similar.

Inicialmente, vamos provar (1). Suponha que $u v \in T_{2}$.

Para qualquer vértice $w \in V\left(\mathbb{G}_{2}\right)$, se olharmos as arestas, por exemplo, no sentido horário temos um conjunto de arestas saindo de $w$ e um conjunto saindo de $w$. (Figura 6.10)

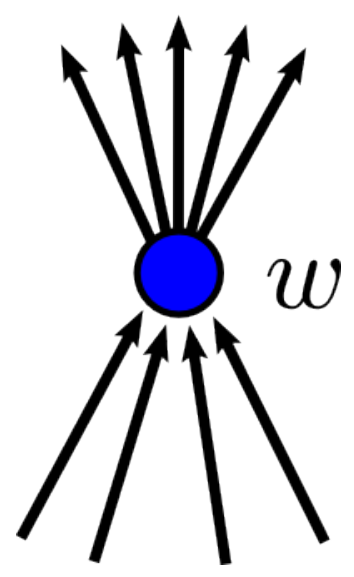

Figura 6.10: Representação das arestas que incidem em um vértice $w$ em $\mathbb{G}_{1}$.

Vamos rotular algumas arestas de $u$ e de $v$ em $\mathbb{G}_{1}$. Seja $e_{1}$ a aresta mais à direita que está saindo de $u$ e $e_{2}$ a aresta mais à direita que está entrando em $u$. Seja $e_{3}$ a aresta mais à esquerda que está saindo de $v$ e $e_{4}$ a aresta mais à esquerda que está entrando em $v$. A figura 6.11 ilustra as arestas $e_{1}, e_{2}, e_{3}$ e $e_{4}$ em $\mathbb{G}_{1}$.

Seja $f$ a face em $\mathbb{G}_{1}$ que contém em sua borda as arestas $e_{1}, e_{2}, e_{3}$ e $e_{4}$.

Então, $f=\operatorname{dir}(u)=e s q(v)$. Implicando que $x_{2}(u)=x_{1}(v)=\Phi(f)$. Logo, a propriedade (1) é válida. 


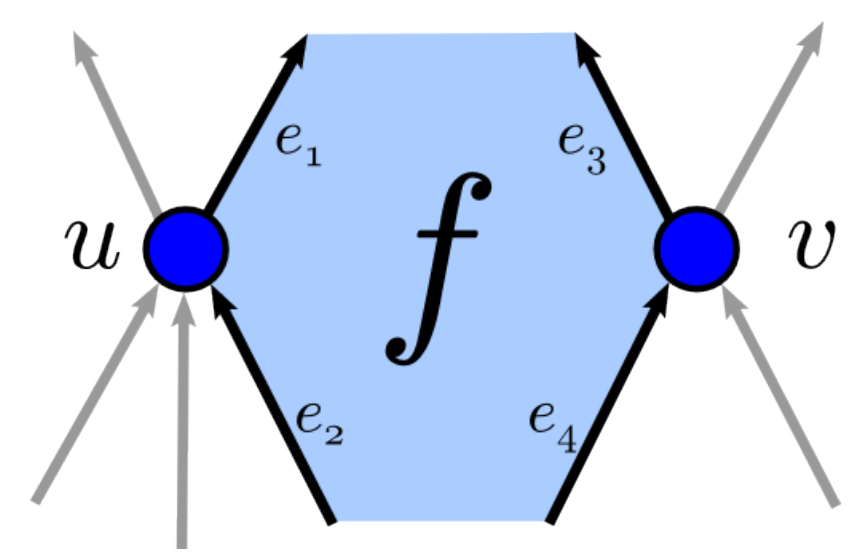

Figura 6.11: Arestas $e_{1}, e_{2}, e_{3}$ e e $e_{4}$ que incidem no vértice $u$ e $v$ em $\mathbb{G}_{1}$.

Agora, vamos provar (2).

Seja $\left\langle u=u_{0}, u_{1}, u_{2}, \ldots, u_{p}=v\right\rangle$, onde $p \geq 2$, um caminho em $\mathbb{G}_{2}$. Por $(1), x_{2}\left(u_{j-1}\right)=$ $x_{1}\left(u_{j}\right)$, para todo $j$ tal que $1 \leq j \leq p$. Como $x_{1}\left(u_{j}\right)<x_{2}\left(u_{j}\right)$ para todo $j$ tal que $0 \leq j \leq p$ e $p \geq 2$, temos que $x_{2}(u)=x_{2}\left(u_{0}\right)<x_{1}\left(u_{p}\right)=x_{1}(v)$.

\subsection{Algoritmo De He BASEAdo EM $s, t$-CAMinhos}

Baseado nos resultados apresentados na seção anterior, apresentamos o algoritmo Algoritmo-He.

Seja $\mathcal{F}$ um grafo plano triangulado. Considere $\mathbb{G}=\mathbb{H}-v_{\infty}$, onde $\mathbb{H}$ é a 4 -extensão de $\mathcal{F}$. O algoritmo Algoritmo-He recebe como entrada um grafo $\mathbb{G}$ e devolve uma representação retangular de $\mathcal{F}$. Existem duas funções em Algoritmo-He, DefinirX e DEFINIRY, que calculam, respectivamente, as abscissas das retas verticais e as ordenadas das retas horizontais de cada retângulo da representação retangular de $\mathbb{G}$.

Para cada vértice $v \in V(\mathbb{G})$ temos quatro variáveis: $x_{1}(v), x_{2}(v), y_{1}(v)$ e $y_{2}(v)$. Onde $x_{1}(v)$ e $x_{2}(v)$ são as abscissas das retas verticais do retângulo que representa $v$ na representação retangular. De modo análogo, $y_{1}(v)$ e $y_{2}(v)$ são as ordenadas das retas horizontais do retângulo que representa $v$. 
Algoritmo Algoritmo-He $(\mathbb{G})$

ENTRADA: Grafo $\mathbb{G}$.

SAÍDA: Representação retangular de um grafo plano triangulado utilizando $\mathbb{G}$.

1. Encontrar uma rotulação regular das arestas $\left\{T_{1}, T_{2}\right\}$ de $\mathbb{G}$;

2. DEFINIRX $\left(T_{1}, T_{2}\right)$;

3. DefinirY $\left(T_{1}, T_{2}\right)$;

4. Para cada $v \in V(\mathbb{G})$ faça

5. Defina o retângulo que representa $v$ na representação retangular de $\mathbb{G}$, como sendo composto por dois segmentos de retas verticais com abscissas $x_{1}(v)$ e $x_{2}(v) \mathrm{e}$ dois segmentos de retas horizontais com ordenadas $y_{1}(v)$ e $y_{2}(v)$;

Algoritmo DefiniRX $\left(T_{1}, T_{2}\right)$

ENTRADA: Rotulação regular das arestas $\left\{T_{1}, T_{2}\right\}$.

SAÍDA: Abscissas das retas verticais de todos os retângulos da representação retangular.

1. Construa uma $S, N$-rede $\mathbb{G}_{1}$ a partir de $\left\{T_{1}, T_{2}\right\}$;

2. Encontre uma numeração consistente $\Phi$ das faces de $\mathbb{G}_{1}$;

3. Seja $k=\Phi\left(f_{\infty}^{\text {dir }}\right)$;

4. Para cada $v \in V(\mathbb{G}) \backslash\left\{v_{S}, v_{N}\right\}$ faça

5. $\quad$ Seja $f_{1}=e s q(v)$ e $f_{2}=\operatorname{dir}(v)$;

6. $\quad x_{1}(v)=\Phi\left(f_{1}\right)$ e $x_{2}(v)=\Phi\left(f_{2}\right)$;

7. $\quad x_{1}\left(v_{N}\right)=x_{1}\left(v_{S}\right)=1$ e $x_{2}\left(v_{N}\right)=x_{2}\left(v_{S}\right)=k-1$;

Algoritmo DeFiniRY $\left(T_{1}, T_{2}\right)$

ENTRADA: Rotulação regular das arestas $\left\{T_{1}, T_{2}\right\}$.

SAÍDA: Ordenadas das retas horizontais de todos os retângulos da representação retangular.

1. Construa uma $O, L$-rede $\mathbb{G}_{2}$ a partir de $\left\{T_{1}, T_{2}\right\}$;

2. Encontre uma numeração consistente $\Phi$ das faces de $\mathbb{G}_{2}^{*}$;

3. Seja $k=\Phi\left(f_{\infty}^{a c i m a}\right)$;

4. Para cada $v \in V(\mathbb{G})$ faça

5. Seja $f_{1}=\operatorname{abaixo}(v)$ e $f_{2}=\operatorname{acima}(v)$;

6. $\quad y_{1}(v)=\Phi\left(f_{1}\right)$ e $y_{2}(v)=\Phi\left(f_{2}\right)$;

Teorema 6.5: O algoritmo Algoritmo-He possui complexidade de tempo linear.

Demonstração: Para mostrar que o Algoritmo-He possui tempo linear precisamos mostrar que os algoritmos DefiniRX e DefiniRY possuem complexidade linear. Vamos 
apresentar uma análise para o algoritmo DEFiniRY. Para o algoritmo DEFInIRX a análise é similar.

A primeira operação do algoritmo DefiniRY consiste em construir o grafo induzido pelas arestas $T_{2}$. Isso pode ser feito em tempo linear. Encontrar uma numeração consistente depende diretamente da função $\Phi$. Entretanto, podemos implementá-la utilizando o algoritmo de ordenação topológica. Cada operação das linhas 4-6 possuem complexidade constante, entretanto elas serão executadas um número linear de vezes, logo, a execução de todas operações das linhas 4-6 consomem tempo linear. Assim, o algoritmo DefiniRY possui complexidade linear.

A operação da linha 1 do Algoritmo-He pode ser executada em tempo linear utilizando o algoritmo Construir-GCD apresentado no capítulo 5. O algoritmo Construir-GCD constrói um grafo de caminhos dirigidos, que pode ser utilizado para definir $T_{1}$ e $T_{2}$. As linhas 2 e 3 possuem complexidade linear. Por fim, as linhas 4-5 são operações constantes que são executadas um número linear de vezes. Portanto, todas as operações possuem complexidade linear, logo, o Algoritmo-He possui complexidade linear.

Teorema 6.6: $\quad O$ algoritmo Algoritmo-He encontra uma representação retangular do grafo de entrada $\mathbb{G}$.

Demonstração: Para provar a corretude do algoritmo, considere uma rotulação regular das arestas $\left\{T_{1}, T_{2}\right\}$. A partir de $\left\{T_{1}, T_{2}\right\}$, construa a $S, N$-rede $\mathbb{G}_{1}$ e a $O, L$-rede $\mathbb{G}_{2}$ de $\mathbb{G}$. Seja $\Phi_{1}$ uma numeração consistente de $\mathbb{G}_{1}$ e $\Phi_{2}$ para $\mathbb{G}_{2}$.

Seja $\mathfrak{S}_{1}=\left\{P_{0}, \ldots, P_{k_{1}-1}\right\}$ um sistema de caminhos de $\mathbb{G}_{1}$ derivado de $\Phi_{1}$, onde:

- $P_{0}=E_{d i r}^{0}\left(\mathbb{G}_{1}\right)$,

- $P_{i}=\left(P_{i-1} \backslash E_{\text {esq }}^{i}\left(\mathbb{G}_{1}\right)\right) \cup E_{\text {dir }}^{i}\left(\mathbb{G}_{1}\right)$, para $1 \leq i \leq k_{1}-1$.

Seja $\mathfrak{S}_{2}=\left\{Q_{0}, \ldots, Q_{k_{2}-1}\right\}$ um sistema de caminhos de $\mathbb{G}_{2}$ derivado de $\Phi_{2}$, onde:

- $P_{0}=E_{\text {acima }}^{0}\left(\mathbb{G}_{2}\right)$,

- $P_{i}=\left(P_{i-1} \backslash E_{\text {abaixo }}^{i}\left(\mathbb{G}_{2}\right)\right) \cup E_{\text {acima }}^{i}\left(\mathbb{G}_{2}\right)$, para $1 \leq i \leq k_{2}-1$.

Mostramos no lema 6.2 que $\mathfrak{S}_{1}$ é um sistema de caminhos de $\mathbb{G}_{1}$. De modo análogo, temos que $\mathfrak{S}_{2}$ é um sistema de caminhos de $\mathbb{G}_{2}$.

Para cada $v \in V(\mathbb{G})$, denotamos por $R(v)$ o retângulo na representação retangular correspondente ao vértice $v$.

No grafo da representação retangular construído pelo Algoritmo-HE, cada caminho $P_{i} \in \mathfrak{S}_{1}, 0 \leq i \leq k_{1}-1$, corresponde a uma faixa vertical limitada por duas retas verticais com abcissas $i$ e $i+1$. Podemos pensar que estamos removendo as linhas horizontais da 
representação e o caminho $P_{i}$ é a região limitada por duas retas. De modo similar, cada caminho $Q_{j} \in \mathfrak{S}_{2}, 0 \leq j \leq k_{2}-1$, corresponde a uma faixa horizontal limitada por duas retas horizontais de ordenadas $j$ e $j+1$.

As coordenadas do retângulo $R(v), v \in V(\mathbb{G})$ da representação retangular é dada por $x_{1}(v), x_{2}(v), y_{1}(v)$ e $y_{2}(v)$. Para mostrar que o conjunto $\{R(v): v \in V(\mathbb{G})\}$ forma uma representação retangular de $\mathbb{G}$. Assim, precisamos mostrar: (1) A representação retangular de $\mathbb{G}$ não possui buracos; e (2) Existe uma aresta $u v \in E(\mathbb{G})$ se e somente se $R(u)$ e $R(v)$ são adjacentes.

Primeiramente, vamos mostrar (1). Como a representação retangular de $\mathbb{G}$ possui largura $k_{1}-1$ e altura $k_{2}-1$ e todos os vértices possuem coordenadas inteiras, para provar que não existe buracos, precisamos mostrar que para todo quadrado limitado pelas coordenadas cartesianas $(i, j)$ e $(i+1, j+1)\left(0 \leq i \leq k_{1}-1\right.$ e $\left.0 \leq j \leq k_{2}-1\right)$, existe um único retângulo $R(v)$ tal que $v \in V(\mathbb{G})$. Considere $P_{i} \in \mathfrak{S}_{1}$ e $Q_{i} \in \mathfrak{S}_{2}$. Exceto em quatro casos: (a) $i=0, j=0$, (b) $i=k_{1}-1, j=0$, (c) $i=0, j=k_{2}-1$ e (d) $i=k_{1}-1, j=k_{2}-1, P_{i}$ e $P_{j}$ intersectam-se em um único vértice $v \in V(\mathbb{G})$. Vamos mostrar para o caso geral, depois trataremos para estes casos especiais.

Pelo lema 6.3, $v$ é o único vértice que satisfaz as seguintes inequações: $x_{1}(v) \leq i ; i+1 \leq$ $x_{2}(v) ; y_{1}(v) \leq j$; e $j+1 \leq y_{2}(v)$. Logo, $R(v)$, é o único retângulo que o quadrado definido pelas coordenadas $(i, j)$ e $(i+1, j+1)$ pertence.

Para os quatro casos especiais a afirmação (1) não é verdade, por exemplo, $v_{S}$ e $v_{O}$ pertencem a interseção de $P_{0}$ e $Q_{0}$. Estes casos correspondem aos quatro quadrados unitários que estão nos cantos da representação retangular. Entretanto, na linha 8 do algoritmo DEFiniRX, garantimos que estes quadrados irão pertencer somente a $R\left(v_{O}\right)$ ou a $R\left(v_{L}\right)$. Logo, não existe buracos na representação retangular.

Para provar a propriedade (2), vamos dividí-la em duas partes. Inicialmente, iremos mostrar que se existe uma aresta $u v$ em $\mathbb{G}$, então $R(u)$ e $R(v)$ devem compartilhar algum segmento de seus lados. Depois, iremos mostrar que se $R(u)$ e $R(v)$ compartilham algum segmento de seus lados, então existe a aresta $u v$ em $\mathbb{G}$.

Seja $\alpha=u v$ uma aresta de $\mathbb{G}$. Se $\alpha$ incide na face externa de $\mathbb{G}$,então, pela construção de $\mathbb{G}$, é fácil ver que os retângulos $R\left(v_{S}\right), R\left(v_{L}\right), R\left(v_{N}\right)$ e $R\left(v_{O}\right)$ satisfazem as restrições de todas arestas incidentes na face externa, a saber: $v_{S} v_{O}, v_{O} v_{N}, v_{N} v_{L}$ e $v_{L} v_{S}$.

Assim, podemos considerar que $\alpha$ é uma aresta que não incide na face externa de $\mathbb{G}$. Suponha que $\alpha \in T_{1}$ e é orientada de $u$ para $v$ (os outros casos são similares). Seja $P_{i} \in \mathfrak{S}_{1}$, para algum $i$ de modo que $\alpha \in V\left(P_{i}\right)$. Pelo lema 6.3, temos que $x_{1}(u) \leq i \leq x_{2}(u)-1$ e $x_{1}(v) \leq i \leq x_{2}(v)$. Pelo lema 6.4(3), $y_{2}(u)=y_{1}(v)=j$, para algum $j$. Logo, $R(u)$ e $R(v)$ compartilham o segmento de reta de seus lados definido pelos pontos $(i, j)$ e $(i+1, j)$.

Agora, vamos provar que se $R(u)$ e $R(v)$ compartilham algum segmento de seus lados, 
então, a aresta $u v$ pertence a $\mathbb{G}$.

Suponha que segmento que é compartilhado por $R(u)$ e $R(v)$ é um segmento horizontal $L$ definido pelos pontos $(i, j)$ e $(i+1, j)$. Igualmente ao apresentado anteriormente, os outros casos são similares.

Como $x_{1}(u) \leq i ; i+1 \leq x_{2}(u) ; x_{1}(v) \leq i$ e $i+1 \leq x_{2}(v)$, temos que $u$ e $v$ pertencem ao caminho $P_{i}$ de $\mathfrak{S}_{1}$. Agora, precisamos mostrar que $u v$ é uma aresta em $P_{i}$. Suponha por absurdo que existe um caminho direcionado de $u$ a $v$ em $\mathbb{G}_{1}$ com pelo menos duas arestas. Pelo lema 6.4 temos que $y_{2}(u)<y_{1}(v)$, logo, uma contradição com o fato de $L$ ser um segmento de reta que pertence a $R(u)$ e $R(v)$.

Logo, como (1) e (2) são válidos, temos que $\{R(v): v \in V(\mathbb{G})\}$ é uma representação retangular de $\mathbb{G}$. 



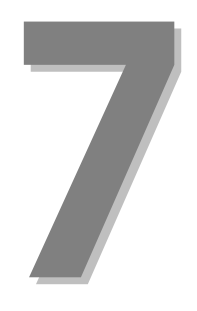

REDUÇÃO DO PROBLEMA DE REPRESENTAÇÃO RETANGULAR AO PROBLEMA DE ENCONTRAR UM EMPARELHAMENTO PERFEITO EM GRAFOS BIPARTIDOS

Lai e Leinwand [LL90] apresentam uma redução dos problema de decisão e construção da representação retangular ao problema de encontrar um emparelhamento perfeito em um grafo bipartido.

Neste capítulo, considere que o grafo de entrada $\mathbb{T}$ é um grafo plano triangulado. Um grafo plano triangulado é um grafo plano em que suas faces, com exceção da face externa, são triangulares. Porém, como as figuras 7.1 e 7.2 ilustram, o algoritmo de Lai e Leinwand necessita, para que o grafo $\mathbb{T}$ admita uma representação retangular $\mathbb{R}$, que estendemos $\mathbb{T}$ de modo que exista um vértice que represente a face externa da representação retangular $\mathbb{R}$ de $\mathbb{T}$ e que algumas faces representem os 4 cantos do retângulo mais externo de $\mathbb{R}$.

Para isso, vamos construir um grafo $\mathbb{G}$ que será uma extensão de $\mathbb{T}$. Inicialmente, $\mathbb{G}$ é uma cópia de $\mathbb{T}$. Vamos adicionar um vértice $v_{\infty}$ a $\mathbb{G}$ de modo que $v_{\infty}$ represente a face 


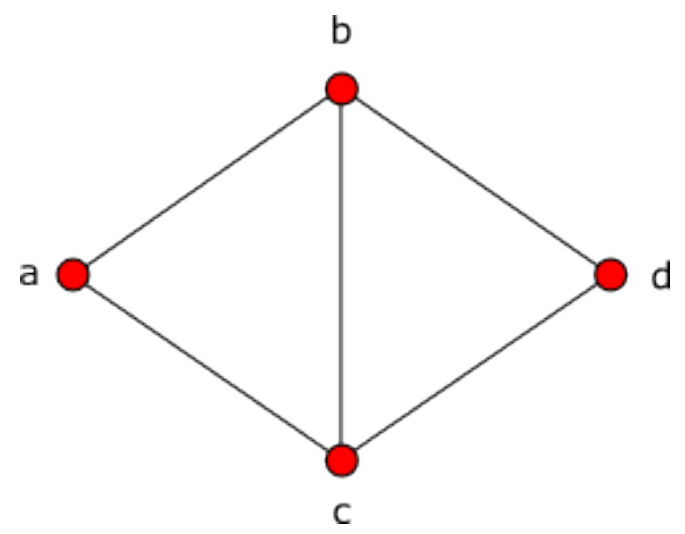

(A)

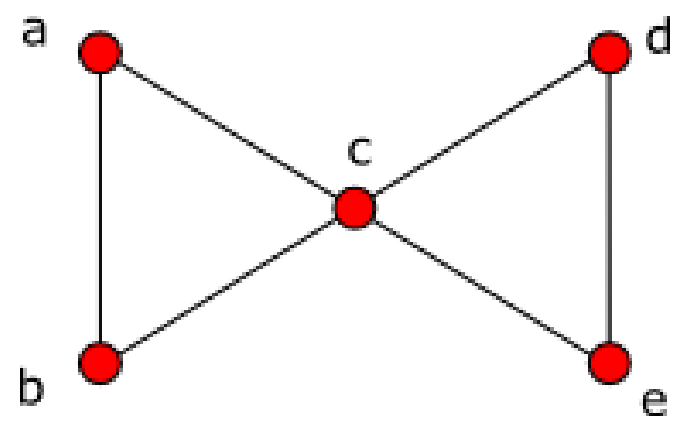

(C)

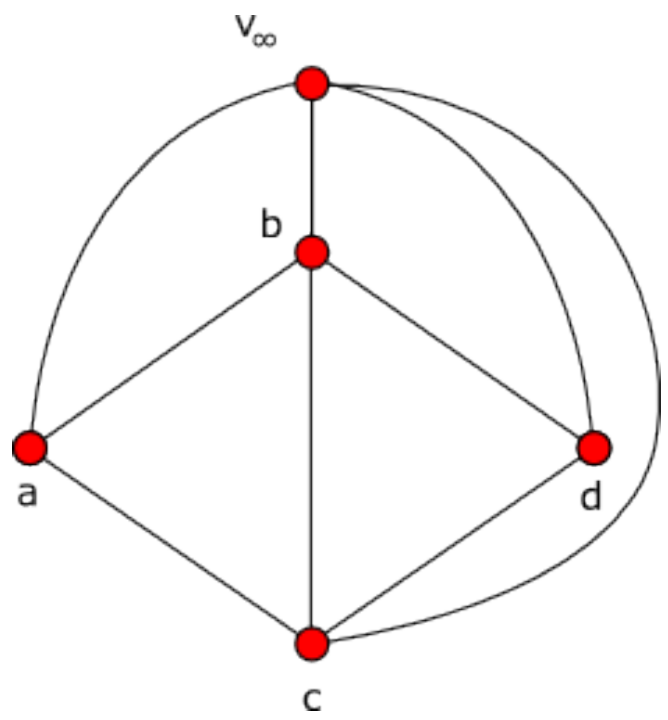

(B)

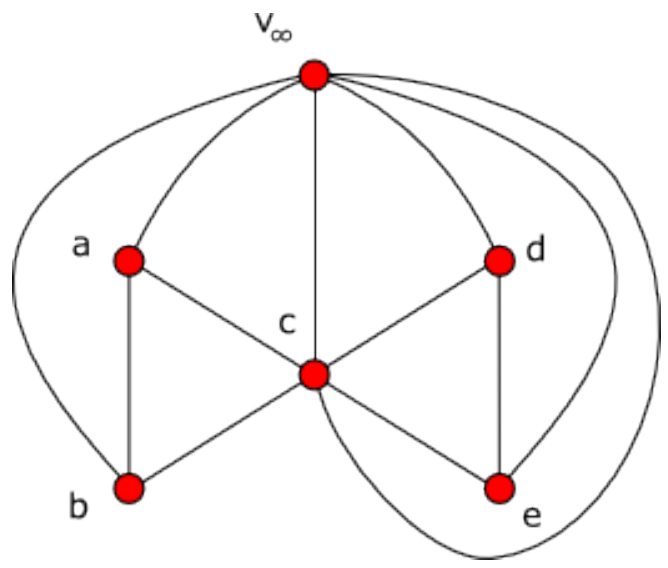

(D)

Figura 7.1: Transformação do grafo plano triangular $\mathbb{T}$ no grafo plano triangulado estendido $\mathbb{G}$. (A) Grafo de Entrada $\mathbb{T}_{1}$. (B) Grafo Plano Triangular $\mathbb{T}_{1}^{\prime}$ com $v_{\infty}$. (C) Grafo de Entrada $\mathbb{T}_{2}$. (D) Grafo Plano Triangular $\mathbb{T}_{2}^{\prime \prime}$ com $v_{\infty}$. de $\mathbb{T}$.

externa. Considere $C$ como sendo o ciclo externo de $\mathbb{T}$. Percorremos o ciclo $C$ e para cada vértice $v$ que visitamos, vamos adicionar uma aresta entre $v$ e $v_{\infty}$ em $\mathbb{G}$ (figura $7.1 \mathrm{~B}$ e figura 7.1D). Note que na figura 7.1D, o ciclo externo passa duas vezes pelo vértice $c$, logo criamos duas arestas $v v_{\infty}$ em $\mathbb{G}$. Chamaremos todas as arestas adicionadas desta forma de arestas de construção,

O último passo da transformação consiste em determinar quais são as faces do grafo que representam os vértices dos cantos do retângulo mais externo da representação retangular. Isso significa que precisamos escolher 4 faces de $\mathbb{G}$ (adjacentes a $v_{\infty}$ ) para dividi-las a fim de criar faces que possuem somente duas arestas. Deste modo, estamos determinando quais são os vértices dos cantos do retângulo mais externo. Note na figura 7.2 que a escolha dos cantos implicam em representações retangulares distintas. A figura 7.3B ilustra um exemplo de escolha que permite a existência de uma representação retangular de $\mathbb{G}$ e a figura $7.3 \mathrm{C}$ 


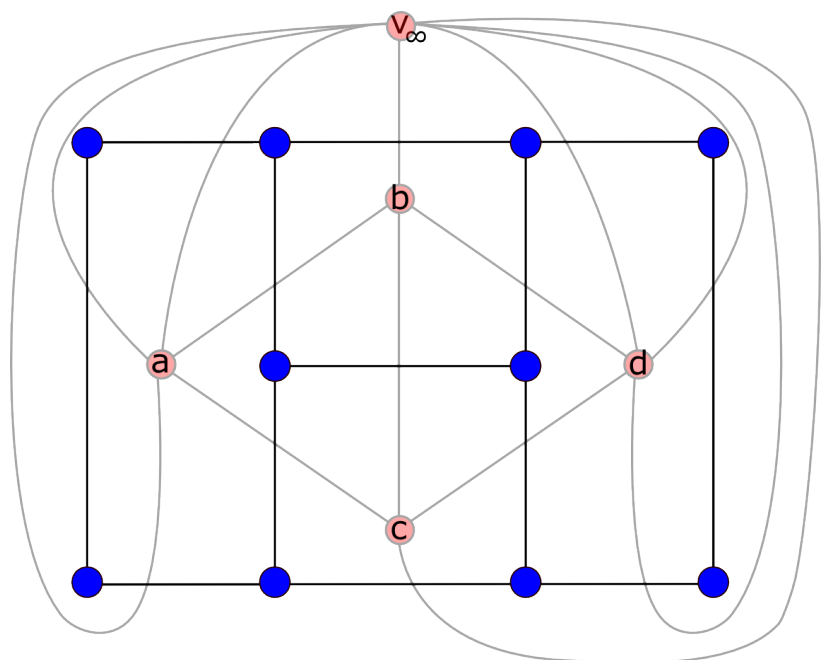

(A)

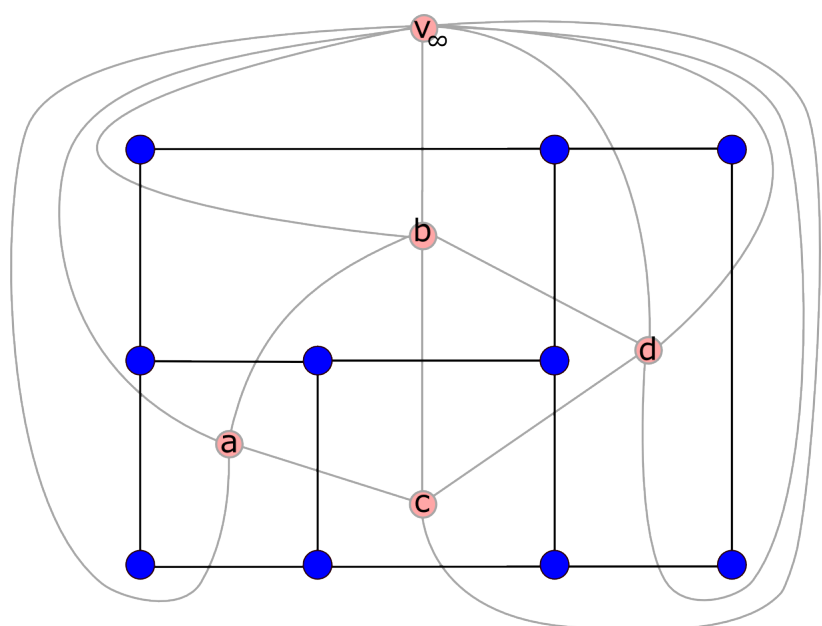

(B)

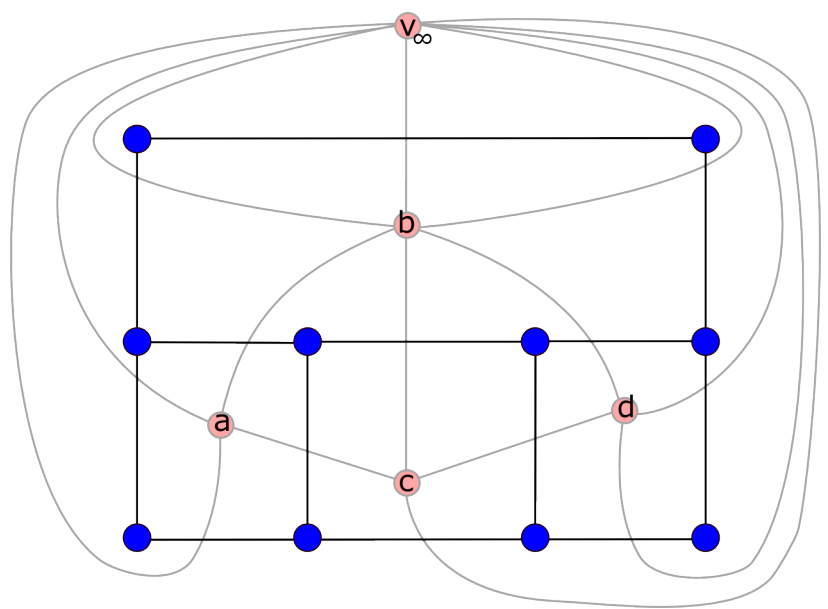

(C)

Figura 7.2: Grafo Plano Triangular Estendido $\mathbb{G}$ e representações retangulares de $\mathbb{T}$.

ilustra um exemplo que a escolha torna inviável a existência de uma representação retangular do grafo $\mathbb{G}$.

Neste momento, vamos supor que fixamos os 4 cantos do retângulo externo e temos um 


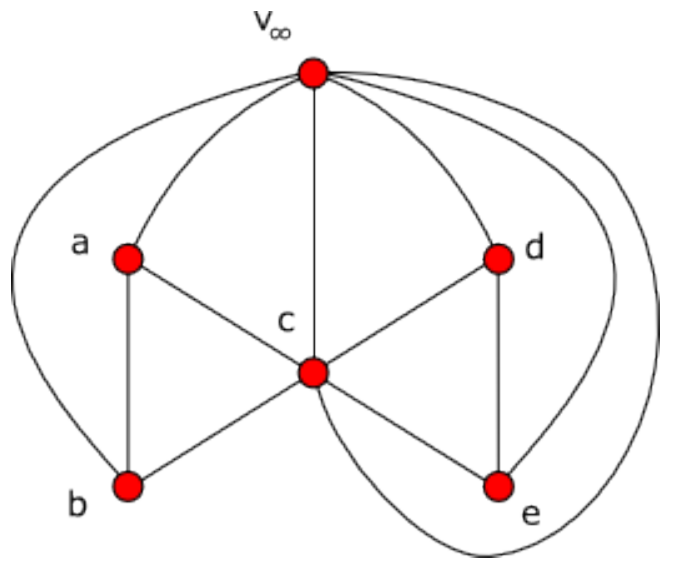

(A)

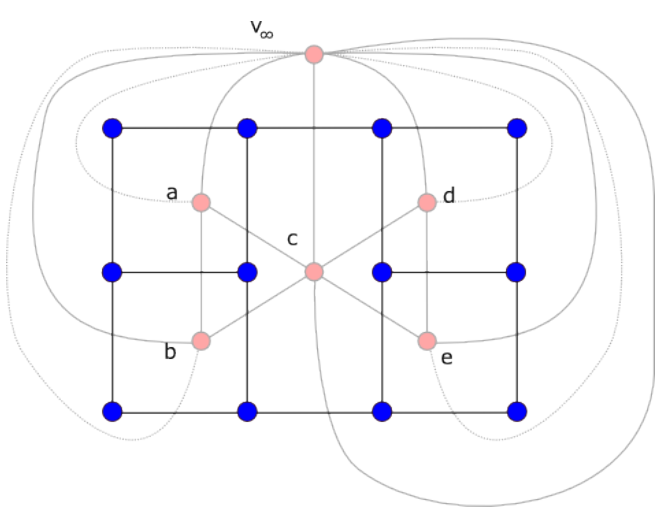

(B)

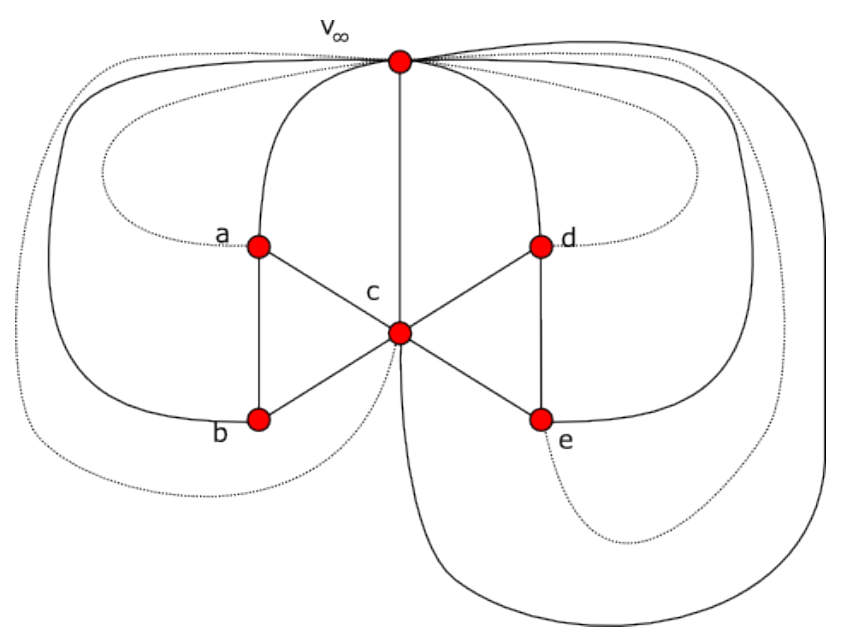

(C)

Figura 7.3: (A) Grafo Plano Triangular. (B) Exemplo de divisão de faces que obtém uma representação retangular do grafo. (C) Exemplo de divisão de faces que torna inviável a existência de uma representação retangular do grafo.

grafo plano triangular estendido $\mathbb{G}$.

Note que todo vértice de um grafo retangular (figura 3.1B) possui grau igual a 2 ou 3. Vamos referenciar um vértice de grau 2 como L-vértice e um vértice de grau 3 como $T$ vértice. Um T-vértice possui dois ângulos de $90^{\circ}$ incidentes a ele e um de $180^{\circ}$. Por outro lado, se olharmos para a face correspondente a este vértice na representação retangular de $\mathbb{G}$, temos uma face formada por 3 arestas e consequentemente temos 3 vértices que pertencem a ela. Vamos dizer que uma face está associada a um vértice se o ângulo de $180^{\circ}$ no grafo retangular está direcionado para ele, como mostrado na figura 7.4.

Seja $\mathbb{G}$ um grafo plano triangular estendido. Para encontrar uma representação retangular de $\mathbb{G}$, precisamos determinar para cada face triangular de $\mathbb{G}$ qual vértice pertencente a ela devemos associá-la. Como os L-vértices da representação retangular não possuem ângulo de $180^{\circ}$ incidente, as faces que possuem duas arestas não serão associadas a nenhum vértice. É conhecido que para qualquer grafo planar, a quantidade de faces que incidem em um vértice $v$ é igual a $d(v)$ (figura 7.5). 

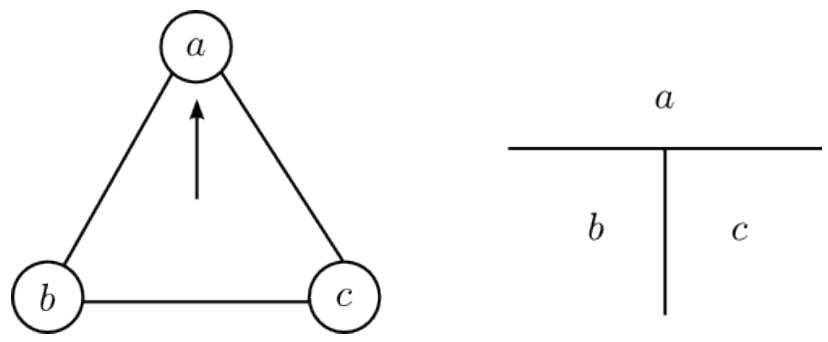

Figura 7.4: Associação da face a um dos seus vértices[LL90]

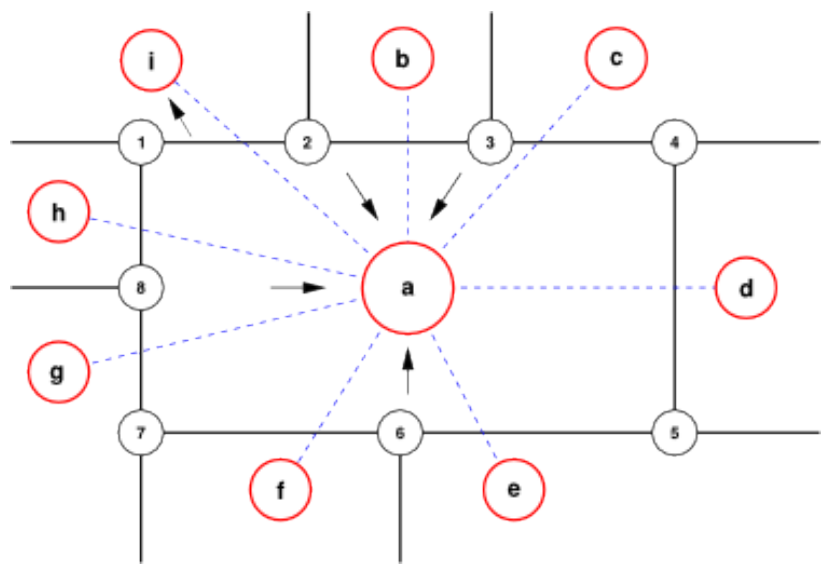

Figura 7.5: Um fragmento de um grafo plano triangular estendido e o fragmento da representação retangular correspondente. [LL90]

A figura 7.5 ilustra o fragmento de um grafo plano triangular estendido e o fragmento da representação retangular referente a este fragmento. Note que as faces 2, 3, 6 e 8 foram associadas ao vértice $a$. Portanto, na representação retangular, os T-vértices destas faces terão o ângulo de $180^{\circ}$ direcionado a $a$. Por outro lado, a face 1 foi associada ao vértice $i$. As associações na figura são representadas pelas setas. Ainda, perceba que o número de faces triangulares associadas a $a$ é igual a $d(a)-4$.

Observando que existe uma relação entre o grau do vértice e a quantidade de faces que devem estar associadas a um vértice de modo que exista uma representação retangular, Lai e Leinwand enunciam os seguintes resultados em [LL90].

Considere um grafo plano $\mathbb{G}$. Seja $\mathbb{G}^{\prime}=\mathbb{G}\left[V^{\prime}\right]$, onde $V^{\prime} \subseteq V(\mathbb{G})$. É fácil ver que existem faces em $\mathbb{G}^{\prime}$ que são limitadas pelas mesmas arestas em $\mathbb{G}$, chamamos estas faces de faces herdadas de $\mathbb{G}$, denotado por $H_{\mathbb{G}}\left(F\left(\mathbb{G}_{S}\right)\right)$. Todas as faces que pertencem ao conjunto $F\left(\mathbb{G}_{S}\right)$ $H_{\mathbb{G}}\left(F\left(\mathbb{G}_{S}\right)\right)$ são chamadas de faces criadas em $\mathbb{G}_{S}$. Ainda, $H_{\mathbb{G}}\left(F_{T}\left(\mathbb{G}_{S}\right)\right)$ denota todas as faces triangulares de $\mathbb{G}_{S}$ que são herdadas de $\mathbb{G}$. 
Lema 7.1: Seja $K=\left(V_{S} ; \overline{V_{S}}\right)$ um corte do grafo plano triangular estendido $\mathbb{G}=$ $(V, E)$. Considere $\mathbb{G}_{S}=\mathbb{G}\left[V_{S}\right]$ o subgrafo de $\mathbb{G}$ induzido por $V_{S}$. Então,

$$
\sum_{v \in V_{S}} d(v)=4\left|V\left(\mathbb{G}_{S}\right)\right|+\left|H_{\mathbb{G}}\left(F_{T}\left(\mathbb{G}_{S}\right)\right)\right|+|K|+2\left|E_{\text {constr }}\right|-\mu_{S}-4
$$

onde $H_{\mathbb{G}}\left(F_{T}\left(\mathbb{G}_{S}\right)\right)$ é o conjunto de faces triangulares herdadas por $\mathbb{G}_{S}$, $E_{\text {constr }}$ é o conjunto das arestas de construção existentes em $\mathbb{G}_{S}$ e $\mu_{S}$ é a quantidade de arestas que pertence à face criada em $\mathbb{G}_{S}$.

Demonstração: Seja $F\left(\mathbb{G}_{S}\right)$ o conjunto das faces existentes em $\mathbb{G}_{S}$. Aplicando a fórmula de Euler, temos:

$$
\left|F\left(\mathbb{G}_{S}\right)\right|=\left|E\left(\mathbb{G}_{S}\right)\right|-\left|V\left(\mathbb{G}_{S}\right)\right|+2 .
$$

Como $K$ é um corte, $\mathbb{G}_{S}$ possui somente uma face que não está definida em $\mathbb{G}$. É fácil ver que esta face pode ser a face externa de $\mathbb{G}_{S}$, pois caso $\mathbb{G}_{S}$ possui um buraco podemos redesenhar $\mathbb{G}_{S}$ de modo que este buraco fique para o lado de fora. Note que esta face não é triangular e que existem $\left|E_{\text {constr }}\right|$ faces limitadas por duas arestas em $\mathbb{G}_{S}$. Logo, a quantidade de faces triangulares existentes em $\mathbb{G}_{S}$ é dada por:

$$
\left|H_{\mathbb{G}}\left(F_{T}\left(\mathbb{G}_{S}\right)\right)\right|=\left|E\left(\mathbb{G}_{S}\right)\right|-\left|V\left(\mathbb{G}_{S}\right)\right|-\left|E_{\text {constr }}\right|+1
$$

Se percorrermos as faces de $\mathbb{G}_{S}$ e contarmos as arestas que pertence a cada face, temos que cada aresta contribui com duas unidades. Logo,

$$
\begin{aligned}
2\left|E\left(\mathbb{G}_{S}\right)\right| & =3\left|H_{\mathbb{G}}\left(F_{T}\left(\mathbb{G}_{S}\right)\right)\right|+2\left|E_{\text {constr }}\right|+\mu_{S} \\
\left|H_{\mathbb{G}}\left(F_{T}\left(\mathbb{G}_{S}\right)\right)\right| & =2\left|E\left(\mathbb{G}_{S}\right)\right|-\mu_{S}-2\left|E_{\text {constr }}\right|-2\left|H_{\mathbb{G}}\left(F_{T}\left(\mathbb{G}_{S}\right)\right)\right|
\end{aligned}
$$

Substituindo,

$$
\left|H_{\mathbb{G}}\left(F_{T}\left(\mathbb{G}_{S}\right)\right)\right|=2\left|V\left(\mathbb{G}_{S}\right)\right|-\mu_{S}-2
$$

Agora, vamos somar o grau de todos os vértices de $\mathbb{G}_{S}$. Como cada aresta de $E\left(\mathbb{G}_{S}\right)$ contribui com 2 e cada aresta de $K$ contribui com 1 temos:

$$
\begin{aligned}
\sum_{v \in V_{S}} & =2\left|E\left(\mathbb{G}_{S}\right)\right|+|K| \\
& =2\left(\left|E\left(\mathbb{G}_{S}\right)\right|-\left|V\left(\mathbb{G}_{S}\right)\right|-\left|E_{\text {constr }}\right|+1\right)-2+2\left|E_{\text {constr }}\right|+2\left|V\left(\mathbb{G}_{S}\right)\right|+|K|
\end{aligned}
$$




$$
\begin{aligned}
& =2\left|H_{\mathbb{G}}\left(F_{T}\left(\mathbb{G}_{S}\right)\right)\right|+2\left|E_{\text {constr }}\right|+2\left|V\left(\mathbb{G}_{S}\right)\right|+|K|-2 \\
& =\left(2\left|V\left(\mathbb{G}_{S}\right)\right|-\mu_{S}-2\right)+\left|H_{\mathbb{G}}\left(F_{T}\left(\mathbb{G}_{S}\right)\right)\right|+2\left|E_{\text {constr }}\right|+2\left|V\left(\mathbb{G}_{S}\right)\right|+|K|-2 \\
& =4\left|V\left(\mathbb{G}_{S}\right)\right|+\left|H_{\mathbb{G}}\left(F_{T}\left(\mathbb{G}_{S}\right)\right)\right|+|K|+2\left|E_{\text {constr }}\right|-\mu_{S}-4 .
\end{aligned}
$$

Teorema 7.2: Seja $\mathbb{G}=(V, E)$ um grafo plano triangular estendido. $\mathbb{G}$ admite uma representação retangular $\mathbb{R}$ se e somente se cada face triangular de $\mathbb{G}$ pode ser associada a um dos vértices que pertencem a ela de modo que cada vértice $v \in V$ esteja associado a exatamente $d(v)-4$ faces triangulares.

Demonstração: Inicialmente, vamos provar a necessidade. Seja $\mathbb{G}$ um grafo plano triangular estendido e $\mathbb{R}$ a representação retangular de $\mathbb{G}$.

Note que para qualquer vértice $v \in V(\mathbb{G})$ o número de faces que incidem em $v$ é igual a $d(v)$ e se observarmos $\mathbb{R}$, com a exceção da face externa, toda face de $\mathbb{R}$ deve possuir quatro ângulos de $90^{\circ}$ em seus cantos.

Seja $f$ uma face que é um retângulo em $\mathbb{R}$. Observe que se um canto é determinado por um T-vértice, então o ângulo de $180^{\circ}$ deve estar direcionado para uma face diferente de $f$. Deste modo, em uma face $f$, existem exatamente quatro T-vértices ou L-vértices de modo que seus ângulos de $90^{\circ}$ estão direcionados para $f$. Ainda, todos os outros T-vértices devem possuir seu ângulo de $180^{\circ}$ direcionados para $f$.

Para o vértice $v_{\infty} \in V(\mathbb{G})$ que representa a face externa $f_{\infty}$ de $\mathbb{R}$, temos um comportamento similar. Todos os ângulos de $180^{\circ}$ dos T-vértices e os ângulos de $270^{\circ}$ dos L-vértices devem estar direcionados para $f_{\infty}$. Note que para cada canto do retângulo externo de $\mathbb{R}$, existe um L-vértice em $\mathbb{G}$, logo, para o vértice $v_{\infty}$, temos que $d\left(v_{\infty}\right)-4$ faces estão associadas a $v_{\infty}$.

Portanto, para qualquer vértice $v \in V(\mathbb{G})$ temos que $d(v)-4$ faces triangulares devem estar associadas a $v$.

Agora, vamos mostrar a suficiência.

Observe que o grafo retangular $\mathbb{R}$ possui todas suas arestas orientadas na horizontal ou vertical. Utilizando dualidade de grafos, é fácil ver que existe uma bijeção entre as arestas do grafo $\mathbb{G}$ e as arestas do grafo da representação retangular $\mathbb{R}$.

Assim, para cada aresta de $\mathbb{G}$ vamos rotulá-la com 0 ou 1. Estes rótulos servirão para definir as orientações das arestas de $\mathbb{R}$. Assuma que as arestas de $\mathbb{G}$ são rotuladas da seguinte forma: 
1. Para cada face triangular $f$ associada a um vértice $v$, as duas arestas de $f$ que incidem em $v$ possuem o mesmo rótulo e a aresta de $f$ que não incide em $v$ possui um rótulo diferente;

2. Para as faces que possuem duas arestas, as arestas devem possuir rótulos distintos.

Como existe uma bijeção entre as arestas de $\mathbb{G}$ e $\mathbb{R}$, o ângulo formado por duas arestas consecutivas de uma face em $\mathbb{G}$ corresponde a um único ângulo em $\mathbb{R}$. Se associarmos as faces de $\mathbb{G}$ a um de seus vértices de modo que satisfaça a hipótese que todo vértice $v$ possui $d(v)-4$ faces associadas a ele, então, em $\mathbb{R}$ toda face $f$, com a exceção da face externa, possui 4 ângulos de $90^{\circ}$ direcionados a $f$. Ainda, as arestas que incidem na face externa $f_{\infty}$ de $\mathbb{R}$ formam um retângulo, pois existem 4 ângulos de $270^{\circ}$ direcionado a $f_{\infty}$.

Vamos mostrar agora que a associação de todas as faces a um dos seus respectivos vértices irá definir as orientações de todas arestas de modo a existir uma representação retangular $\mathbb{R}$. Sabemos que quando estamos associando uma face a um de seus vértices estamos criando uma restrição local dos rótulos das arestas desta face. Devemos provar que estas restrições locais dos rótulos não conflitam e que elas podem ser resolvidas simultaneamente.

Note que dadas todas as associações das faces a um de seus vértices e o rótulo de uma aresta qualquer de $\mathbb{G}$, podemos determinar os rótulos de todas as arestas do grafo $\mathbb{G}$. Seja $K=\left\langle e_{1}, e_{2}, \ldots, e_{i}, e_{i+1}, \ldots, e_{n}\right\rangle$ um corte de $\mathbb{G}$. $K$ define um conjunto de faces $F_{K}=\left\langle f_{1}, f_{2}, \ldots, f_{i}, \ldots, f_{n}\right\rangle$, de modo que $e_{i}$ e $e_{i+1}$ são duas arestas da face $f_{i}$, e as arestas $e_{n}$ e $e_{1}$ são arestas da face $f_{n}$. Claramente, $|K|=\left|F_{K}\right|$.

Suponha que $f_{i}$ é uma face triangular. Denotamos por as $\left(f_{i}\right)$ o vértice a que a face $f_{i}$ está associada, e por $v c\left(f_{i}\right)$ o vértice comum das arestas $e_{i}$ e $e_{i+1}$. As arestas $e_{i}$ e $e_{i+1}$ devem possuir o mesmo rótulo se $v c\left(f_{i}\right)=a s\left(f_{i}\right)$. Caso contrário, devem possuir rótulos diferentes.

Perceba que se o rótulo de uma aresta de $K$ está definido, e as restrições locais das faces de $R_{K}$ não conflitam, então podemos definir os rótulos de todas as arestas das faces de $F_{K}$. Ainda, note que para quaisquer duas faces $f_{1}, f_{2}$ de $\mathbb{G}$ existe um corte, de modo que as faces que são definidas pelas arestas do corte irão conter $f_{1}, f_{2}$. Se mostrarmos para um corte arbitrário $K$ que as restrições locais das faces $F_{K}$ são compatíveis, quaisquer restrições locais associadas a duas faces são compatíveis.

$F_{K}$ pode possuir faces que contêm somente 2 arestas. Considere $F_{K T}$ o subconjunto de $F_{K}$ onde as faces são triangulares e $F_{K D}$ o subconjunto de $F_{K}$ onde as faces são limitadas por duas arestas. Assim, $\left|F_{K}\right|=\left|F_{K T}\right|+\left|F_{K D}\right|$.

Sejam $F_{K N}=\left\{f_{j} \mid f_{j} \in F_{K T} \wedge a s\left(f_{j}\right) \neq v c\left(f_{j}\right)\right\}$. De acordo com as duas regras de rotulação das arestas, os rótulos de duas arestas consecutivas $e_{i}$ e $e_{i+1}$ devem ser diferentes caso $f_{i} \in F_{K D} \cup F_{K N}$. Seja trocas $(K)$ a quantidade de diferenças entre os rótulos de duas arestas consecutivas em $K$, incluindo a comparação entre $e_{n}$ e $e_{1}$. Se $\operatorname{trocas}(K)$ for par, não existe conflito entre as restrições locais definidas por $K$. Como o trocas $(K)$ é dado por 
$\left|F_{K D} \cup F_{K N}\right|$ então precisamos mostrar que $\left|F_{K D} \cup F_{K N}\right|$ sempre é par.

Seja $V_{S}$ e $\overline{V_{S}}$ os dois subconjuntos de $V(\mathbb{G})$ definidos por $K$. Considere $\mathbb{G}\left[V_{S}\right]$ o subgrafo de $\mathbb{G}$ induzido por $V_{S}$. Seja $H_{\mathbb{G}}\left(F_{T}\left(\mathbb{G}_{S}\right)\right)$ o conjunto de faces de $\mathbb{G}_{S}$ herdadas de $\mathbb{G}, \mu_{S}$ a quantidade de arestas que pertence à face criada em $\mathbb{G}_{S}$ e $E_{\text {constr }}$ o conjunto das arestas de construção existentes em $\mathbb{G}_{S}$. Pelo lema 7.1, temos:

$$
\begin{aligned}
& \sum_{v \in V_{S}} d(v)=4\left|V\left(\mathbb{G}_{S}\right)\right|+\left|H_{\mathbb{G}}\left(F_{T}\left(\mathbb{G}_{S}\right)\right)\right|+|K|+2\left|E_{\text {constr }}\right|-\mu_{S}-4 \\
& \sum_{v \in V_{S}} d(v)=4\left|V\left(\mathbb{G}_{S}\right)\right|+\left|H_{\mathbb{G}}\left(F_{T}\left(\mathbb{G}_{S}\right)\right)\right|+\left|F_{K T}\right|+\left|F_{K D}\right|+2\left|E_{\text {constr }}\right|-\mu_{S}-4
\end{aligned}
$$

Desde que cada vértice $v \in V(\mathbb{G})$ possui $d(v)-4$ faces triangulares associadas a ele, o número total de faces associadas aos vértices de $V_{S}$ é dado por:

$$
\sum_{v \in V_{S}}(d(v)-4)=\sum_{v \in V_{S}} d(v)-4\left|V_{S}\right|=\left|H_{\mathbb{G}}\left(F_{T}\left(\mathbb{G}_{S}\right)\right)\right|+\left|F_{K T}\right|+\left|F_{K D}\right|+2\left|E_{\text {constr }}\right|-\mu_{S}-4
$$

As faces herdadas pertencentes a $H_{\mathbb{G}}\left(F_{T}\left(\mathbb{G}_{S}\right)\right)$ devem estar associadas aos vértices de $V_{S}$, pois não existe nenhum vértice $v \in \overline{V_{S}}$ que pertence a uma face de $F_{S T}$. De acordo com a equação anterior, tirando as faces que pertencem a $H_{\mathbb{G}}\left(F_{T}\left(\mathbb{G}_{S}\right)\right)$ existem $\left|F_{K T}\right|+\left|F_{K D}\right|+$ $2\left|E_{\text {constr }}\right|-\mu_{S}-4$ faces associadas aos vértices pertencentes a $V_{S}$. De modo equivalente, temos que a seguinte equação $\mu_{S}-\left|F_{K D}\right|-2\left|E_{\text {constr }}\right|+4$ é a quantidade de faces que não são triangulares e estão associadas a vértices pertencentes a $\overline{V_{S}}$.

Considere:

$$
\begin{aligned}
& F_{x}=\left\{f_{x} \mid f_{x} \in F_{K T} \wedge a s\left(f_{x}\right)=v c\left(f_{x}\right) \wedge v c\left(f_{x}\right) \in\left(\overline{V_{S}}\right)\right\} \\
& F_{y}=\left\{f_{y} \mid f_{y} \in F_{K T} \wedge a s\left(f_{y}\right) \in\left(\overline{V_{S}}\right) \wedge v c\left(f_{y}\right) \in V_{S}\right\} \\
& F_{z}=\left\{f_{z} \mid f_{z} \in F_{K T} \wedge a s\left(f_{z}\right) \in V_{S} \wedge v c\left(f_{z}\right) \in\left(\overline{V_{S}}\right)\right\} .
\end{aligned}
$$

Assim, $F_{x} \cup F_{y}$ é o conjunto de faces triangulares em $F_{K T}$ que estão associadas aos vértices pertencentes a $\overline{V_{S}}$. Então,

$$
\left|F_{x}\right|+\left|F_{y}\right|=\mu_{S}-\left|F_{K D}\right|-2\left|E_{\text {constr }}\right|+4
$$

Uma face $f \in F_{K T}$ tal que duas de suas arestas que pertencem a $K$ e $v c(f) \in \overline{V_{S}}$, deve ter sua terceira aresta pertencendo a $E\left(\mathbb{G}_{S}\right)$. A quantidade destas faces é dada por $\mu_{S}$, então:

$$
\left|F_{x}\right|+\left|F_{z}\right|=\mu_{S}
$$


Assim,

$$
\left|F_{y}\right|+\left|F_{z}\right|=2 \mu_{S}-\left|F_{K D}\right|-2\left|E_{\text {constr }}\right|-2\left|F_{x}\right|+4
$$

Como $F_{K N}=F_{y} \cup F_{z}$, temos:

$$
\left|F_{K N} \cup F_{K D}\right|=\left|F_{y}\right|+\left|F_{z}\right|+\left|F_{K D}\right|=2 \mu_{S}-2\left|E_{\text {constr }}\right|-2\left|F_{x}\right|+4 \text {. }
$$

Portanto, $\left|F_{K N} \cup F_{K D}\right|$ é par.

A construção do grafo plano triangular estendido implica que o grau de cada vértice é maior ou igual a 4 . Seja $\mathbb{G}=(V, E)$ um grafo plano triangular estendido e $W \subseteq V$, define-se $\operatorname{Ex}(W)$ como:

$$
E x(W)=\left(\sum_{v \in W} d(v)-4\right)
$$

Teorema 7.3: Se $\mathbb{G}=(V, E)$ é um grafo plano triangular estendido e $F_{t}(\mathbb{G})$ é o conjunto das faces triangulares, então $\operatorname{Ex}(V)=\left|F_{t}(\mathbb{G})\right|$.

Demonstração: Como a soma dos graus dos vértices de $\mathbb{G}$ é igual ao dobro do número de arestas, temos:

$$
E x(V)=\sum_{v \in V(\mathbb{G})}(d(v)-4)=\sum_{v \in V(\mathbb{G})} d(v)-4|V(\mathbb{G})|=2|E(\mathbb{G})|-4|V(\mathbb{G})| .
$$

Ainda, como cada face triangular de $\mathbb{G}$ possui três arestas e existem quatro faces limitadas por duas arestas em $\mathbb{G}$, a soma de todas as arestas das faces de $\mathbb{G}$ é dada por:

$$
3\left|F_{t}(\mathbb{G})\right|+2 \times 4=2|E(\mathbb{G})|
$$

Pela fórmula de Euler,

$$
|F(\mathbb{G})|=|E(\mathbb{G})|-|V(\mathbb{G})|+2 .
$$

Resultando em,

$$
\begin{aligned}
4\left(\left|F_{t}(\mathbb{G})\right|+4\right) & =4|E(\mathbb{G})|-4|V(\mathbb{G})|+8 \\
\left|F_{t}(\mathbb{G})\right| & =4|E(\mathbb{G})|-4|V(\mathbb{G})|-3\left|F_{t}(\mathbb{G})\right|-8
\end{aligned}
$$




$$
\left|F_{t}(\mathbb{G})\right|=2|E(\mathbb{G})|-4|V(\mathbb{G})|
$$

Pelo Teorema 7.2, qualquer associação das faces aos seus vértices que respeite o limite imposto pelos graus dos vértices resulta em uma representação retangular do grafo. Desta forma, podemos construir um grafo bipartido a partir de um grafo plano triangular estendido $\mathbb{G}$ da seguinte maneira. Considere $G_{b}=\left(X_{b} \cup Y_{b}, E_{b}\right)$ um grafo $\left(X_{b}, Y_{b}\right)$-bipartido, onde:

1. Um vértice $f \in X_{b}$ representa uma face triangular de $\mathbb{G}$.

2. Os vértices pertencentes $Y_{b}$ estão associados aos vértices de $\mathbb{G}$, de modo que para cada vértice $v \in V$ existem $d(v)-4$ 'cópias' de $v$ criadas em $Y_{b}$.

3. Existe uma aresta entre dois vértices $f, v$, onde $f \in X_{b}$ e $v \in Y_{b}$, se e somente se, em $\mathbb{G}, v$ é um vértice pertencente à face representada pelo vértice $f$.

Perceba que o grafo bipartido $G_{b}$ não precisa possuir as $d(v)-4$ 'cópias' de $v_{\infty}$ criadas em $Y_{b}$ nem os vértices em $X_{b}$ correspondente às faces triangulares incidentes em $v_{\infty}$. É fácil ver que para existir um emparelhamento perfeito em $G_{b}$, todos os vértices em $X_{b}$ que representam as faces triangulares que incidem em $v_{\infty}$ (exatamente $d(v)-4$ faces) devem ser emparelhadas com uma cópia de $v_{\infty}$ em $Y_{b}$. Ainda, perceba que pela construção de $G_{b}$, as cópias de $v_{\infty}$ em $Y_{b}$ e aos vértices de $X_{b}$ que representam as faces triangulares incidentes em $v_{\infty}$ formam uma biclique. Outro fato importante é que qualquer emparelhamento perfeito nesta biclique não altera a representação retangular. Logo, nas figuras a seguir iremos omitir as cópias de $v_{\infty}$ em $Y_{b}$ e os vértices em $X_{b}$ que representam as faces triangulares incidentes em $v_{\infty}$

A figura 7.6B ilustra o grafo bipartido construído a partir do grafo da figura 7.6A. Note que um emparelhamento perfeito do grafo bipartido associa cada face a um vértice que pertence a ela e que cada vértice $v$ de $\mathbb{G}$ possui $d(v)-4$ faces associadas a ele.

Teorema 7.4: Um grafo plano triangular estendido $G$ admite uma representação retangular se e somente se existe um emparelhamento perfeito no grafo bipartido construído a partir de $\mathbb{G}$.

Demonstração: Este teorema é consequência dos teoremas 7.2 e 7.3.

Podemos visualizar o resultado das associações das faces aos vértices na figura 7.7A e a respectiva representação retangular na figura 7.7B. O Teorema 7.4 permite a construção de um algoritmo polinomial para determinar, dado um grafo plano triangular $\mathbb{T}$, se existe ou não uma representação retangular do grafo $\mathbb{T}$. 


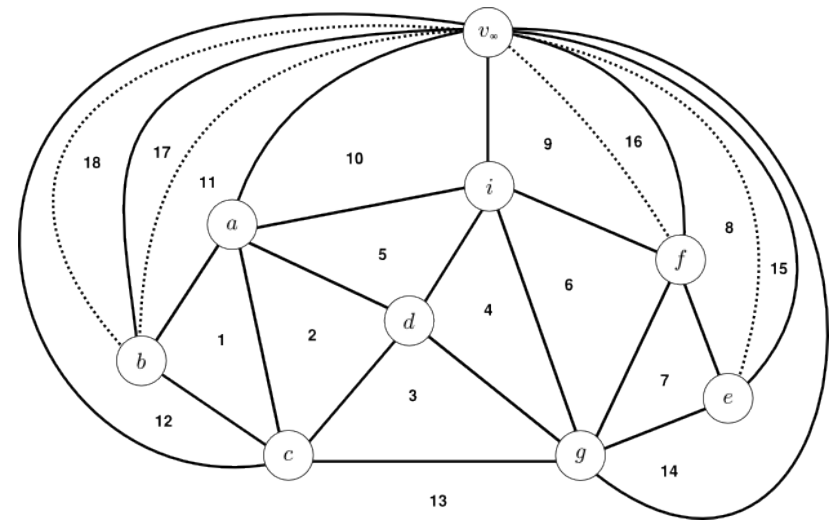

(A)

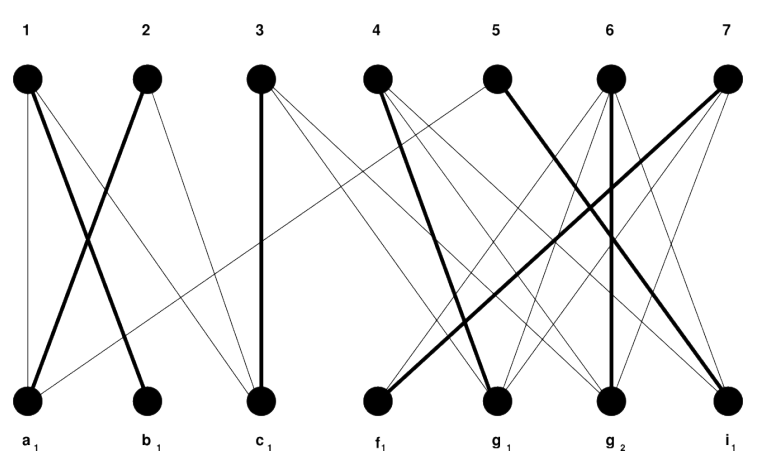

(B)

Figura 7.6: (A) Grafo Plano Triangular Estendido $\mathbb{G}$. (B) Grafo bipartido $G_{b}$ construído a partir de $\mathbb{G}$ e um emparelhamento perfeito de $G_{b}$ representado pelas arestas mais grossas.[LL90]

O algoritmo consiste em escolher quatro vértices de $V(\mathbb{T})$ para serem os cantos da representação retangular e transformar $\mathbb{T}$ em um grafo plano triangular estendido $\mathbb{G}$ e construir, como indicado, um grafo bipartido $G_{b}$ a partir de $\mathbb{G}$. Devemos verificar se existe ou não um emparelhamento perfeito em $G_{b}$. Existem diversos algoritmos para solucionar este problema. O método húngaro [Fra05] é simples e possui complexidade computacional $O\left(n^{3}\right)$. Podemos utilizar o algoritmo de Hopcroft e Karp [HK73] que possui complexidade computacional $O\left(n^{5 / 2}\right)$. Assim, mesmo testando todas as possibilidades dos quatro cantos da representação retangular o método apresentado por Lai e Leinwand ainda é polinomial.

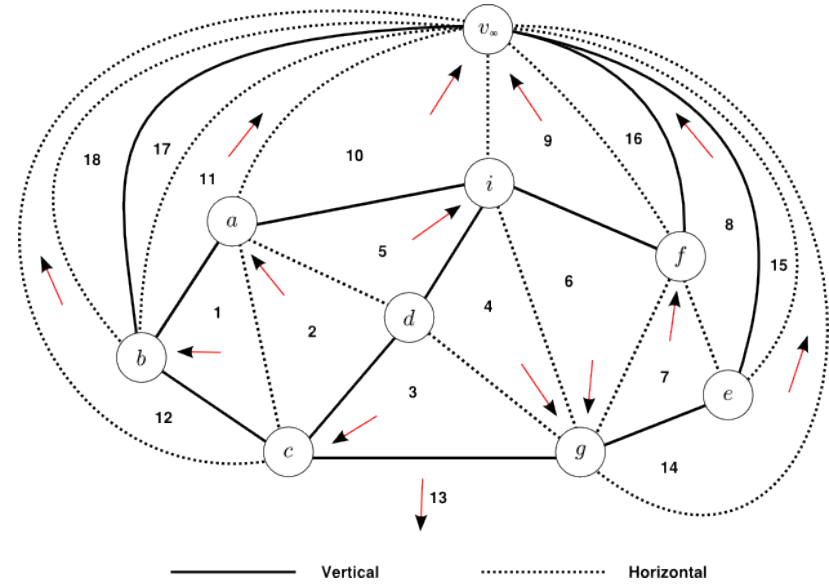

(A)

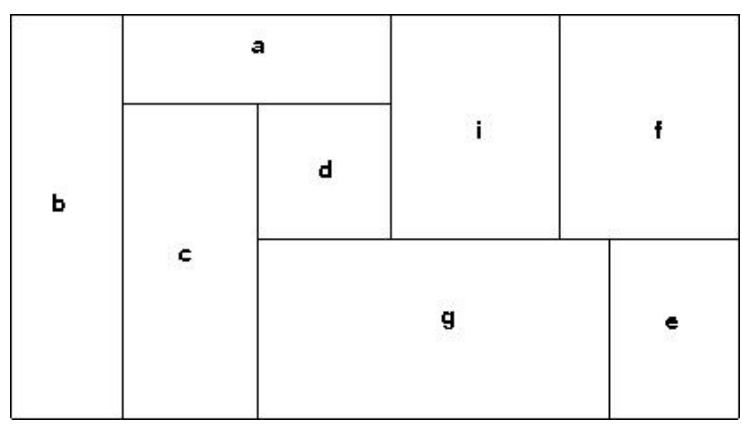

(B)

Figura 7.7: (A) Uma associação perfeita das faces triangulares. (B) Representação retangular resultante da associação de (A) [LL90]. 


\subsection{REDUÇÃO DO PROBLEMA DE ENCONTRAR UMA REPRESENTAÇÃO RETANGULAR SEM A NECESSIDADE DE DETERMINAR OS CANTOS DA REPRESENTAÇÃO}

Como já apresentado anteriormente, não podemos transformar um grafo plano triangular $\mathbb{T}$ em um grafo plano triangular estendido $\mathbb{G}$ arbitrariamente. Agora, vamos mostrar como modificar a construção do grafo bipartido $G_{b}$ apresentada anteriormente por Lai e Leinwand para encontrar uma representação retangular de um grafo plano triangular $\mathbb{T}$ sem precisar testar todas as possibilidades dos vértices que serão os cantos da representação retangular.

De modo análogo ao apresentado anteriormente, considere o grafo plano triangular $\mathbb{G}$ que é uma cópia de $\mathbb{T}$ juntamente com o vértice $v_{\infty}$ e as arestas entre o vértice $v_{\infty}$ e todos os vértices que incidem na face externa de $\mathbb{T}$. Assim, todos os vértice neste novo grafo deve possui grau maior ou igual a 3. Para que um grafo admita uma representação retangular, todos os vértices além de, no máximo, 4 vértices adjacentes a $v_{\infty}$ devem possuir grau maior ou igual a 4 . Logo, se existe algum vértice $u$ que possua grau 3 devemos adicionar uma aresta $u v_{\infty}$ a $\mathbb{T}$. Note que estes vértices serão cantos da representação retangular de $\mathbb{T}$.

É fácil ver que se existe algum vértice com grau igual a 3 este deve ser adjacente a $v_{\infty}$. Senão isso contrariaria a suposição de $\mathbb{T}$ ser um grafo plano triangulado. Considere $\eta$ a quantidade de vértices em $\mathbb{G}$ adjacentes a $v_{\infty}$ com grau 3.

Deste modo, gostaríamos de construir um grafo bipartido semelhante ao construído quando possuímos um grafo plano triangular estendido. É fácil notar que, para qualquer aresta que adicionarmos em $\mathbb{G}$ iremos criar uma face que contém somente duas arestas, e como argumentado anteriormente, esta face irá representar um canto do retângulo mais externo da representação retangular. Assim, para cada vértice $v$ de grau 3, vamos adicionar uma aresta entre $v$ e $v_{\infty}$. Assim, determinamos $\eta$ cantos, restando escolher, $4-\eta$ cantos. Assim, gostaríamos de construir um grafo bipartido de modo que um emparelhamento perfeito, além de associar as faces aos vértices, determine os cantos que ainda não foram definidos.

Para isso, vamos construir um grafo $\left(X_{b}^{\prime}, Y_{b}^{\prime}\right)$-bipartido $G_{b}^{\prime}=\left(X_{b}^{\prime} \cup Y_{b}^{\prime}, E_{b}^{\prime}\right)$ a partir de $\mathbb{G}$. $G_{b}^{\prime}$ é definido da seguinte forma:

1. Cada vértice em $X_{b}^{\prime}$ representa uma face triangular de $\mathbb{G}$ que não incide em $v_{\infty}$.

2. $Y_{b}^{\prime}$ é composto por dois subconjuntos: $Y_{b e}^{\prime}$ e $Y_{b c}^{\prime}$. Os vértices em $Y_{b e}^{\prime}$ são associados aos vértices de $\mathbb{G}$, de modo que existem $d\left(v_{i}\right)-4$ vértices criados em $Y_{b e}^{\prime}$ para cada vértice $v_{i} \in V(\mathbb{G}) \backslash\left\{v_{\infty}\right\}$ de modo que $d\left(v_{i}\right) \geq 4$. $Y_{b c}^{\prime}$ é um conjunto que contém $4-\eta$ vértices. 
3. Existe uma aresta entre dois vértices $f, v$, onde $f \in X_{b}^{\prime}$ e $v \in Y_{b e}^{\prime}$, se e somente se a face representada pelo vértice $f$ incide no vértice $v$ em $\mathbb{G}$. Para cada p vértice $f_{i} \in X_{b}^{\prime}$ existe uma aresta conectando $f_{i}$ a cada um dos vértices em $Y_{b c}^{\prime}$ se $f_{i}$ representa uma face de $\mathbb{T}$ que incide em ao menos um vértice que é adjacente a $v_{\infty}$.

A figura 7.8 ilustra esta construção e apresenta uma representação retangular a partir do emparelhamento perfeito apresentado.

Em um emparelhamento perfeito $E$ de $G_{b}^{\prime}$, temos um conjunto $W$ vértices que estão associados aos vértices que pertencem a $Y_{b}^{\prime}$. Pela cardinalidade de $Y_{b}^{\prime}$ temos que $|W|=4-\eta$. Pela construção de $G_{b}^{\prime}$ cada vértice em $W$ representa uma face triangular que incide em ao menos um vértice adjacente a $v_{\infty}$. Agora, vamos analisar o que isso representa no grafo $\mathbb{T}$. Seja $F$ o conjunto das faces triangulares de $\mathbb{G}$ representadas pelos vértices de $W$. Temos que associar cada face $f$ de $F$ a um dos vértices que são adjacentes a $v_{\infty}$, denominamos este por $v_{f}$. Depois de atribuirmos todas as faces de $F$, para cada vértice $v_{f}$ de modo que $f \in F$, vamos adicionar uma aresta de construção, ou seja, uma aresta de $v_{f}$ a $v_{\infty}$.

Note que na figura 7.8C os vértices $a$ e $b$ recebem uma aresta de construção e $e$ recebe duas arestas de construção. Pelos argumentos apresentados anteriormente, precisamos criar para cada vértice de grau 3 uma aresta de construção ( $b$ e $e$ ). Pela construção apresentada no parágrafo anterior, precisamos adicionar uma aresta para cada vértice $v_{f}$, onde $f \in F$ ( $a$ e $e$ ).

\subsection{CARACterizAÇÃo DE LAi E LeinWAND PARA O PROBLEMA DA REPRESENTAÇÃO RETANGULAR}

A partir da redução do problema de encontrar uma representação retangular a encontrar um emparelhamento perfeito em um grafo bipartido, podemos enunciar algumas condições necessárias e suficientes para a existência de tais representação retangulares. Lai e Leinwand [LL90] reescrevem o teorema de Hall [BM08].

Teorema 7.5: Considere $\mathbb{G}=(V, E)$ um grafo plano triangular estendido e $G_{b}=$ $\left(X_{b} \cup Y_{b}, E_{b}\right)$ o grafo bipartido construído a partir de $\mathbb{G}$. Existe um emparelhamento perfeito $M$ em $G_{b}$ se e somente se

1.todo subconjunto $X$ de $X_{b}$ (vértices que representam faces triangulares) é adjacente a um subconjunto $Y$ de $Y_{b}$ de modo que $|X| \leq|Y|$, ou

2.todo subconjunto $Y$ de $Y_{b}$ é adjacente a um subconjunto $X$ de $X_{b}$ de modo que $|Y| \leq|X|$. 


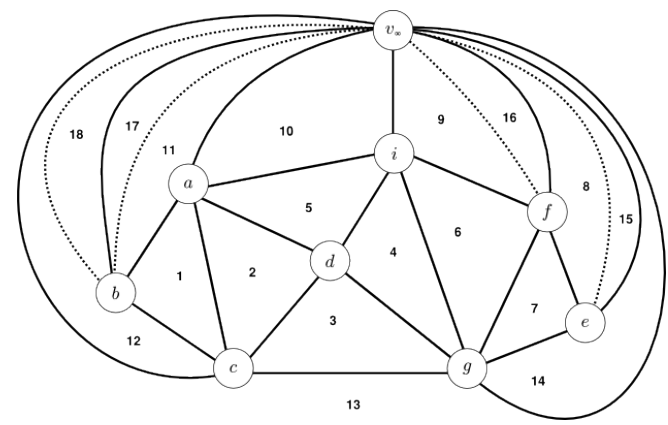

(A)

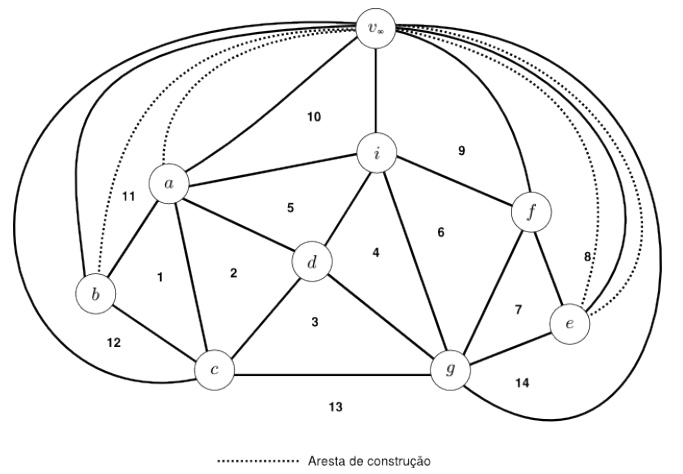

(C)

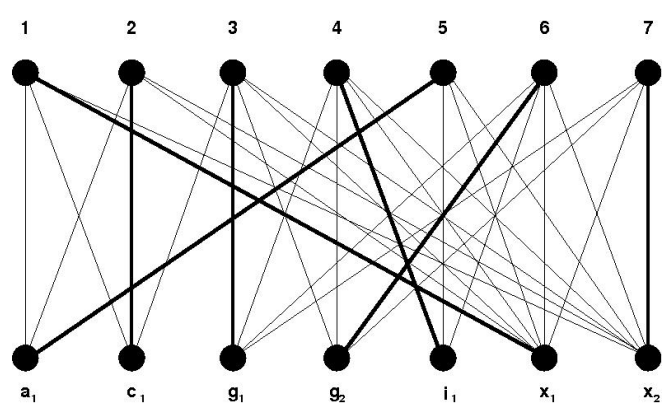

(B)

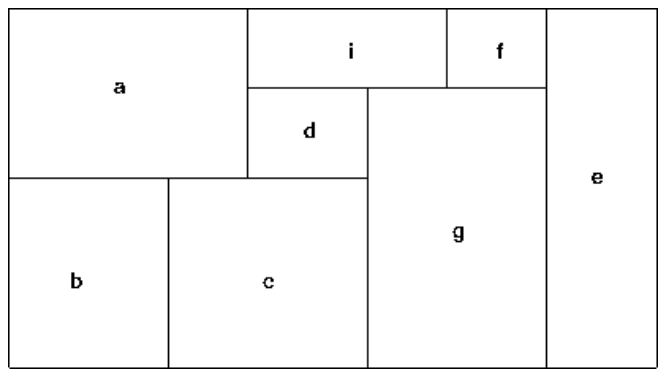

(D)

Figura 7.8: (A) Grafo Plano Triangular $\mathbb{G}$. (B) Grafo bipartido construído a partir de $\mathbb{G}$ e em negrito uma associação perfeita das faces triangulares. (C) Ilustração das associações no grafo $\mathbb{G}$. (D) Representação retangular resultante da associação de (B) [LL90].

Observe que o teorema 7.5 é um resultado para o problema de encontrar um emparelhamento perfeito em grafos bipartidos, entretanto, no enunciado do teorema 7.5 não descrevemos nenhuma propriedade do grafo plano triangular estendido $\mathbb{G}$. Assim, a seguir iremos apresentar uma interpretação deste resultado caracterizando uma propriedade no grafo plano triangular estendido $\mathbb{G}$.

Seja $\mathbb{G}=(V, E)$ um grafo plano triangular estendido e $G_{b}=\left(X_{b} \cup Y_{b}, E_{b}\right)$ o grafo bipartido construído a partir de $\mathbb{G}$. Considere $V_{S} \subseteq V(\mathbb{G})$, denotamos por $R I\left(V_{S}\right)$ o subconjunto de faces triangulares de $\mathbb{G}$ de modo que uma face triangular $f$ pertence a $R I\left(V_{S}\right)$ se $f$ incide em algum vértice de $V_{S}$.

Seja $V_{S} \subseteq V(\mathbb{G})$ e $Y_{S}$ todos os vértices de $Y_{b}$ que estão associados aos vértices de $V_{S}$. Como para cada vértice $v \in V_{S}$, existem $d(v)-4$ vértices associados a $v$ em $Y_{b}$, então $\left|Y_{S}\right|=\operatorname{Ex}\left(V_{S}\right)$. Ainda, a quantidade de vértices em $X_{b}$ adjacentes a $Y_{b}$ é igual a $\left|R I\left(V_{S}\right)\right|$. 
Teorema 7.6: Um grafo plano triangular estendido $\mathbb{G}$ admite uma representação retangular se e somente se para cada corte $\left(V_{S} ; \overline{V_{S}}\right)$ de $\mathbb{G}, \operatorname{Ex}\left(V_{S}\right) \leq\left|R I\left(V_{S}\right)\right|$.

Demonstração: Inicialmente, vamos provar a necessidade.

Considere o grafo bipartido $G_{b}=\left(X_{b} \cup Y_{b}, E_{b}\right)$ construído a partir de $\mathbb{G}$. Note que pela hipótese existe uma representação retangular de $\mathbb{G}$, então, pelo teorema 7.5 temos que $\operatorname{Ex}\left(V_{S}\right) \leq\left|R I\left(V_{S}\right)\right|$.

Para provar a suficiência, considere que $F_{T S}$ é um subconjunto de $F_{T}(\mathbb{G})$ de modo que o subgrafo de $\mathbb{G}$ induzido pelas arestas das faces de $F_{T S}$ é conexo. Assim, o subconjunto $V(\mathbb{G}) \backslash V\left(F_{T S}\right)$ pode ser dividido em $m$ subconjuntos disjuntos: $V_{1}, V_{2}, \ldots, V_{m}$, de forma que $\left(V_{1} ; \overline{V_{1}}\right),\left(V_{2} ; \overline{V_{2}}\right), \ldots,\left(V_{m} ; \overline{V_{m}}\right)$ são cortes disjuntos nas arestas. Ademais, os conjuntos $R I\left(V_{1}\right), R I\left(V_{2}\right), \ldots, R I\left(V_{m}\right)$ são dois a dois disjuntos. Note que nenhuma face que pertencem a $F_{T S}$ incide em algum vértice pertencente a $V(\mathbb{G}) \backslash V\left(F_{T S}\right)$. Logo,

$$
F_{T S} \cap R I\left(V_{i}\right)=\emptyset \quad \text { para } \mathrm{i} \leq \mathrm{i} \leq \mathrm{m} .
$$

Como pode existir uma face triangular que incide em algum vértice de $V\left(F_{T S}\right)$, mas não pertence a $F_{T S}$, temos:

$$
\left|F_{T S}\right| \leq\left|F_{T}(\mathbb{G})\right|-\left(\left|R I\left(V_{1}\right)\right|+\left|R I\left(V_{2}\right)\right|+\ldots+\left|R I\left(V_{m}\right)\right|\right) .
$$

Como $V\left(F_{T S}\right), V_{1}, V_{2}, \ldots, V_{m}$ são dois a dois disjuntos, temos que $V\left(F_{T S}\right) \cup V_{1} \cup V_{2} \cup \ldots \cup$ $V_{m}=V(\mathbb{G})$. Logo,

$$
\operatorname{Ex}(V(\mathbb{G}))=\operatorname{Ex}\left(V\left(F_{T S}\right)\right)+E x\left(V_{1}\right)+E x\left(V_{2}\right)+\ldots+E x\left(V_{m}\right) .
$$

Pelo teorema 7.3, temos que $\left|F_{T}(\mathbb{G})\right|=\operatorname{Ex}(V(\mathbb{G}))$.

$$
\begin{aligned}
F_{T S} \leq & \operatorname{Ex}\left(V\left(F_{T S}\right)\right)+\operatorname{Ex}\left(V_{1}\right)+\operatorname{Ex}\left(V_{2}\right)+\ldots+\operatorname{Ex}\left(V_{m}\right) \\
& -\left(\left|R I\left(V_{1}\right)\right|+\left|R I\left(V_{2}\right)\right|+\ldots+\left|R I\left(V_{m}\right)\right|\right) .
\end{aligned}
$$

Pela hipótese,

$$
\begin{array}{r}
\operatorname{Ex}\left(V_{i}\right) \leq\left|R I\left(V_{i}\right)\right| \quad \text { para } \mathrm{i} \leq \mathrm{i} \leq \mathrm{m}, \\
\left|F_{T S}\right| \leq \operatorname{Ex}\left(V\left(F_{T S}\right)\right)
\end{array}
$$


Agora, vamos relacionar este resultado com o grafo bipartido $G_{b}=\left(X_{b} \cup Y_{b}, E_{b}\right)$ construído a partir de $\mathbb{G}$.

Seja $X_{K} \subseteq X_{b}$ e $F_{K}$ o conjunto das faces triangulares de $\mathbb{G}$ que estão associadas aos vértices de $X_{K}$. Pela construção de $G_{b}$, temos que existe uma bijeção entre as faces triangulares de $\mathbb{G}$ e os vértices de $X_{b}$. Assim,

$$
\left|F_{K}\right|=\left|X_{K}\right|
$$

Vamos particionar $F_{K}$ em $n$ subconjuntos: $F_{1}, F_{2}, \ldots, F_{n}$ onde para cada subconjunto $F_{i}, 1 \leq i \leq n$, temos que o subgrafo de $\mathbb{G}$ induzido pelas arestas de $F_{i}$ é conexo. De modo análogo ao apresentado anteriormente, temos

$$
\left|F_{i}\right| \leq \operatorname{Ex}\left(V\left(F_{i}\right)\right)
$$

Ainda, como $V\left(F_{1}\right), V\left(F_{2}\right), \ldots, V\left(F_{n}\right)$ são dois a dois disjuntos.

$$
\left|F_{K}\right|=\sum_{i=1}^{n}\left|F_{i}\right| \leq \sum_{i=1}^{n} \operatorname{Ex}\left(V\left(F_{i}\right)\right)=\operatorname{Ex}\left(V\left(F_{K}\right)\right)
$$

Portanto, o tamanho do subconjunto de vértices em $Y_{b}$ adjacentes aos vértices de $X_{K}$ é igual a $E x\left(V\left(F_{K}\right)\right)$. O que implica que para qualquer subconjunto de $X_{b}$ temos que a primeira condição do teorema 7.5 é satisfeita. Logo, existe um emparelhamento perfeito em $G_{b}$ e pelo teorema $7.4 \mathbb{G}$ possui uma representação retangular.

Os próximos lemas serão necessários para provar o teorema 7.10. O teorema 7.10 é um resultado equivalente aos mostrados nos capítulos 4 e 5 .

Lema 7.7: Seja $K=\left(V_{S} ; \overline{V_{S}}\right)$ um corte do grafo plano triangular estendido $\mathbb{G}=$ $(V, E)$. Considere $\mathbb{G}_{S}=\mathbb{G}\left[V_{S}\right]$ o subgrafo de $\mathbb{G}$ induzido por $V_{S}$. Então,

$$
\operatorname{Ex}\left(V_{S}\right)=\left|R I\left(V_{S}\right)\right|+2\left|E_{\text {constr }}^{\mathbb{G}_{S}}\right|+\left|E_{\text {constr }}^{K}\right|-\mu_{S}-4,
$$

onde $E_{\text {constr }}^{\mathbb{G}_{S}}$ é o conjunto das arestas de construção existente em $\mathbb{G}_{S}, E_{\text {constr }}^{K}$ é o conjunto das arestas de construção existente em $K$ e $\mu_{S}$ é a quantidade de arestas que pertence à face criada em $\mathbb{G}_{S}$.

Demonstração: Temos pelo lema 7.1,

$$
\sum_{v \in V_{S}}(d(v)-4)=\left|H_{\mathbb{G}}\left(F_{T}\left(\mathbb{G}_{S}\right)\right)\right|+|K|+2\left|E_{\text {constr }}^{\mathbb{G}_{S}}\right|-\mu_{S}-4
$$


Considere $F_{K}$ o conjunto de faces que possuem ao menos uma aresta de corte $K$. Considere $F_{K T}$ as faces triangulares de $F_{K}$ e $F_{K D}$ as faces limitadas por duas arestas. Assim,

$$
\begin{aligned}
\left|F_{K D}\right| & =\left|E_{\text {constr }}^{K}\right| \\
|K| & =\left|F_{K T}\right|+\left|E_{\text {constr }}^{K}\right| .
\end{aligned}
$$

Agora, vamos contar a quantidade de faces triangulares que incidem em $V_{S}$. Além das faces triangulares herdadas $H_{\mathbb{G}}\left(F_{T}\left(\mathbb{G}_{S}\right)\right)$, temos as faces $F_{K T}$. Logo,

$$
R I\left(V_{S}\right)=H_{\mathbb{G}}\left(F_{T}\left(\mathbb{G}_{S}\right)\right) \cup F_{K T}
$$

Portanto,

$$
\operatorname{Ex}\left(V_{S}\right)=\left|R I\left(V_{S}\right)\right|+2\left|E_{\text {constr }}^{\mathbb{G}_{S}}\right|+\left|E_{\text {constr }}^{K}\right|-\mu_{S}-4
$$

Lema 7.8: Um grafo plano triangular estendido $\mathbb{G}$ possui uma representação retangular se satisfaz as seguintes condições:

1.Nenhum vértice adjacente a $v_{\infty}$ possui mais de duas arestas de construção;

2.Se $|V(\mathbb{G})|>3 \mid$ e somente dois vértices adjacentes a $v_{\infty}$ possuem arestas de construção, então não pode existir uma aresta entre estes dois vértices.

Demonstração: Vamos provar as duas propriedades separadamente.

Para (1), considere um grafo plano triangular estendido $\mathbb{G}_{1}$ tal que $V\left(\mathbb{G}_{1}\right) \geq 3$.

Suponha por absurdo que exite um vértice $v_{1} \in V\left(\mathbb{G}_{1}\right)$ tal que $v_{1}$ possui mais que duas arestas de construção. Seja $V^{\prime}=\left\{v_{\infty}, v_{1}\right\}$ e $\mathbb{G}^{\prime}=\mathbb{G}_{1}\left[V^{\prime}\right]$. Note que a face criada em $\mathbb{G}^{\prime}$ é limitada por duas arestas e exitem mais que duas arestas de construção em $E\left(\mathbb{G}^{\prime}\right)$.

Como $v_{\infty} \in V\left(\mathbb{G}^{\prime}\right)$, ou as 4 arestas de construção pertencem a $E\left(\mathbb{G}^{\prime}\right)$ ou pertencem ao corte $K=\left(V^{\prime} ; \overline{V^{\prime}}\right)$ em $\mathbb{G}_{1}$. Pelo lema 7.7 , temos:

$$
\begin{aligned}
& E x\left(V^{\prime}\right)=\left|R I\left(V^{\prime}\right)\right|+2\left|E_{\text {constr }}^{\mathbb{G}^{\prime}}\right|+\left|E_{\text {constr }}^{K}\right|-\mu_{S}-4 \\
& E x\left(V^{\prime}\right)=\left|R I\left(V^{\prime}\right)\right|+2\left|E_{\text {constr }}^{\mathbb{G}^{\prime}}\right|+\left|E_{\text {constr }}^{K}\right|-6 .
\end{aligned}
$$

Ainda,

$$
\left|E_{\text {constr }}^{\mathbb{G}^{\prime}}\right|+\left|E_{\text {constr }}^{K}\right|=4
$$




$$
2\left|E_{\text {constr }}^{\mathbb{G}^{\prime}}\right| \geq 2 * 3=6
$$

Assim,

$$
2\left|E_{\text {constr }}^{\mathbb{G}^{\prime}}\right|+\left|E_{\text {constr }}^{K}\right|>6
$$

Logo,

$$
\operatorname{Ex}\left(V^{\prime}\right)>\left|R I\left(V^{\prime}\right)\right|
$$

Um absurdo, pois pelo teorema 7.6, $\mathbb{G}_{1}$ não possui uma representação retangular.

Agora vamos provar (2). Considere um grafo plano triangular estendido $\mathbb{G}_{2}$ tal que $V\left(\mathbb{G}_{2}\right) \geq 4$. Assuma que existem somente dois vértices $u, v \in V\left(\mathbb{G}_{2}\right)$ que possuem arestas de construção e, por absurdo, $u$ é adjacente a $v$. Seja $V^{\prime}=\left\{v_{\infty}, u, v\right\}$ e $\mathbb{G}^{\prime}=\mathbb{G}\left[V^{\prime}\right]$. Note que a face criada em $\mathbb{G}^{\prime}$ é limitada por três arestas e que existem quatro arestas de construção em $E\left(\mathbb{G}^{\prime}\right)$. Logo, pelo lema 7.7, temos:

$$
\begin{aligned}
& E x\left(V^{\prime}\right)=\left|R I\left(V^{\prime}\right)\right|+2\left|E_{\text {constr }}^{\mathbb{G}^{\prime}}\right|+\left|E_{\text {constr }}^{K}\right|-\mu_{S}-4 \\
& E x\left(V^{\prime}\right)=\left|R I\left(V^{\prime}\right)\right|+2 * 4+0-3-4 \\
& E x\left(V^{\prime}\right)=\left|R I\left(V^{\prime}\right)\right|+1 .
\end{aligned}
$$

Um absurdo, pois pelo teorema 7.6, $\mathbb{G}_{2}$ não possui uma representação retangular.

O lema 7.8 mostra que uma face não pode conter mais de dois cantos do retângulo mais externo da representação retangular. Ainda, também mostra que duas faces que são adjacentes não podem conter todos os cantos do retângulo mais externo da representação retangular a não ser se estas são as únicas faces internas da representação retangular.

Lema 7.9: Seja $\mathbb{G}$ um grafo plano triangular estendido que satisfaz o lema 7.8. Seja $K=\left(V_{S} ; \overline{V_{S}}\right)$ um corte de $\mathbb{G}$. Considere $\mathbb{G}_{S}=\mathbb{G}\left[V_{S}\right]$ o subgrafo de $\mathbb{G}$ induzido por $V_{S}$. Se $\left|V\left(\mathbb{G}_{S}\right)\right|=2$ ou existe um vértice $v \in V\left(\mathbb{G}_{S}\right)$ de modo que $\mathbb{G}_{S}-v$ não é conexo, então $\operatorname{Ex}\left(V_{S}\right) \leq R I\left(V_{S}\right)$.

Demonstração: Se existe um vértice $v \in V\left(\mathbb{G}_{S}\right)$ de modo que $\mathbb{G}_{S}-v$ não é conexo, então $\mathbb{G}_{S}$ possui mais de um bloco e a face criada em $\mathbb{G}_{S}$ é limitada por pelo menos quatro arestas. Como existem exatamente quatro arestas de construção, então pelo lema $7.7 \operatorname{Ex}\left(V\left(\mathbb{G}_{S}\right)\right) \leq$ $\left|R I\left(V\left(\mathbb{G}_{S}\right)\right)\right|$.

Caso $V\left(\mathbb{G}_{S}\right)=2$, então a face criada em $\mathbb{G}_{S}$ é limitada por duas arestas. Como $\mathbb{G}$ satisfaz 
as condições do lema 7.8, então $\left|E_{\text {constr }}^{\mathbb{G}_{S}}\right| \leq 2$. Implicando que $2\left|E_{\text {constr }}^{\mathbb{G}_{S}}\right|+\left|E_{\text {constr }}^{K}\right| \leq 6$. Portanto, pelo lema 7.7, $\operatorname{Ex}\left(V\left(\mathbb{G}_{S}\right)\right) \leq\left|R I\left(V\left(\mathbb{G}_{S}\right)\right)\right|$.

Teorema 7.10: Um grafo plano triangular estendido sem as arestas de construção possui uma representação retangular se e somente se podemos escolher vértices para adicionarmos arestas de construção de modo que não existe uma forma de separá-los utilizando um circuito de três arestas que não limita uma face.

Demonstração: Seja $\mathbb{G}$ um grafo plano triangular estendido que possui uma representação retangular. Suponha por absurdo que existe um circuito de três arestas $C$ em $\mathbb{G}$, de modo que $\mathbb{G}-V(C)$ divide o grafo nos subconjunto $V_{1}$ e $V_{2}$ e, sem perda de generalidade, todos vértices que possuem arestas de construção pertencem ao conjunto $V_{1}$. Como $v_{\infty}$ é adjacente a todos os vértices que possuem arestas de construção, ou $v_{\infty} \in V_{1}$ ou $v_{\infty} \in V(C)$. Seja $\mathbb{G}_{S}=\mathbb{G}\left[V_{1} \cup V(C)\right]$. A face criada em $\mathbb{G}_{S}$ é limitada por três arestas. Seja $E_{\text {constr }}^{\mathbb{G}_{S}}$ o conjunto das arestas de construção pertencente a $E\left(\mathbb{G}_{S}\right)$. Como $v_{\infty}$ e todos os vértices que possuem arestas de construção pertencem a $V\left(\mathbb{G}_{S}\right)$, então $\left|E_{\text {constr }}^{\mathbb{G}_{S}}\right|=4$. Aplicando o lema 7.7 temos que $\operatorname{Ex}\left(V\left(\mathbb{G}_{S}\right)\right)=\left|R I\left(V\left(\mathbb{G}_{S}\right)\right)\right|+1$. Um absurdo, pois pelo teorema 7.6, $\mathbb{G}$ não possui uma representação retangular.

Agora, vamos provar a suficiência. Seja $\mathbb{G}$ um grafo plano triangular estendido sem as arestas de construção. Vamos adicionar as arestas de construção a $\mathbb{G}$ de modo que $\mathbb{G}$ satisfaça as condições do lema 7.8. Seja $K=\left(V_{S} ; \overline{V_{S}}\right)$ um corte de $\mathbb{G}$ e $\mathbb{G}_{S}=\mathbb{G}\left[V_{S}\right]$. Vamos mostrar que $\operatorname{Ex}\left(V\left(\mathbb{G}_{S}\right)\right) \leq\left|R I\left(V\left(\mathbb{G}_{S}\right)\right)\right|$ e assim $\mathbb{G}$ satisfaz o teorema 7.6. Ademais, precisamos mostrar que $\mathbb{G}_{S}$ não possui um vértice $v$ de modo que $\mathbb{G}_{S}-v$ não é conexo e que $\mathbb{G}_{S}$ possui mais que dois vértices. Seja $C$ o circuito que define a face criada em $\mathbb{G}_{S}$. Considere os dois casos:

Caso 1: $|C| \geq 4$. Assim, $\mu_{S} \geq 4$. Deste modo, mesmo se $v_{\infty}$ e todos os vértices que possuem arestas de construção pertencerem a $V\left(\mathbb{G}_{S}\right)$, temos que pelo lema $7.7, \operatorname{Ex}\left(V\left(\mathbb{G}_{S}\right)\right) \leq$ $\left|R I\left(V\left(\mathbb{G}_{S}\right)\right)\right|$.

Caso 2: $|C|=3$. Pelo lema 7.7, temos: $\operatorname{Ex}\left(V\left(\mathbb{G}_{S}\right)\right)=\left|R I\left(V\left(\mathbb{G}_{S}\right)\right)\right|+2\left|E_{\text {constr }}^{\mathbb{G}_{S}}\right|+\left|E_{\text {constr }}^{K}\right|-7$. Se $\mid V\left(\mathbb{G}_{S}\right)=3$, então pelo menos uma das arestas de construção não pertence a $E\left(\mathbb{G}_{S}\right)$, pois $\mathbb{G}_{S}$ satisfaz o lema 7.8. Implicando que $\left|E_{\text {constr }}^{\mathbb{G}_{S}}\right|<4, \operatorname{logo} 2\left|E_{\text {constr }}^{\mathbb{G}_{S}}\right|+\left|E_{\text {constr }}^{K}\right| \leq 7$. Portanto, $\operatorname{Ex}\left(V\left(\mathbb{G}_{S}\right)\right) \leq\left|R I\left(V\left(\mathbb{G}_{S}\right)\right)\right|$. Se $C$ é um triângulo onde $\mathbb{G}_{S}-C$ possui mais que uma componente conexa, então, por hipótese, ao menos um vértice não pertence a $V\left(\mathbb{G}_{S}\right)$. Assim, $\left|E_{\text {constr }}^{\mathbb{G}_{S}}\right| \leq 3$. Portanto, $\operatorname{Ex}\left(V\left(\mathbb{G}_{S}\right)\right) \leq\left|R I\left(V\left(\mathbb{G}_{S}\right)\right)\right|$.

Portanto, pelo teorema 7.6, $\mathbb{G}$ possui uma representação retangular. 


\section{Parte II}

\section{Problema do Desenho Retangular}





\section{8}

\section{PROBLEMA DO DESENHO RETANGULAR}

Neste capítulo iremos apresentar o problema de desenho retangular e também que o mesmo é equivalente ao problema de representação retangular.

Um desenho retangular de um grafo plano $\mathbb{G}$, é um grafo isomorfo a $\mathbb{G}$ onde todas suas arestas são verticais ou horizontais e todas as faces são retangulares. Além disso, em um desenho retangular, as arestas que incidem na face externa formam um retângulo. A figura 8.1A representa um desenho retangular e a figura 8.1B representa um caso onde as arestas são orientadas na horizontal ou vertical, entretanto as arestas incidentes na face externa não formam um retângulo.

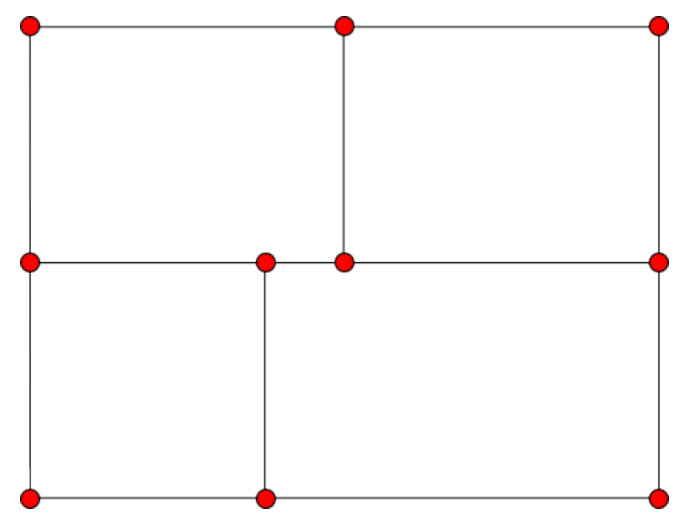

(A) Desenho retangular

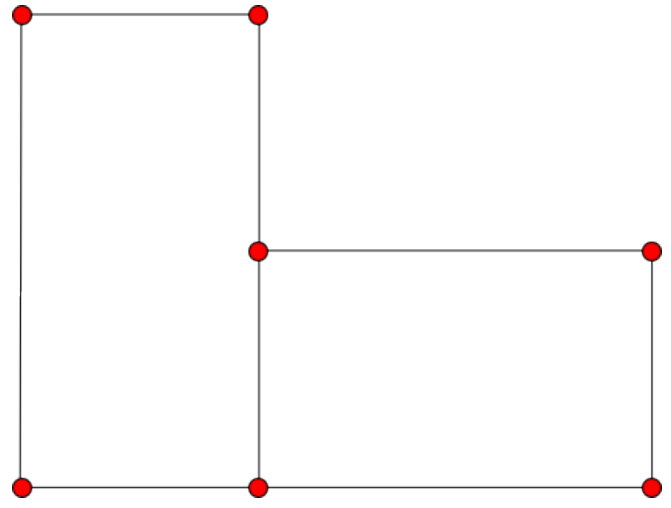

(B) Desenho não retangular

Figura 8.1: Desenho retangular e não retangular de um grafo planar 
A seguir, vamos enunciar problemas equivalentes aos problemas relacionados a representação retangular (problemas 1 e 2 enunciados no capítulo 3). Basicamente, estes problemas consistem em dado um grafo plano $\mathbb{G}$ de entrada verificar se existe um desenho retangular de $\mathbb{G}$.

Problema 3. Problema de Decis Ão do Desenho Retangular

Entrada: $\quad$ Grafo plano $\mathbb{G}=(V, E)$.

SAIDA: $\quad$ Decidir se existe um grafo isomorfo a $\mathbb{G}$ que possui todas suas arestas desenhadas na horizontal ou vertical e que as arestas que incidem na face externa formam um retângulo.

Problema 4. Problema da COnstruçÃo do Desenho Retangular

Entrada: $\quad$ Grafo plano $\mathbb{G}=(V, E)$.

SAIDA: Construir um grafo isomorfo a $\mathbb{G}$ que possui todas suas arestas desenhadas na horizontal ou vertical e que as arestas que incidem na face externa formam um retângulo.

Ilustramos na figura $8.2 \mathrm{~B}$ o grafo de saída para o problema 4 com o grafo de entrada apresentado na figura $8.2 \mathrm{~A}$.

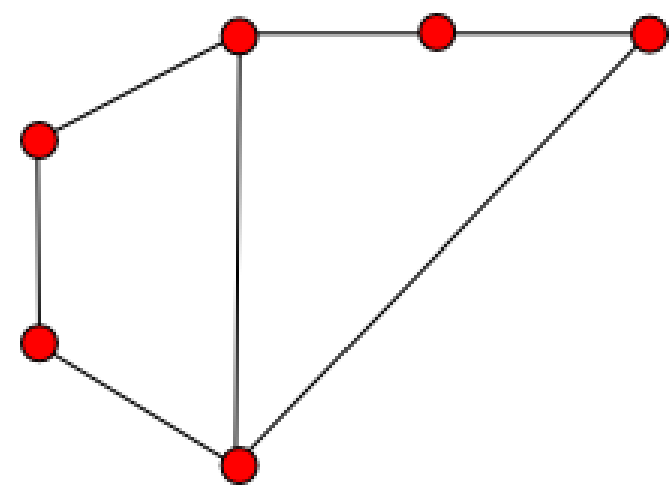

(A)

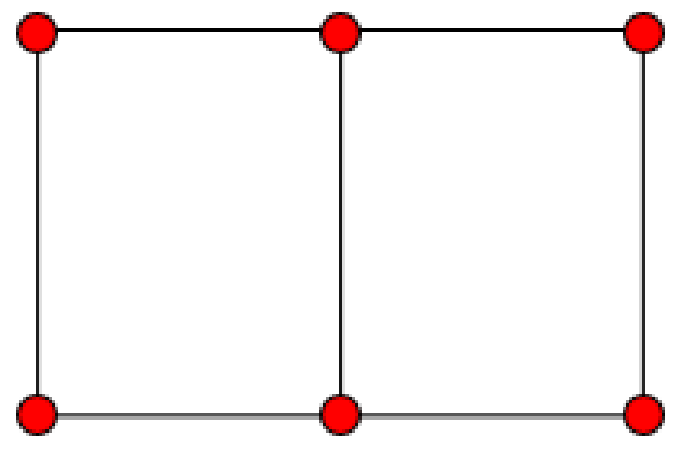

(B)

Figura 8.2: Ilustração da entrada e saída do problema de desenho retangular. (A) Grafo de Entrada. (B) Desenho retangular do grafo ilustrado em (A).

Para mostrar a equivalência entre os problemas vamos utilizar o conceito de dualidade de grafos. Este conceito foi previamente apresentado no Capítulo 2.

Lema 8.1: Os problemas 1 e 3 são equivalente.

Demonstração. Seja $\mathbb{G}$ um grafo que é entrada do problema 3. Lembre que supomos que $\mathbb{G}$ não possui nenhum vértice com grau maior que 3 , considere o grafo $\mathbb{G}^{*}$ como o grafo dual de $\mathbb{G}$. Seja $v_{\infty}$ o vértice de $\mathbb{G}^{*}$ que representa a face externa do grafo $\mathbb{G}$. Considere $\mathbb{T}=\mathbb{G}^{*}-v_{\infty}$. 
Se $|V(\mathbb{T})|<3$, então temos um grafo plano que é entrada do problema 1 e a solução deste problema também é solução do problema 3 .

Caso $|V(\mathbb{T})| \geq 3$, perceba que $\mathbb{T}$ é um grafo triangulado. Logo, $\mathbb{T}$ é um grafo de entrada do problema 1 e a solução deste problema também é solução do problema 3.

É fácil ver que também podemos reduzir o problema 1 para o problema 3 através de uma construção contrária à apresentada anteriormente. Portanto, os problemas 1 e 3 são equivalentes.

Lema 8.2: Os problemas 2 e 4 são equivalentes.

\subsection{REVISÃo BibliogRÁFICA}

No contexto de desenho retangular, o primeiro trabalho relevante foi publicado por Thomassen [Tho84] onde são apresentadas condições suficientes e necessárias para um grafo plano possuir um desenho retangular. A figura 8.3A ilustra um grafo que não possui desenho retangular e a figura 8.3B ilustra um que possui.

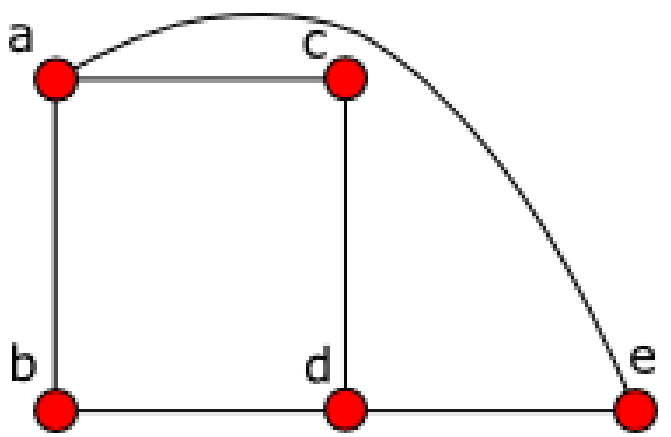

(A)

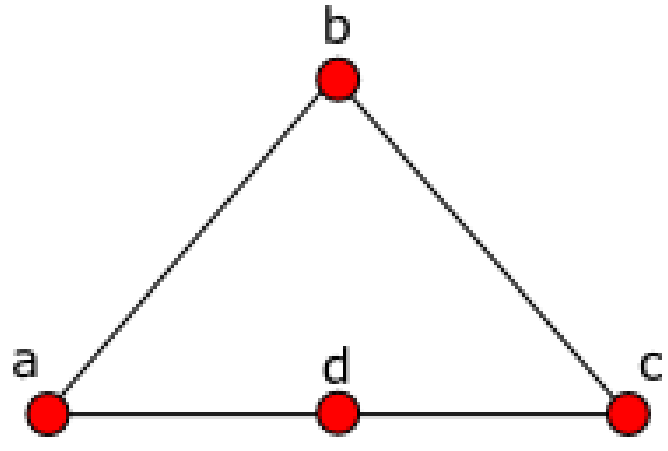

(B)

Figura 8.3: (A) Grafo plano que não possui desenho retangular. (B) Grafo plano que possui desenho retangular

A caracterização feita por Thomassen em [Tho84] implica em um algoritmo eficiente, que segundo Rahman et al. [RNN98], e uma implementação direta de seu método possui complexidade $O\left(n^{3}\right)$, onde $n$ é o número de vértices do grafo de entrada. Entretanto, o resultado de Thomassen serviu como base para outros trabalhos que apresentam algoritmos mais eficientes, como por exemplo, [RNN98, RNG04, He93], os quais apresentam complexidade linear no número de vértices do grafo de entrada. Um dos trabalhos que se destaca é o algoritmo utilizado por Rahman et al. em [RNN98] que utiliza, basicamente, conceitos de caminhos, ciclos e do algoritmo de busca em profundidade. 
Iremos apresentar no próximo capítulo a caracterização de Thomassen e a seguir o trabalho de Rahman et al.. 


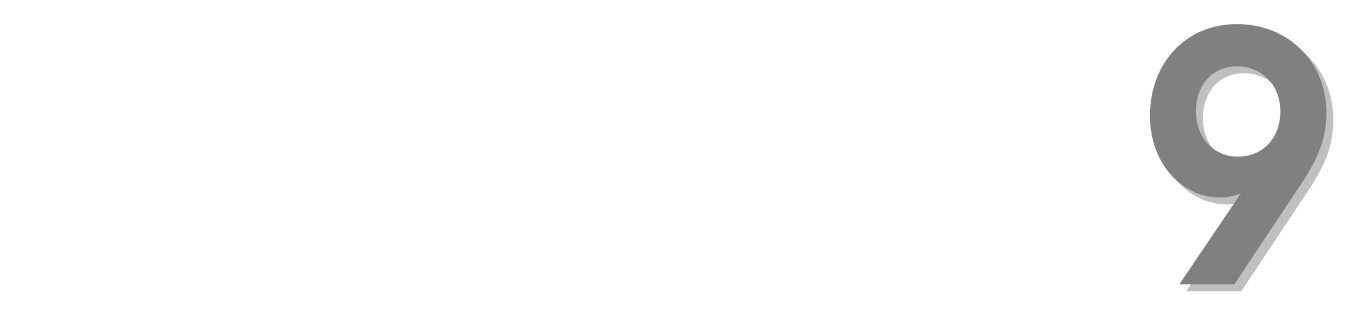

\section{CARACTERIZAÇÃo De THOMASSEN}

Como já discutido anteriormente, obter um algoritmo para solucionar este problema "equivale" a encontrar uma abordagem distinta para solucionar o problema da representação retangular.

Neste capítulo, iremos apresentar o trabalho de Thomassen [Tho84]. Seu trabalho é uma caracterização do problema de desenho retangular para uma classe de grafos planos.

\subsection{TRANSFORMAÇÃO DO GRAFO DA ENTRADA}

Pela definição do problema grafo retangular, é necessário que o grafo retangular possua ao menos 4 vértices (figura 9.1). Ainda, note que o grafo de entrada deve possuir exatamente 4 vértices de grau 2 enquanto todos os outros possuem grau 3 (figura 9.2).

Considere um grafo plano 3-conexo $\mathbb{H}$ de modo que todo vértice possui grau 3. Vamos modificar $\mathbb{H}$ adicionando quatro vértices de grau 2. Cada vértice será adicionado em uma aresta conforme a figura 9.3 ilustra.

Os 4 vértices de grau 2 irão representar os cantos de um desenho retangular de $\mathbb{H}$ (figura $9.4)$.

É fácil ver que não podemos dividir qualquer aresta da face externa de $\mathbb{H}$. Um fato 


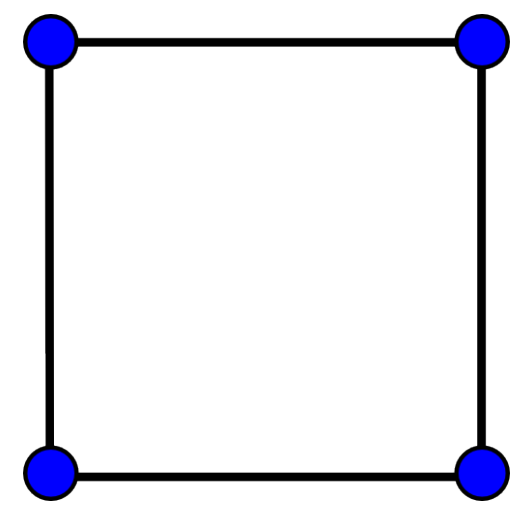

Figura 9.1: Caso trivial $(|V(\mathbb{G})|=4)$.

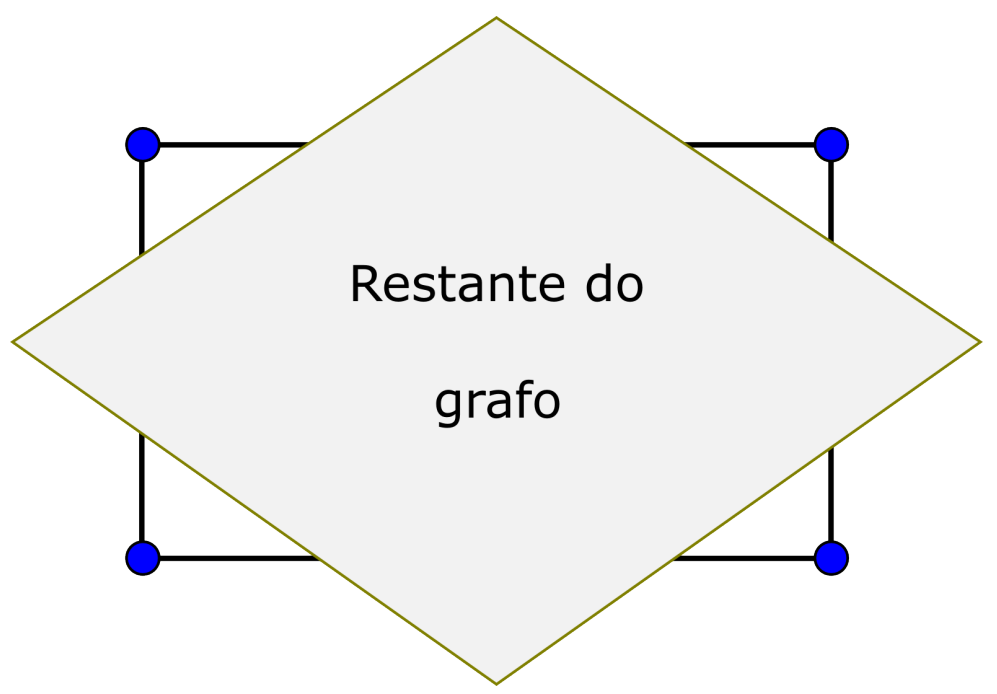

Figura 9.2: Necessidade da existência de 4 vértices de grau 2.

importante é que toda face interna de um grafo retangular possui 4 arestas, logo, só podemos realizar a divisão de, no máximo, 4 arestas da face externa que garantem que todas faces internas possuem pelo menos 4 arestas.

Considere que o grafo de entrada do problema é um grafo 2-conexo. Pois, já foram realizadas as subdivisões necessárias.

Antes de enunciar o teorema principal deste trabalho, vamos definir uma estrutura.

Seja $\mathbb{G}$ um grafo plano e $\mathbb{C}$ um circuito de $\mathbb{G}$. Dizemos que uma $\mathbb{C}$-componente é um subgrafo interno a $\mathbb{C}$ que é, ou um $K_{2}$, onde os vértices (não as arestas) do $K_{2}$ pertencem a $\mathbb{C}$, ou uma componente conexa $\mathbb{H}$ de $\mathbb{G}-V(\mathbb{C})$ juntamente com as arestas que conectam $\mathbb{H}$ a $\mathbb{C}$ e com os vértices incidentes em $\mathbb{H}$. Estes vértices que pertencem a $\mathbb{C}$ serão chamados de vértices de anexo. 

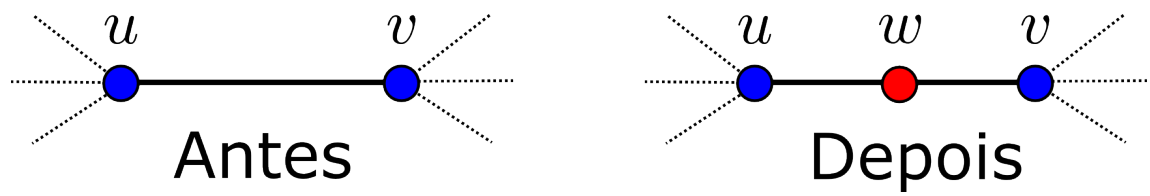

Figura 9.3: Subdivisão de uma aresta para adicionar o vértice $w$ que possui grau 2.

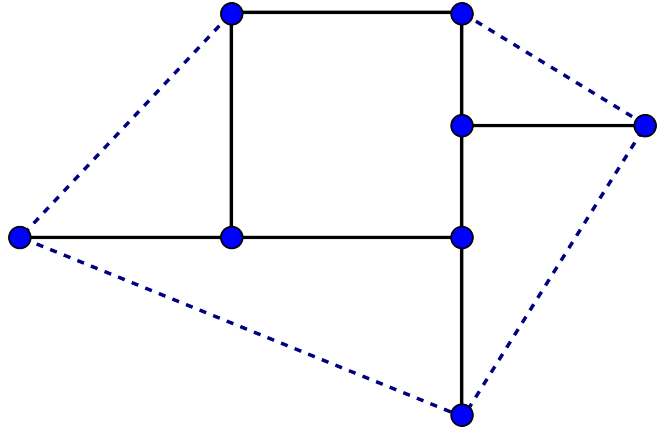

(A)

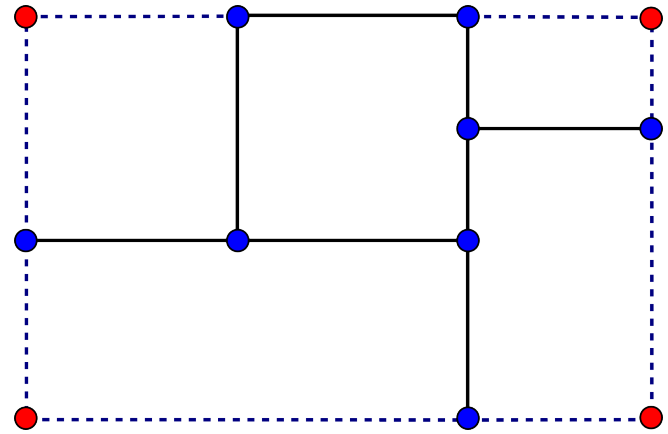

(B)

Figura 9.4: As arestas pontilhadas de (A) representam as arestas do grafo 3-conexo que serão divididas e criados vértices de grau 2, os quais são ilustrados em (B) (vértices vermelho).

\subsection{CaracterizAÇÃo DE Thomassen}

Teorema 9.1: Seja $\mathbb{G}$ um grafo plano 2-conexo de modo que 4 vértices do circuito da face externa $C_{0}$ possuem grau 2 e todos os outros vértices possuem grau 3.

Vamos particionar $C_{0}$ em quatro caminhos: $P_{O}, P_{N}, P_{L}$ e $P_{S}$, onde somente os extremos de cada caminho possuem grau 2.

Seja $\mathbb{G}^{\prime}$ um grafo retangular isomorfo ao grafo $\mathbb{G}$. $O$ circuito da face externa $C_{0}^{\prime}$ (que é um retângulo) é formado pelos caminhos $P_{i}$, onde $i=\{O, N, L, S\}\left(C_{0}^{\prime}=\right.$ $\left.P_{O} \cup P_{N} \cup P_{L} \cup P_{S}\right)$ de modo que todas as arestas de $P_{N}$ e $P_{S}$ são horizontais e todas as arestas de $P_{O}$ e $P_{L}$ são verticais.

Existe um grafo $\mathbb{G}^{\prime}$ com $C_{0}^{\prime}$ fixo que é desenho retangular de $\mathbb{G}$ se e somente se:

(i) Para qualquer vértice $v \in V(\mathbb{G})-V\left(C_{0}\right)$ e qualquer conjunto $X$ de no máximo 3 arestas, $\mathbb{G}-X$ possui um caminho de $v$ a $C_{0}$ a menos que $X$ seja o conjunto das arestas incidentes em $v$;

(ii) Cada $C_{0}$-componente de $\mathbb{G}$ "intercepta" ao menos dois caminhos de $P_{i}, i \in$ $\{O, N, L, S\}$

(iii) Para qualquer aresta $\alpha \notin E\left(C_{0}\right)$, cada $C_{0}$-componente de $\mathbb{G}-\alpha$ intercepta pelo menos dois caminhos $P_{i}, i \in\{O, N, L, S\}$.

Demonstração: É fácil ver que as condições (i), (ii) e (iii) são necessárias para que $\mathbb{G}^{\prime}$ seja um desenho retangular de $\mathbb{G}$. 
Vamos mostrar agora a suficiência. Na figura 9.5, exibimos as configurações proibidas que desejamos evitar.

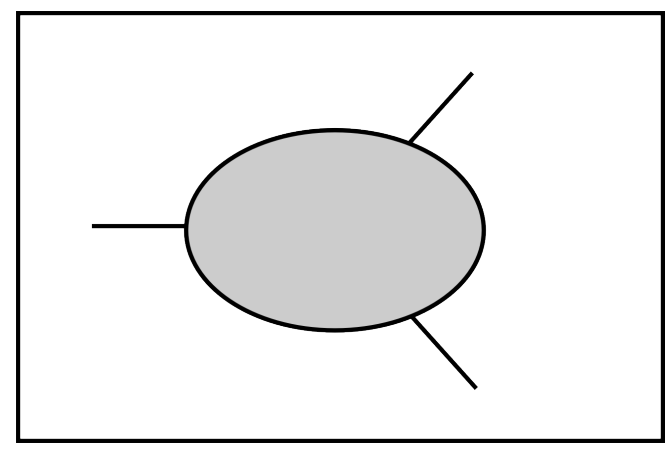

(A)

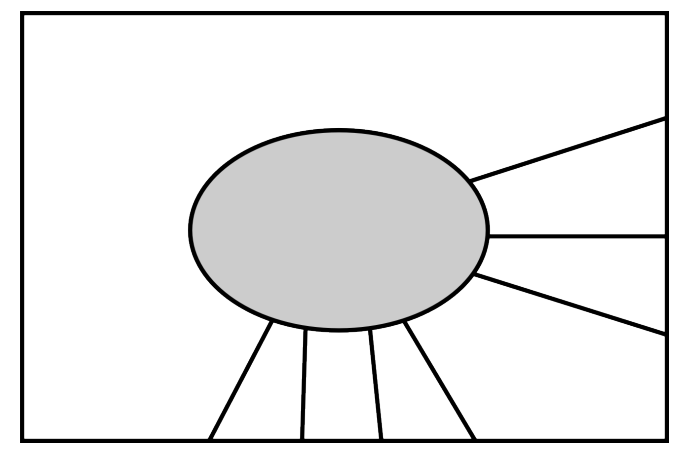

(B)

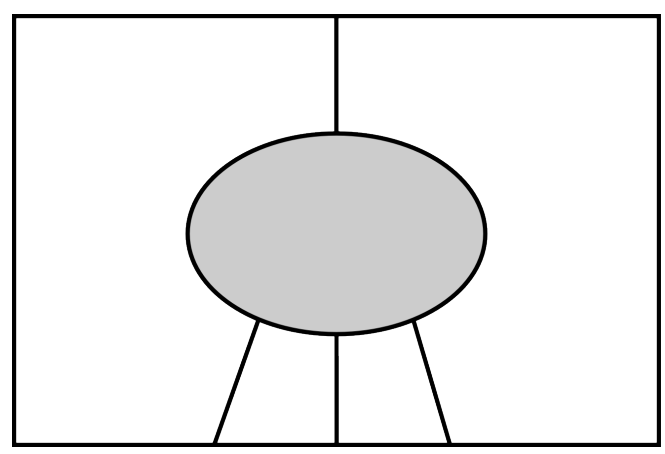

(C)

Figura 9.5: Configurações proibidas.

A prova a seguir será uma indução em $|E(\mathbb{G})|$.

Suponha que $\mathbb{G}$ possua uma $C_{0}$-componente $\mathbb{H}$ que incide, no máximo, em 3 caminhos $P_{i}, i \in\{O, N, L, S\}$.

Sem perda de generalidade, podemos assumir que $\mathbb{H}$ incide em $P_{N}, P_{S}$ e, possivelmente, em $P_{0}$. Note que $C_{0} \cup \mathbb{H}$ é 2-conexo. Seja $R$ um caminho de $\mathbb{H}$, de modo que $R$ mais o caminho $P_{L}$ e segmentos de $P_{N}$ e $P_{S}$ temos um circuito que descreve uma face de $\mathbb{G}$.

Para cada aresta de $R$, vamos definir que esta deve ser vertical em $\mathbb{G}^{\prime}$. Aplicamos a indução em cada um dos dois retângulos que foram formados. Claramente, os dois subgrafos de $\mathbb{G}$ satisfazem (i). Como $\mathbb{G}$ satisfaz (i), (ii) e (iii) e possui grau máximo 3, pela construção dos subgrafos de $\mathbb{G}$, temos que estes também satisfazem (ii) e (iii).

Suponha agora que $\mathbb{G}$ possua uma $C_{0}$-componente $\mathbb{H}$ que incide em todos os caminhos $P_{i}, i \in\{O, N, L, S\}$. Logo, $\mathbb{G}$ possui uma única $C_{0^{-}}$-componente.

Considere o caminho $R$ de $P_{N}$ a $P_{S}$, onde todos os vértices intermediários de $R$ pertencem a $V(\mathbb{G}) \backslash V\left(C_{0}\right)$.

Queremos escolher um caminho $R$ de modo que divida $\mathbb{G}$ em dois subgrafos para podermos aplicar a hipótese indutiva. Ademais, para aplicarmos a hipótese indutiva devemos definir como as arestas de $R$ serão em $\mathbb{G}^{\prime}$ : verticais ou horizontais. A seguir, vamos mostrar 
como encontrar $R$ e definir as orientações das arestas de $R$.

Agora, precisamos mostrar como escolher $R$. Note que o caminho $R$ irá gerar dois subgrafos, $\mathbb{G}_{1}$ e $\mathbb{G}_{2}$, de modo que $V\left(\mathbb{G}_{1}\right) \cap V\left(\mathbb{G}_{2}\right)=V(R)$ e que $V\left(\mathbb{G}_{1}\right) \cup V\left(\mathbb{G}_{2}\right)=V(\mathbb{G})$. Ainda, conforme a figura 9.6 ilustra, o circuito externo de cada subgrafo $\left(C_{0}^{1}\right.$ e $\left.C_{0}^{2}\right)$ é composto por segmentos dos caminhos $P_{N}$ e $P_{S}$, do caminho $R$ e de $P_{O}$ em $\mathbb{G}_{1}$ e de $P_{L}$ em $\mathbb{G}_{2}$.

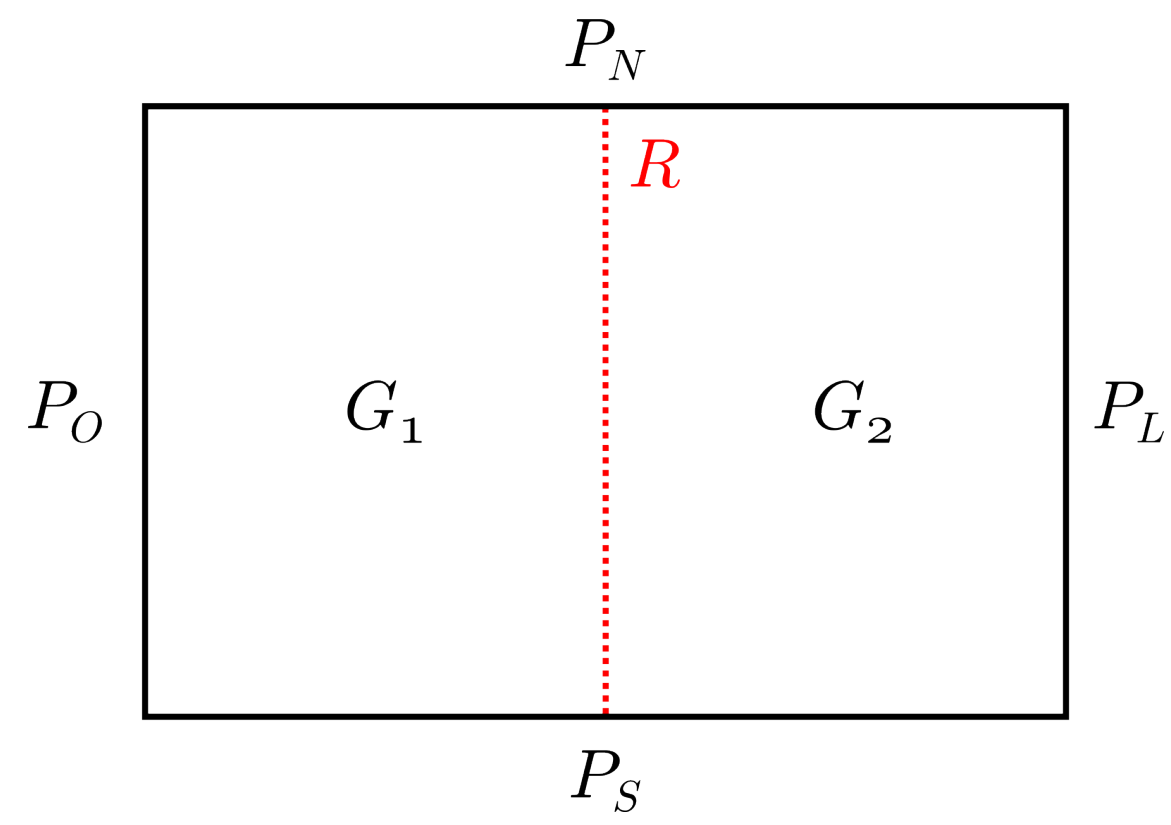

Figura 9.6: Subgrafos $\mathbb{G}_{1}$ e $\mathbb{G}_{2}$ gerados a partir de $R$.

Primeiramente, vamos mostrar que é possível escolher $R$ que induza $\mathbb{G}_{1}$ e $\mathbb{G}_{2}$ para aplicarmos a hipótese indutiva.

Se existe um $R$ que induz $\mathbb{G}_{1}$ e $\mathbb{G}_{2}$ que satisfazem (i), (ii) e (iii) não temos nada a provar.

Logo, vamos supor por absurdo, que para qualquer $R, \mathbb{G}_{1}$ ou $\mathbb{G}_{2}$ não satisfazem (i), (ii) ou (iii).

Perceba que para qualquer $R$, temos que $\mathbb{G}_{1}$ e $\mathbb{G}_{2}$ satisfazem (i). Vamos mostrar que é possível escolher um $R$ que também satisfaça (ii).

Suponha que $\mathbb{G}_{1}$ possui uma $C_{0}^{1}$-componente $H_{1}$ que não satisfaz (ii), logo, os vértices de anexo de $H_{1}$ pertencem a $R$ e a um dos caminhos $P_{N}$ ou $P_{S}$, digamos $P_{N}$.

Vamos definir como peso de $H_{1}$ como o tamanho do caminho mais curto em $R$ de um vértice de $P_{S}$ até um vértice de anexo de $H_{1}$.

Considere que vamos escolher um caminho $R$ de um vértice de $P_{N}$ a um vértice de $P_{S}$ que minimiza a soma dos pesos das $C_{0}^{1}$-componentes de $\mathbb{G}_{1}$ e $C_{0}^{2}$-componentes de $\mathbb{G}_{2}$ que violem (ii).

Se existir uma $C_{0}^{1}$-componente $H_{1}$ que viola (ii), então precisamos modificar $R$. Considere o caminho $R^{\prime}$ como sendo o menor caminho $R$ que conecta todos os vértices de anexo de $H_{1}$ em $R$ a um vértice de $P_{N}$. 
Vamos substituir $R^{\prime}$ por um caminho $S$, onde $S$ é um caminho que juntamente com $H_{1}$ e um segmento de $P_{N}$ forma um circuito externo a $H_{1}$ no qual não tem arestas de $H_{1}$. A figura 9.7 ilustra este procedimento. O segmento vermelho representa o caminho $R$, o segmento pontilhado é o caminho $R^{\prime}$. Os vértices vermelhos são os vértices de anexo de $H_{1}$.

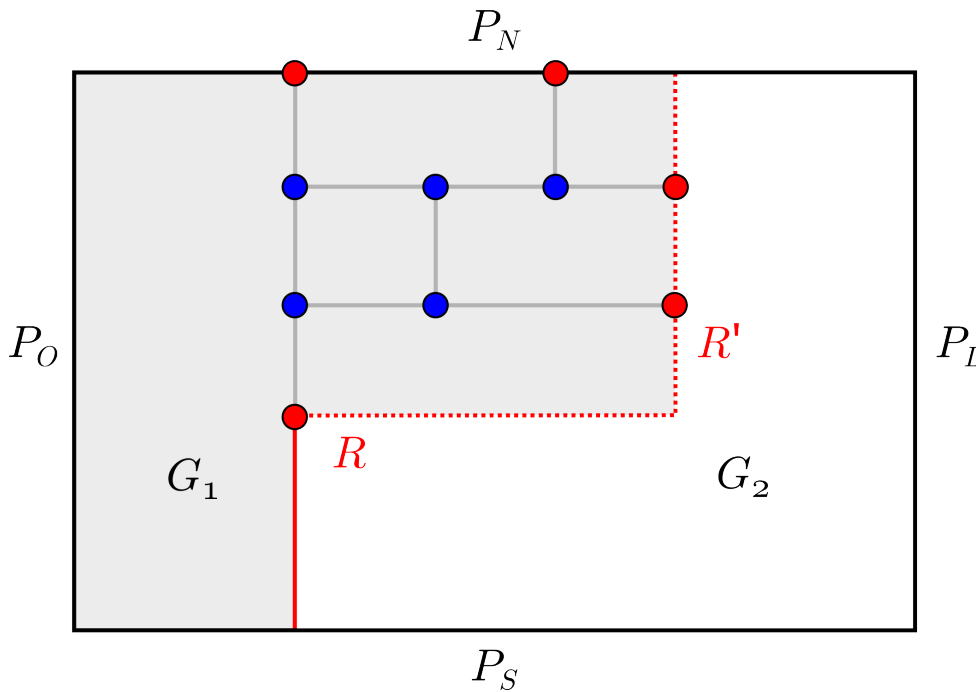

(A)

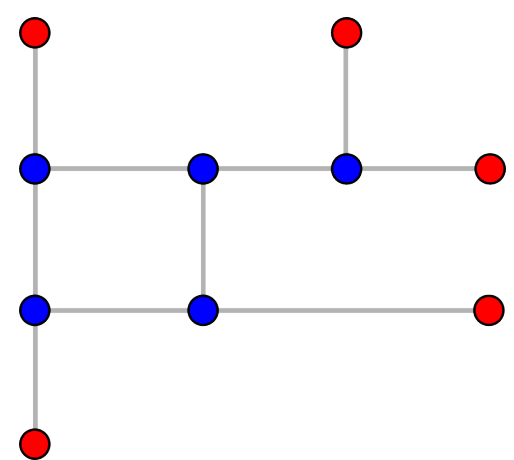

(B)

Figura 9.7: (A) $C_{0}^{1}$-componente juntamente com o caminho $R$ e $R^{\prime}$. (B) $C_{0}^{1}$-componente em destaque.

A escolha de $S$ e a troca por $R^{\prime}$ faz com que $H_{1}$ pertença ao subgrafo $\mathbb{G}_{2}$. Como $\mathbb{G}$ satisfaz (iii), então $H_{1}$ não viola (ii), logo, a soma dos pesos das $C_{0}^{1}$-componentes e $C_{0}^{2}$-componentes que violam (ii) diminui, um absurdo. Logo, existe um caminho $R$ de modo que $\mathbb{G}_{1}$ e $\mathbb{G}_{2}$ satisfazem (i) e (ii). (Figura 9.8)

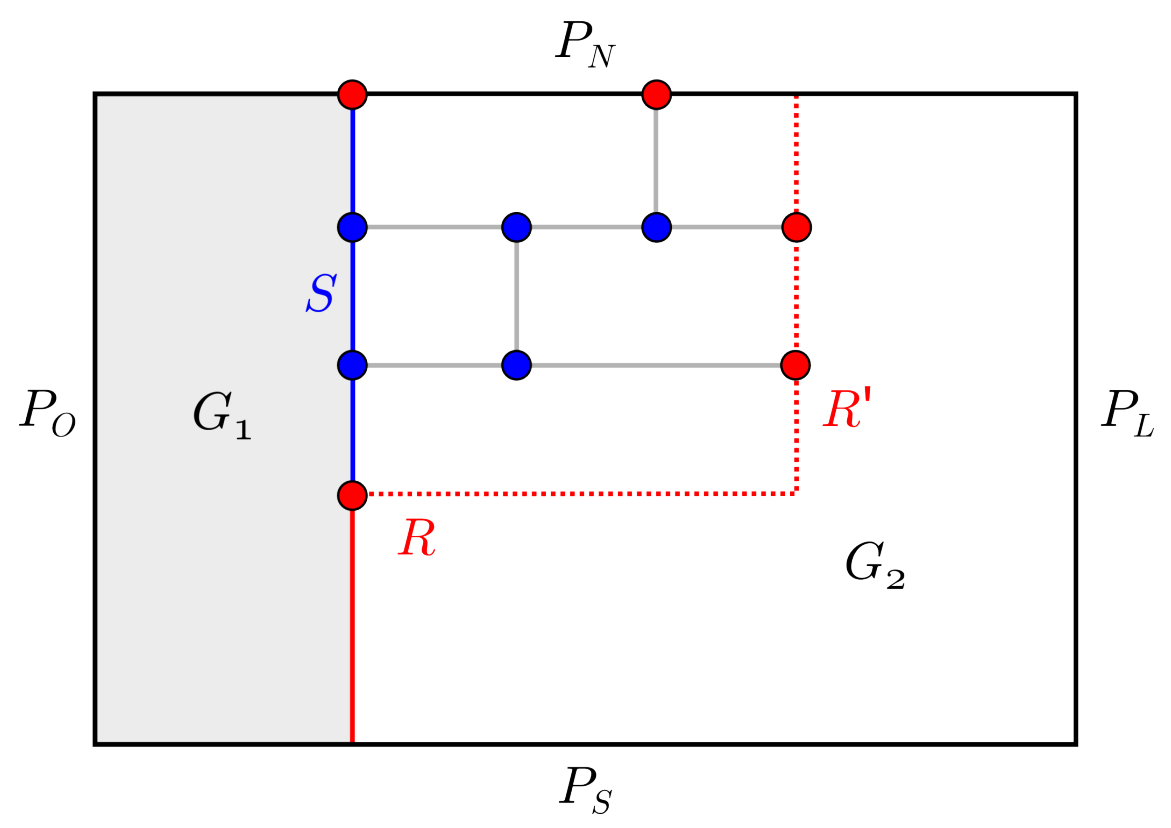

Figura 9.8: Subgrafos $\mathbb{G}_{1}$ e $\mathbb{G}_{2}$ gerados a partir da troca do caminho $R^{\prime}$ pelo caminho $S$ (caminho em azul).

Suponha então, que para todo $R$, temos que $\mathbb{G}_{1}$ ou $\mathbb{G}_{2}$ não satisfaz (iii). Sem perda de 
generalidade, suponha que $\mathbb{G}_{1}$ não satisfaz (iii). Então, existe uma aresta $\alpha$ tal que alguma $C_{0}^{1}$-componente $H_{1}$ de $\mathbb{G}_{1}-\alpha$ onde todos os vértices de anexo de $H_{1}$ em um dos seguintes caminhos: $P_{N}, P_{S}, P_{O}$ ou $R$. Entretanto, como $\mathbb{G}$ satisfaz (iii), então todos os vértices de anexo de $H_{1}$ pertencem a $R$. Ainda, como $H_{1}$ satisfaz (ii), $\alpha$ deve unir $H_{1}$ a $P_{O}$.

Seja $R^{\prime}$ o menor caminho de $R$ que contém todos os vértices de anexo de $H_{1}\left(V\left(H_{1}\right) \cap R\right)$. Definimos como peso de $H_{1}$, a cardinalidade de $E(R) \backslash E\left(R^{\prime}\right)$.

Assim, desejamos escolher um $R$ tal que $\mathbb{G}_{1}$ e $\mathbb{G}_{2}$ satisfazem (i) e (ii) e minimizem a soma dos pesos das $C_{0}^{1}$-componentes e $C_{0}^{2}$-componentes que violam (iii). Caso exista um $R$ que não viola (iii) (soma dos pesos igual a 0), então, aplicamos a hipótese indutiva em $\mathbb{G}_{1}$ e $\mathbb{G}_{2}$ e $\mathbb{G}^{\prime}$ é um grafo retangular isomorfo a $\mathbb{G}$. Caso contrário, então existe uma aresta $\alpha$ tal que, sem perda de generalidade, $\mathbb{G}_{1}-\alpha$ possui uma $C_{0}^{1}$-componente $H_{1}$ que viola a propriedade (iii).

Agora, vamos encontrar um caminho $S$ para substituir $R$. Mais precisamente, vamos utilizar $R$ para encontrar um caminho $S$ que contradiz a hipótese que a soma dos pesos é mínima.

Considere $R^{\prime}$ como sendo o menor caminho de $R$ que contém todos os vértices de anexo de $H_{1}\left(V\left(H_{1}\right) \cap R\right)$. Vamos substituir o segmento $R^{\prime}$ de $R$ pelo caminho $P$ que juntamente com $R^{\prime}$ forma um circuito externo a $H_{1}$. (Figura 9.9)

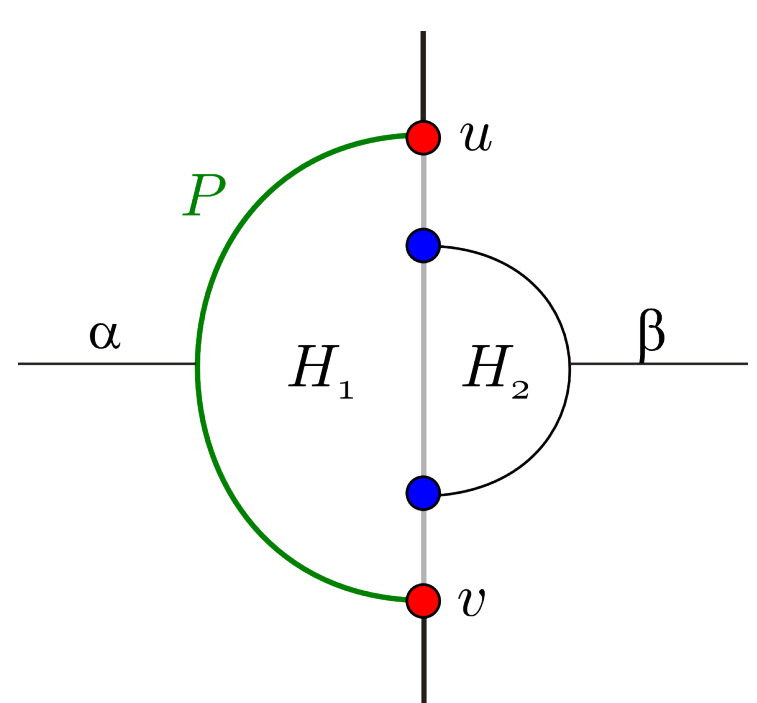

(A)

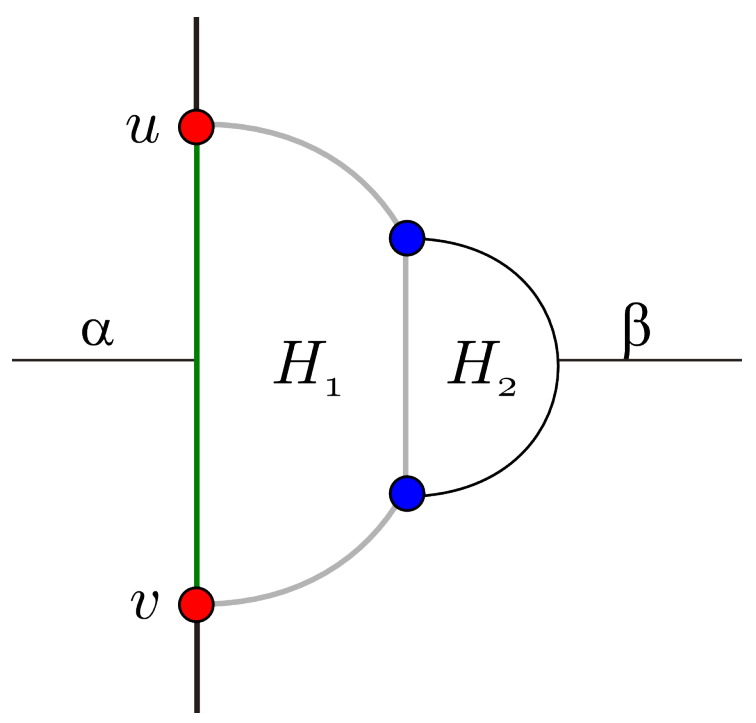

(B)

Figura 9.9: Ilustração do segmento $S$ obtido a partir de $R$.

Vamos mostrar que pela escolha de $R, \mathbb{G}_{2}$ possui uma aresta $\beta$ de modo que o grafo obtido a partir de $\mathbb{G}_{2}$ pela troca de $R$ por $S$ e remoção de $\beta$ possui uma $\left.\left[\left(C_{0}^{2} \backslash R\right)\right] \cup S\right]$-componente com o mesmo peso de $H_{1}$.

Considere $H_{2}$ como sendo uma $C_{0}^{2}$-componente, cujo todos os vértices de anexo pertencem ao caminho $R^{\prime}$. 
Como $\mathbb{G}$ satisfaz (ii), então existe ao menos uma aresta $\beta$ de $H_{2}$ que incide em algum vértice do caminho $P_{L}$. Caso $H_{2}$ seja um $K_{2}$, então $\beta$ é a única aresta de $K_{2}$, unindo um vértice de $R^{\prime}$ a um vértice de $P_{L}$. Precisamos mostrar agora, que $\beta$ é único, ou seja, caso $H_{2} \neq K_{2}$, então não existem duas arestas de $H_{2}$ que incidem no caminho $P_{L}$.

Suponha que existem ao menos duas arestas de $H_{2}$ que incidem em $P_{L}$. Logo, existiria um caminho distinto de $R$ (o atual caminho $S$ ) que minimiza a soma das componentes que violam a propriedade (iii). Pois, com a escolha de $S$, temos que $H_{1}$ não irá mais contribuir com a somatória, minimizando o total. Um absurdo, logo, só existe uma única aresta de $H_{2}$ que incide em $P_{L}$.

Ainda, conforme a figura 9.10 ilustra, existem, exatamente, 4 arestas incidindo no circuito externo aos subgrafos $H_{1}-\alpha$ e $H_{2}-\beta$ : $\gamma_{1}, \gamma_{2}, \alpha$ e $\beta$.

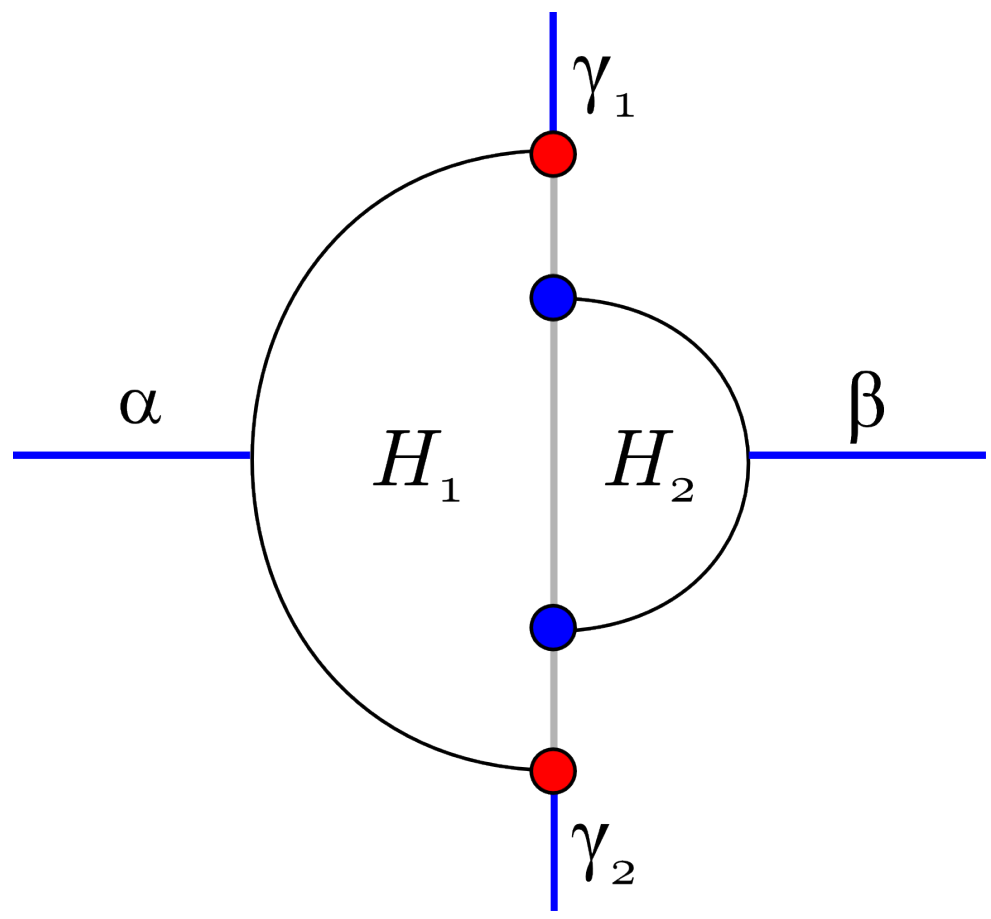

Figura 9.10: Arestas incidindo no circuito externo das componentes $\mathrm{H}_{1}$ e $\mathrm{H}_{2}$.

As arestas $\alpha$ e $\beta$ devem ser horizontais. A figura 9.11 ilustra como devemos desenhar em $\mathbb{G}^{\prime}$ as arestas do circuito externo a $H_{1}$ e $H_{2}, \alpha, \beta$ e parte do segmento do caminho $R$.

Precisamos garantir que as regiões 1, 2 e 3 satisfaçam as propriedades (i), (ii) e (iii).

É fácil ver que as regiões 1 e 2 são faces de $\mathbb{G}$. Como o circuito externo destas faces já possuem todas suas arestas definidas em $\mathbb{G}^{\prime}$ e satisfazem (i), (ii) e (iii). Logo, podemos aplicar a hipótese indutiva nestas faces.

Considere $\mathbb{G}_{3}$ como o grafo equivalente a região 3 . Precisamos garantir que $\mathbb{G}_{3}$ satisfaz (i), (ii) e (iii).

Como $\mathbb{G}$ satisfaz (i), $\mathbb{G}_{3}$ também satisfaz.

É fácil ver que a propriedade (ii) também é válida, pois se existir uma $C_{0}^{3}$-componente 

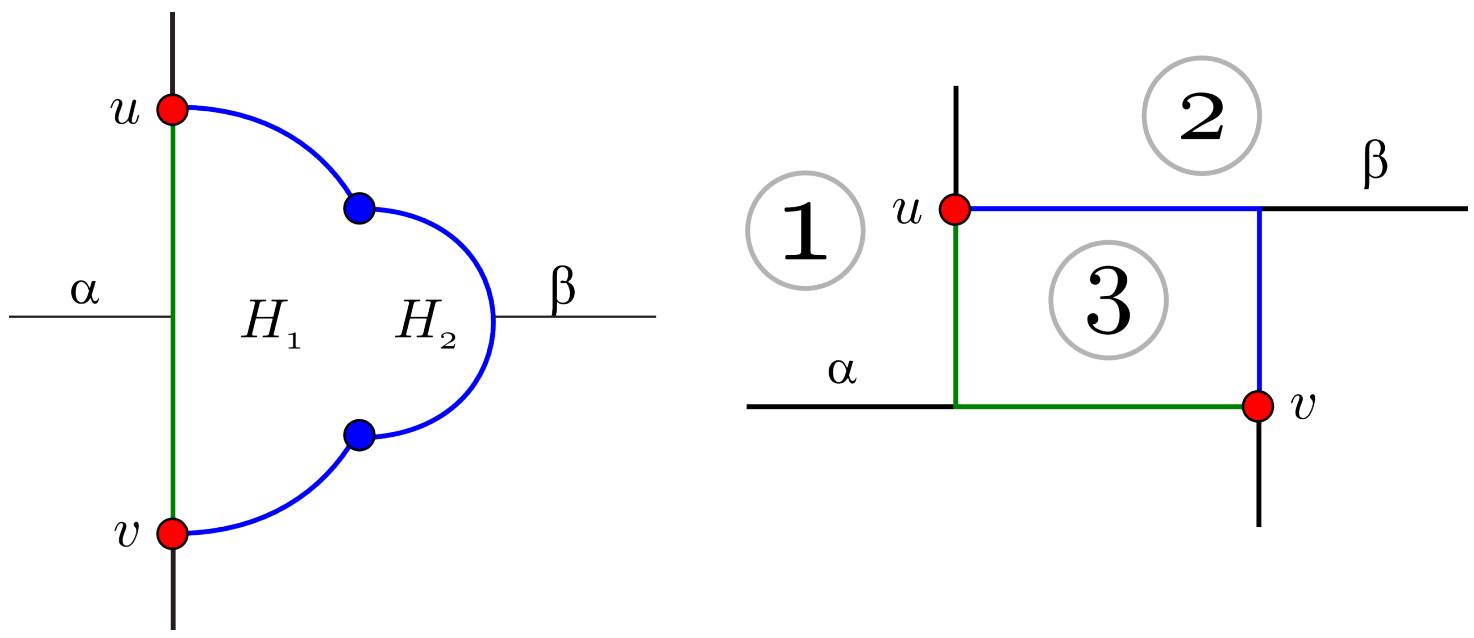

Figura 9.11: Ilustração da representação em $\mathbb{G}^{\prime}$ das arestas do circuito externo a $H_{1}$ e $H_{2}, \alpha, \beta$ e parte do segmento do caminho $R$.

(onde $C_{0}^{3}$ é o circuito da face externa de $\mathbb{G}_{3}$ ) que viola (ii), então esta componente contraria a escolha de $R$, já que escolhemos $R$ de modo que $\mathbb{G}_{1}$ e $\mathbb{G}_{2}$ satisfaziam (ii).

Agora, precisamos mostrar que (iii) também vale para $\mathbb{G}_{3}$. Como já argumentamos, o circuito externo a $H_{1}-\alpha$ e $H_{2}-\beta$ possui somente 4 arestas que saem: $\gamma_{1}, \gamma_{2}, \alpha$ e $\beta$. Logo, se existir uma $C_{0}^{3}$-componente em $\mathbb{G}_{3}$ que viola (iii), então $\mathbb{G}$ não satisfaz (i). Um absurdo, logo, podemos aplicar a hipótese indutiva em $\mathbb{G}_{3}$, e assim podemos desenhar $\mathbb{G}_{3}$ em $\mathbb{G}^{\prime}$.

Como a figura $9.12 \mathrm{~A}$ ilustra, pode existir mais do que uma $C_{0^{-}}^{1}$ componente ou $C_{0^{-}}^{2}$ componente que viola (iii). Vamos modificar $R$ conforme explicado anteriormente para cada componente. A figura 9.12B ilustra como os caminhos da figura 9.12A devem ser representados em $\mathbb{G}^{\prime}$, ou seja, se estes serão verticais ou horizontais.

Para concluir o passo indutivo, cada região da 9.12B satisfaz (i), (ii) e (iii), logo, devemos aplicar a hipótese indutiva na ordem que é indicado nesta figura.

Portanto, se o grafo $\mathbb{G}$ satisfaz as propriedades (i), (ii) e (iii), então é possível desenhar um grafo retangular $\mathbb{G}^{\prime}$ isomorfo a $\mathbb{G}$. 

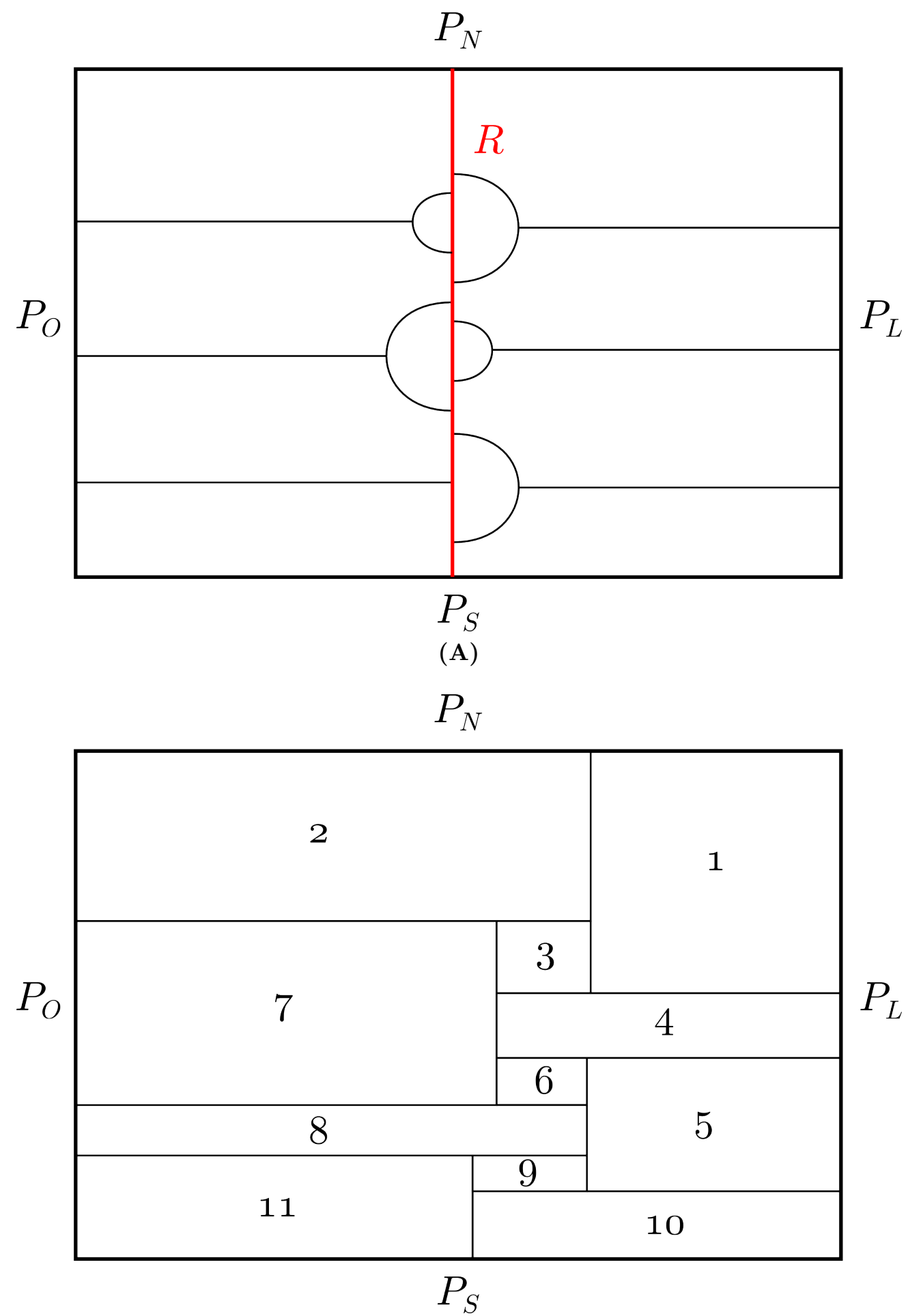

(B)

Figura 9.12: (A) Exemplo de um caminho $R$ juntamente com as componentes que violam a propriedade (iii). (B) Ilustração de como as componentes devem ser desenhadas em $\mathbb{G}^{\prime}$. 


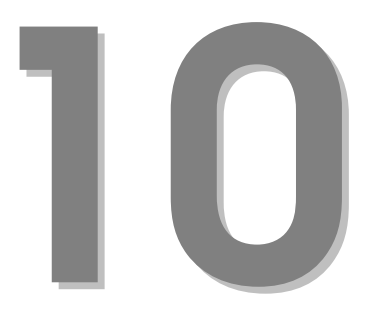

\section{AlgORITMO PARA O PROBLEMA DE DESENHO RETANGULAR BASEADO EM BUSCA EM PROFUNDIDADE}

Até o momento, apresentamos diversas abordagens para solucionar o problema da representação retangular e do desenho retangular. No contexto de desenho retangular, o trabalho desenvolvido por Rahman et al. [RNN98] se destaca por apresentar um algoritmo que utiliza programação dinâmica baseado na busca em profundidade que, além de possuir uma ideia relativamente simples, possui complexidade linear.

Outra motivação para estudarmos o algoritmo desenvolvido por Rahman et al. é que este utiliza a caracterização de Thomassen [Tho84] (capítulo 9) e a explora de uma forma distinta da apresentada anteriormente para criar seu algoritmo. Ainda, o trabalho de Thomassen desenvolve uma caracterização para o problema de decisão do desenho retangular, entretanto, Rahman et al. utiliza a caracterização para solucionar, além do problema de decisão, o problema de construção do desenho retangular. 


\subsection{VISÃO GERAL SOBRE O ALGORITMO}

Nesta seção, vamos considerar que estamos resolvendo os problemas relacionados ao desenho retangular de um grafo plano. Como já supomos anteriormente, o grafo $\mathbb{G}$ tem exatamente quatro vértices de grau 2 e todos os outros possuem grau 3. Também vamos assumir que sabemos qual é o ciclo mais externo de $\mathbb{G}$ e quais são os quatro cantos do grafo retangular (grafo resultante do desenho retangular de $\mathbb{G}$ ). Conforme já definido anteriormente, denominamos o ciclo externo de $\mathbb{G}$ como $C_{0}(\mathbb{G})$, ou somente $C_{0}$. $C_{0}$ é dividido em quatro caminhos $P_{N}, P_{S}, P_{O}$ e $P_{L}$, onde o primeiro e o último vértice de cada caminho possuem grau 2 e todos os outros vértices dos caminhos possuem grau 3.

O algoritmo é baseado na técnica de divisão e conquista. A ideia do algoritmo é encontrar um caminho $P=\left\langle p_{0}, p_{1}, \ldots, p_{n}\right\rangle$, onde nenhuma aresta de $P$ pertence a $E\left(C_{0}\right)$, de modo que $p_{0}$ e $p_{n}$ pertençam a caminhos opostos de $C_{0}$, digamos $P_{N}$ e $P_{S}$. Sem perda de generalidade, suponha que $p_{0}$ pertence a $P_{N}$ e $p_{n}$ pertence a $P_{S}$. Note que o vértice $p_{0}$ divide o caminho $P_{N}$ em dois. Assuma que $P_{N}^{E}$ é o segmento à esquerda de $p_{0}$ e $P_{N}^{D}$ é o segmento à direita de $p_{0}$ e que ambos possuem o vértice $p_{0}$. De modo análogo, o vértice $p_{n}$ define dois segmentos em $P_{S}: P_{S}^{E}$ e $P_{S}^{D}$.

É fácil ver que se podemos definir todas as arestas de $P$ como verticais, o caminho $P$ irá dividir $\mathbb{G}$ em dois subgrafos, digamos $G_{E}^{P}$ e $G_{D}^{P}$, onde $G_{E}^{P}$ é o subgrafo cujo ciclo externo $C_{0}\left(G_{E}^{P}\right)=P_{N}^{E} \cdot P \cdot P_{S}^{E} P_{O}$. De forma análoga, definimos $C_{0}\left(G_{D}^{P}\right)=P_{N}^{D} \cdot P \cdot P_{S}^{D} P_{L}$. A figura 10.1 ilustra esta divisão.

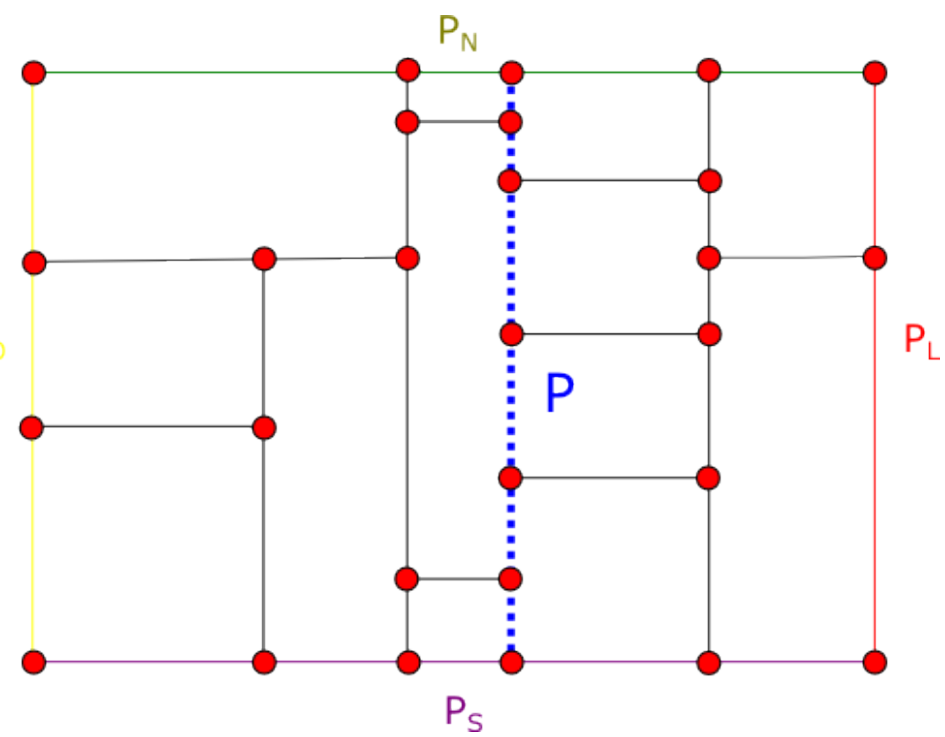

Figura 10.1: Exemplo de um grafo plano $\mathbb{G}$ que contém um caminho $P$ que divide $\mathbb{G}$ nos dois subgrafos: $G_{E}^{P}$ e $G_{D}^{P}$.

Entretanto, nem sempre é possível encontrar um caminho $P$ de modo que todas as suas arestas sejam verticais. Neste caso, o algoritmo escolhe um caminho $P$ e define, a partir 
de uma estratégia, se as arestas de $P$ serão verticais ou horizontais. Deste modo, também criamos dois subgrafos de $\mathbb{G}$, digamos $G_{E}^{P}$ e $G_{D}^{P}$. Estes subgrafos serão construídos de forma semelhante à apresentada anteriormente.

Por fim, tendo dividido o problema original em dois subproblemas independentes, a abordagem é aplicada a cada subproblema. Caso exista um desenho retangular para os subgrafos é fácil encontrar um desenho retangular para $\mathbb{G}$, e caso não exista, teremos um certificado que não existe um desenho retangular de $\mathbb{G}$.

Nas próximas seções iremos detalhar o algoritmo desenvolvido por Rahman et al. para encontrar um desenho retangular de um grafo plano que funciona, basicamente, seguindo as ideias apresentadas acima.

\subsection{CONCEITOS PRELIMINARES}

Nesta seção iremos apresentar os lemas em que o algoritmo é baseado. Os principais lemas são, fundamentalmente, partes do teorema 9.1 que já foi apresentado anteriormente neste trabalho. Os resultados que serão apresentados facilitam a construção do algoritmo.

Lema 10.1: Sejam $H_{1}, H_{2}, \ldots, H_{k}$ as $C_{0}$-componentes de um grafo plano $\mathbb{G}$. Considere $\mathbb{G}_{i}=C_{0}(\mathbb{G}) \cup H_{i}$, onde $1 \leq i \leq k$. $\mathbb{G}$ possui um desenho retangular com uma imersão retangular fixa de $C_{0}(\mathbb{G})$ se e somente se $G_{i}$ possui um desenho retangular com uma imersão retangular fixa de $C_{0}\left(G_{i}\right)=C_{0}(\mathbb{G})$ para cada $i, 1 \leq i \leq k$.

Demonstração: Observe que se $\mathbb{G}$ possui um desenho retangular, então as componentes foram desenhadas de modo a existir uma sobreposição entre elas. Ainda, note que dadas duas $C_{0}$-componentes, as arestas de uma destas sempre estará desenhada à esquerda de todas as arestas da outra componente. Agora, devemos mostrar que podemos desenhar o grafo $G_{i}$ para todo $i, 1 \leq i \leq k$.

É fácil ver que se $k=1$, a tese é verdadeira.

Assim, suponha que $k>1$ e que $H_{1}, H_{2}, \ldots, H_{k}$ está ordenado de modo que todas as arestas de $H_{i}$ estão à esquerda de todas as arestas de $H_{j}, i<j$.

Queremos mostrar que para cada grafo $G_{i}=C_{0}(\mathbb{G}) \cup H_{i}$, onde $1 \leq i \leq k$, existe uma forma de desenhar as outras $C_{0^{-}}$-componentes utilizando o desenho de $G_{i}$, para isso, precisamos mostrar que existem faces internas retangulares que contêm todas as arestas de uma determinada borda $\left(P_{O}\right.$ ou $\left.P_{L}\right)$ em cada grafo $G_{i}$. Vamos separar em três casos: $G_{1}$, $G_{i}$, onde $1<i<k$ e $G_{k}$. 
Caso $G_{1}$ : Como $H_{1}$ é a $C_{0}$-componente mais à esquerda, então neste caso, precisamos garantir que existe uma face interna $f$ de $G_{1}$ que contém todas as arestas de $P_{L}$ e $f$ é retangular.

Inicialmente, pela definição de $\mathbb{G}$, todas as faces de $H_{1}$ são retangulares. Logo, se existir uma face $f$ que satisfaça nossa afirmação, então $f$ é retangular. Suponha que não existe tal face. Assim, em $\mathbb{G}$, a $C_{0}$-componente $H_{1}$ possui uma aresta que incide em um vértice de $P_{L}$, uma contradição com o fato de $H_{1}$ estar à esquerda de todas as outras $C_{0^{-}}$ componentes de $\mathbb{G}$. Logo, existe uma face interna de $G_{1}$ que contém todas as arestas de $P_{L}$ e é retangular.

Caso $G_{i}, 1<i<k$ : Neste caso, $H_{i}$ é uma $C_{0}$-componente intermediária em $\mathbb{G}$, ou seja, para toda $C_{0}$-componente $H_{e}, \operatorname{com} e<i$ temos que $H_{e}$ está à esquerda de $H_{i}$ e toda $C_{0}$-componente $H_{d}$, com $i<d$ temos que $H_{i}$ está à esquerda de $H_{d}$. Logo, precisamos garantir que existem em $G_{i}$ duas face internas retangulares de modo que uma contém todas as arestas de $P_{O}$ e outra que contém todas as arestas de $P_{L}$.

Pelos mesmos argumentos do caso anterior, todas as faces internas são retangulares e que deve existir uma face interna retangular que contém todas as arestas de $P_{O}$ e uma face interna que contém todas as arestas de $P_{L}$, pois caso contrário $H_{i}$ não seria uma $C_{0}$-componente intermediária de $\mathbb{G}$.

Caso $G_{k}$ : Este último caso é análogo ao primeiro caso $\left(G_{1}\right)$. Assim, é fácil ver que existe uma face interna de $G_{k}$ que é retangular e possui todas as arestas de $P_{O}$.

Portanto, se existe um desenho retangular de $\mathbb{G}$, então deve existir para cada $G_{i}$ um desenho retangular com uma imersão retangular fixa de $C_{0}\left(G_{i}\right)=C_{0}(\mathbb{G})$ para cada $i$, $1 \leq i \leq k$.

Note que para mostrar a suficiência, a construção é similar à da necessidade. Logo, o lema é verdadeiro.

A partir de agora podemos assumir que $\mathbb{G}$ possui exatamente uma única $C_{0}$-componente. Os próximos lemas irão caracterizar as configurações proibidas para que o grafo $\mathbb{G}$ possua um desenho retangular.

Lema 10.2: $O$ grafo plano $\mathbb{G}$ não possui um desenho retangular se existe uma $C_{0}$-componente que possui um circuito com menos que quatro pernas.

Demonstração: Se um circuito em uma $C_{0}$-componente possui menos que quatro pernas, então é impossível encontrar um desenho de $\mathbb{G}$ onde todas as faces são retângulos. O problema são as faces externas ao circuito e que possuem aresta do circuito. Como o circuito possui menos que quatro pernas, ao menos uma destas faces não será retangular. 
A figura 10.2 ilustra o lema 10.2 quando o circuito possui três pernas. É fácil ver que esta também ilustra os casos com menos pernas.

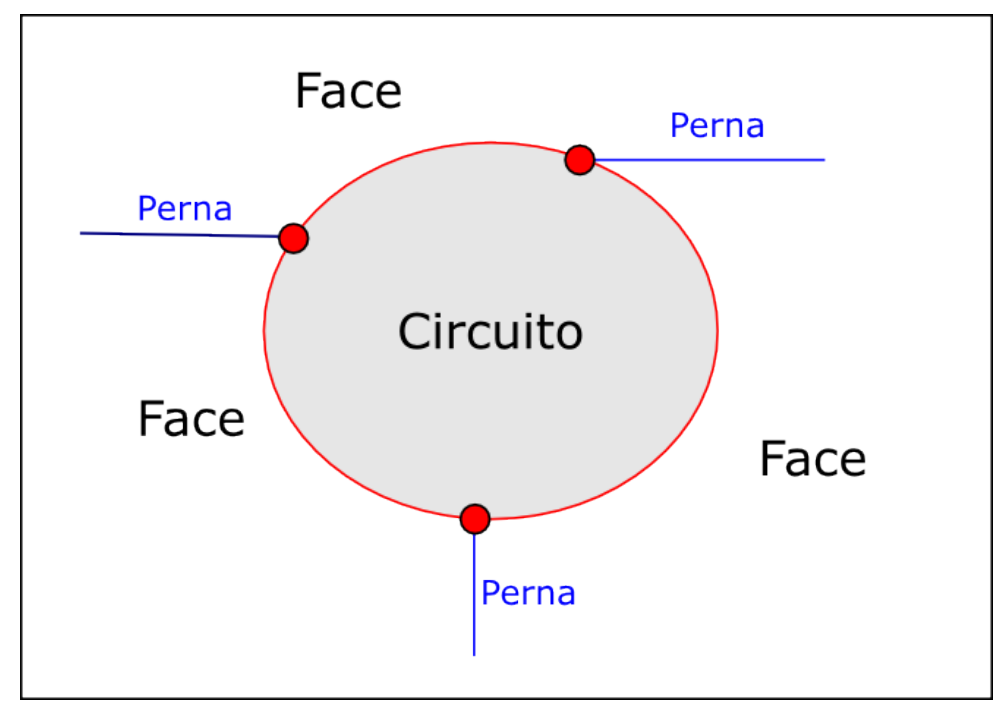

Figura 10.2: Ilustração do lema 10.2 [RNN98].

Um circuito $C$ é anexado a um caminho $P$ se $(i) P$ não contém algum vértice que está estritamente interno a $C$ e $(i i)$ a interseção de $C$ e $P$ é um único caminho que está contido em $P$. Seja $R=\left\langle v_{i n i}, \ldots, v_{\text {fim }}\right\rangle$ o caminho resultante da interseção entre $C$ e $P$. Chamamos o vértice $v_{i n i}$ de vértice de início de $C$ em relação a $P$ e o vértice $v_{f i m}$ de vértice do fim de $C$ em relação a $P$. Como estamos trabalhando com grafos planares, faz sentido falar da orientação dos caminhos. Assim, quando utilizarmos $Q_{h}(C)$, estamos nos referindo ao percurso em $C$ em sentido horário de $v_{i n i}$ até $v_{f i m}$. De modo análogo, $Q_{a h}(C)$ para o percurso em $C$ em sentido anti-horário de $v_{i n i}$ até $v_{f i m}$. Uma perna de $C$ é definida como horária em relação a $P$, se esta é incidente em um vértice de $V\left(Q_{h}(C)\right)-\left\{v_{i n i}, v_{f i m}\right\}$. O número de pernas horárias de $C$ em relação a $P$ é definido como $n_{h}(C)$. De modo análogo, definimos como $n_{a h}$ o número de pernas anti-horárias de $C$ em relação a $P$.

Define-se um circuito $C$ anexado a $P$ de circuito horário se $Q_{a h}(C)$ está contido em $P$ e de circuito anti-horário se $Q_{h}(C)$ está contido em $P$.

Dizemos que um circuito $C$ anexado a $P$ é um circuito crítico se $C$ é um circuito horário e $n_{h}(C) \leq 1$ ou $C$ é um circuito anti-horário e $n_{a h}(C) \leq 1$. A figura 10.3 ilustra um circuito horário que é crítico. 


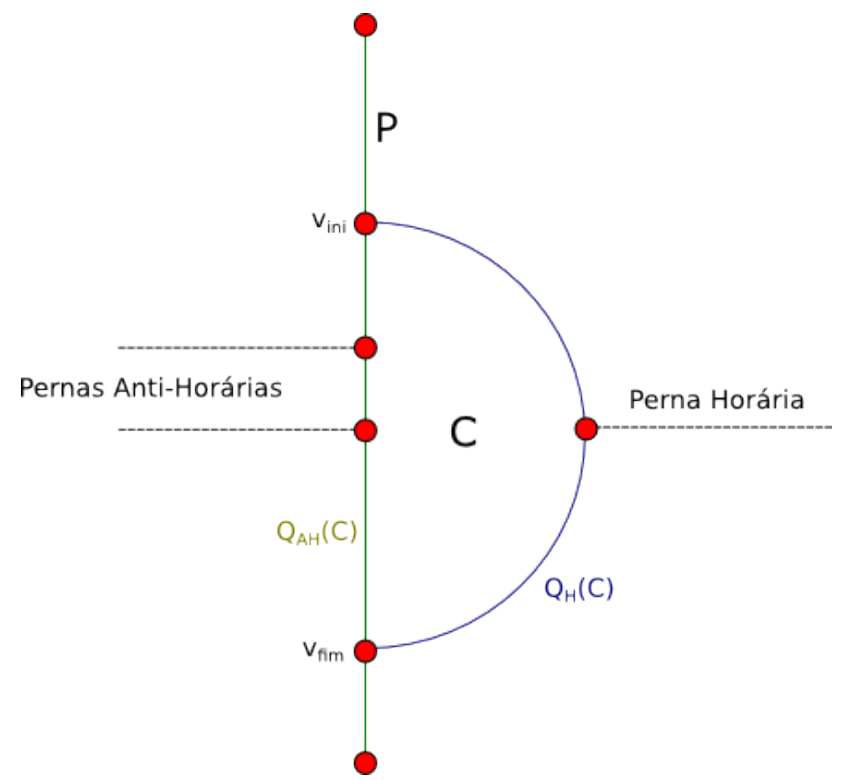

Figura 10.3: Circuito horário crítico $C$ anexado ao caminho P[RNN98].

Lema 10.3: $O$ grafo plano $\mathbb{G}$ não possui um desenho retangular se $\mathbb{G}$ possui um circuito crítico $C$ anexado a um dos caminhos $P_{N}, P_{S}, P_{O}$ ou $P_{L}$, exceto o ciclo externo $C_{0}$.

Demonstração: Considere que existe um circuito crítico $C$ em $\mathbb{G}$. Sem perda de generalidade, suponha que o circuito está anexado a $P_{N}$ e que este circuito é anti-horário. Logo, $n_{a h}(C) \leq 1$. Observe os casos na figura 10.4. Note que não é possível desenhar $C$ como um retângulo, pois em ambos os casos, as faces que possuem arestas de $C$ e estão no lado externo não serão retangulares.

Lema 10.4: $O$ grafo plano $\mathbb{G}$ não possui um desenho retangular se $\mathbb{G}$ possui um circuito crítico anti-horário $C \neq C_{0}$ anexado a um dos caminhos $P_{N} \cdot P_{O}, P_{L} \cdot P_{S}$, $P_{S} \cdot P_{O}$, ou $P_{O} \cdot P_{N}$, onde $n_{a h}(C)=0$.

Demonstração: A prova deste lema é análoga à do lema 10.3. Perceba que se existe um circuito crítico anti-horário $C \operatorname{com} n_{a h}(C)=0$, então existe somente uma face externa a $C$, logo, qualquer desenho retangular de $C$ impossibilita da face externa a $C$ ser retangular.

Os lemas 10.2, 10.3 e 10.4 caracterizam as $C_{0^{-}}$-componente que são proibidas. Uma $C_{0^{-}}$ componentes que satisfaz qualquer um destes lemas será chamada de componente ruim. Vamos provar mais adiante que um grafo plano $\mathbb{G}$ possui um desenho retangular se e somente não existem componentes ruins em $\mathbb{G}$. 


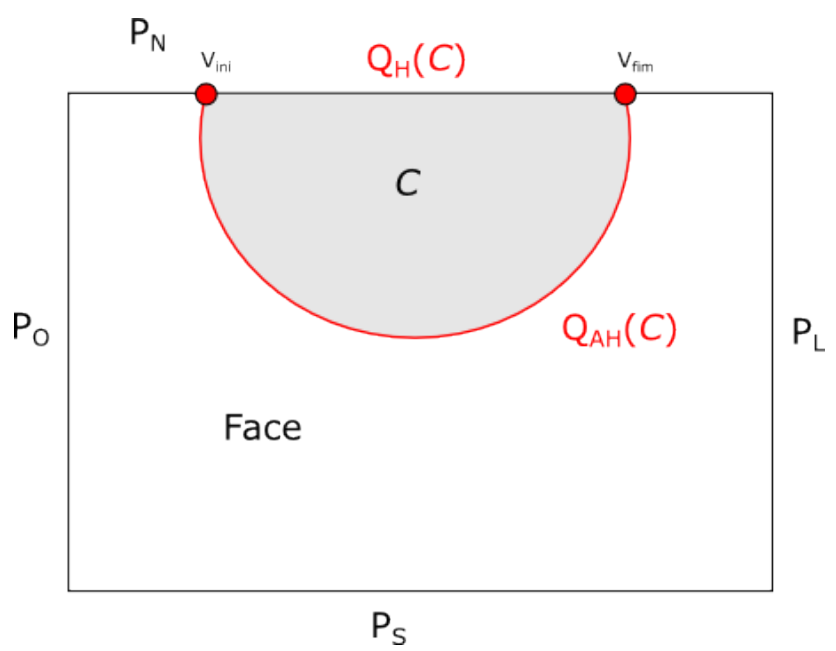

(A) $n_{a h}(C)=0$

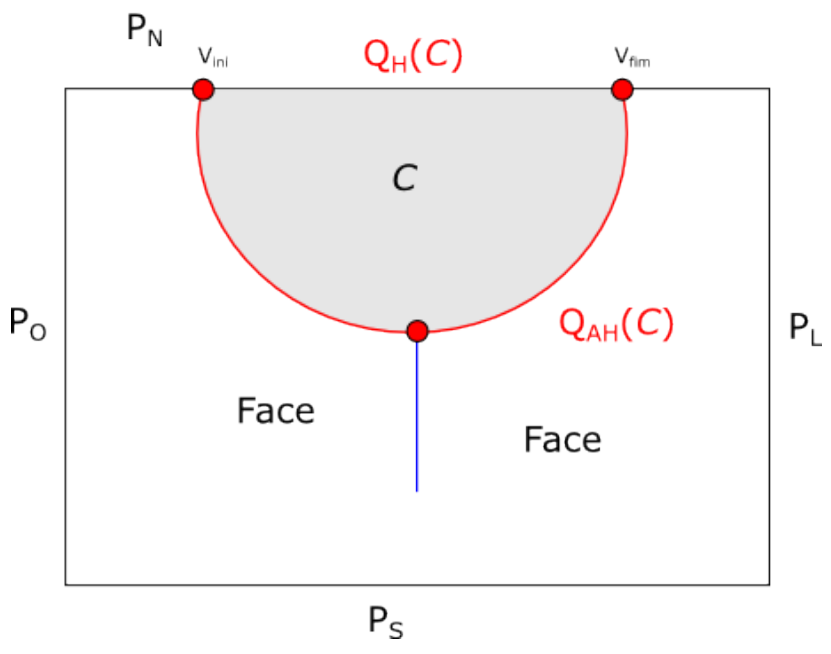

(B) $n_{a h}(C)=1$

Figura 10.4: Ilustração do lema 10.3 [RNN98]

Apresentamos até o momento características que mostram quais propriedades todos os grafos que possuem um desenho retangular devem possuir. A seguir, vamos enunciar resultados de modo que se o grafo $\mathbb{G}$ satisfazê-los, então existirá um desenho retangular de $\mathbb{G}$.

Considere que $P_{N}=\left\langle v_{0}, v_{1}, v_{2}, \ldots, v_{p}\right\rangle$ e $P_{S}=\left\langle u_{0}, u_{1}, u_{2}, \ldots, u_{q}\right\rangle$. Um NS-caminho é um caminho de um vértice $v_{i}$ de $P_{N}$ até um vértice $u_{j}$ de $P_{S}$ de modo que não contenha nenhum outro vértice de $C_{0}$. Como já apresentado anteriormente, podemos usar um NS-caminho $P$ para dividir $\mathbb{G}$ em dois subgrafos, $G_{E}^{P}$ e $G_{D}^{P}$. Se fixarmos $C_{0}\left(G_{E}^{P}\right)$ e $C_{0}\left(G_{D}^{P}\right)$ como retângulos, dizemos que o caminho $P$ é um $N S$-caminho particionador se não existe uma componente ruim em qualquer um dos grafos $G_{E}^{P}$ e $G_{D}^{P}$. Definimos $S N$-, LO- e $O L$-caminho particionador de modo análogo ao NS-caminho particionador. 
Como já vimos anteriormente, se encontrarmos algum caminho particionador, podemos dividir o grafo $\mathbb{G}$ e obter recursivamente um desenho retangular de $\mathbb{G}$.

Uma face interna de $\mathbb{G}$ é chamada de face da borda se existe alguma aresta da face que pertence a $C_{0}$. Dado uma face da borda $f$, o caminho da borda é o caminho que contém somente as arestas da borda de $f$ que conectam dois vértices de $C_{0}$ sem utilizar nenhuma aresta de $C_{0}$. Para $X, Y \in\{N, S, L, O\}$, um $X Y$-caminho da borda é um caminho da borda iniciado em um vértice do caminho $P_{X}$ e terminado em um vértice no caminho $P_{Y}$.

Quando for necessário interpretar um caminho da borda como um caminho orientado, considere que o caminho é o segmento das arestas de uma face da borda que foi percorrido no sentido horário.

Lema 10.5: Qualquer $N S$-, $S N-$, LO- ou $O L$-caminho da borda $P$ de $\mathbb{G}$ é um caminho particionador.

Demonstração: Seja $P$ um caminho da borda de $\mathbb{G}$. Sem perda de generalidade, vamos supor que $P$ é um $N S$-caminho da borda e que a face que contém $P$ também contém o caminho $P_{O}$. Assim, $P$ divide $\mathbb{G}$ em dois subgrafos: $G_{E}^{P}$ e $G_{D}^{P}$. (Figura 10.5)

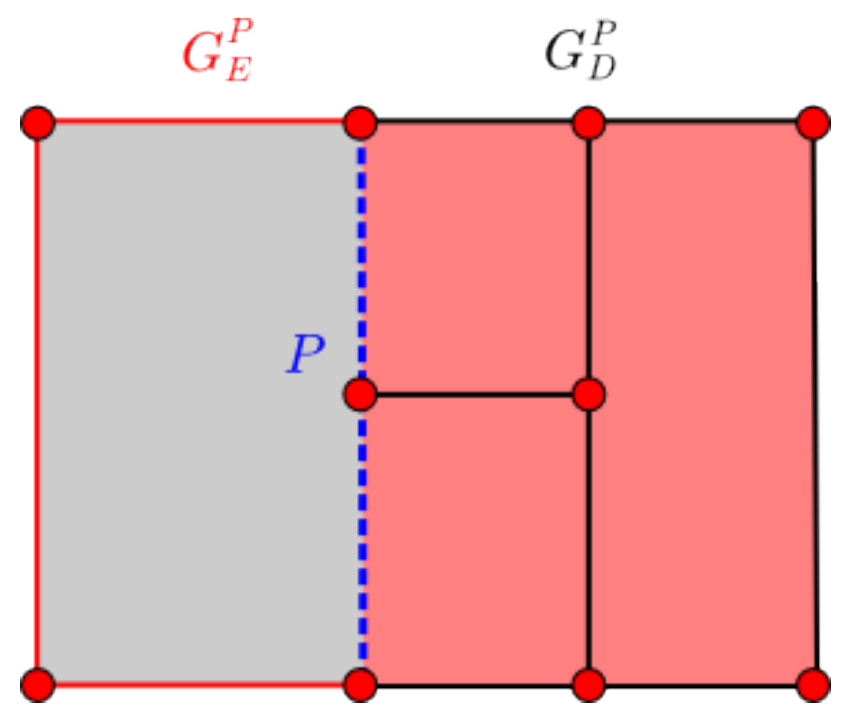

Figura 10.5: Ilustração de um NS-caminho da borda P. A esquerda ilustramos $G_{E}^{P}$ e a direita $G_{D}^{P}$.

Precisamos mostrar que $G_{E}^{P}$ e $G_{D}^{P}$ não possuem componentes ruins.

Pela suposição que $P$ é um $N S$-caminho da borda e que a face que o contém também possui o caminho $P_{O}$, então $G_{E}^{P}$ é somente um circuito, logo não existe uma componente ruim em $G_{E}^{P}$. Se $G_{D}^{P}$ também for um circuito, então a tese segue. Então, suponha que $G_{D}^{P}$ não é um circuito.

Suponha que existe uma componente ruim em $G_{D}^{P}$ conforme o lema 10.2, então existe uma componente ruim em $\mathbb{G}$, uma contradição. 
Suponha que existe uma componente ruim em $G_{D}^{P}$ conforme o lema 10.3 , então $G_{D}^{P}$ contém um circuito crítico anexado a $P$. Logo, $\mathbb{G}$ possui uma componente ruim conforme o lema 10.2, uma contradição.

Suponha que exista um circuito $C$ em $G_{D}^{P}$ conforme o lema 10.4. Então, ou $\mathbb{G}$ também possui o circuito $C$ de modo que satisfaz o lema 10.4, ou $\mathbb{G}$ possui uma componente ruim conforme o lema 10.3. Temos em ambos os casos uma contradição.

Portanto, $P$ é um caminho particionador.

Deste modo, pelo lema 10.5, se existe um caminho da borda, não temos mais nada a fazer. Vamos supor agora, que não existem tais caminhos em $\mathbb{G}$. Na ausência destes caminhos, vamos construir dois caminhos, $P_{H}$ e $P_{A H}$ que deverão particionar o grafo $\mathbb{G}$ em dois ou mais subgrafos que não possuem componentes ruins de modo a obtermos, recursivamente, um desenho retangular de $\mathbb{G}$. Como $P_{H} \neq P_{A H}$, então $E\left(P_{H}\right) \oplus E\left(P_{A H}\right)=E\left(P_{H}\right) \cup E\left(P_{A H}\right) \backslash$ $E\left(P_{H}\right) \cap E\left(P_{A H}\right)$ induz um conjunto de circuitos distintos nos vértices $C_{1}, C_{2}, \ldots, C_{k}, k \geq 1$, como ilustrado na figura 10.6 .

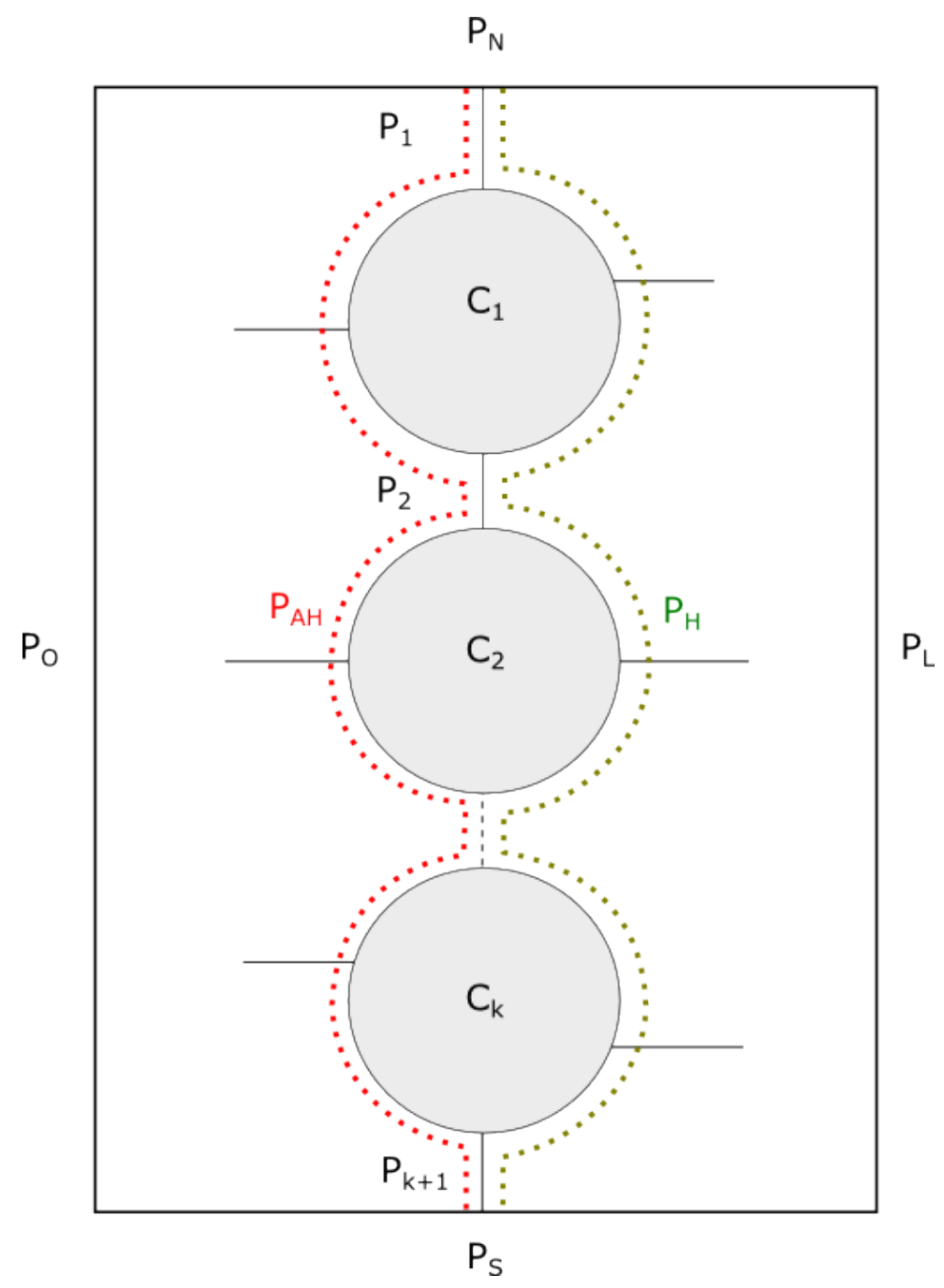

Figura 10.6: Circuitos induzidos por $P_{H}$ e $P_{A H}\left(P_{H} \oplus P_{A H}\right)$ [RNN98]. 
Logo, $P_{H}$ e $P_{A H}$ compartilham $k+1$ caminhos maximais $P_{1}, P_{2}, \ldots, P_{k+1}$. A escolha de $P_{H}$ e $P_{A H}$ será da seguinte forma, gostaríamos que o caminho $P_{H}$ percorresse os circuitos $C_{1}, C_{2}, \ldots, C_{k}$ no sentido horário e o caminho $P_{A H}$ no sentido anti-horário. Ainda, vamos escolher $P_{H}$ e $P_{A H}$ de modo que todo circuito $C_{i}$ possui, exatamente, quatro pernas. Em ordem horária, a primeira perna deve pertencer ao caminho maximal $P_{i}$, a segunda é uma perna horária, a terceira pertence ao caminho maximal $P_{i+1}$ e a quarta é uma perna antihorária. Antes de prosseguimos, precisamos apresentar uma definição.

Seja $\mathbb{G}$ um grafo plano e $C$ um circuito de $\mathbb{G}$. Definimos como interior de $C$ o subgrafo plano interno a $C$ unido com o circuito $C$. Para denotarmos este subgrafo utilizaremos a notação $\mathbb{G}_{\text {int }}(C)$, onde $\mathbb{G}$ é o nome do grafo e $C$ é o circuito em questão. Dizemos que uma aresta é uma perna de $C$, se esta incide em um vértice de $C$ e está localizada do lado externo de $C$.

Assim, podemos considerar que os caminhos $P_{H}$ e $P_{A H}$ dividem o grafo $\mathbb{G}$ nos seguintes subgrafos: $\mathbb{G}_{E}^{P_{A H}}, \mathbb{G}_{D}^{P_{H}}, \mathbb{G}_{\text {int }}\left(C_{1}\right), \mathbb{G}_{\text {int }}\left(C_{2}\right), \ldots, \mathbb{G}_{\text {int }}\left(C_{k}\right)$.

Assim, temos o seguinte lema.

Lema 10.6: Assuma que não existe uma componente ruim em $\mathbb{G}$ e que um ciclo $C$ em uma $C_{0}$-componente possui exatamente quatro pernas dividindo $C$ em quatro caminhos: $P_{N}^{\prime}, P_{S}^{\prime}, P_{L}^{\prime}$ e $P_{O}^{\prime}$. Se considerarmos $\mathbb{H}$ como sendo o subgrafo induzido pelo interior de $C$, então o subgrafo $\mathbb{H}$ não possui uma componente ruim para qualquer desenho retangular de $C$, cuja imersão planar é definido pelos caminhos $P_{N}^{\prime}, P_{S}^{\prime}, P_{L}^{\prime}$ e $P_{O}^{\prime}$.

Demonstração: Dado um circuito $C$ possuindo uma imersão planar definida pelos caminhos $P_{N}^{\prime}, P_{S}^{\prime}, P_{L}^{\prime}$ e $P_{O}^{\prime}$, é fácil ver que se existe uma componente ruim em $\mathbb{H}$, então existe uma componente ruim em $\mathbb{G}$. Uma contradição com o fato que $\mathbb{G}$ não possui uma componente ruim.

Pelo lema 10.6, podemos assumir que não existe um componente ruim em nenhum dos grafos $\mathbb{G}_{\text {int }}\left(C_{1}\right), \mathbb{G}_{\text {int }}\left(C_{2}\right), \ldots, \mathbb{G}_{\text {int }}\left(C_{k}\right)$ para nenhuma imersão retangular de $C_{1}, C_{2}, \ldots, C_{k}$. Para cada circuito $C_{i}$, onde $1 \leq i \leq k$, temos duas possibilidades de imersões retangulares, ambas ilustradas na figura 10.7. Deste modo, existem $2^{k}$ configurações possíveis de imersão planar dos circuitos definidos por $P_{H}$ e $P_{A H}$, implicando que no desenho retangular, as arestas de $P_{H}$ e $P_{A H}$ serão horizontais e verticais. A figura 10.8 ilustra uma das possíveis imersões planares de $P_{H}, P_{A H}$ e dos circuitos $C_{1}, C_{2}, \ldots, C_{k}$.

Como já vimos anteriormente, nenhum dos grafos $\mathbb{G}_{\text {int }}\left(C_{1}\right), \mathbb{G}_{i n t}\left(C_{2}\right), \ldots, \mathbb{G}_{i n t}\left(C_{k}\right)$ possui uma componente ruim. Logo, precisamos analisar dois grafos, o à esquerda de $P_{A H}\left(G_{E}^{P_{A H}}\right)$ e o grafo à direita de $P_{H}\left(G_{D}^{P_{H}}\right)$. Entretanto, é fácil ver que o ciclo mais externo não possui 

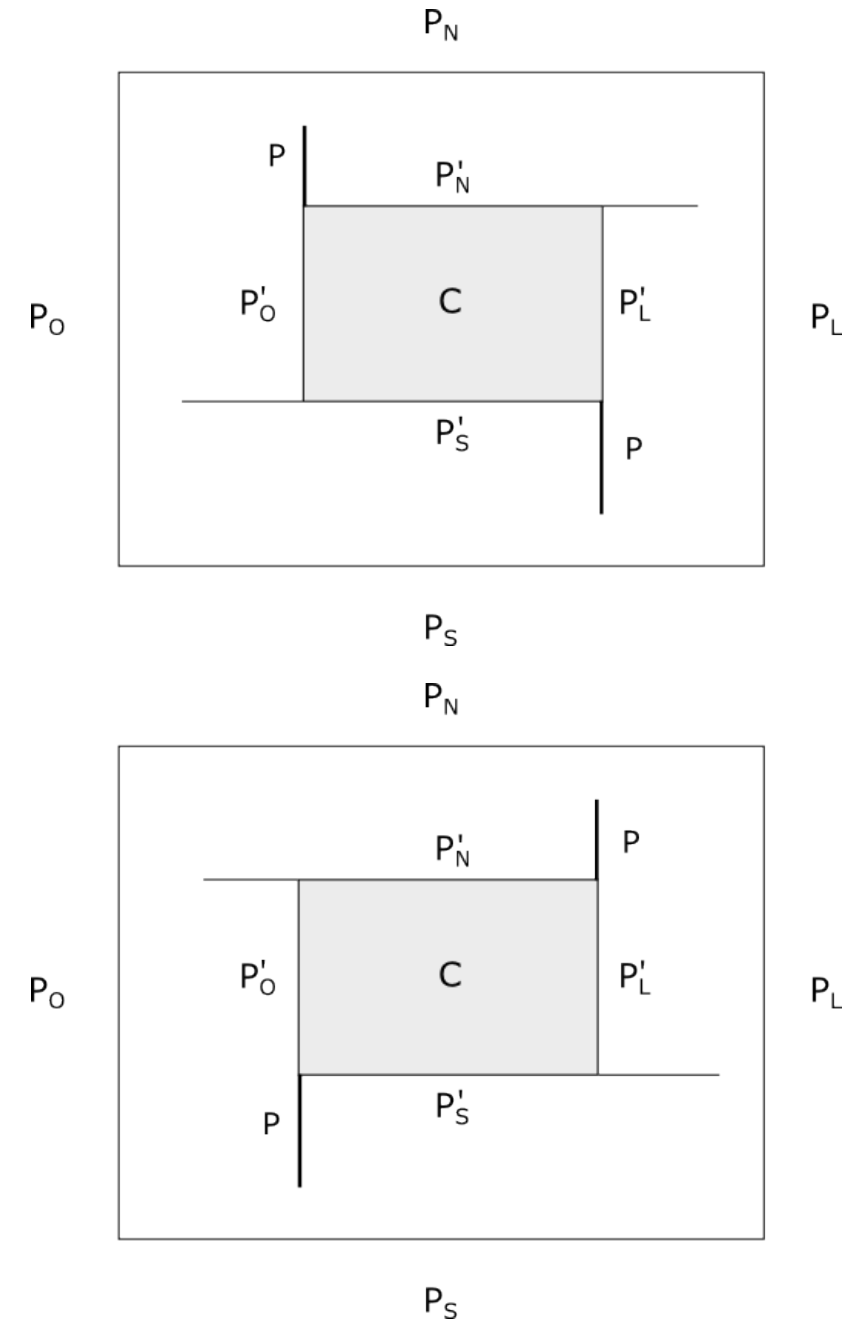

Figura 10.7: Possibilidades de imersão de um circuito $C$ [RNN98].

uma imersão retangular.

Seja $G_{1}$ um grafo obtido de $G_{E}^{P_{A H}}$ contraindo todas as arestas de $P_{A H}$ que são horizontais, ou seja, todas as arestas que pertencem aos lados horizontais da imersão planar dos circuitos $C_{1}, C_{2}, \ldots, C_{k}$. Considere como $P_{A H}^{\prime}$ o caminho resultante da operação de contração das arestas de $P_{A H}$. Note que a imersão do circuito externo do grafo $G_{1}$ é retangular, pois $P_{A H}^{\prime}$ é um segmento vertical. Se não existir um desenho retangular em $G_{1}$ então, não existe tal desenho em $\mathbb{G}$. Caso exista, precisamos mostrar como estender este desenho retangular para $\mathbb{G}$. Antes de mostrar como estender o desenho retangular de $G_{1}$ devemos observar alguns fatos importantes. Seja $P^{\prime}$ o segmento de $P_{A H}$ que também pertence a um circuito $C_{i}$, para qualquer $1 \leq i \leq k$. Primeiramente, sabemos que só existe um perna anti-horária em $C_{i}$ em relação a $P_{A H}$, seja $v$ o vértice de $P_{A H}^{\prime}$ que esta perna incide. Note que $v$ é canto do retângulo definido pelo circuito externo de $C_{i}$. Ainda, como as arestas de $P_{A H}^{\prime}$ definem dois lados do retângulo de $C_{i}$, então todas as arestas antes de $v$ possuem uma orientação, digamos horizontal, e todas as arestas depois de $v$ possuem uma orientação contrária, digamos vertical. A implicação mais importante destes fatos é que em $G_{1}$ nenhuma aresta incide em $V\left(P_{A H}^{\prime}\right) \backslash\{v\}$. Pois se existir uma aresta incidente, contrariamos o fato que 


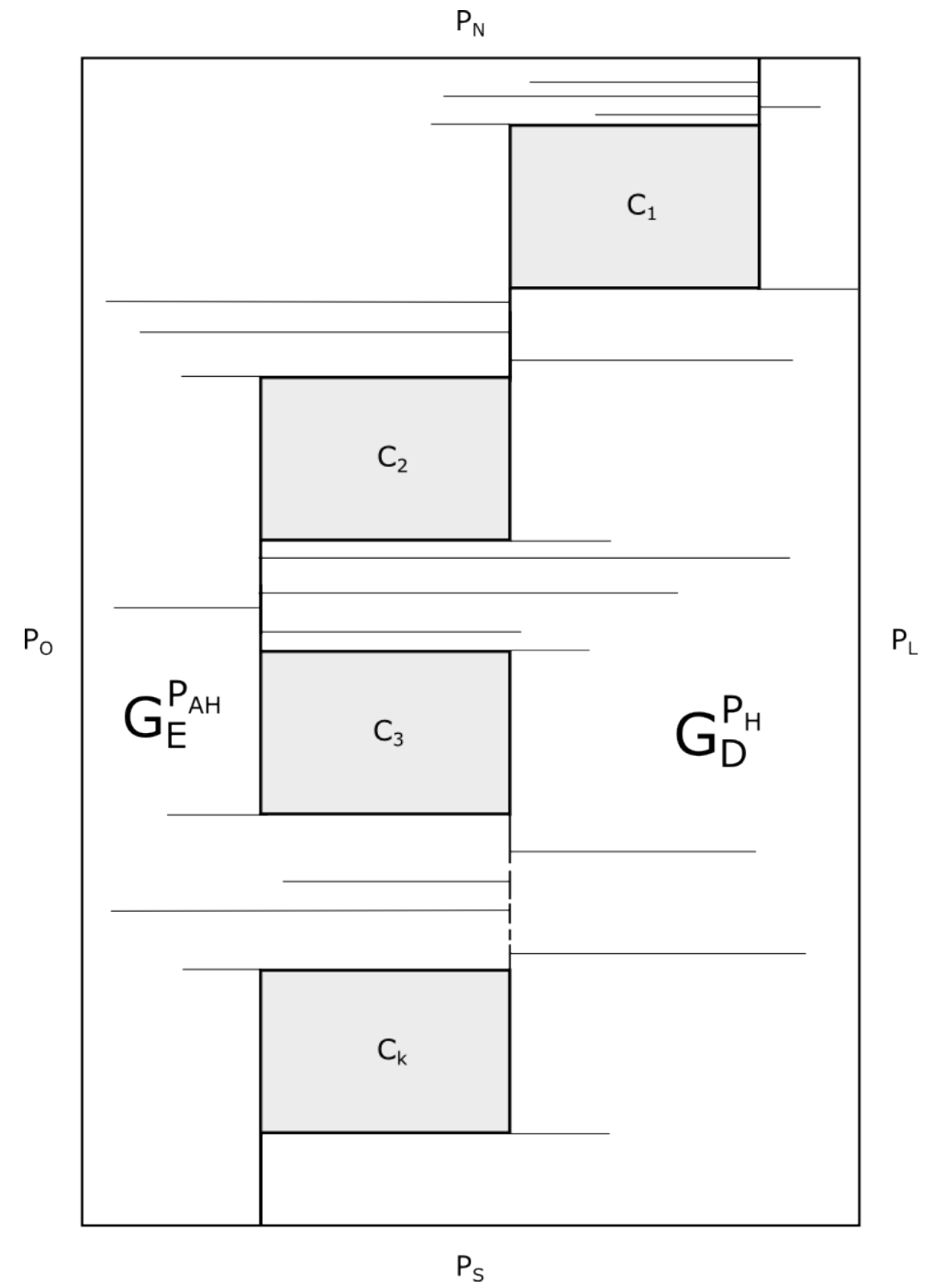

Figura 10.8: Ilustração de uma imersão planar de $P_{H}, P_{A H}$ e dos circuitos $C_{1}, C_{2}, \ldots, C_{k}$ [RNN98].

existe somente uma única perna anti-horária em $P_{A H}^{\prime}$. Portanto, é fácil ver que para estender o desenho retangular de $G_{1}$ basta considerar as mesmas orientações das arestas de $G_{1}$ em $\mathbb{G}$.

Podemos fazer de modo análogo a construção anterior para o grafo $G_{D}^{P_{H}}$. A figura 10.9 ilustra o grafo original, os caminhos $P_{H}$ e $P_{A H}$, os circuitos $C_{1}, C_{2}, \ldots, C_{k}$ e os grafos $G_{1}$ e $G_{2}$. Portanto, se $\mathbb{G}$ possui um desenho retangular, precisamos verificar se existe um desenho retangular de $G_{1}, G_{2}$ e de todos os grafos internos dos circuitos $C_{1}, C_{2}, \ldots, C_{k}$. Assim, se existe dois caminhos $P_{H}$ e $P_{A H}$ de modo que $G_{E}^{P_{A H}^{\prime}}$ e $G_{D}^{P_{H}^{\prime}}$ não possuam componentes ruins então existe um desenho retangular de $\mathbb{G}$, vamos chamar um par de caminhos que satisfaçam estas condições de par particionador. No caso de $P_{H}=P_{A H}$, temos um par particionador simples.

A figura 10.9A, o caminho $P$ (em negrito) é o NS-caminho mais à esquerda. O caminho $P_{A H}$ é ilustrado pelas linhas pontilhadas vermelhas, já o caminho $P_{H}$ é ilustrado pelas linhas 
pontilhadas azuis.

Na figura 10.9B ilustra os subgrafos $G_{E}^{P_{A H}}$ e $G_{D}^{P_{H}}$, juntamente com os dois grafos internos ao circuitos $C_{1}$ e $C_{2}$.

As figuras 10.9C e 10.9D ilustram os grafos $G_{1}$ e $G_{2}$, respectivamente. Estes grafos são resultantes de $G_{E}^{P_{A H}}$ e $G_{D}^{P_{H}}$ após a contração das arestas horizontais de $P_{A H}$ e $P_{H}$.

Agora, nosso problema é encontrar de forma eficiente um par particionador para o grafo $\mathbb{G}$. O princípio que Rahman et al. utiliza é dividir o problema de modo que um dos lados seja fácil de ser resolvido. Assim, de forma incremental encontramos, ou um certificado que não existe um desenho retangular de $\mathbb{G}$ ou teremos um desenho retangular de $\mathbb{G}$. Para isso, vamos encontrar um NS-caminho mais a esquerda possível. Seja $P_{N}=\left\langle v_{0}, v_{1}, v_{2}, \ldots, v_{p}\right\rangle$ e $P_{S}=\left\langle u_{0}, u_{1}, u_{2}, \ldots, u_{q}\right\rangle$. Note que $v_{0}$ está a esquerda de $v_{p}$ e que $u_{0}$ está a direita de $u_{q}$ (figura 10.9A). Um NS-caminho mais a esquerda $P$ é um caminho de satisfaz:

- $P$ inicia no vértice $v_{1}$,

- $P$ termina no vértice $u_{q-1}$,

- $P$ minimiza o número de arestas em $G_{E}^{P}$.

Um modo eficiente para encontrar um NS-caminho mais à esquerda é realizar uma busca em profundidade anti-horária a partir de $v_{1}$. Dado um vértice $w$, uma busca em profundidade anti-horária irá avaliar o próximo vértice adjacente a $w$ no sentido anti-horário e que ainda não foi visitado.

Um NS-caminho mais à esquerda $P$ pode possuir circuitos anexados a ele. É fácil ver que estes circuitos devem estar anexados no sentido horário a $P$. Se observarmos os circuitos que estão anexados a $P$, ou um está estritamente interno a outro circuito, ou estes circuitos são totalmente disjuntos. Digamos que um circuito $C$ é maximal se não existe um outro circuito $C^{\prime}$ de modo que $C$ seja estritamente interno a $C^{\prime}$. A figura 10.10 ilustra a estrutura hierárquica dos circuitos de $\mathbb{G}$. O NS-caminho mais a esquerda $P$ é representado por uma linha vertical e o interior dos sete circuitos críticos $C_{m 1}, C_{m 2}, \ldots, C_{m 7}$ possui a cor cinza.

Para o próximo lema é importante lembrar que as arestas incidentes em uma face estão listadas em ordem horária, ou seja, os circuitos que descrevem as faces estão descritos em ordem horária. Note também que as arestas não são orientadas.

Lema 10.7: Se não existe uma componente ruim em $\mathbb{G}$ e não existe nenhum dos $N S$-, $S N$-, LO- ou $O L$-caminhos da borda, então $\mathbb{G}$ possui um par particionador $P_{H}$ e $P_{A H}$. 
Demonstração: Vamos supor que não existe uma componente ruim em $\mathbb{G}$ e que não existe nenhum dos $N S$-, $S N$-, $L O$ - ou $O L$-caminhos da borda. Vamos mostrar como construir um par particionador $P_{H}$ e $P_{A H}$ a partir do NS-caminho mais a esquerda $P=\left\langle v_{0}, v_{1}, \ldots, v_{k}\right\rangle$.

Inicialmente, vamos encontrar dois caminhos $P_{i n i}$ e $P_{f i m}$. Estes caminhos serão, respectivamente, o segmento inicial e final compartilhado por ambos os caminhos $P_{H}$ e $P_{A H}$.

Considere $i$ como sendo o maior índice de modo que $v_{i-1} v_{i} \in E(P)$ e que $v_{i} v_{i-1}$ pertence a, ou um NN-caminho da borda ou um LN-caminho da borda. Assim, existe uma face incidente ao caminho $P_{N}$ de modo que a aresta $v_{i} v_{i-1}$ pertence a esta face. Seja $Q=\left\langle v_{i}, v_{i-1}=\right.$ $\left.w_{1}, w_{2}, \ldots, w_{l-1}, w_{l}\right\rangle$ o segmento do circuito desta face de modo que inicia pelo vértice $v_{i} \mathrm{e}$ termina no vértice $w_{l}$, onde $w_{l}$ é o único vértice de $Q$ que pertence a $P_{N}$. Escolha $P_{i n i}=$ $\left\langle w_{l}, w_{l-1}, \ldots, w_{2}, w_{1}=v_{i-1}, v_{i}\right\rangle$.

Agora, considere $j$ como sendo o menor índice de modo que $v_{j} v_{j+1} \in E(P)$ e que $v_{j+1} v_{j}$ pertence a, ou um SS-caminho da borda ou um SL-caminho da borda. Assim, existe uma face incidente ao caminho $P_{S}$ de modo que a aresta $v_{j+1} v_{j}$ pertence a esta face. Seja $R=$ $\left\langle x_{1}, x_{2}, \ldots, x_{r-1}, x_{r}=v_{j+1}, v_{j}\right\rangle$ o segmento do circuito desta face de modo que inicia pelo vértice $x_{1}$ e termina no vértice $v_{j}$, onde $x_{1}$ é o único vértice de $R$ que pertence a $P_{S}$. Escolha $P_{\text {fim }}=\left\langle v_{j}, v_{j+1}=x_{r}, \ldots, x_{2}, x_{1}\right\rangle$.

Note que $i \leq j$, caso contrário existiria um SN-caminho da borda, contrariando a hipótese.

Ainda, pela escolha de $P_{i n i}$ e $P_{f i m}$, nem $G_{E}^{P^{\prime}}$ ou $G_{D}^{P^{\prime}}$ possui um circuito que viola o lema 10.4 para qualquer caminho $P^{\prime}$ de modo que $P^{\prime}$ inicia com o caminho $P_{\text {ini }}$ e termina com o caminho $P_{\text {fim }}$. Caso contrário existe uma contradição com a suposição de que $\mathbb{G}$ não possui componentes ruins.

Agora, considere um segmento $P^{\prime}$ de $P$, tal que $P^{\prime}=\left\langle v_{i-1}, v_{i}, \ldots, v_{j}, v_{j+1}\right\rangle$.

Primeiramente, precisamos saber como encontrar todos os circuitos críticos maximais anexados a $P^{\prime}$. Para encontrar todos os circuitos críticos horários anexados a $P^{\prime}$, precisamos percorrer todas as arestas das faces incidentes a $P^{\prime}$. Após percorrermos as faces, todas as arestas que forem percorridas duas vezes são pernas de um circuito crítico horário. Precisamos mostrar agora qual é o circuito crítico horário que esta aresta é perna.

Seja $u v$ uma aresta que estamos passando pela segunda vez. Como percorremos as arestas do circuito que define uma face no sentido horário, na primeira vez que a aresta uv foi percorrida, utilizamos o caminho $C_{1}=\left\langle\ldots, v, u, w_{a}, \ldots, w_{1}\right\rangle$ que descrevia uma face e $w_{1}$ é o único vértice de $C_{1}$ que pertence a $V\left(P^{\prime}\right)$. Agora, considere a face que estamos percorrendo. Utilizamos um caminho $C_{2}=\left\langle x_{1}, \ldots, x_{b}, u, v\right\rangle$, onde $x_{1}$ é o único vértice de $C_{2}$ que pertence a $V\left(P^{\prime}\right)$. Se considerarmos $C=\left\langle w_{1}, \ldots, w_{a}, u, x_{b}, \ldots, x_{1}\right\rangle$, temos que $C$ é um circuito horário anexado a $P^{\prime}$. É fácil ver, pela construção de $C$ que $n_{h}(C)=1, \operatorname{logo} C$ é um circuito crítico. 
Para determinar quais circuitos críticos horários anexados a $P^{\prime}$ são maximais, basta notar que os vértices iniciais e finais de cada circuito obedecem a regra dos parenteses. Logo, o algoritmo para determinar quais circuitos anexados a $P^{\prime}$ são maximais é bem simples.

Considere as arestas de $P^{\prime}$ não estão contidos nenhum circuito crítico horário anexado a $P^{\prime}$. Então, vamos fazer $P_{H}$ e $P_{A H}$ passar por estas arestas, ou seja, estamos definindo alguns dos caminhos compartilhados $P_{1}, P_{2}, \ldots, P_{k}+1$.

Agora, precisamos definir os segmentos de $P_{H}$ e $P_{A H}$ para cada circuito crítico horário maximal $C$ anexado a $P^{\prime}$. (Ilustrados na figura 10.10 pelos circuitos $C_{m 3}, C_{m 4}, C_{m 5} e C_{m 6}$ ) Desde que $n_{H}(C) \leq 1$, temos os seguintes casos:

Caso $1\left(n_{H}(C)=0\right)$ : Neste caso, $n_{A H}(C)>1$. Caso contrário, $\mathbb{G}$ possui uma componente ruim. Assim, $P_{H}$ e $P_{A H}$ devem passar através de $Q_{H}(C)$. (Caso ilustrado no circuito $C_{m 3}$ da figura 10.10)

Caso $2\left(n_{H}(C)=1\right.$ e $\left.n_{A H}(C) \leq 1\right)$ : Neste caso, $n_{A H}(C)=1$. Caso contrário, $\mathbb{G}$ possui uma componente ruim. Assim, $C$ possui quatro pernas. O caminho $P_{H}$ passa através de $Q_{H}(C)$ e $P_{A H}$ passa por $Q_{A H}(C)$. Ainda, $C$ é um dos circuitos de $E\left(P_{H}\right) \oplus E\left(P_{A H}\right)$. (Caso ilustrado no circuito $C_{m 4}$ da figura 10.10).

Caso $3\left(n_{H}(C)=1\right.$ e $\left.\left.n_{A H}(C)>1\right)\right)$ : Neste caso, existem dois casos: (i) não existe, em $\mathbb{G}_{\text {int }}(C)$, um circuito crítico anti-horário anexado a $Q_{H}(C)$ e (ii) existe, em $\mathbb{G}_{\text {int }}$, ao menos um circuito crítico anti-horário anexado a $Q_{H}(C)$. Para o caso (i), os caminhos $P_{H}$ e $P_{A H}$ devem passar através de $Q_{H}(C)$. (Caso ilustrado no circuito $C_{m 5}$ da figura 10.10).

Para o caso (ii), é fácil ver que existe somente um circuito crítico anti-horário $C^{\prime}$ anexado a $Q_{H}(C)$. Caso contrário, se existir mais que um circuito crítico teremos um circuito em $\mathbb{G}$ somente com três pernas, contrariando a hipótese que $\mathbb{G}$ não possui componentes ruins.

Ainda, $n_{A H}\left(C^{\prime}\right)=1$. Caso contrário, se $n_{A H}\left(C^{\prime}\right)=0$, então $C^{\prime}$ possui três pernas.

Assuma que os caminhos $P_{H}$ e $P_{A H}$ passam pelas arestas $E\left(Q_{H}(C)\right)-E\left(C^{\prime}\right)$. O caminho $P_{H}$ passa por $Q_{H}\left(C^{\prime}\right)$ e o $P_{A H}$ passa por $Q_{A H}\left(C^{\prime}\right)$. Assim, $C^{\prime}$ é um dos circuitos induzidos por $E\left(P_{H}\right) \oplus E\left(P_{A H}\right)$.

Portanto, se escolhermos $P_{H}$ e $P_{A H}$ conforme explicamos anteriormente, então todo circuito de $E\left(P_{H}\right) \oplus E\left(P_{A H}\right)$ possui, exatamente, quatro pernas, onde duas destas são compartilhadas por $P_{H}$ e $P_{A H}$. Ainda $G_{E}^{P_{A H}}$ e $G_{D}^{P_{H}}$ não possuem uma componente ruim. Assim, $P_{H}$ e $P_{A H}$ é um par particionador de $\mathbb{G}$.

Assim, podemos enunciar o principal teorema de Rahman et al. O teorema a seguir exibe condições suficientes e necessárias para que um grafo possua um desenho retangular. Este teorema é análogo ao teorema de Thomassen [Tho84]. 
Teorema 10.8: Seja $\mathbb{G}$ um grafo plano de modo que todos os vértices possuem grau 3 exceto os quatro vértices de grau 2 que dividem o circuito externo $C_{0}$ em quatro caminhos $P_{N}, P_{S}, P_{L}$ e $P_{O}$. Então $\mathbb{G}$ possui um desenho retangular se e somente se $\mathbb{G}$ não possui uma componente ruim.

Demonstração: A prova da necessidade é consequência imediata dos lemas 10.2, 10.3 e 10.4. A prova da suficiência é consequência imediata dos lemas 10.5, 10.6 e 10.7.

\subsection{Algoritmo PARA O PROBlEMA DE DESENHO RETANGULAR}

Os resultados da seção anterior implicam em um algoritmo de complexidade linear para solucionar os problemas de decisão e construção do desenho retangular. O algoritmo que iremos apresentar nesta seção encontra, caso existir, um desenho retangular de um grafo plano $\mathbb{G}$. Este algoritmo pode ser facilmente modificado a fim de identificar se o grafo não possui tal desenho.

Assim, o algoritmo recebe como entrada um grafo plano $\mathbb{G}$ e a saída do algoritmo será a orientação (horizontal ou vertical) de cada aresta de $\mathbb{G}$. A partir destas orientações é possível encontrar as coordenadas inteiras de cada vértice de $\mathbb{G}$. O algoritmo para determiná-las será apresentado futuramente.

Algoritmo Desenho-Retangular $(\mathbb{G})$

ENTRADA: Grafo plano $\mathbb{G}$.

SAÍDA: Desenho retangular de $\mathbb{G}$.

1. Defina as arestas do circuito $C_{0}(\mathbb{G})$ de modo que forme um retângulo onde seus lados são os caminhos: $P_{N}, P_{S}, P_{L}$ e $P_{O}$. As arestas de $P_{N}$ e $P_{S}$ são horizontais e as arestas de $P_{L}$ e $P_{O}$ são verticais;

2. Encontre todas as $\mathrm{C}_{0}$-componentes, digamos, $H_{1}, H_{2}, \ldots, H_{k}$;

3. Para cada $C_{0}$-componente $H_{i}$ faça

4. $G_{i} \leftarrow C_{0} \cup H_{i}$;

5. $\operatorname{Desenhe}\left(G_{i}, H_{i}\right)$;

A seguir, vamos apresentar uma versão simplificada do algoritmo DeSEnHE. Esta versão não utiliza memoização. As modificações necessárias para a implementação da memoização será apresentada futuramente. 


\section{Algoritmo Desenhe ( $\mathbb{G}, H)$}

Entrada: Grafo plano $\mathbb{G}$ e uma $C_{0}(\mathbb{G})$-componente $H$.

SAÍDA: Desenho retangular da $C_{0}(\mathbb{G})$-componente $H$ com o circuito $C_{0}(\mathbb{G})$ fixo.

/* $H$ é uma $C_{0}$-componente do grafo $\mathbb{G} * /$

1. Se $\mathbb{G}$ possui um NS-, SN-, OL-, ou LO-caminho da borda $P$ então /* P é um caminho particionador */

2. Sem perda de generalidade, assuma que $P$ é um NS-caminho da borda;

3. Defina, para cada aresta $e \in E(P)$, que $e$ é vertical;

4. $\quad$ Se $|E(P)| \geq 2$ então cada

6. Sejam $F_{1}, F_{2}, \ldots, F_{k}$ as $C_{0}$-componentes de $G_{D}^{P}$, dado que as direções de

7.

8. Senão

9. Encontre o NS-caminho mais a esquerda $P$;

10. Encontre um par particionador $P_{H}$ e $P_{A H}$ a partir de $P$ conforme descrito no lema 10.7;

11. Se $P_{H}=P_{A H}$ então

12. Defina, para cada aresta $e \in E\left(P_{H}\right)$, que $e$ é vertical;

13. Seja $G_{1}=G_{E}^{P_{H}}$ e $G_{2}=G_{D}^{P_{H}}$ dois subgrafos de $\mathbb{G}$ de modo que os circuitos externos $C_{0}\left(G_{E}^{P_{H}}\right)$ e $C_{0}\left(G_{E}^{P_{H}}\right)$ possuem uma imersão planar retangular já fixada;

14. Para cada $G_{i}$ faça

15. Seja $F_{1}, F_{2}, \ldots, F_{k}$ as $C_{0}$-componentes de $G_{i}$;

16. Para cada $F_{j}$ faça

17. $\operatorname{Desenhe}\left(F_{j}, C_{0}\left(G_{i}\right) \cup F_{j}\right)$;

18. Senão

19. Defina para todas as arestas de $P_{H}$ e $P_{A H}$ segmentos horizontais e verticais alternados conforme ilustra a figura 10.8 ;

20. Seja $G_{1}$ ○ grafo obtido a partir de $G_{E}^{P_{A H}}$ contraindo todas as arestas horizontais de $P_{A H}$ que pertencem a um dos lados horizontais das imersões planares retangulares de $C_{1}, C_{2}, \ldots, C_{k}$;

21.

Seja $G_{2}$ o grafo obtido a partir de $G_{D}^{P_{H}}$ contraindo todas as arestas horizontais de $P_{H}$ que pertencem a um dos lados horizontais das imersões planares retangulares de $C_{1}, C_{2}, \ldots, C_{k}$;

22.

Seja $G_{3}=\mathbb{G}_{\text {int }}\left(C_{1}\right), G_{4}=\mathbb{G}_{i n t}\left(C_{2}\right), \ldots, G_{k+2}=\mathbb{G}_{i n t}\left(C_{k}\right)$ os subgrafos definidos a partir das imersões planares retangulares dos circuitos $C_{1}, C_{2}, \ldots, C_{k}$; 
23.

24.

25.

26.

\section{Para cada $G_{i}$ faça}

Seja $F_{1}, F_{2}, \ldots, F_{k}$ as $C_{0}$-componentes de $G_{i}$;

\section{Para cada $F_{j}$ faça}

$\operatorname{Desenhe}\left(F_{j}, C_{0}\left(G_{i}\right) \cup F_{j}\right)$;

É fácil ver que este algoritmo implementa os resultados apresentados na seção anterior.

Precisamos mostrar que o algoritmo Desenho-Retangular possui complexidade linear. Para isso, mostraremos que vamos percorrer cada face um número constante de vezes.

Inicialmente, precisamos encontrar todas as $C_{0}$-componentes de $\mathbb{G}$ para verificar se existe algum NS-, SN-, OL-, ou LO-caminho da borda. Faremos isso utilizando uma busca em profundidade anti-horária. Durante a busca, toda aresta que pertence a uma borda é rotulada por um dos rótulos: $N N, N O, \ldots, L L$. O rótulo é definido a partir do vértice que inicia e termina o caminho da borda, por exemplo, se o primeiro vértice do caminho da borda pertence a $P_{N}$ e o último vértice a $P_{O}$, então todas as arestas do caminho devem receber o rótulo NO. Assim, se existir algum NS-, SN-, OL-, ou LO-caminho da borda, podemos obter este em tempo constante a partir dos rótulos das arestas.

Caso não exista algum NS-, SN-, OL-, ou LO-caminho da borda, precisamos encontrar o NS-caminho mais a esquerda $P$. Iremos encontrá-lo utilizando uma busca em profundidade anti-horária em todos os caminhos de borda que iniciam e terminam em $P_{O}$. Para facilitar este procedimento, basta analisar os rótulos das arestas que já foram previamente definidos. Após isto, podemos encontrar um par particionador $P_{H}$ e $P_{A H}$ a partir de $P$ conforme descrito no lema 10.7. Seja $S$ um segmento de $P$ que conecta os vértices $e_{a}$ e $e_{b}$. Se percorrermos o contorno de todos os circuitos das faces anexados a $S$ no sentido horário podemos encontrar todos os circuitos críticos horários anexados a $S$, caso estes existam. Conforme já apresentado anteriormente, uma perna de um circuito crítico é uma aresta que não incide em nenhum vértice de $S$ e foi percorrida duas vezes durante a busca para encontrar o circuito crítico.

Podemos observar que os vértices que iniciam o terminam os circuitos críticos em $P$ respeitam a regra dos parênteses. Assim, podemos encontrar todos os circuitos críticos horários maximais anexados a $P$. Logo, podemos encontrar o par particionador $P_{H}$ e $P_{A H}$ a partir de $P$. Este procedimento percorre as seguintes arestas em uma quantidade constante de vezes: (i) arestas pertencentes a $P_{H}$ e $P_{A H}$; (ii) arestas dos circuitos das faces que estão anexadas a $P$; e (iii) arestas que pertencem aos circuitos externos dos grafos criados a partir de $P_{H}$ e $P_{A H}$.

Depois de encontrar um par particionador ou um caminho particionador, precisamos atribuir novos rótulos aos caminhos da bordas que foram criados. Assim, os rótulos de alguns caminhos antigos deverão ser atualizados. Claramente, podemos fazer esta atualização percorrendo somente uma vez os novos caminhos da borda. 
Seja $P$ um NS-caminho mais a esquerda $P$ de um subgrafo $\mathbb{G}^{\prime}$ de $\mathbb{G}$. Teremos um problema com a complexidade do algoritmo Desenhe, se um segmento $S$ de $P$, onde $S$ é diferente de $P_{H}$ e $P_{A H}$, é escolhido como um NS-caminho mais a esquerda em uma outra chamada recursiva. Se percorrermos todos os circuitos das faces anexadas a $S$ novamente, então a complexidade do algoritmo não será linear. Para contornar esta dificuldade iremos memoizar algumas informações relativas ao caminho $P$. São elas:

(1) Uma lista de todas as arestas $e_{i} \in E(P)$ que pertencem a um NN- e NL- caminho da borda;

(2) Uma lista de todas as arestas $e_{i} \in E(P)$ que pertencem a um SS- e SO- caminho da borda;

(3) Um vetor de tamanho $|V(P)|$ contendo uma variável booleana indicando se o vértice corresponde ao início ou término de um circuito crítico horário $C$ anexado a $P$ e se $Q_{a h}(C)=1$ ou $Q_{a h}>1$.

Vamos usar as listas (i) e (ii) para encontrar $P_{i n i}$ e $P_{\text {fim }}$ diretamente em algum estágio recursivo. As marcas dos vértices que pertencem a (iii) indicam a existência de circuitos críticos anexados a $S$. Assim, quando escolhermos $S$ como um NS-caminhos mais a esquerda, não precisaremos encontrar todos os circuitos críticos novamente.

Para implementar a memoização, precisamos utilizar uma estrutura auxiliar que deve ser passada como parâmetro. Esta estrutura deve conter os itens (i), (ii) e (iii) e uma variável que indique se o NS-caminho mais a esquerda do subgrafo que estamos processando é segmento de algum NS-caminho mais a esquerda que já foi processado. Perceba que quando escolhemos o par particionador ou um caminho particionador dividimos em subgrafos que podem possuir segmentos do caminho $P$ como seu NS-caminho mais a esquerda, logo, podemos decidir no momento da chamada recursiva se os dados do NS-caminho mais a esquerda já foram processados.

Desta forma, durante a execução do algoritmo, toda face de $\mathbb{G}$, em algum momento, será uma face da borda de algum subgrafo de $\mathbb{G}$. Ainda, uma vez que ela se tornou uma face da borda, ela sempre será uma face da borda.

Portanto, se utilizarmos a memoização, percorreremos as arestas do circuito de cada face de $\mathbb{G}$ um número constante de vezes, implicando que o algoritmo Desenho-Retangular possui complexidade linear. 


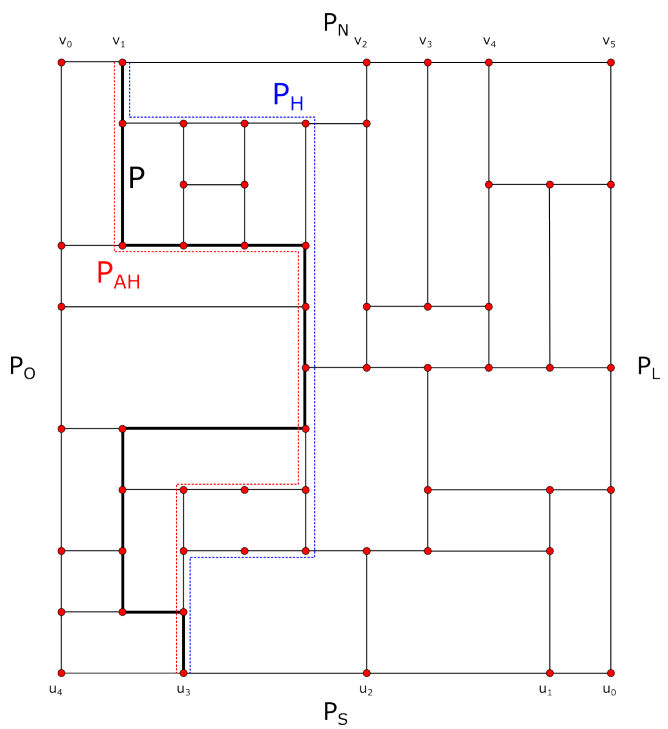

(A)
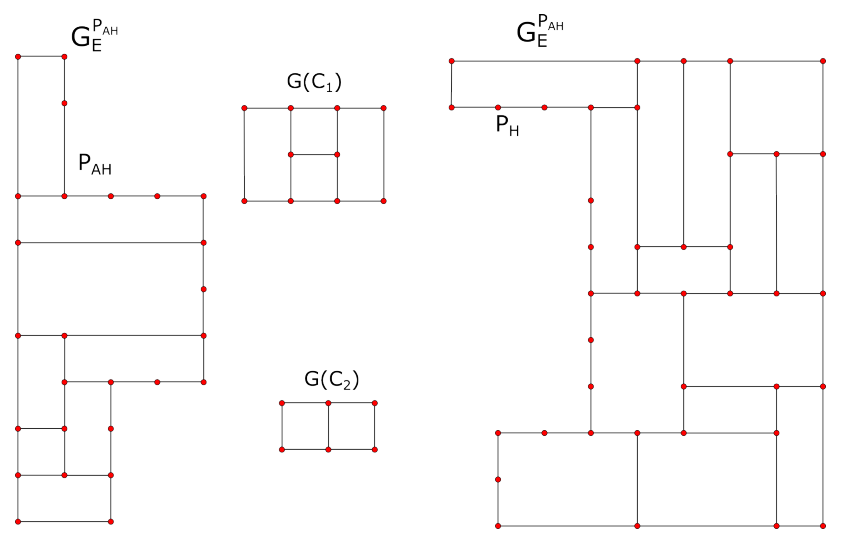

(B)

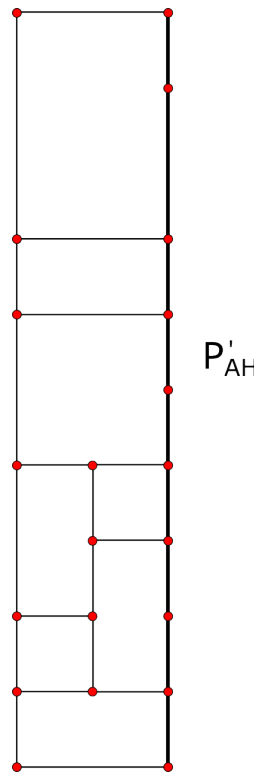

(C) $G_{1}$

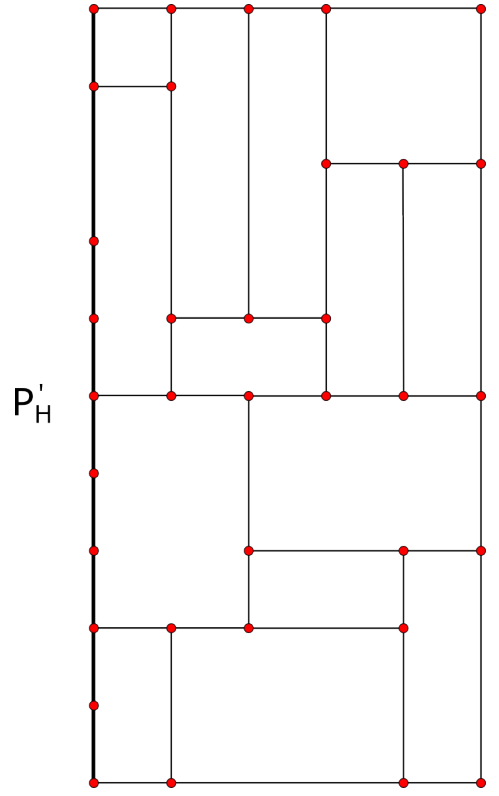

(D) $G_{2}$

Figura 10.9: Ilustração de uma imersão planar de um par particionador[RNN98]. 


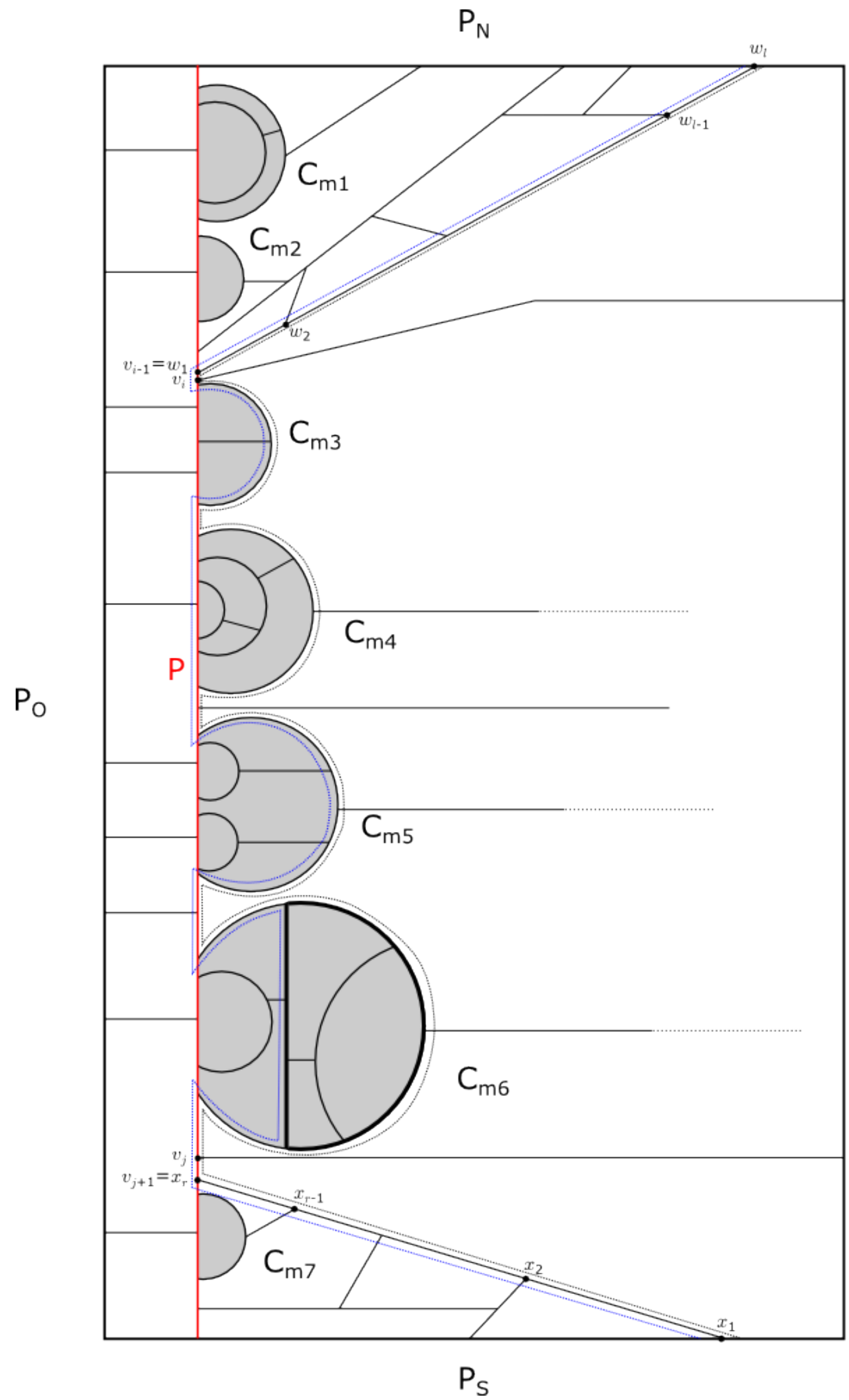

Figura 10.10: Ilustração dos circuitos críticos horários anexados ao NS-caminho mais a esquerda $P$ [RNN98]. 



\section{Algoritmo PARA DETERMINAR AS COORDENADAS DOS VÉRTICES DO GRAFO RETANGULAR}

Existem diversas técnicas para solucionar os problemas que envolvem a construção da representação ou do desenho retangular (problemas 2 e 4), entretanto, a maioria das técnicas procuram encontrar as orientações das arestas, horizontal ou vertical, do grafo de entrada de modo a existir uma representação (desenho) retangular. Nesta seção, vamos assumir que estamos resolvendo o problema de desenho retangular. Também vamos assumir que o grafo de entrada possui um desenho retangular e sabemos as orientações de todas as arestas do grafo. Logo, nosso problema é em determinar as coordenadas dos vértices para representar o grafo em um plano.

Um trabalho que se preocupa com tal procedimento é o desenvolvido por Rahman et al. [RNN98]. Em [RNN98], os autores procuram minimizar o perímetro ou a área ocupada pelo desenho retangular.

Primeiramente, só faz sentido falar em minimizar o perímetro ou a área de um desenho retangular se adicionarmos a restrição que todo vértice deve possuir coordenadas inteiras e toda aresta deve possuir comprimento maior ou igual a 1. Caso não adicionarmos estas restrições, podemos diminuir o grafo o quanto desejarmos e obter um desenho retangular 
com perímetro e área menores.

Para isso, Rahman et al. apresentam o conceito de desenho retangular compacto na grade. Dizemos que um desenho é compacto na grade se para toda coordenada $x$ existe ao menos uma aresta vertical e para toda coordenada $y$ existe ao menos uma aresta horizontal naquela coordenada.

Nesta seção, vamos apresentar um algoritmo que desenvolvemos baseado em [RNN98] que encontra uma representação retangular compacta na grade. Entretanto, o algoritmo desenvolvido utiliza uma abordagem distinta que o torna mais simples que o apresentado por Rahman et al. O algoritmo de Rahman é um método alternativo aos apresentados em [Tam87, TT89]. Todos estes algoritmos determinam as coordenadas em tempo linear.

\subsection{Algoritmo DE RAHMAN et al. PARA DETERMinAR AS COORDENADAS DOS VÉRTICES}

O algoritmo de Rahman et al. para determinar as coordenadas dos vértices a partir das orientações das arestas atribui separadamente as coordenadas dos eixos $x$ e $y$ (abscissa e ordenadas). Entretanto, o método para determinar as coordenadas $x$ é análogo ao método para determinar as coordenadas $y$. Nesta seção vamos mostrar o método para determinar as coordenadas $y$.

Seja $\mathbb{G}=(V, E)$ um grafo plano. Vamos considerar que as arestas de $\mathbb{G}$ já estão com suas orientações definidas, ou seja, elas já estão, ou na horizontal, ou na vertical. Também assumimos que a origem, o ponto $(0,0)$, é o canto inferior esquerdo do desenho retangular. Ainda, vamos supor que sabemos quais vértices de $V(\mathbb{G})$ são os cantos do desenho retangular, a saber: superior esquerdo, superior direito, inferior esquerdo e inferior direito.

Inicialmente, teremos para cada vértice $v$ de $V(\mathbb{G})$, uma variável temp $(v)$ como um valor temporário da coordenada $y$ para o vértice $v$. O desenho retangular de $\mathbb{G}$ é composto por um conjunto de caminhos horizontais e verticais maximais. Para cada caminho maximal horizontal $C$, vamos definir a coordenada $y$ deste caminho como $y(C)$, logo, todos os vértices que pertencem a $C$ possuem coordenada $y$ igual a $y(C)$.

Considere um vértice $v$ que pertence ao caminho horizontal maximal $C$. Existem dois casos a serem tratados: se $v$ possui um vizinho $u$ que está em um caminho abaixo de $C$ ou se $v$ não possui nenhum vizinho abaixo de $C$. Caso exista um vizinho $u$ abaixo de $v$, então $\operatorname{temp}(v)=y\left(L^{\prime}\right)+1$, onde $L^{\prime}$ é o único caminho horizontal maximal que contém $u$. Caso não exista tal vizinho $u$, então temp $(v)=0$. Por fim, definimos $y(C)=\max _{v \in C}\{\operatorname{temp}(v)\}$, para todo vértice $v$ que pertence a $C$. É fácil ver que o caminho maximal horizontal $P_{S}$ que contém os vértices do canto inferior esquerdo e do canto inferior direito possui $y\left(P_{S}\right)=0$, 
pois temp $(v)=0$ para todo $v$ pertencente a $P_{S}$.

Para computar os valores de $y(C)$, para todo caminho horizontal maximal $C$, Rahman et al. faz uma busca em profundidade em um grafo modificado a partir do vértice que será o canto superior esquerdo $v_{s d}$ do desenho retangular de $\mathbb{G}$. Em $\mathbb{G}$, podem existir três tipos configurações das arestas incidentes em um vértice de modo que alguma aresta vertical será considerada como uma aresta proibida. Estas configurações são representadas na figura 11.1. Em cada configuração, a aresta vertical proibida é a representada por uma linha pontilhada.
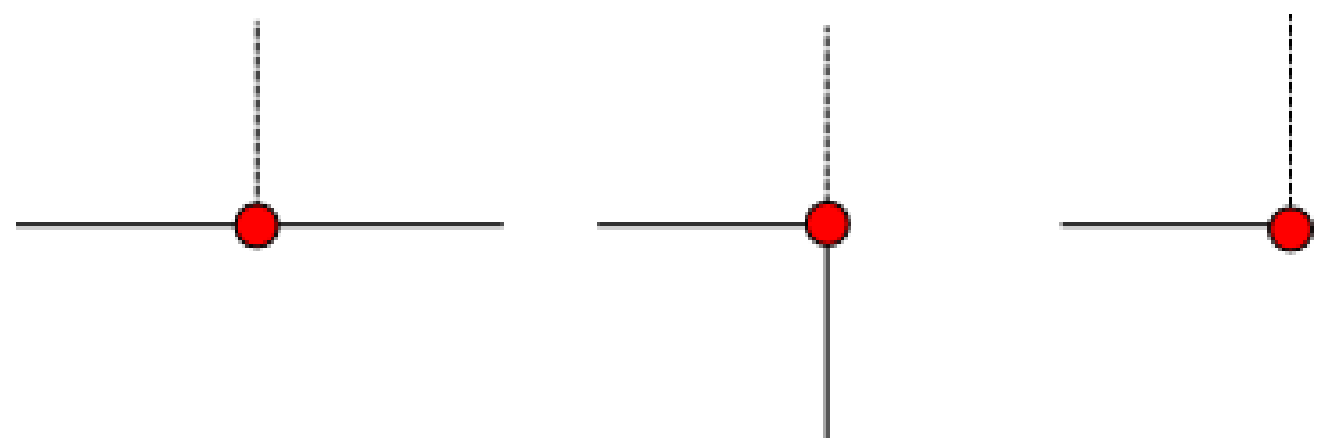

Figura 11.1: Exemplo de configurações proibidas em $\mathbb{G}$. As arestas pontilhadas são as arestas verticais proibidas[RNN98].

Considere um grafo $T_{y}$ obtido de $\mathbb{G}$ onde todas as arestas verticais proibidas são removidas. Rahman et al. perceberam que $T_{y}$ é uma árvore geradora de $\mathbb{G}$, pois toda face de $\mathbb{G}$ contém pelo menos uma aresta vertical que não foi removida, pois não formava uma das configurações apresentada na figura 11.1. Mais precisamente, todas as arestas verticais mais à esquerda de cada caminho maximal horizontal não foi removida. A figura 11.2B representa o grafo $T_{y}$ obtido de $\mathbb{G}$.

Vamos atribuir os valores de $y(C)$ para todo caminho horizontal maximal $C$ de modo que exista uma ordem. A ordem será definida a partir da posição do caminho, o primeiro caminho será o que estiver mais abaixo e o último o que estiver mais acima, ou seja, antes de atribuir um valor para um caminho horizontal maximal $C$ qualquer, devemos atribuir a coordena $y$ para todos os caminhos que estão abaixo de $C$.

Então, vamos fazer uma busca em profundidade no sentido anti-horário em $T_{y}$, iniciando pelo vértice superior esquerdo e pela aresta vertical incidente neste vértice.

Claramente, esse algoritmo atribui as coordenadas $y$ corretas para todos os vértices de $\mathbb{G}$ em tempo linear. E existe uma reta horizontal em cada coordenada $y$. Logo, como determinar as coordenadas $x$ é análogo a este algoritmo, temos uma representação retangular no grade compacta. 


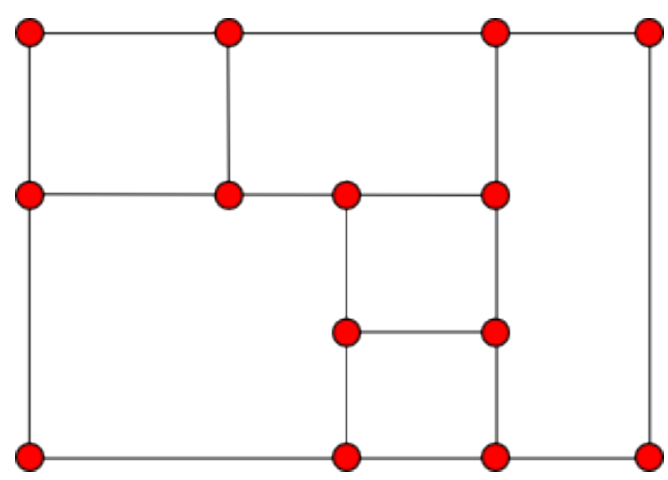

(A)

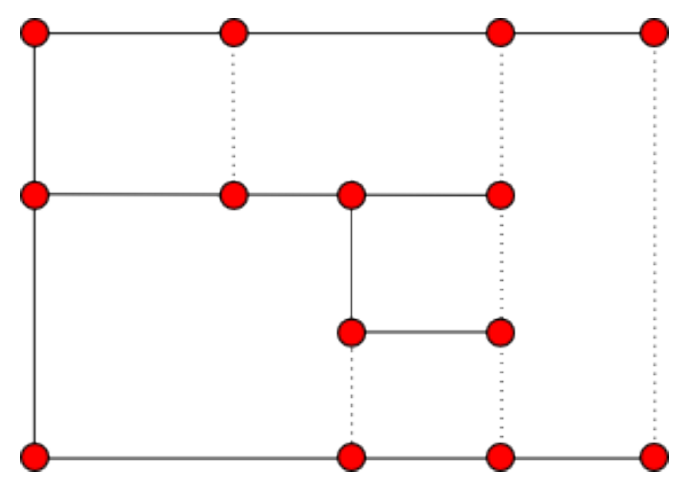

(B)

Figura 11.2: (A) Grafo de entrada $\mathbb{G}$. (B) Ilustração representando $T_{y}$ com linhas grossas.[RNN98].

\subsection{Algoritmo PARA DETERMINAR AS COORDENADAS DOS VÉRTICES BASEADO NA ORDENAÇÃO TOPOLÓGICA DOS CAMINHOS MAXIMAIS}

O algoritmo desenvolvido que utiliza a ordenação topológica é baseado no algoritmo apresentado na seção 11.1. Objetivamos encontrar um desenho retangular compacto na grade e que o algoritmo seja mais simples que o apresentado anteriormente.

Antes de apresentar o algoritmo, vamos introduzir uma estrutura de dados para representar o grafo para o qual desejamos encontrar um desenho retangular. Um fato muito importante que poucos trabalhos exploram é a estrutura geométrica do desenho. Para cada vértice do grafo, temos que existem no máximo três arestas incidentes a ele e estas arestas só podem ter quatro direções distintas: para cima, para baixo, para o lado esquerdo ou para o lado direito.

Como supomos que sabemos quais são os vértices dos respectivos cantos do desenho retangular, então, podemos fazer um pré-processamento no grafo e armazenar, para cada vértice, quais são as arestas incidentes e suas respectivas direções, o que irá consumir complexidade de espaço linear na quantidade de vértices.

Agora vamos explicar o algoritmo desenvolvido. Assim como Rahman et al., determinamos separadamente as coordenadas $x$ e $y$. Vamos mostrar como determinar as coordenadas $y$, entretanto, o algoritmo para determinar as coordenadas $x$ é análogo.

Dado um grafo plano $\mathbb{G}$ o algoritmo consiste em construir um grafo orientado $\mathbb{D}=\left(C, E^{\prime}\right)$, onde cada vértice $c$ em $C$ representa um caminho horizontal maximal e, para cada aresta $u v \in E^{\prime}$, o caminho $u$ está abaixo de $v$ e existe em $\mathbb{G}$ uma aresta de algum vértice do caminho $u$ para algum vértice do caminho $v$.

É fácil ver que o grafo $\mathbb{D}$ não possui ciclos, logo podemos utilizar uma ordenação 
topológica para definir a coordenada $y$ dos vértices de $\mathbb{G}$. O algoritmo é da seguinte forma, para cada vértice $c \in C$ vamos definir uma variável coord $(c)$ que inicialmente é igual a 0 para todo $c$. Vamos mostrar como modificar coord (.) de modo que no final do algoritmo, a variável coord $(c)$ será a coordenada $y$ para todo $w \in c$.

Inicialmente, o vértice com grau de entrada igual a 0 em $\mathbb{D}$ é o vértice que contém o vértice do canto inferior esquerdo, seja este $c$. Como coord $(c)=0$, então $\operatorname{coord}(c)$ contém a coordenada correta do caminho horizontal maximal $c$. Para todo vértice $u$ adjacente a $c$, $\operatorname{vamos} \operatorname{atribuir} \operatorname{coord}(u)=\max \{\operatorname{coord}(c)+1=1, \operatorname{coord}(u)\}$.

Assim, devemos remover o vértice $c$ de $\mathbb{D}$. Como o grafo é acíclico, deve existir um vértice $v$ que possui grau de entrada igual a 0 , vamos repetir o processo que fizemos para $c$, atualizando o valor de coord $(u)$ para todos os vértices vizinhos de $v$. Logo, devemos definir $\operatorname{coord}(u)$ como sendo:

$$
\operatorname{coord}(u)=\max \{\operatorname{coord}(v)+1, \operatorname{coord}(u)\}, \forall v \text { adjacente a } u
$$

Após, devemos remover $v$ e escolher outro vértice com grau de entrada igual a 0. Como temos um conjunto finito de vértices, este processo é finito e após a execução do algoritmo, temos para todo vértice $c \in C$, a coordenada $y$ definida corretamente. De modo análogo, podemos definir as coordenas $x$.

É fácil ver que este algoritmo gera uma representação retangular no grade compacta. A figura 11.3 ilustra o grafo de entrada e o grafo $\mathbb{D}$ correspondente.

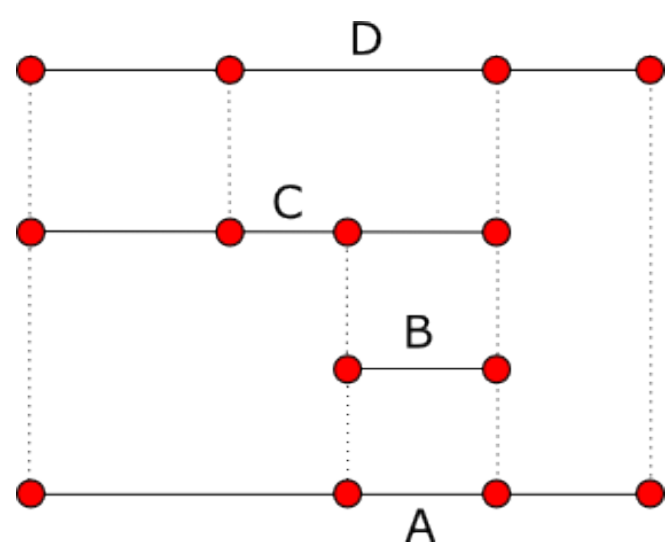

(A)

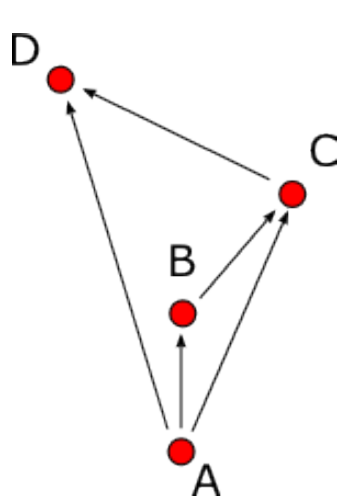

(B)

Figura 11.3: (A) Grafo de entrada $\mathbb{G}$, onde A, B, C e D indicam os caminhos horizontais maximais. (B) Ilustração do grafo $\mathbb{D}$ obtido a partir de $\mathbb{G}$.

\section{LiMitante SUPERIOR PARA O PERÍMETRo E ÁREA MÍNIMA}

Nesta seção vamos enunciar o teorema provado por Rahman et al. em [RNN98]. 
Vamos considerar que a coordenada do canto inferior esquerdo é $(0,0)$ e a coordenada do canto superior direito é $(L, A)$.

Teorema 11.1: O tamanho de qualquer desenho retangular $\mathbb{D}$ compacto na grade de um grafo satisfaz $L+A \leq \frac{n}{2}$ e $L A \leq \frac{n^{2}}{16}$, onde $L$ é a largura e $A$ é a altura do desenho retangular.

Demonstração: Seja $c$ a quantidade de caminhos verticais e horizontais maximais de $\mathbb{D}$.

Pela suposição de que todos os vértices possuem grau 3 a menos os 4 cantos do retângulo externo de $\mathbb{D}$ que possuem grau 2, temos que cada vértice de grau 3 é extremo de exatamente um dos $c-4$ caminhos maximais de $\mathbb{D}$ (retirando os caminhos $P_{N}, P_{S}, P_{O}$ e $P_{L}$ que possuem os vértices de grau 2 como extremo).

Pelo fato de que todo caminho maximal de $\mathbb{D}$ possui dois vértices extremos, temos:

$$
\begin{aligned}
n-4 & =2(c-4) \\
c-2 & =\frac{n}{2} .
\end{aligned}
$$

Seja $c_{H}$ o número de caminhos horizontais maximais e $c_{V}$ o número de caminhos verticais maximais. Como $\mathbb{D}$ é compacto, temos:

$$
\begin{aligned}
A & \leq c_{H}-1 \\
L & \leq c_{V}-1,
\end{aligned}
$$

Portanto,

$$
L+A \leq c_{H}-1+c_{V}-1 \leq c_{H}+c_{V}-2=c-2=\frac{n}{2} .
$$

Agora vamos mostrar que a área do $\mathbb{D}$ é limitada superiormente por $\frac{n^{2}}{16}$.

Pelo resultado apresentado anteriormente, temos:

$$
\begin{aligned}
L+A & \leq \frac{n}{2} \\
A & \leq \frac{n}{2}-L .
\end{aligned}
$$

Assim,

$$
L . A \leq L\left(\frac{n}{2}-L\right)=\frac{n}{2} L-L^{2} .
$$


Deste modo, temos a área em função de $L$. Defini-se $\mathrm{F}(L)=\frac{n}{2} L-L^{2}$.

$$
\begin{aligned}
f^{\prime}(L) & =\frac{n}{2}-2 L, \\
f^{\prime \prime}(L) & =-2 .
\end{aligned}
$$

Logo, como $f^{\prime \prime}(L)<0$, para todo $L$ então a concavidade da função é voltada para baixo, logo quando $f^{\prime}(L)=0$, temos um ponto máximo.

$$
\begin{aligned}
f^{\prime}(L) & =0 \\
\frac{n}{2}-2 L & =0 \\
L & =\frac{n}{4} .
\end{aligned}
$$

Portanto, temos que a área de $\mathbb{D}$ é máxima quando:

$$
A L \leq \frac{n}{4} \frac{n}{4}=\frac{n^{2}}{16}
$$

Rahman et al. afirmam que este limitante é justo, mostrando que é possível construir um conjunto infinito de exemplos que as coordenadas são exatamente iguais ao limitante superior. Um destes exemplos pode ser visualizado na figura 11.4. 


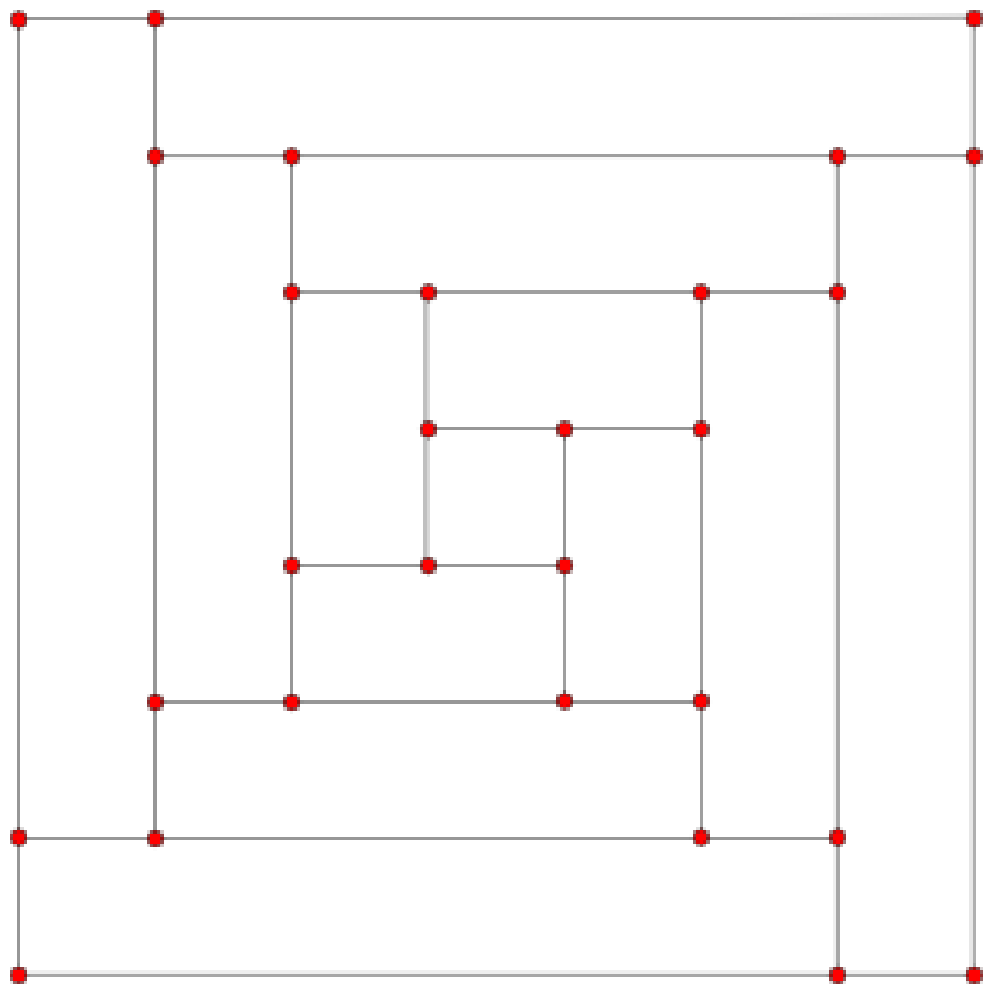

Figura 11.4: Exemplo de uma representação retangular no grade compacta no qual o limitante superior é justo[RNN98]. 


\section{2 \\ CONCLUSÕES}

\subsection{CONSIDERAÇÕes Finais}

Neste trabalho foram apresentados resultados para os problemas de representação retangular e do desenho retangular.

Inicialmente, nosso intuito era de estudar somente o problema de decisão e construção associado à representação retangular de um grafo plano. Entretanto, com o desenvolvimento do trabalho nos deparamos com alguns resultados para um problema muito semelhante ao da representação retangular, o problema do desenho retangular.

A partir deste momento, percebemos que os resultados existentes não possuíam uma nomenclatura única. Buscamos assim, definir uma notação e reescrever os principais resultados para os problemas que estão relacionados a representação retangular e ao desenho retangular. Cada um dos trabalhos que foram apresentados possui alguma característica interessante.

O trabalho de Koźmiński e Kinnen, apresentado no capítulo 4, se destaca pela caracterização do problema de representação retangular. É fácil ver que existe um algoritmo de complexidade cúbica, no número de vértices, para verificar a existência de algum triângulo que não é face, porém Koźmiński e Kinnen utilizam a estrutura do problema e apresentam um algoritmo quadrático. 
No capítulo 5 apresentamos o algoritmo de Bhasker e Sânio que possui complexidade linear e utiliza um grafo dos caminhos dirigidos. O algoritmo que encontra uma representação retangular a partir de um "grafo dos caminhos dirigidos" do grafo da entrada é muito simples, entretanto, encontrar um grafo dos caminhos dirigidos não é trivial e sua implementação possui muitos casos especiais de devem ser tratados.

No capítulo 6 apresentamos o algoritmo de He que utiliza s,t-caminhos. Entretanto, o algoritmo depende diretamente de uma numeração consistente das faces das s,t-redes. Ainda, dada duas numerações consistentes, temos que as respectivas representações retangulares são distintas. Entretanto, não é trivial visualizar se existe algum método que torne viável a construção de todas as representações retangulares de um determinado grafo.

No capítulo 7 apresentamos o trabalho de Lai e Leinwand que se destaca por ser uma elegante redução do problema de representação retangular ao problema de encontrar um emparelhamento perfeito em grafos bipartidos. Mesmo não resultando em um algoritmo linear este algoritmo possui uma relevância prática, pois é possível obter todas as representações retangulares do grafo da entrada.

A possibilidade de gerar todas as representações retangulares é uma propriedade particular do algoritmo de Lai e Leinwand, pois todos os outros métodos dependem de algum fato para construir uma representação retangular. Por exemplo, o algoritmo de Bhasker e Shani depende diretamente do grafo dos caminhos dirigidos. O algoritmo que utilizamos para obter um grafo dos caminhos dirigidos não obtém todas as possibilidades, pois para tratar os casos especiais o algoritmo toma algumas decisões que restringem algumas configurações.

No capítulo 9 apresentamos a caracterização de Thomassen para o problema de desenho retangular. Entretanto, Thomassen não apresenta um algoritmo.

Por fim, no capítulo 10 apresentamos o algoritmo de Rahman et al. para o problema de desenho retangular. O desenho retangular obtido por este algoritmo depende diretamente de como serão escolhidos os caminhos particionadores. Entretanto, podemos modificar a forma com que os circuitos críticos serão desenhados no desenho retangular. Porém, não nos parece algo trivial de ser realizado.

Finalmente, devemos destacar mais uma contribuição deste trabalho: o algoritmo para determinar as coordenadas dos vértices quando sabemos quais arestas são horizontais ou verticais. Como já falado anteriormente, a maioria dos trabalhos não menciona a necessidade de ter que definir as coordenadas dos vértices. Podemos conjecturar que isto se deve às características dos trabalho, no sentido de o quanto estes se preocuparam em desenvolver uma implementação computacional. Dizemos isto, pois quando implementamos os algoritmos, determinar as coordenadas é um problema subsequente, e este não pode ser ignorado.

Neste contexto, nossa abordagem para determinar as coordenadas dos vértices é um 
método tão eficiente quanto os existentes, entretanto a consideramos mais simples.

\subsection{Sugestões Para Pesquisas Futuras}

Inicialmente, vamos fazer algumas sugestões pontuais referentes aos métodos apresentados neste trabalho.

Como podemos observar, a existência de algoritmos lineares impossibilita o desenvolvimento de algum método mais eficiente, pois sabemos que o precisamos ao menos percorrer todas as arestas do grafo. Logo, em termos de complexidade, ainda é possível melhorar as constantes envolvidas.

O desenvolvimento de novos algoritmos para o problema de representação retangular é muito interessante, entretanto, acreditamos que existe a necessidade de um algoritmo que encontre todas as representações retangulares.

Uma sugestão é o desenvolvimento de um algoritmo que combine um método linear e o algoritmo de Lai e Leinwand. A ideia deste algoritmo seria encontrar uma representação retangular em tempo linear e construir um grafo bipartido conforme descrito por Lai e Leinwand. A partir daí, seria possível iterar sobre todas as representações retangulares utilizando os caminhos alternantes do emparelhamento no grafo bipartido.

Ainda com o objetivo de desenvolver um algoritmo para construir todas as representações retangulares, seria interessante modificar o algoritmo Rahman et al. para obter um novo desenho retangular a partir do desenho atual.

Uma outra possibilidade é o estudo da quantidade de representações retangulares distintas que um grafo pode possuir.

Outra sugestão é tentar simplificar o algoritmo de Bhasker e Shani ou até mesmo criar um novo algoritmo para encontrar um grafo dos caminhos dirigidos de um grafo plano triangulado. Pois, como apresentado, a implementação do apresentado neste trabalho não é trivial e possui muitos casos a serem tratados.

Por último, acreditamos que os algoritmos para solucionar os problemas de decisão e construção que envolvem representações retangulares podem auxiliar no desenvolvimento de métodos para problemas de otimização associados à representação retangular. 



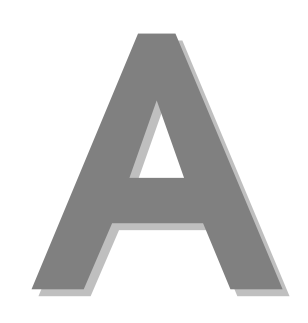

\section{IMPLEMENTAÇÃO DO ALGORITMO PARA CONSTRUIR UM GRAFO DOS CAMINHOS} DIRIGIDOS

O algoritmo que apresentamos no capítulo 5 não possui uma implementação eficiente direta. Existem alguns detalhes que se não implementados de forma correta, fazem com que o algoritmo não tenha complexidade linear.

Vamos dividir a apresentação da implementação em duas partes: Variáveis e algoritmo.

A primeira parte consiste em uma listagem das variáveis do algoritmo, onde realizamos uma descrição de cada variável. Esta seção irá servir como referência para a segunda parte, aonde apresentamos as funções que encontram um grafo dos caminhos dirigidos.

\subsection{VARIÁVEIS}

O algoritmo que iremos apresentar utiliza, além do grafo de entrada $\mathbb{G}$, um outro grafo $\mathbb{G}^{\prime}$, que inicialmente é uma cópia de $\mathbb{G}$. Durante o algoritmo será necessário remover alguns vértices e arestas. Logo, iremos remover do grafo $\mathbb{G}^{\prime}$. Implicando que durante todo o algoritmo o grafo $\mathbb{G}$ permanece inalterado e como só iremos remover vértice e arestas de $\mathbb{G}^{\prime}$, temos que 
em qualquer instante $\mathbb{G}^{\prime}$ é um subgrafo de $\mathbb{G}$.

Além dos dois grafos, os procedimentos para construir um grafo dos caminhos dirigidos utilizam muitas variáveis. Estas variáveis serão dividas em quatro categorias:

A. Variáveis associadas a cada vértice do grafo de entrada $\mathbb{G}$;

B. Variáveis associadas ao grafo dos caminhos dirigidos $\mathbb{D}$;

C. Variáveis associadas ao grafo de entrada $\mathbb{G}$ (informações gerais);

D. Variáveis gerais.

Para facilitar o entendimento da semântica de cada variável, considere a figura A.1 que apresenta uma orientação das arestas da borda externa do grafo plano triangulado.

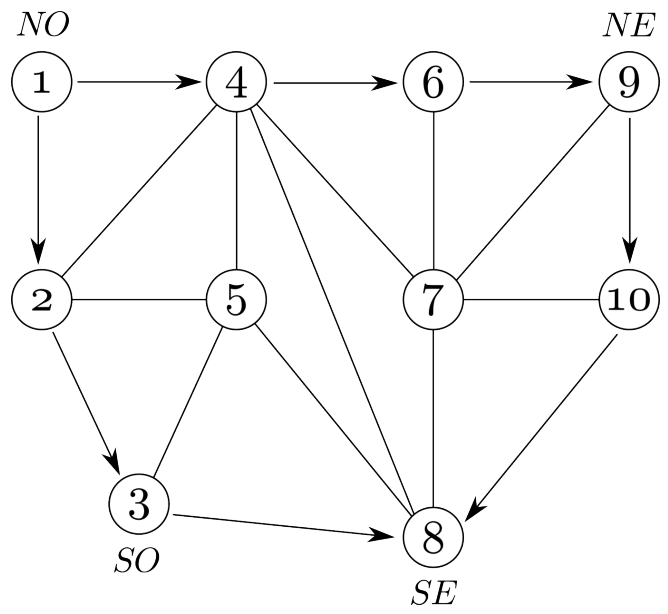

Figura A.1: Orientação das arestas da borda do grafo plano triangulado.

Categoria A: Variáveis associadas a cada vértice do grafo de entrada $\mathbb{G}$.

PertenceGCD é uma variável booleana que inicialmente é falsa para todos os vértices de $\mathbb{G}$. Quando um vértice $v$ é adicionado a $\mathbb{D}$, a variável Pertence $G C D$ de $v$ deve possuir valor verdadeiro;

BordaDireita é uma variável booleana que é verdadeira se e só se o vértice pertence ao caminho direito da borda do subgrafo que está sendo processado. Durante o processamento do algoritmo, alguns vértices irão modificar o valor de falso para verdadeiro. Entretanto, uma vez que seu valor for verdadeiro, como ele não será processado novamente, ele nunca será modificado para falso. Para o exemplo da figura A.1, BordaDireita é verdadeiro para os vértices 8, 9 e 10;

BordaSuperior é uma variável booleana de comportamento similar à variável BordaDireita, exceto pelo fato que possui valor verdadeiro se e só se o vértice pertence 
ao caminho superior da borda do subgrafo que está sendo processado. Para o exemplo da figura A.1, BordaSuperior é verdadeiro para os vértices 1, 4, 6 e 9;

BordaInferior é uma variável booleana de comportamento similar à variável BordaDireita, exceto pelo fato que possui valor verdadeiro se e só se o vértice pertence ao caminho inferior da borda do subgrafo que está sendo processado. Para o exemplo da figura A.1, BordaDireita é verdadeiro para os vértices 3 e 8;

ProxSuperior é uma variável que serve para implementar uma lista encadeada dos vértices do caminho superior da borda. Os vértices do caminho superior da borda serão armazenados da esquerda para direita. Esta variável só será utilizada se o vértice em questão pertence ao caminho superior da borda. Caso pertença, ProxSuperior referencia o próximo vértice do caminho superior da borda. Na figura A.1, ProxSuperior é utilizada para encadear, inicialmente, os vértices 1, 4, 6 e 9 (nesta ordem);

ProxInferior é uma variável similar a ProxSuperior, entretanto, ela irá encadear o caminho inferior da borda do subgrafo que está sendo processado. Na figura A.1, ProxInferior é utilizada para encadear, inicialmente, os vértices 3 e 8;

BordaEsquerda é uma variável que pode possuir um dos três valores: 0,1 ou 2. Seja $v \in$ $V(\mathbb{G})$, temos que $v \cdot$ BordaEsquerda $=2$ se e somente se $v$ nunca pertenceu ao caminho esquerdo da borda de algum subgrafo de $\mathbb{G}$. Caso contrário, BordaEsquerda pode ser igual a 0 ou 1. Como será apresentado com mais detalhes futuramente, o algoritmo para construir o grafo $\mathbb{D}$ irá percorrer o caminho esquerdo da borda de $\mathbb{G}$, depois irá remover este caminho e processar o novo caminho esquerdo da borda do subgrafo resultante, e assim sucessivamente. O novo caminho esquerdo da borda é construído enquanto percorremos o caminho esquerdo atual. Para distinguir os vértices dos dois caminhos, vamos utilizar os valores 0 e 1 para rotular os vértices destes caminhos. Por exemplo, se os vértices do caminho esquerdo atual possuem BordaEsquerda $=0$ (BordaEsquerda $=1$ ) então os vértices do próximo caminho esquerdo devem possuir BordaEsquerda $=1$ (BordaEsquerda $=0)$;

ChegouBordaDireita é uma variável que possui valor inicial NULO para todos os vértices de $\mathbb{G}$. Durante o processamento do algoritmo, esta variável irá armazenar o fato de que o vértice pode ser adjacente a certos vértices do caminho direito da borda. Como será mostrado mais tarde, esta variável garante que a complexidade do algoritmo é $O(n)$, onde $n$ é o número de vértices de $\mathbb{G}$;

ProxEsq é uma variável similar a ProxSuperior, entretanto, ela irá encadear o caminho esquerdo da borda do subgrafo que está sendo processado. Na figura A.1, ProxEsq é utilizada para encadear, inicialmente, os vértices 1,2 e 3.

Categoria B: Variáveis associadas ao grafo dos caminhos dirigidos $\mathbb{D}$. 
VerticeInicial é um vértice pertencente a $\mathbb{D}$ de modo que quando o subgrafo de $\mathbb{G}$ está sendo processado, todos os caminhos que serão adicionados a $\mathbb{D}$ devem iniciar em VerticeInicial;

VerticeFinal é um vértice pertencente a $\mathbb{D}$ de modo que quando o subgrafo de $\mathbb{G}$ está sendo processado, todos os caminhos que serão adicionados a $\mathbb{D}$ devem terminar em VerticeFinal;

Terminou é uma variável booleana que possui valor falso enquanto existir algum vértice do subgrafo que está sendo processado que ainda não foi adicionado a $\mathbb{D}$;

Pai é um vértice pertencente a $\mathbb{D}$ de modo que o próximo vértice adicionado a $\mathbb{D}$ deve ser filho do vértice Pai.

Categoria C: Variáveis associadas ao grafo de entrada $\mathbb{G}$ (informações gerais).

$\boldsymbol{N O}$ é uma variável que referencia um vértice do canto superior esquerdo do subgrafo que está sendo processado. Por exemplo, na figura A.1, temos que $N O=1$;

$\boldsymbol{N E}$ é uma variável que referencia um vértice do canto superior direito do subgrafo que está sendo processado. Por exemplo, na figura A.1, temos que $N E=9$;

$\boldsymbol{S O}$ é uma variável que referencia um vértice do canto inferior esquerdo do subgrafo que está sendo processado. Por exemplo, na figura A.1, temos que $S O=3$;

$\boldsymbol{N E}$ é uma variável que referencia um vértice do canto inferior direito do subgrafo que está sendo processado. Por exemplo, na figura A.1, temos que $S E=8$;

$\boldsymbol{N} \boldsymbol{O}_{\text {prox }}$ é o vértice que será o vértice $N O$ após o processamento e remoção do atual caminho esquerdo da borda. Na figura A.1, temos que $N O_{\text {prox }}=4$ quando $N O=1$;

$\boldsymbol{S O}_{\text {prox }}$ é o vértice que será o vértice $S O$ após o processamento e remoção do atual caminho esquerdo da borda. Na figura A.1, temos que $S O_{\text {prox }}=8$ quando $S O=3$;

Categoria D: Variáveis gerais.

Existem muitas variáveis nesta categoria, entretanto, a maior parte delas é fácil identificar qual é a finalidade de seu uso. Iremos apresentar as variáveis mais importantes.

BordaEsqAtual esta variável pode possuir somente valor 0 ou 1. O valor de BordaEsqAtual deve ser igual ao valor da variável BordaEsquerda de todos os vértices que pertencem ao atual caminho esquerdo da borda; 
p variável utilizada para percorrer os vértices do caminho esquerdo da borda do subgrafo que está sendo processado. Note que o caminho inicia pelo vértice $N O$ e termina no vértice $S O$;

$\boldsymbol{p r e d}_{\boldsymbol{p}}$ indica que é o predecessor de $p$ no caminho esquerdo da borda. Quando $p=N O$, temos que $\operatorname{pred}_{p}$ possui valor NULO;

q é uma variável utilizada para construir o próximo caminho esquerdo da borda. Note que este caminho irá se iniciar no vértice $N O_{\text {prox }}$ e irá terminar no vértice $S O_{\text {prox }}$.

Iremos utiliza a notação $a . b$ para simbolizar a variável $b$ associada a variável $a$.

\subsection{Algoritmo}

Vamos apresentar nesta seção o algoritmo para construir um grafo de caminhos dirigidos de um grafo plano triangulado. Este algoritmo possui muitos casos especiais a serem tratados. Vamos dividir o algoritmo em procedimentos, de modo que facilite o tratamento dos casos especiais.

A maioria das funções que serão apresentadas a seguir irão modificar os grafos $\mathbb{G}^{\prime}$ e $\mathbb{D}$. Assim, iremos considerar que os grafos são variáveis globais. Ainda, vamos abusar da notação dos algoritmos de modo que as funções que modifiquem um deste grafos irá possuir como saída qual grafo será modificado na função, entretanto, não será devolvido algum valor. Para simplificar o entendimento, iremos relacionar na entrada de cada algoritmo todas as variáveis necessárias para seu funcionamento, incluindo as variáveis globais.

A função CONSTRUIR-GCD consiste em inicializar algumas variáveis e chama a função principal: PLACE.

\section{Algoritmo Construir-GCD ( $\mathbb{G})$}

EnTRADA: Grafo plano triangulado $\mathbb{G}$.

SAÍDA: Grafo de caminhos dirigidos $\mathbb{D}$ de $\mathbb{G}$.

1. Escolha os vértices $N O, N E, S O$ e $S E$ conforme apresentado no algoritmo KOŹMIŃSKI E KINNEN na seção 4.4;

2. Inicialize as listas encadeadas dos caminhos:

(a) caminho esquerdo da borda de $N O$ a $S O$ utilizando ProxEsq;

(b) caminho superior da borda de $N O$ a $N E$ utilizando ProxSuperior;

(c) caminho inferior da borda de $S O$ a $S E$ utilizando ProxInferior;

3. Inicialize BordaEsquerda com 0 para todos os vértices do caminho esquerdo da borda

e BordaEsquerda com 2 para todos os outros vértices de $\mathbb{G}$;

4. Inicialize BordaSuperior como verdadeiro para todos os vértices do caminho superior 
da borda. Para todos os outros vértices de $\mathbb{G}$, BordaSuperior deve ser falso;

5. Inicialize Bordalnferior como verdadeiro para todos os vértices do caminho inferior da borda. Para todos os outros vértices de $\mathbb{G}$, Bordalnferior deve ser falso;

6. Inicialize BordaDireita como verdadeiro para todos os vértices do caminho de $N E$ até $S E$ (inclusive). Para todos os outros vértices de $\mathbb{G}$, BordaDireita deve ser falso;

7. Inicialize ChegouBordaDireita com NULO para cada vértice de $\mathbb{G}$;

8. Inicialize $\mathbb{D}$ de modo que $V(\mathbb{D})=v_{\infty}$ e $E(\mathbb{D})=\emptyset$;

9. $\quad \mathbb{G}^{\prime} \leftarrow \mathbb{G}$;

10. PAThs $\left(N O, N E, S O, S E, v_{\infty}, N U L O, 0\right)$;

É fácil ver que as linhas 1-9 da função CONSTRUIR-GCD podem ser executados em tempo linear. Antes de apresentar a função PATHs, vamos introduzir algumas rotinas necessárias.

Algoritmo Próximos-NO-SO $(N O, S O)$

ENTRADA: Grafo $\mathbb{G}^{\prime}$ e variáveis $N O$ e $S O$.

SAÍDA: Grafo $\mathbb{G}^{\prime}$ e variáveis $N O_{\text {prox }}$ e $S O_{\text {prox }}$.

1. Se NO.ProxSuperior $=N U L O$ então

2. $\quad N O_{\text {prox }} \leftarrow N O$

3. Senão

4. $\quad N O_{\text {prox }} \leftarrow$ NO.ProxSuperior;

5. $\quad \mathbb{G}^{\prime} \leftarrow \mathbb{G}^{\prime}-\{N O\}$

6. Se SO.ProxInferior $=N U L O$ então

7. $S O_{\text {prox }} \leftarrow S O$;

8. Senão

9. $S O_{\text {prox }} \leftarrow$ SO.ProxInferior;

10. $\mathbb{G}^{\prime} \leftarrow \mathbb{G}^{\prime}-\{S O\}$;

11. Devolva $N O_{\text {prox }}, S O_{\text {prox }}$;

A função PRóximos-NO-SO identifica quais vértices as variáveis $S O_{\text {prox }}$ e $N O_{\text {prox }}$ devem referenciar. Caso $N O$ for o último vértice do caminho superior da borda do grafo que está sendo processado, então $N O_{\text {prox }}$ deve ser igual a $N O$. De modo análogo, caso $S O$ for o último vértice do caminho inferior da borda, então $S O_{\text {prox }}$ deve ser igual a $S O$. Se o vértice $N O$ não for o último vértice do caminho superior, então removemos $N O$ de $\mathbb{G}^{\prime}$. De modo análogo para $S O$. 


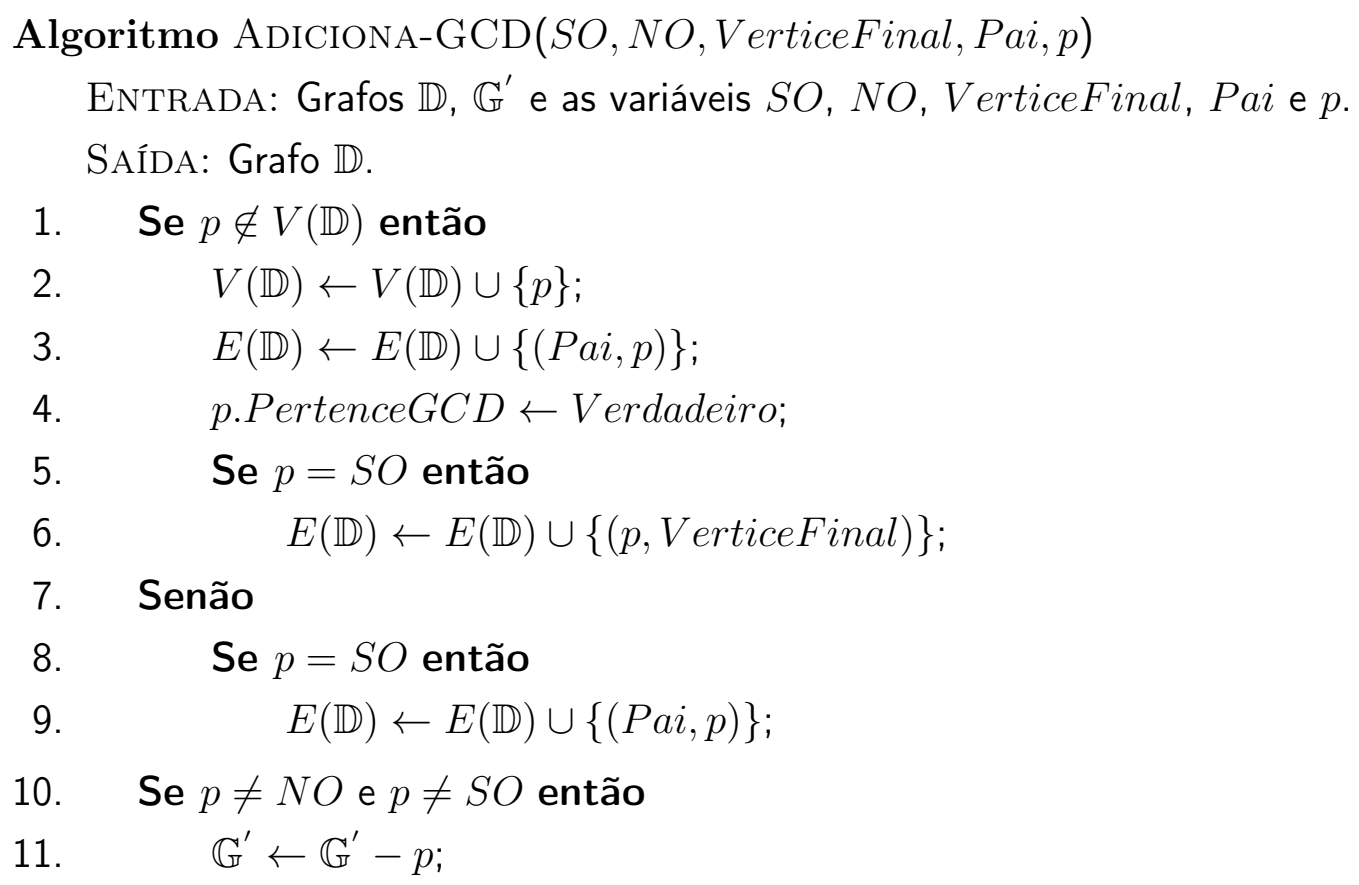

A função Adiciona-GCD adiciona o vértice $p$ a $\mathbb{D}$, caso ele já exista em $\mathbb{D}$, deve ser adicionado somente uma aresta. Caso $p \notin V(\mathbb{D})$, então adiciona $p$ como filho do vértice Pai. Ainda, se $p$ é o último vértice do caminho esquerdo da borda do subgrafo que está sendo processado, então $p$ deve ser o último vértice do caminho dirigido que está sendo adicionado a $\mathbb{D}$. Entretanto, como todos caminhos devem terminar no vértice VerticeFinal, então adicionamos a aresta $(p$, VerticeFinal $)$ a $\mathbb{D}$. Caso $p \in V(\mathbb{D})$, temos duas possibilidades, ou $p=N O$ ou $p=S O$. Isso decorre do fato que os vértice $N O$ e $S O$ do subgrafo que está sendo processado atualmente podem pertencer a diversos caminhos esquerdos da borda. Caso $p=N O$, não precisamos fazer nada, pois a aresta (VerticeInicial, $p$ ) já deve ter sido adicionada anteriormente a $\mathbb{D}$. Caso contrário, quando $p=S O$, devemos adicionar somente a $\operatorname{aresta}(P a i, p)$ a $\mathbb{D}$. É fácil ver que o vértice Pai deve ter sido adicionado enquanto o atual caminho esquerdo da borda estava sendo processado, pois este caminho deve conter ao menos um vértice que não tinha sido processado anteriormente. Ainda, como $p=S O$, em algum momento anterior, a aresta ( $p$,VerticeFinal) foi adicionada a $\mathbb{D}$.

Ainda, a função ADICIONA-GCD remove de $\mathbb{G}^{\prime}$ todos os vértices intermediários do caminho esquerdo da borda. 
Algoritmo Paths(NO, NE, SO, SE, VerticeInicial, VerticeFinal, BordaEsqAtual) EntradA: Grafo $\mathbb{D}$ e variáveis $N O, N E, S O, S E$, VerticeInicial, VerticeFinal, BordaEsqAtual.

SAÍDA: Grafo $\mathbb{D}$ e grafo $\mathbb{G}^{\prime}$.

/* Cobre todos os vértices do subgrafo de $\mathbb{G}$ limitado pelos vértices $N O, N E, S O$ e $S E$ utilizando caminhos direcionados. Todos estes caminhos serão adicionados em $\mathbb{D}$ iniciando no vértice VerticeInicial e terminando no vértice VerticeFinal. * /

1. /* Obtém a cobertura dos vértices utilizando caminhos */

2. Repita

3. Pai $\leftarrow$ VerticeInicial;

4. $\quad$ Terminou $\leftarrow$ Verdadeiro;

5. $\quad N O_{\text {prox }}, S O_{\text {prox }} \leftarrow$ PRÓximOS-NO-SO $(N O, S O)$;

6. $\quad p \leftarrow N O ; / *$ Vértice do atual caminho esquerdo da borda */

7. $\operatorname{pred}_{p} \leftarrow N U L O ; / *$ Predecessor de $p$ no atual caminho esquerdo da borda */

8. $\quad q \leftarrow N O_{\text {prox }} ; *$ Primeiro vértice do próximo caminho esquerdo da borda */

9. $\quad$. BordaEsquerda $\leftarrow 1-$ BordaEsqAtual;

$10 \quad$ /* Percorre o atual caminho esquerdo da borda */

11. Repita

12. Adiciona-GCD (SO, NO, VerticeFinal, Pai, $p)$;

13. Pai $\leftarrow p$;

14. Se $\quad$ S.BordaDireita então

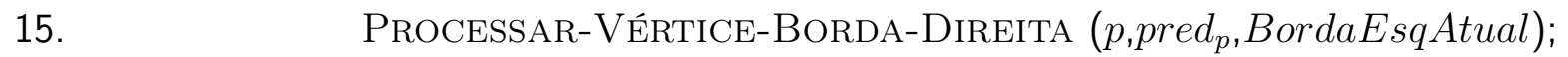

16. Senão

17. Processar-VÉrtice-NÃo-Borda-Direita $(p, q$, BordaEsqAtual);

18. $\operatorname{pred}_{p} \leftarrow p$;

19. $\quad p \leftarrow$ p.ProxEsq;/* Avança $p * /$

20. Até $(p=N U L O)$;

21. $N O \leftarrow N O_{\text {prox }} ; S O \leftarrow S O_{\text {prox }}$;

22. BordaEsqAtual $\leftarrow 1-$ BordaEsqAtual;

23. Até $($ Terminou $=$ Verdadeiro);

A função PATHS encontra uma cobertura por caminhos dos vértices do subgrafo limitado pelos vértices $N O, N E, S O$ e $S E$. Todos os caminhos serão adicionados a $\mathbb{D}$ de modo que se iniciem no VerticeInicial e terminem no VerticeFinal. Embora não é possível visualizar com o que foi apresentado até o momento, a função PATHS é recursiva, pois as funções Processar-Vértice-Borda-Direita e Processar-VÉrtice-Não-BordaDiReita invocam a função PATHS. 
É fácil ver que o laço das linhas 2-23 objetiva percorrer todos os caminhos esquerdos. Em uma iteração, será percorrido o caminho esquerdo da borda e já é construído o próximo caminho esquerdo. Este laço termina quando todos os vértices do subgrafo forem adicionados a $\mathbb{D}$. Para que todos os caminhos que irão cobrir os vértices do subgrafo iniciarem com VerticeInicial, a variável Pai recebe o valor VerticeInicial na linha 3. A variável Terminou recebe o valor verdadeiro na linha 4 e garante que quando todos os vértice do subgrafo forem adicionados a $\mathbb{D}$ o algoritmo para. As variáveis $p$, pred $_{p}$ e $q$ são inicializadas nas linhas 69. Estas variáveis irão percorrer o atual caminho esquerdo da borda e irão construir, caso exista, o próximo caminho esquerdo da borda.

O laço das linhas 11-20 é responsável por percorrer o atual caminho esquerdo da borda, onde $p$ é o vértice que está sendo processando. Este vértice é adicionado a $\mathbb{D}$ na linha $12 \mathrm{e}$ nas linhas 14-17 ele é processado. Nas linhas 18 e 19 avançamos $p$ e $\operatorname{pred}_{p}$. O algoritmo para quando $p$ é NULO.

Após processar o caminho esquerdo da borda, as linhas 21 e 22 modificam o subgrafo que deverá ser processado atualizando os valores das variáveis NO,SO e BordaEsqAtual.

A função PATHS é bem simples, entretanto, conforme já dito anteriormente, para construir um grafo de caminhos dirigidos existem alguns casos especiais que devem ser tratados. Antes de apresentar as funções PROCESSAR-VÉRTICE-BordA-Direita e Processar-VÉRTICE-NÃO-BordA-DireitA, vamos apresentar as funções para tratar os casos especiais.

Existem 5 casos especiais a serem tratados. Ilustraremos como tratar quatro destes casos utilizando funções (Processar-Corda, Processar-Canto-Inferior-Esquerdo, Processar-Canto-Superior-Esquerdo e Processar-Componente-Pendente) e o quinto caso será tratado utilizando a variável ChegouBordaDireita que está associada a cada vértice de $\mathbb{G}$. Essencialmente, para cada caso iremos isolar a região do grafo que viola o lema 5.2 e aplicar a função PLACE neste subgrafo. Após cobrir os vértices do subgrafo utilizando caminhos direcionados, iremos definir o próximo caminho esquerdo da borda e prosseguimos com o processamento.

O primeiro caso especial é quando uma corda é detectada. A função PROCESSAR-CordA soluciona este caso. 
Algoritmo ProcessAR-CoRdA(ch_p,ch_r, BordaEsqAtual)

EntradA: Grafos $\mathbb{D}, \mathbb{G}^{\prime}, \mathbb{G}$ e variáveis $c h \_p, c h \_r$ e BordaEsqAtual.

SAÍDA: Grafos $\mathbb{D}$ e $\mathbb{G}^{\prime}$.

1. $\quad c h \_S E \leftarrow c h \_p$;

2. Enquanto $c h \_S E . P r o x E s q \neq c h \_r$ faça

3. $\quad c h \_S E \leftarrow$ Primeiro vértice da lista de adjacência de $c h \_r$ após $c h \_S E$ no sentido anti-horário em $\mathbb{G}^{\prime}$;

4. $\quad c h \_f i m \leftarrow$ Primeiro vértice da lista de adjacência de $c h \_S E$ após $c h \_r$ no sentido horário em $\mathbb{G}$;

5. $u \leftarrow c h \_S E$;

6. Enquanto u.BordaEsquerda = BordaEsqAtual e u.Pertence $G C D=$ Falso faça

7. $\quad c h \_S O \leftarrow u$;

8. $u \leftarrow$ Primeiro vértice da lista de adjacência de $c h \_$fim após $u$ no sentido anti-horário em $\mathbb{G}$;

9. $\quad$ prox $\leftarrow$ ch_p;

10. Repita

11. $u \leftarrow$ prox;

12. $\quad$ u.BordaDireita $\leftarrow$ Verdadeiro;

13. $\operatorname{prox} \leftarrow$ Primeiro vértice da lista de adjacência de $c h \_r$ após prox no sentido anti-horário em $\mathbb{G}^{\prime}$;

14. $\mathbb{G}^{\prime} \leftarrow \mathbb{G}^{\prime}-\left\{\left(u, c h \_r\right)\right\}$;

15. Até $\left(u=c h \_S E\right)$;

16. prox $\leftarrow$ cp_SE;

17. ant $\leftarrow N U L O$;

18. Repita

19. $u \leftarrow$ prox;

20. u.ProxInferior $\leftarrow$ ant;

21. u.BordaInferior $\leftarrow$ Verdadeiro;

22. $\quad$ u.BordaEsquerda $\leftarrow 2$;

23. $\quad$ ant $\leftarrow$ Referência para $u$;

24. $\quad$ prox $\leftarrow$ Primeiro vértice da lista de adjacência de $c p \_$fim após prox no sentido anti-horário em $\mathbb{G}^{\prime}$;

25. Até $\left(u=c p_{-} S O\right)$;

26. ch_SO.ProxEsq $\leftarrow N U L O$;

27. PATHS (ch_p,ch_p, ch_SO,ch_SE, ch_p, ch_fim, BordaEsqAtual);

De modo análogo a seção anterior, considere as figuras A.2A e A.2B. A aresta $\left(c h \_p, c h \_r\right)$ é uma corda de $\mathbb{G}$ de modo que $c h \_p$ é predecessor de $c h \_r$ no caminho 
esquerdo da borda do subgrafo que está sendo processado. No momento que a função PROCESSAR-CORDA for chamada para tratar este caso, o vértice $c h \_p$ pertence a $\mathbb{D}$ enquanto o vértice $c h \_r$ não pertence a $\mathbb{D}$.

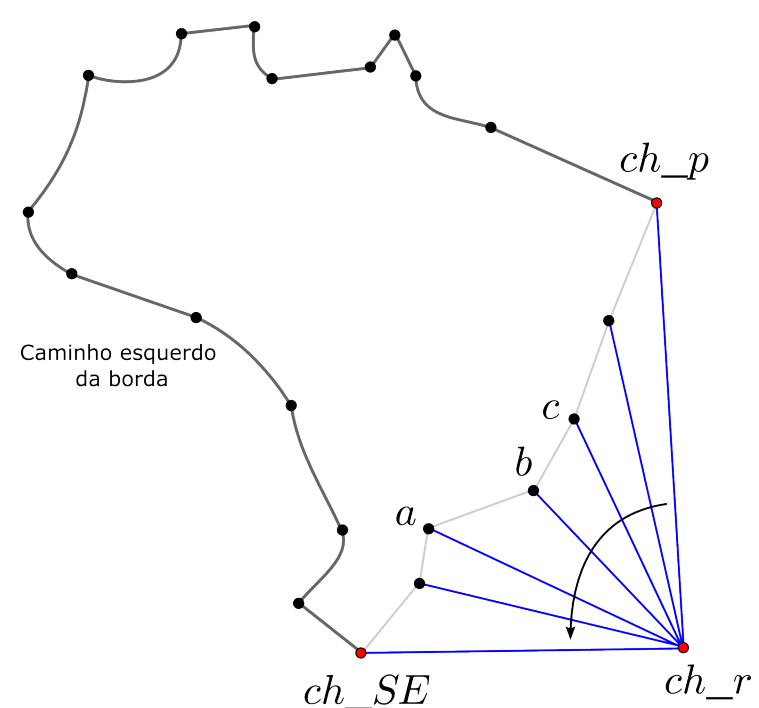

(A)

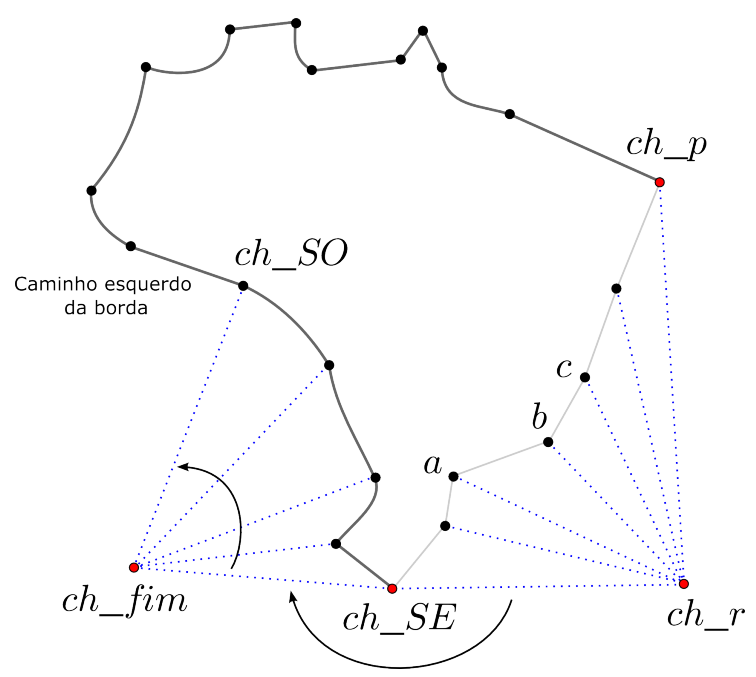

(B)

Figura A.2: Ilustração de uma corda (ch_p,ch_r). [BS88]

Inicialmente, devemos isolar o subgrafo II. As linhas 1-3 do algoritmo PROCESSAR-CORDA encontram o vértice $c h \_S E$ percorrendo a lista de adjacência do vértice $c h \_r$ no sentido anti-horário começando pelo vértice $c h \_p$. O vértice $c h \_S E$ é o predecessor de $c h \_r$ no atual caminho esquerdo da borda. A região interna ao circuito $C$ (I) é isolada do restante de $\mathbb{G}^{\prime}$ nas linhas 9-15.

Para cobrir todos os vértices do subgrafo utilizando caminhos, precisamos identificar os vértices que são os cantos $N O, N E, S O$ e $S E$. Assim que identificados todos eles, podemos aplicar a função PATHS.

O primeiro vértice da lista de adjacência de ch_SE no sentido horário após o vértice ch_r, será chamado de ch_fim (linha 4).

Agora, vamos percorrer a lista de adjacência de $c h \_$fim no sentido anti-horário iniciando no vértice $c h \_S E$ e terminando no vértice $c h \_S O$, onde o vértice $c h$ _SO é o último vértice que pertence ao caminho esquerdo e não pertence a $\mathbb{D}$ (linhas 5-8).

O grafo resultante (figura A.2B) está isolado em $\mathbb{G}^{\prime}$. O vértice $c h \_p$ será o canto superior esquerdo e o canto superior direito. O vértice $c h \_S O$ será o canto inferior esquerdo e o vértice $c h \_S E$ será o canto inferior direito. Entretanto, antes de aplicar a função PATHS no grafo resultante, precisamos inicializar os caminhos esquerdo, superior, direito e inferior do subgrafo isolado (linhas 16-26). Para todos os vértices entre ch_SO e ch_SE que foram encontrados no laço das linhas 12-15, as linhas 16-25 atualizam a variável ProxInferior para representar o caminho dirigido de $c h \_S O$ a $c h \_S E$. Ainda, para cada vértice do caminho, 
atualizam BordaInferior para verdadeiro e BordaEsquerda para 2.

Finalmente, após a execução de PATHs, todos os vértices do subgrafo foram adicionados a $\mathbb{D}$ e como todos os caminhos que cobrem estes vértices iniciam em $c h \_p$ e terminam em $c h \_f i m$, garantimos que $c h \_p$ não é um ancestral distante de $c h \_r$, pois não existirá um caminho de comprimento maior ou igual a 2 entre os vértices $c h \_p$ e $c h \_r$ em $\mathbb{D}$.

O segundo caso especial é quando uma aresta $(p, r)$ com as seguintes propriedades pertence a $\mathbb{D}$ :

P1. $p$ pertence ao atual caminho esquerdo da borda e $p \neq S O$;

P2. $r$ pertence ao atual caminho inferior da borda e $r \neq S O$.

Este caso é ilustrado na figura A.3. Esta situação surge quando estamos construindo o próximo caminho esquerdo e existe uma aresta entre dois vértices, um do caminho esquerdo da borda e outro do caminho inferior da borda. Assim, precisamos tratar como o canto inferior esquerdo delimitado por esta aresta será processado. A função Processar-CANTOINFERIOR-ESQUERDO faz um processamento especial para esta região crítica.

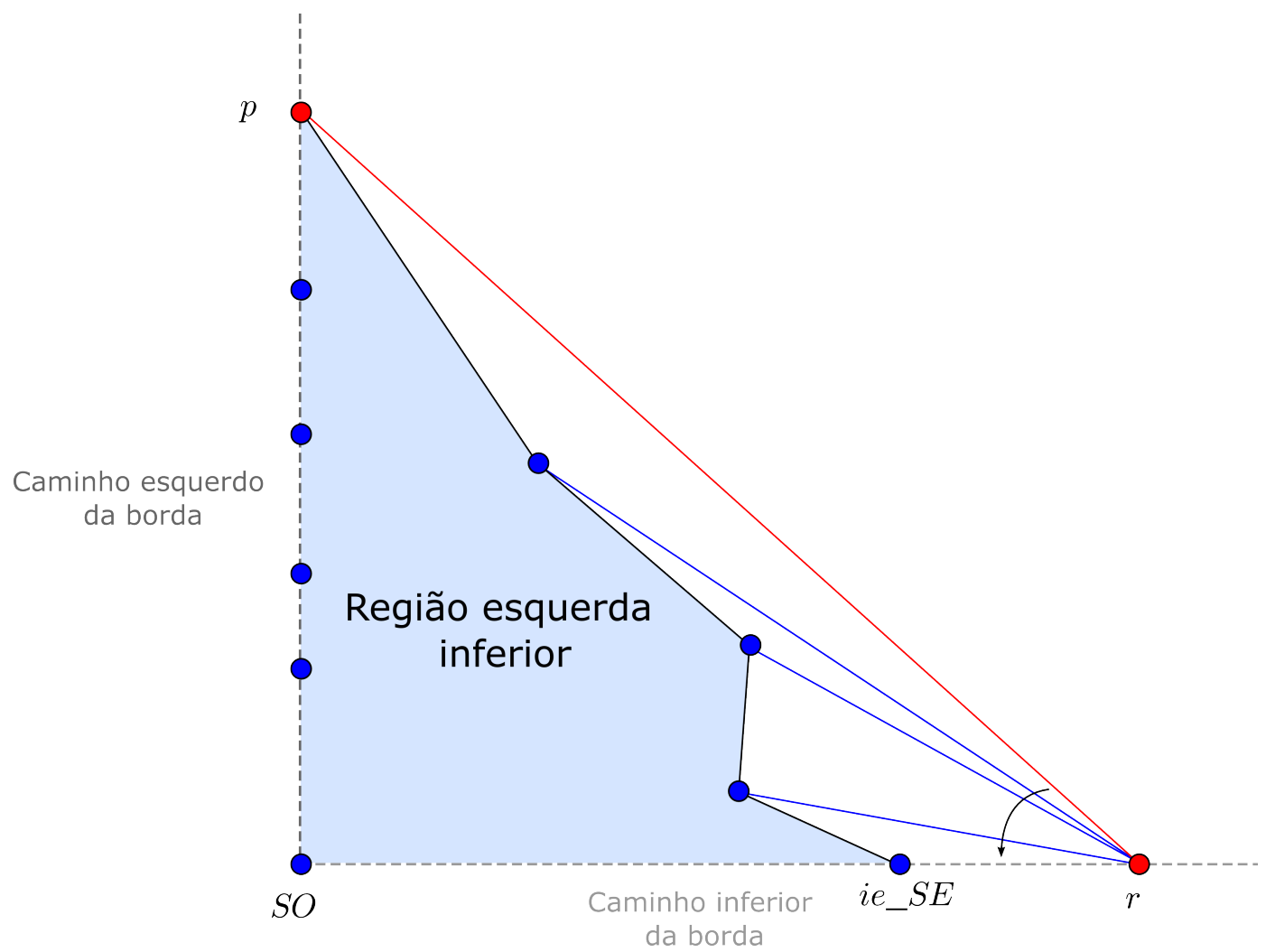

Figura A.3: Ilustração da região esquerda inferior (segundo caso especial). [BS88] 


\section{Algoritmo Processar-Canto-Inferior-Esquerdo $(p, r)$}

Entrada: Grafos $\mathbb{D}, \mathbb{G}^{\prime}$ e vértices $p, r \in V\left(\mathbb{G}^{\prime}\right)$.

SAÍDA: Grafos $\mathbb{D}$ e $\mathbb{G}^{\prime}$.

1. $i e \_S E \leftarrow p$;

2. Enquanto ie_SE.BordaInferior $=$ Falso faça

3. $\quad i e_{-} S E \leftarrow$ Primeiro vértice da lista de adjacência de $r$ após $i e \_S E$ no sentido anti-horário em $\mathbb{G}^{\prime}$;

4. $\quad \operatorname{prox} \leftarrow p$;

5. Repita

6. $u \leftarrow$ prox;

7. u.BordaDireita $\leftarrow$ Verdadeiro;

8. $\operatorname{prox} \leftarrow$ Primeiro vértice da lista de adjacência de $r$ após $u$ no sentido anti-horário em $\mathbb{G}^{\prime}$;

9. $\mathbb{G}^{\prime} \leftarrow \mathbb{G}^{\prime}-\{(r, u)\}$;

10. Até $\left(u=i e_{-} S E\right)$;

11. PATHS ( $p, p, S O, i e \_S E, p$, VerticeFinal, BordaEsqAtual);

Igualmente ao caso anterior, precisamos isolar o subgrafo que iremos processar. Para isso, percorremos a lista de adjacência do vértice $r$ no sentido anti-horário iniciando no vértice $p$ e terminando no primeiro vértice que pertence ao atual caminho inferior da borda, digamos que $i e_{-} S E$. As linhas 1-3 identificam o vértice $i e_{-} S E$, enquanto as linhas 4-10 isolam a região que precisamos tratar. Precisamos mostrar que o subgrafo $\mathbb{G}^{\prime}$ que está sendo processado satisfaz o seguinte lema:

Lema A.1: Seja $\mathbb{G}^{\prime}$ um grafo que está sendo processado pelo algoritmo. $\mathbb{G}^{\prime}$ não contém uma aresta $u v$ de modo que $u$ e $v$ pertençam ao caminho inferior da borda $e$ $u$ e $v$ não são adjacentes no caminho inferior da borda.

Demonstração: Vamos provar este resultado analisando as funções que modificam o grafo $\mathbb{G}^{\prime}$.

É fácil ver que inicialmente o grafo $\mathbb{G}^{\prime}$ satisfaz a tese, pois como $\mathbb{G}^{\prime}=\mathbb{G}$, temos que os vértices $N O, S O, S E$ e $S O$ foram escolhidos de modo que o caminho inferior da borda não possua uma corda que satisfaz as propriedades P1, P2 e P3.

Vamos mostrar que as chamadas da função PATHs preservam a invariante. A seguir, vamos examinar cada uma das chamadas:

a) Linha 27 da função Processar-Corda.

Podemos ver na figura A.2B que o caminho inferior da borda é composto pelo segmento do atual caminho esquerdo da borda entre os vértices $c h \_S O$ e $c h \_S E$. Todos estes 
vértices são adjacentes ao vértice $c h \_f i m$. Suponha que existe uma aresta $u v$ que viola a tese, logo, o triângulo formado pelos vértice $u, v$ e $c h \_$fim é um triângulo que não é face, um absurdo. Logo, não existe tal aresta.

b) Linha 11 da função Processar-Canto-Inferior-Esquerdo.

O caminho inferior da borda do subgrafo que será processado por PATHS é um segmento esquerdo do atual caminho inferior da borda. Logo, se a tese era válida para o caminho inferior da borda, então a tese é válida para o próximo caminho inferior da borda.

c) Linha 28 da função Processar-CAnto-Superior-Esquerdo.

Como todos os vértices do novo caminho inferior da borda é adjacente ao vértice $p$, temos pelo mesmo argumento de (a) que a tese deve ser válida.

d) Linha 44 da função Processar-Componente-Pendente.

De modo análogo aos casos (a) e (c), o vértice $c p \_p$ é adjacente a todos os vértices do novo caminho inferior da borda. Logo, pelo mesmo argumento, não existe tal aresta.

Portanto, temos que a tese é válida.

O resultado do lema A.1 implica que $i e \_S E$ e $r$ devem ser adjacentes no caminho inferior da borda. Ainda, ie_SE.ProxInferior $=r$ (como ilustrado na figura A.3). Considere todos os vértices visitados quando percorremos a lista de adjacência do vértice $r$ no sentido antihorário inciando por $p$ e terminando no vértice $i e_{\_} S E$. Estes vértices (incluindo $p$ e $i e_{-} S E$ ) serão o caminho direito da borda da região que será isolada. Ainda, devemos remover todos as arestas entre $r$ e os vértices do caminho direito para isolar a região que precisamos tratar do resto do grafo.

Os vértices da região isolada são adicionados a $\mathbb{D}$ através da chamada da função PATHS na linha 11. O vértice $p$ representará o canto superior esquerdo e canto superior direito. Ademais, todos os caminhos que cobrem esta região deverão iniciar no vértice $p$ e terminar no vértice VerticeFinal (passado como parâmetro).

O terceiro caso especial é semelhante ao segundo caso, entretanto, a região que deverá ser tratada está do lado esquerdo superior. Assim, este caso se caracteriza por uma aresta pr que satisfaz:

P1. $p$ pertence ao atual caminho esquerdo da borda;

P2. $r$ pertence ao atual caminho superior da borda e $r \neq N O$; e

P3. A aresta pr não pertence ao caminho superior da borda. 
A figura A.4 ilustra este caso. O segmento azul do atual caminho esquerdo da borda representado na figura A.4 será processado e deletado do grafo $\mathbb{G}^{\prime}$. O caminho em vermelho que passa pelos vértices $N O_{\text {prox }}, s e_{-} S O$, se_SE, se_NE e $r$ fará parte do próximo caminho esquerdo da borda. Assim, quando identificamos uma aresta pr conforme caracterizado anteriormente, precisamos consertar o próximo caminho esquerdo da borda e processar recursivamente a região superior esquerda.

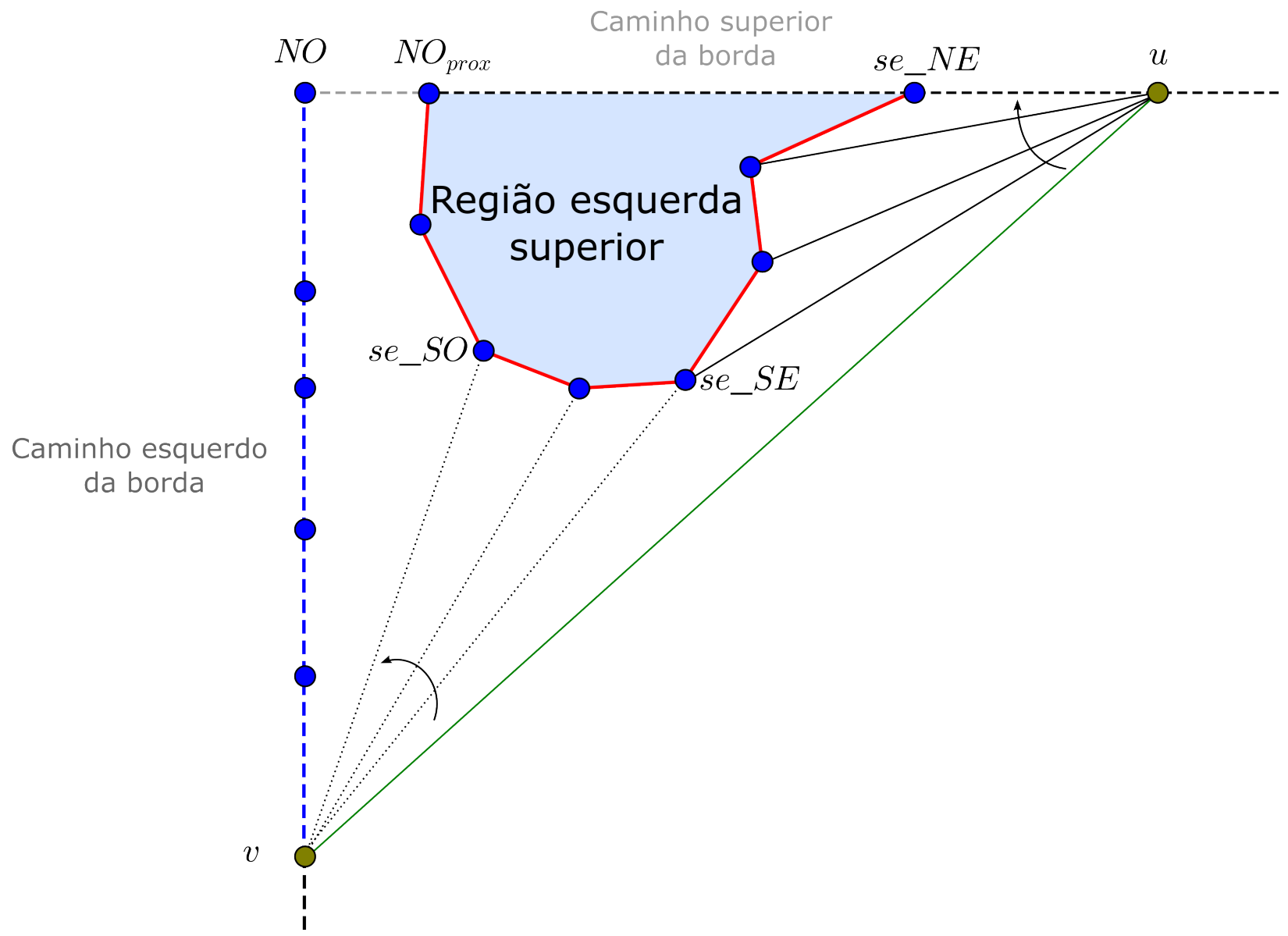

Figura A.4: Ilustração da região esquerda superior (terceiro caso especial). [BS88]

De modo análogo a Processar-CANTO-INFERIOR-Esquerdo, para isolar a região esquerda superior do restante de $\mathbb{G}^{\prime}$, precisamos identificar os vértices se_SO, se_SE e se_NE. Podemos assumir que $r \neq N O_{\text {prox }}$, pois caso $r=N O_{\text {prox }}$, então, conforme veremos adiante, as condições do quarto caso especial (componente pendente) são satisfeitas, onde $c p \_p=p$ e $c p \_r=r$. Logo, podemos tratar este caso utilizando a função ProcessarComponente-Pendente. A função Processar-Canto-Superior-Esquerdo parte desta suposição. 
Algoritmo Processar-Canto-Superior-Esquerdo $(p, r)$

EnTRADA: Grafos $\mathbb{D}, \mathbb{G}^{\prime}$ e vértices $p, r \in V\left(\mathbb{G}^{\prime}\right)$.

SAÍDA: Grafos $\mathbb{D}$ e $\mathbb{G}^{\prime}$.

1. $s e \_N E \leftarrow p$;

2. Enquanto $s e \_N E$.BordaSuperior $=$ Falso faça

3. $s_{-} N E \leftarrow$ Primeiro vértice da lista de adjacência de $r$ após se_NE no sentido horário em $\mathbb{G}^{\prime}$;

4. $\quad$ se_SE $\leftarrow$ Primeiro vértice da lista de adjacência de $r$ após $p$ no sentido horário em $\mathbb{G}^{\prime}$;

5. $u \leftarrow p$

6. Repita

7. $u \leftarrow$ Primeiro vértice da lista de adjacência de $r$ após $u$ no sentido horário em $\mathbb{G}^{\prime}$;

8. u.BordaDireita $\leftarrow$ Verdadeiro;

9. $\quad \mathbb{G}^{\prime} \leftarrow \mathbb{G}^{\prime}-\{(r, u)\}$

10. Até $\left(u=s e_{-} N E\right)$;

11. $s e \_S O \leftarrow s e \_S E$;

12. prox $\leftarrow$ Primeiro vértice da lista de adjacência de $p$ após $s e \_S E$ no sentido anti-horário em $\mathbb{G}^{\prime}$;

13. Enquanto prox.BordaEsquerda $=1$ - BordaEsqAtual e prox.Pertence $G C D=$ Falso faça

14. $\quad$ se_SO $\leftarrow$ prox;

15. prox $\leftarrow$ Primeiro vértice da lista de adjacência de $p$ após prox no sentido anti-horário em $\mathbb{G}^{\prime}$;

16. prox $\leftarrow$ se_SO;

17. Repita

18. $u \leftarrow$ prox;

19. prox $\leftarrow$ Primeiro vértice da lista de adjacência de $p$ após prox no sentido horário em $\mathbb{G}^{\prime}$;

20. Se $u=i e_{-} S E$ então

21. $\quad$ u.ProxInferior $\leftarrow N U L O$;

22. Senão

23. u.ProxInferior $\leftarrow$ Referência para prox;

24. u.BordaInferior $\leftarrow$ Verdadeiro;

25. $\quad$ u.BordaEsquerda $\leftarrow 2$;

26. Até $\left(u=i e \_S E\right)$;

27. Se_SO.ProxEsq $\leftarrow N U L O$;

28. PATHS $\left(N O_{\text {prox }}, s e \_N E, s e \_S O, s e \_S E\right.$, VerticeInicial, $p, 1$-BordaEsqAtual); 
Os vértices $s e \_N E$ e $s e_{-} S E$ são identificados nas linhas 1-4. Note que $s e_{-} N E$ deve ser adjacente ao vértice do caminho superior da borda $r$. De modo semelhante ao lema A.1, podemos enunciar o seguinte lema.

Lema A.2: Seja $\mathbb{G}^{\prime}$ um grafo que está sendo processado pelo algoritmo. $\mathbb{G}^{\prime}$ não contém uma aresta uv de modo que $u$ e $v$ pertençam ao caminho superior da borda e $u$ e $v$ não são adjacentes no caminho superior da borda.

Demonstração: A prova deste lema é semelhante a prova do lema A.1.

As linhas 5-10, isolam a região superior esquerda do resto do grafo e seta o valor da variável BordaDireita do caminho direito do subgrafo que foi isolado. As linhas 11-15 identificam o vértice $s e \_S O$. As linhas 16-26 setam as variáveis dos vértices que pertencem ao caminho inferior do subgrafo que foi isolado. Como o subgrafo que foi isolado é limitado pelos vértices $N O_{\text {prox }}$, se_NE, se_SE e se_SO já foi isolado do restante do grafo que está sendo processado $\left(\mathbb{G}^{\prime}\right)$, então a linha 27 corrige o fato que o campo ProxEsq do vértice se_SO deve ser NULO. A linha 28 faz a chamada recursiva para o subgrafo isolado. Assim, todos os caminhos serão adicionados a $\mathbb{D}$ irão iniciar no vértice VerticeInicial e terminam no vértice $p$ (que já pertence a $\mathbb{D}$ ), logo, podemos tratar o segundo caso de modo que o grafo $\mathbb{D}$ não viole a definição do grafo de caminhos dirigidos.

O último caso especial é quando existe alguns vértices a serem processados entre o atual caminho esquerdo da borda e o próximo caminho esquerdo da borda. Cada conjunto conexo destes vértices será chamado de componente pendente. A figura A.5 ilustra uma componente pendente entre o atual e próximo caminho esquerdo da borda.

Vamos tratar uma componente pendente da mesma forma que tratamos os casos anteriores, ou seja, iremos isolar o subgrafo da componente e aplicar recursivamente a função PATHS. O segmento pontilhado do atual caminho esquerdo da borda será processado enquanto tratamos a componente pendente na função Processar-ComponentePendente.

Quando a função Processar-Componente-Pendente for chamada, os vértice $c p \_p$ e $c p \_r$ serão conhecidos. Ainda, existirá ao menos um vértice na componente pendente e a aresta ( $\left.c p \_i n i, c p \_p\right)$ não é uma aresta do atual caminho esquerdo da borda, caso contrário existirá um triângulo que não é face em $\mathbb{G}$. 


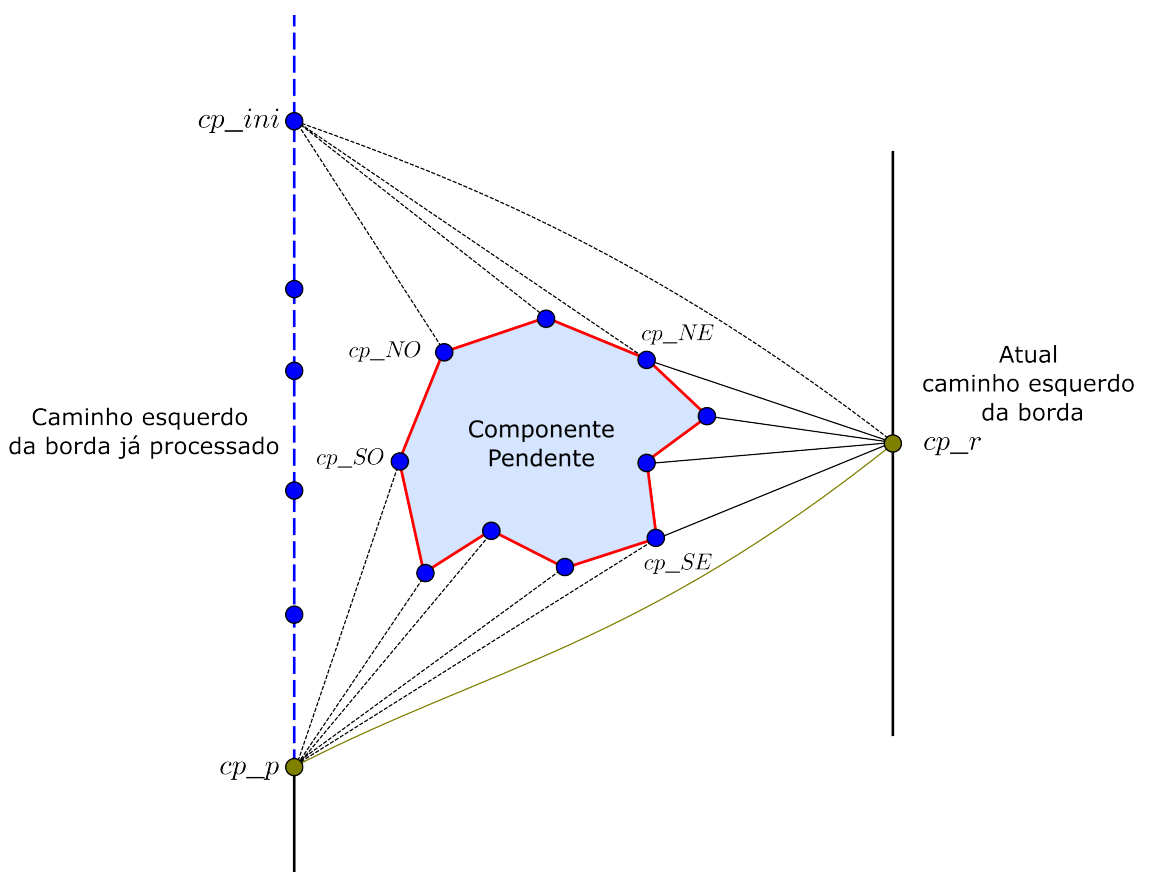

Figura A.5: Componente pendente entre o caminho esquerdo da borda que está sendo processado e o caminho esquerdo da borda que já foi processado. [BS88]

Algoritmo Processar-Componente-Pendente( $c p \_p, c p \_r$, BordaEsqAtual)

ENTRADA: Grafos $\mathbb{D}, \mathbb{G}^{\prime}, \mathbb{G}$ e variáveis $c p \_p, c p \_r$ e BordaEsqAtual.

SAÍDA: Grafo $\mathbb{D}$.

1. $\quad c p \_i n i \leftarrow c p \_p$;

2. Enquanto $c p \_$ini.BordaEsquerda $\neq$BordaEsqAtual faça

3. $\quad c p \_i n i \leftarrow$ Primeiro vértice da lista de adjacência de $c p \_r$ após $c p \_i n i$ no sentido horário em $\mathbb{G}$;

4. $\quad c p \_S E \leftarrow$ Primeiro vértice da lista de adjacência de $c p \_r$ após $c p \_p$ no sentido horário em $\mathbb{G}$;

5. $\quad c p_{-} N E \leftarrow$ Primeiro vértice da lista de adjacência de $c p \_r$ após $c p \_i n i$ no sentido anti-horário em $\mathbb{G}$;

6. $u \leftarrow$ Primeiro vértice da lista de adjacência de $c p \_r$ após $c p \_p$ no sentido horário em $\mathbb{G}$;

7. Enquanto $u \neq c p \_$ini faça

8. $\quad \mathbb{G}^{\prime} \leftarrow \mathbb{G}^{\prime}-\left\{\left(c p \_r, u\right)\right\}$

9. $\quad$ u.BordaDireita $\leftarrow$ Verdadeiro;

10. $u \leftarrow$ Primeiro vértice da lista de adjacência de $c p \_r$ após $u$ no sentido horário em $\mathbb{G}$;

11. $\quad c p_{-} \mathrm{NO} \leftarrow c p_{-} \mathrm{NE}$;

12. prox $\leftarrow$ Primeiro vértice da lista de adjacência de $c p \_i n i$ após $c p \_N E$ no sentido horário em $\mathbb{G}$;

13. Enquanto prox.BordaEsquerda $=1-$ BordaEsqAtual e 


$$
\text { prox.Pertence } G C D=\text { Falso faça }
$$

14. $\quad c p \_N O \leftarrow$ prox;

15. $\quad$ prox $\leftarrow$ Primeiro vértice da lista de adjacência de $c p \_i n i$ após prox no sentido horário em $\mathbb{G}$;

16. $\quad$ prox $\leftarrow c p_{-} N O$;

17. Repita

18. $u \leftarrow$ prox;

19. $\quad$ prox $\leftarrow$ Primeiro vértice da lista de adjacência de $c p \_i n i$ após $u$ no sentido anti-horário em $\mathbb{G}$;

20. Se $u=c p \_N E$ então

21.

u.ProxSuperior $\leftarrow N U L O$;

$22 . \quad$ Senão

23. u.ProxSuperior $\leftarrow$ Referência para prox;

24. u.BordaSuperior $\leftarrow$ Verdadeiro;

25. u.BordaEsquerda $\leftarrow 2$;

26. Até $\left(u=c p_{-} N E\right)$;

27. $\quad c p \_S O \leftarrow c p \_S E$;

28. $\operatorname{prox} \leftarrow$ Primeiro vértice da lista de adjacência de $c p \_p$ após $c p \_S E$ no sentido anti-horário em $\mathbb{G}$;

29. Enquanto prox.BordaEsquerda $=1-$ BordaEsqAtual

$$
\text { e prox.PertenceGCD = Falso faça }
$$

30. $\quad c p \_S O \leftarrow$ prox;

31. $\operatorname{prox} \leftarrow$ Primeiro vértice da lista de adjacência de $c p \_p$ após prox no sentido anti-horário em $\mathbb{G}$;

32. $\quad$ prox $\leftarrow c p \_S O$;

33. Repita

34. $u \leftarrow$ prox;

35. $\operatorname{prox} \leftarrow$ Primeiro vértice da lista de adjacência de $c p \_p$ após $u$ no sentido horário em $\mathbb{G}$;

36. Se $u=c p \_S E$ então

37. u.ProxInferior $\leftarrow N U L O$;

38. Senão

39. u.ProxInferior $\leftarrow$ Referência para prox;

40. u.BordaInferior $\leftarrow$ Verdadeiro;

41. $\quad$ u.BordaEsquerda $\leftarrow 2$;

42. Até $\left(u=c p \_S E\right)$;

43. $c p \_S O . P r o x E s q \leftarrow N U L O$;

44. Paths $\left(c p \_N O, c p \_N E, c p \_S O, c p \_S E, c p \_i n i, c p \_p, 1-\right.$ BordaEsqAtual $)$; 
Antes de apresentar uma análise da função, perceba que Processar-ComponentePendente utiliza o grafo $\mathbb{G}$.

As linhas 1-4 da função Processar-Componente-Pendente identificam o vértice $c p \_$ini. Percorremos a lista de adjacência do vértice $c p \_r$ no sentido horário, de modo que o vértice $c p \_i n i$ é o primeiro vértice pertencente ao atual caminho esquerdo da borda que é após $c p \_p$. Vamos identificar o primeiro vértice que foi encontrado após $c p \_p$ como $c p \_S E$. $\mathrm{O}$ vértice $c p \_S E$ pertence a componente pendente que queremos isolar. Ademais, temos que $c p \_S E \neq c p \_i n i$, pois caso contrário, a componente pendente seria vazia. Percorrendo a lista de adjacência do vértice $c p_{-} r$, identificamos o vértice $c p_{-} N E$, que não precisa ser diferente de $c p \_S E$ (linha 5). Assim, o caminho direito do subgrafo que será isolado está definido. Nas linhas 6-10 isolamos o parte direita da componente pendente do restante de $\mathbb{G}^{\prime}$.

Para identificar o vértice $c p \_N O$ percorremos a lista de adjacência do vértice $c p \_i n i$ no sentido horário a partir do vértice $c p \_N E$ (linhas 11-15). Basicamente, este passo consiste em percorrer os vértices do próximo caminho esquerdo da borda de $\mathbb{G}^{\prime}$ até encontrar um vértice que não foi adicionado a $\mathbb{D}$. Perceba que para qualquer vértice do segmento do atual caminho esquerdo que se inicia em $c p \_i n i$ e termina em $c p \_p$, temos que todos os vértices adjacentes a este devem pertencer ao próximo caminho esquerdo. Nas linhas 16-26, definimos o segmento que deverá ser o caminho superior da borda do subgrafo que está sendo isolado.

De modo semelhante a escolha de $c p_{-} N O$, podemos encontrar $c p \_S O$. As linhas 27-31 encontram $c p_{-} S O$ percorrendo a lista de adjacência do vértice $c p \_p$. Enquanto nas linhas 32-42 definimos o caminho inferior do subgrafo que será isolado.

Quando a linha 43 for executada, o subgrafo limitado pelos vértices $c p_{-} N O, c p_{-} N E$, $c p \_S O$ e $c p \_S E$ está isolado do resto de $\mathbb{G}^{\prime}$. Perceba que existem arestas que ligam os vértices dos caminhos superior, inferior e esquerdo da componente pendente aos vértices do atual caminho esquerdo da borda de $\mathbb{G}^{\prime}$, porém, como nosso algoritmo respeita os limites impostos por estes caminhos, podemos considerar que a componente pendente está isolada. Assim, na linha 43, ajustamos a variável do vértice $c p \_S O$ para que este seja o último vértice do caminho esquerdo da componente pendente.

Finalmente, na linha 45 executamos a recursão na componente pendente e todos os caminhos que cobrem os vértices desta, se iniciam no vértice $c p \_i n i$ e terminam no vértice $c p \_p$. Garantindo assim que não existe uma aresta que dê problema na definição do grafo dos caminhos dirigidos.

Para finalizar o algoritmo de construção do grafo dos caminhos dirigidos, precisamos definir os procedimentos Processar-Vértice-Borda-Direita e Processar-VérticeNÃO-BorDA-DiREITA que foram apresentados na função PATHS. 
No algoritmo PATHs, dependemos da posição do vértice $p$ para chamar a função Processar-VÉrtice-Borda-Direita ou a função Processar-VÉrtice-Não-BordaDiREITA, caso $p$ pertença ao caminho direito da borda iremos processá-lo utilizando Processar-VÉRTiCe-BordA-Direita, caso contrário, iremos processá-lo utilizando Processar-VÉRTICE-NÃO-BordA-DireitA.

A seguir, vamos apresentar a função Processar-VÉrtice-Borda-Direita. Basicamente, o algoritmo consiste nas seguintes tarefas:

Tarefa 1: Caso exista uma região não vazia acima de $p$ no grafo que está sendo processado, precisamos processar esta região. (Figura A.6A)

Tarefa 2: Caso estamos processando $p$ pela primeira vez e existe uma aresta $p t$, onde $t$ pertence ao atual caminho esquerdo da borda e $t$ não é adjacente a $p$ no atual caminho esquerdo da borda, então a região entre o caminho esquerdo da borda mais a aresta $p t$ deve ser processado. (Figura A.6B)

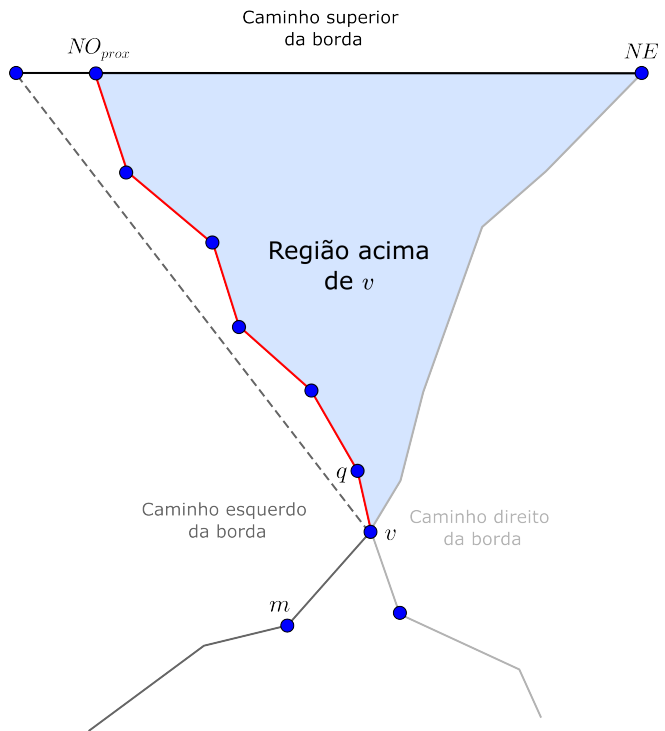

(A)

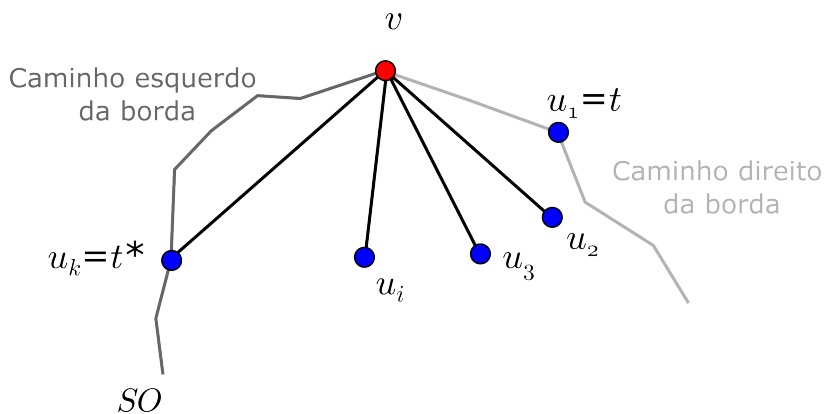

(B)

Figura A.6: Regiões que devem ser processadas por Processar-VÉRTICE-BordA-Direita. (A) Região acima de p. (B) Região entre o caminho esquerdo da borda e pt.[BS88]

$\mathrm{Na}$ figura A.6B, os vértices representados por $u_{i}$ representa o vértice da $i$-ésima iteração do laço das linhas 17-29 da função Processar-VÉrtice-Borda-Direita. 


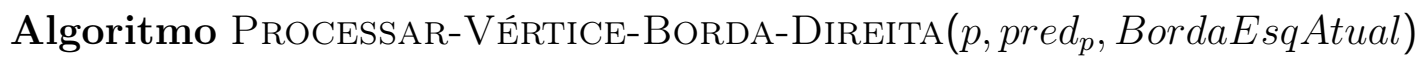

EnTRADA: Variáveis $p$, $\operatorname{pred}_{p}$,BordaEsqAtual.

SAÍDA: Grafos $\mathbb{D}$ e $\mathbb{G}^{\prime}$.

1. Se $\operatorname{pred}_{p} \neq N U L O$ e $\operatorname{pred}_{p}$.BordaDireita $=$ Falso então

2. $\quad$ q.ProxEsq $\leftarrow p$;

3. p.BordaEsquerda $\leftarrow 1-$ BordaEsqAtual;

4. $\quad m \leftarrow$ p.ProxEsq;

5. p.ProxEsq $\leftarrow N U L O$;

6. Paths $\left(N O_{\text {prox }}, N E, p, p\right.$, VerticeInicial, NULO, 1 - BordaEsqAtual);

7. $\quad$ p.ProxEsq $\leftarrow m$;

8. Se $p \neq S O$ então /* Caso contrário, não há nada para processar */

9. $\quad N O \leftarrow N E \leftarrow N O_{\text {prox }} \leftarrow q \leftarrow p$;

10. p.BordaEsquerda $\leftarrow 1-$ BordaEsqAtual;

11. VerticeInicial $\leftarrow p$;

12. Se ( $p$ está sendo visitado pela primeira vez) então

13. $\quad t \leftarrow$ p.ProxEsq;

14. Enquanto t.BordaDireita $=$ Falso então

15. $t \leftarrow$ Primeiro vértice da lista de adjacência de $p$ após $t$ no sentido anti-horário em $\mathbb{G}^{\prime}$;

16. $u \leftarrow t$

$17 . \quad$ Repita

18. Se $u$ BordaEsquerda $=$ BordaEsqAtual e $u \neq p$.ProxEsq então

19. Se u.PertenceGCD = Falso então

20. $\quad / * O$ vértice $u$ não foi adicionado a $\mathbb{D} * /$

21.

22. Processar-Corda ( $p, u$, BordaEsqAtual);

23.

24.

25.

\section{Senão}

/* O vértice $u$ é o vértice $S O$ */

Processar-Componente-Pendente $(u, p$, 1-BordaEsqAtual);

26.

27.

28.

29.

p.ProxEsq $\leftarrow u ;$

\section{Senão}

u.ChegouBordaDireita $\leftarrow p$;

$u \leftarrow$ Primeiro vértice da lista de adjacência de $p$ após $u$ no sentido horário em $\mathbb{G}^{\prime}$;

30. $\mathbb{G}^{\prime} \leftarrow \mathbb{G}^{\prime}-\{(p, p . \operatorname{Prox} E s q)\}$;

No momento que a função Processar-VÉrtice-Borda-Direita é chamada, todos os vértices predecessores de $p$ que estão no caminho esquerdo da borda já foram processados. 
Isso implica que todas as arestas que incidem nestes vértices em $\mathbb{G}$ não existem em $\mathbb{G}$. Caso $\operatorname{pred}_{p}=N U L O$, então $p=N O=N E$ então a região acima de $p$ é vazia. Caso $\operatorname{pred}_{p}$ pertence a borda direita então a região, caso exista, acima de $\operatorname{pred}_{p}$ já foi processada quando $\operatorname{pred}_{p}$ era o vértice $p$. Caso $\operatorname{pred}_{p}$ não é um vértice da borda direita, então existe ao menos um vértice que está a direita de $\operatorname{pred}_{p}$, logo, existe um vértice acima $p$ que está a sua direita. A condição da linha 1 identifica caso exista alguma região não vazia acima de $p$.

O segmento pontilhado do caminho esquerdo da borda da figura A.6A exibe qual parte do segmento será processado no procedimento. Quando este segmento for processado, iremos construir o próximo caminho da borda de modo que possua o segmento de $N O_{\text {prox }}$ até $q$. Para processar a região acima de $p$, completamos o caminho esquerdo da nova região (linhas 2-5) e chamamos a função PATHs na linha 6.

Quando a linha 8 for executada, a região acima de $p$ foi processada e deletada do grafo $\mathbb{G}^{\prime}$. Como $p$ é um vértice do caminho direito da borda, se $p$ for igual a $S O$ temos que $p=S E$. Assim, o processamento do grafo está completo. Ainda, teremos que Terminou é verdadeiro e p.ProxEsq = NULO. Então, o procedimento PATHS terminou.

Caso $p \neq S O$, então $p$ não é o último vértice do caminho esquerdo da borda. Este caso é ilustrado na figura A.6B. Se o vértice $p$ está sendo visitado pela primeira vez, então existe um único vértice $t$ que é vizinho de $p$ e pertence ao caminho direito da borda. Podemos enunciar um lema similar ao lema A.1 que garante que $t$ é único.

Lema A.3: Seja $\mathbb{G}^{\prime}$ um grafo que está sendo processado pelo algoritmo. $\mathbb{G}^{\prime}$ não contém uma aresta uv de modo que $u$ e $v$ pertençam ao caminho direito da borda e $u$ e $v$ não são adjacentes no caminho direito da borda.

Vamos percorrer a lista de adjacência de $p$ a partir de $t$ no sentido anti-horário. Seja $u$ um vértice qualquer desta travessia. Se $u$ pertence ao caminho esquerdo da borda, entretanto p.ProxEsq $\neq u$, temos que $u$ é o vértice $t^{*}$ ilustrado na figura A.6B. Assim, o caminho esquerdo da borda não pode ser processado normalmente, pois irá resultar que $p$ será um ancestral distante de $t^{*}$ em $\mathbb{D}$.

Considere o caso que $t^{*} \notin V(\mathbb{D})$, logo $p t^{*}$ é uma corda. Logo, devemos executar a função PROCESSAR-CORDA na região limitada por um segmento do caminho esquerdo da borda juntamente com a aresta $p t^{*}$. Após este processamento, toda esta região será removida de $\mathbb{G}^{\prime}$. Então a travessia da lista de adjacência de $p$ termina.

Agora, considere o caso que $t^{*}$ já foi adicionado a $\mathbb{D}$. Logo, temos que $t^{*}$ deve ser igual a $S O$, pois os vértices $N O$ e $S O$ são os únicos que podem sobrar após processar qualquer região. Assim, a região limitada pelo caminho esquerdo da borda e pela aresta $p t^{*}$ pode ser tratada pela função Processar-Componente-Pendente, onde $c p \_p=t^{*}$ e $c p \_r=p$. 
Depois do processamento da componente pendente, temos que pelo fato da componente ser excluída de $\mathbb{G}^{\prime}$, o vértice $t$ irá ser o próximo vértice adjacente a $p$ no sentido anti-horário. Deste modo, a travessia da lista de adjacência de $p$ está completa.

Caso $u$ não satisfaça as condições da linha 18, então u.ChegouBordaDireita deve ser alterado para uma referência de $p$ de modo que indique que $u$ é atingido por um vértice da borda direita $(p)$ utilizando somente uma única aresta. Esta variável será utilizada na função Processar-VÉrTice-NÃo-Borda-Direita para detectar uma corda. Setando esta variável agora, garantimos que não precisaremos percorrer a lista de adjacência de $p$ mais de uma vez, o que garante que o algoritmo não possua complexidade $O\left(n^{2}\right)$.

Finalmente, vamos apresentar a última rotina do algoritmo da construção do grafo de caminho dirigidos. Esta é o segundo caso do processamento processamento do vértice $p$ da função Paths. O algoritmo Processar-Vértice-NÃo-BordA-Direita trata quando o vértice não pertence ao caminho direito da borda. Ainda, este algoritmo deve, além de processar o vértice, construir parte do próximo caminho esquerdo da borda. Inicialmente, o segmento do próximo esquerdo da borda se inicia no vértice $N O_{\text {prox }}$ e termina no vértice $q$, onde $p$ é adjacente a $q$.

Neste algoritmo, vamos percorrer a lista de adjacência do vértice $p$ no sentido horário a partir do vértice $q$ (linhas 3-34). Seja $r$ o vértice que está sendo examinado em uma iteração deste laço. O caso tratado nas linhas 6-12 é análogo ao tratado nas linhas (19-25) do algoritmo Processar-VÉRTiCe-BordA-Direita. As linhas 14-26 tratam o caso em que $r$ não foi visitado anteriormente. Nas linhas 15-18 adicionamos $r$ ao próximo caminho esquerdo da borda. Os casos especiais das regiões críticas que podem ser criadas pela aresta pr são detectados e tratados nas linhas 19-26.

As linhas 27-29 identificam e tratam o último caso para $r$, o da componente pendente. Neste caso, o vértice $r$ será processado por dois vértices não adjacentes, $p$ e um vértice do próximo caminho esquerdo da borda.

Nas linhas 30-34, adicionamos o vértice $r$ ao próximo caminho esquerdo da borda. Executamos nestas linhas o mesmo processamento da Tarefa 2 do algoritmo PROCESSARVÉRTICE-BordA-Direita. Finalmente, a linha 32 processa o subgrafo definido pelo próximo caminho esquerdo da borda.

Um último detalhe que deve ser explicado é o por que a variável ChegouBordaDireita foi utilizada de modo a melhorar a complexidade computacional. Note que por causa do algoritmo Processar-VÉrtice-Borda-Direita, se as condições da linha 31 são verdadeiras, temos que o vértice $r$ não foi adicionado a $\mathbb{D}$. Entretanto, podemos executar o algoritmo Processar-VÉRTICE-BORDA-DiREITA para o vértice $p$ mais do que uma única vez e se percorrermos mais de uma única vez a lista de adjacência de $p$ nosso algoritmo não será executado em tempo polinomial. 
Vamos voltar um pouco e analisar novamente o algoritmo PROCESSAR-VÉRTICE-BORDADiREITA. Se não estamos visitando $p$ pela primeira vez, todos os vértices adjacentes a $p$ que satisfazem as condições da linha 18, continuarão satisfazendo e os grafos que devem ser processados já foram processados, ou seja, o processamento das linhas 19-25 não será útil. Ainda, para o vértice $r$, este caso foi tratado nas linhas 6-12 do algoritmo Processar-VÉrtice-NÃo-Borda-Direita. Assim, a lista de adjacência do vértice $p$ que será percorrida na função PROCESSAR-VÉRTICE-BORDA-DiREITA só deverá executar a linha 27. Entretanto, temos que u.ChegouBordaDireita já possui uma referência para o vértice p. Logo, a execução das linhas 12-29 são necessárias somente quando estamos visitando $p$ pela primeira vez.

Algoritmo Processar-VÉRtice-Não-Borda-Direita $(p, q$, BordaEsqAtual)

ENTRADA: Vértices $p, q$ e variável BordaEsqAtual.

SAÍDA: Grafos $\mathbb{D}$ e $\mathbb{G}^{\prime}$.

1. $\mathbb{G}^{\prime} \leftarrow \mathbb{G}^{\prime}-\{p q\}$;

2. Sair $\leftarrow$ Falso;

3. Enquanto (Existe um vértice adjacente a $p$ ) e Sair $=$ Falso faça

4. $\quad r \leftarrow$ Primeiro vértice da lista de adjacência de $p$ após $q$ no sentido horário em $\mathbb{G}^{\prime}$;

5. Se r.BordaEsquerda $=$ BordaEsqAtual e $r \neq p$.ProxEsq então

6. Se r.Pertence $G C D=$ Falso então

7. $\quad / *$ vértice $r$ não foi adicionado a $\mathbb{D} * /$

8. Processar-Corda ( $p, r$, BordaEsqAtual);

9. Senão

10. $\quad$ * O vértice $r$ é o vértice $S O$ */

11. Processar-Componente-Pendente( $r, p, 1$-BordaEsqAtual);

12. p.ProxEsq $\leftarrow r$;

13. Senão Se r.BordaEsquerda $=2$ então

14. $\quad / * r$ é um vértice novo */

15. $\quad$ q.ProxEsq $\leftarrow r$;

16. r.BordaEsquerda $=1-$ BordaEsqAtual;

17. $q \leftarrow r$;

18. $\quad$ Terminou $\leftarrow$ Falso;

19. Se r.BordaSuperior $=$ Verdadeiro então

20. Processar-CANTO-Superior-Esquerdo $(p, r)$;

21. $N O_{\text {prox }} \leftarrow r$;

22. Senão Se $r$.BordaInferior $=$ Verdadeiro e $p \neq S O$ então

23. Processar-CANTO-Inferior-Esquerdo $(p, r)$;

24. $S O_{\text {prox }} \leftarrow r$; 
25. p.ProxEsq $\leftarrow N U L O$;

26.

Sair $\leftarrow$ Verdadeiro;

27. Senão Se r.BordaEsquerda $=1-$ BordaEsqAtual então

28. $q \leftarrow r$

29. Processar-Componente-Pendente ( $p, r$, BordaEsqAtual);

30. $\mathbb{G}^{\prime} \leftarrow \mathbb{G}^{\prime}-\{p r\}$;

31. Se $r . B o r d a D i r e i t a R e c h e a d=N O$ e $N O \cdot \operatorname{ProxE} s q \neq r$ então

32. Processar-Corda (NO, r, 1 - BordaEsqAtual);

33. NO.ProxEsq $\leftarrow r$;

34. $q \leftarrow r$ 


\section{REFERÊNCIAS BIBLIOGRÁFICAS}

[BM08] J. A. Bondy and U. S. R. Murty, Graph theory, Graduate Texts in Mathematics, vol. 244, Springer, New York, 2008. MR 2368647 (2009c:05001) 106

[BS85] Jayaram Bhasker and Sartaj Sahni, A linear algorithm to find a rectangular dual of a planar graph, Technical report tr 85-26, Computer Science Dept., University of Minnesota, Minneapolis, 1985. 18

[BS87] _ A linear time algorithm to check for the existence of a rectangular dual of a planar triangulated graph, Networks 17 (1987), no. 3, 307-317. 18, 47

[BS88] _ A linear algorithm to find a rectangular dual of a planar triangulated graph, Algorithmica 3 (1988), no. 2, 247-278. 18, 47, 52, 53, 54, 57, 58, 61, 62, 65, $67,70,173,174,177,180,183$

[Fra05] András Frank, On Kuhn's Hungarian method - a tribute from Hungary, Naval Res. Logist. 52 (2005), no. 1, 2-5. 104

[He93] Xin He, On finding the rectangular duals of planar triangular graphs, SIAM J. Comput. 22 (1993), no. 6, 1218-1226. 18, 73, 117

[HK73] John E. Hopcroft and Richard M. Karp, An $n^{5 / 2}$ algorithm for maximum matchings in bipartite graphs, SIAM J. Comput. 2 (1973), 225-231. 104

[KH97] Goos Kant and Xin He, Regular edge labeling of 4-connected plane graphs and its applications in graph drawing problems, Theoret. Comput. Sci. 172 (1997), no. 1-2, 175-193. MR 1432861 (98b:68152) 18

[KK84] Krzysztof Koźmiński and Edwin Kinnen, An algorithm for finding a rectangular dual of a planar graph for use in area planning for VLSI integrated circuits, Proceedings of the 21st Design Automation Conference, june 1984, pp. 655 - 656. $18,21,43,45,46$

[KK85] _ Rectangular duals of planar graphs, Networks 15 (1985), no. 2, 145-157. $18,21,27,32,35,36,38$ 
[KR74] D. Kelly and I. Rival, Planar lattices, Notices of the american mathematical society 21 (1974), no. 1, A46 (English). 79

[Kur30] K. Kuratowski, Sur le problème des courbes gauches en topologie, Fund. Math 15 (1930), no. 271-283, 79. 9

[LL90] Yen Tai Lai and Sany M. Leinwand, A theory of rectangular dual graphs, Algorithmica 5 (1990), no. 4, 467-483. 18, 93, 97, 104, 106, 107

[Men27] Karl Menger, Zur allgemeinen kurventheorie, Fund. Math 10 (1927), no. 5, 95-115. 22

[PT88] F.P. Preparata and R. Tamassia, Fully dynamic techniques for point location and transitive closure in planar structures, Foundations of Computer Science, 1988., 29th Annual Symposium on, oct 1988, pp. 558 -567. 79

[RNG04] Md. Saidur Rahman, Takao Nishizeki, and Shubhashis Ghosh, Rectangular drawings of planar graphs, J. Algorithms 50 (2004), no. 1, 62-78. 117

[RNN98] Md. Saidur Rahman, Shin-ichi Nakano, and Takao Nishizeki, Rectangular grid drawings of plane graphs, Comput. Geom. 10 (1998), no. 3, 203-220. MR 1625496 (99c:05055) 117, 129, 133, 134, 135, 137, 139, 140, 148, 149, 151, 152, 153, 154, 155,158

[RT86] Pierre Rosenstiehl and Robert E. Tarjan, Rectilinear planar layouts and bipolar orientations of planar graphs, Discrete Comput. Geom. 1 (1986), no. 4, 343-353. 80

[Tam87] Roberto Tamassia, On embedding a graph in the grid with the minimum number of bends, SIAM J. Comput. 16 (1987), no. 3, 421-444. MR 889400 (88h:68086) 152

[Tho84] C. Thomassen, Plane representations of graphs, Progress in graph theory (Waterloo, Ont., 1982), Academic Press, Toronto, ON, 1984, pp. 43-69. MR 776790 (86g:05032) 117, 119, 129, 143

[TT89] Roberto Tamassia and Ioannis G. Tollis, Planar grid embedding in linear time, IEEE Trans. Circuits and Systems 36 (1989), no. 9, 1230-1234. MR 1009276 (90g:05074) 152

[TV89] R. Tamassia and J. S. Vitter, Optimal parallel algorithms for transitive closure and point location in planar structures, Proceedings of the first annual ACM symposium on Parallel algorithms and architectures (New York, NY, USA), SPAA '89, ACM, 1989, pp. 399-408. 79 